\title{
Imidazol-5-one as an Acceptor in Donor-Acceptor Cyclopropanes: Cycloaddition with Aldehydes
}

\author{
Andrey A. Mikhaylov, ${ }^{*, a}$ Andrei V. Kuleshov, ${ }^{\text {a,b }}$ Pavel N. Solyev, ${ }^{c}$ Alexander A. Korlyukov, ${ }^{\text {d }}$ \\ Pavel V. Dorovatovskii, ${ }^{\mathrm{e}}$ Konstantin S. Mineev ${ }^{\mathrm{a}}$ and Mikhail S. Baranov*,a,f \\ a Shemyakin-Ovchinnikov Institute of Bioorganic Chemistry of the Russian Academy of Sciences, 16/10 Miklukho- \\ Maklaya St., Moscow, 117997, Russia; \\ b Chemistry Department, Moscow State University, 1-3 Leninskiye Gory, Moscow, 119991, Russia; \\ ${ }^{c}$ Engelhardt Institute of Molecular Biology of the Russian Academy of Sciences, 32 Vavilova St., Moscow, 119991, \\ Russia; \\ ${ }^{\mathrm{d}}$ Nesmeyanov Institute of Organoelement Compounds, 28 Vavilova St., Moscow, 119334, Russia; \\ e National Research Center "Kurchatov Institute", 1, Akademika Kurchatova P1., Moscow, 123182, Russia \\ ${ }^{\mathrm{f}}$ Pirogov Russian National Research Medical University, 1 Ostrovitianov St., Moscow, 117997, Russia.
}

\section{Table of contents}

Table of contents S1

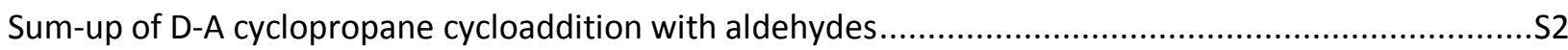

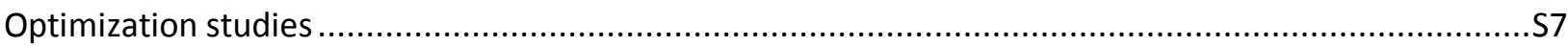

Competitive reactions with usual D-A cyclopropane ...............................................................

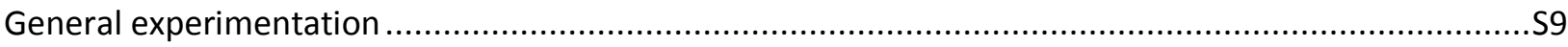

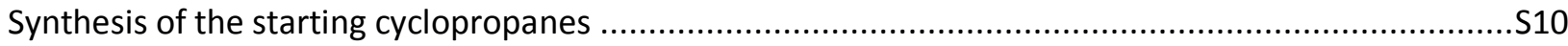

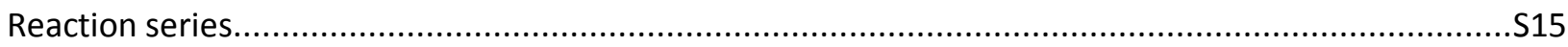

Gram-scale experiment \& Further examples of stereoselective cycloaddition .................................S38

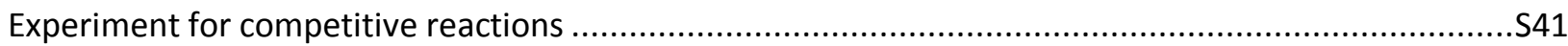

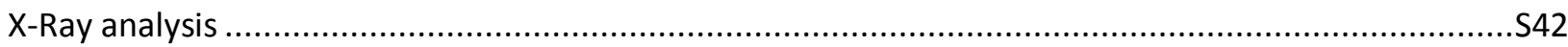

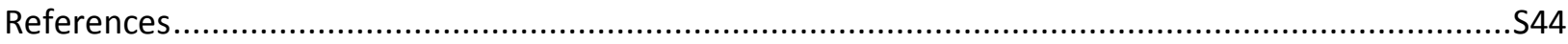

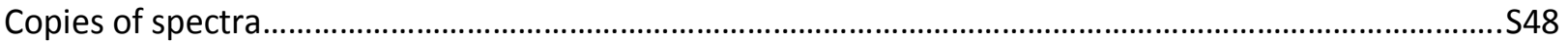


Sum-up of D-A cyclopropane cycloaddition with aldehydes

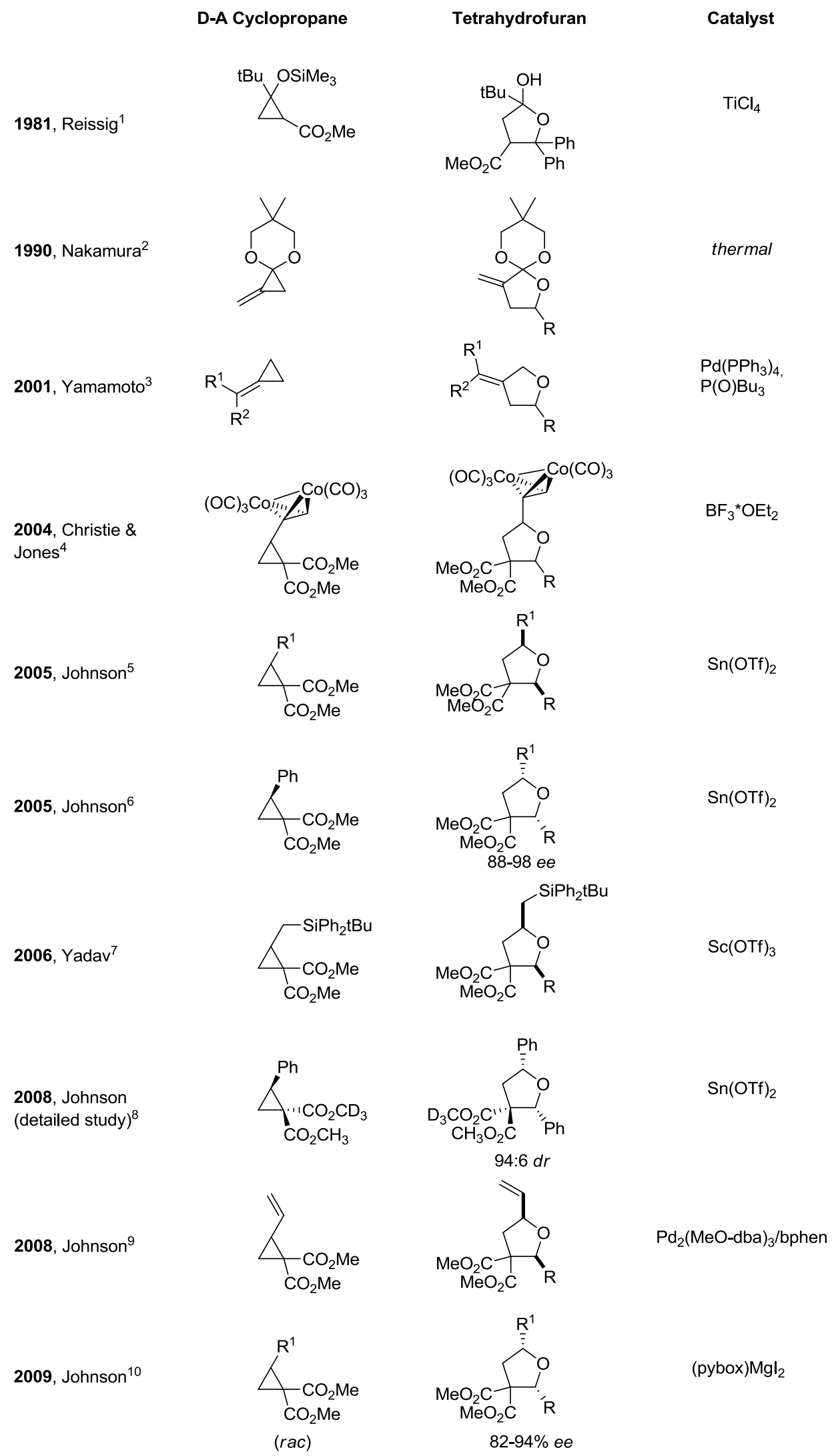

Scheme SI1. D-A cyclopropanes cycloadditions to form tetrahydrofurans. 
2009, Christie ${ }^{11}$

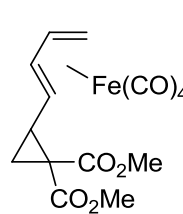

2010, Wang ${ }^{12}$

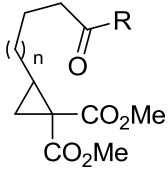

2011, Johnson ${ }^{13}$

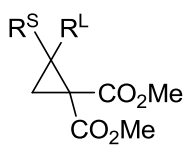

2011, Yang ${ }^{14}$

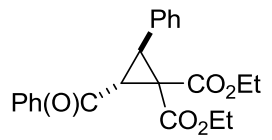

2011, Yorimitsu \& Oshima ${ }^{15}$<smiles>CCCCCC(CCCC)=C1CC1(OC)C(C)=O</smiles>

2011, Zhang ${ }^{16}$<smiles>CCOC1[C@H]2[C@H]([C@H]3COC(=O)[C@H]3OCC)[C@@H]12</smiles>

2012, Waser ${ }^{17}$

2012, Waser ${ }^{18}$

2012, Wang ${ }^{19}$<smiles>CCOC(=O)C1(C(=O)OCC)CC1[NH2+]</smiles><smiles>CCOC(=O)C1(C(=O)OCC)CC1[NH2+]c1ccccc1</smiles>
$(S)$ - or $(R)$ -<smiles>[R]C1OC(/C=C/C=C)CC1(COC)C(C)(C)C(=O)OC(=O)C(F)(F)F</smiles>

$\mathrm{ZnBr}_{2}$<smiles>[R]C12CCC(O1)C1CCC2(COC)O1</smiles>

$\mathrm{Sc}(\mathrm{OTf})_{3}$ $\mathrm{Sn}(\mathrm{OTf})_{2}$ $\mathrm{AlCl}_{3}$

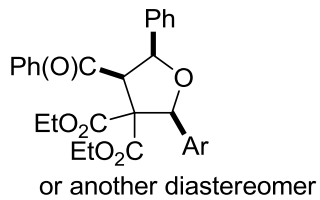<smiles>CCCCC1=C(C(=O)OC)C(CCOC)OC1</smiles>

$\mathrm{Yb}(\mathrm{OTf})_{3}$ $\mathrm{Sc}(\mathrm{OTf})_{3}$<smiles></smiles><smiles>[R]C1OC([NH2+]c2ccccc2)CC1(CC)OCC</smiles>
$\mathrm{FeCl}_{3}-\mathrm{Al}_{2} \mathrm{O}_{3}$<smiles>[R]C1([R3])OC([NH2+]c2ccccc2)CC1(C)OCC</smiles>

$\mathrm{SnCl}_{4}$<smiles>[R]C#C[C@]1(C#[R]O[R])C[C@@H]2CC[C@@H]1O2</smiles>

$\mathrm{Ni}\left(\mathrm{ClO}_{4}\right)_{2}{ }^{*} 6 \mathrm{H}_{2} \mathrm{O}$

Scheme SI1 (continued). D-A cyclopropanes cycloadditions to form tetrahydrofurans. 


\section{D-A Cyclopropane}

2013, Reisman ${ }^{20}$<smiles>CC1(C)C2(C)CCCC(=O)C12C#N</smiles>

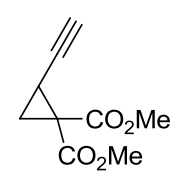

2013, Sakata \& Nishibayashi ${ }^{21}$

2013, Niggemann 22

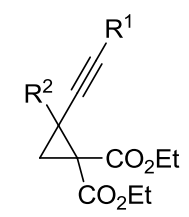

2013, Zhou ${ }^{23}$<smiles>CC(C)(C)N1C(=O)[C@@]2(C[C@H]2c2ccccc2)c2ccccc21</smiles>

2013, Liu $^{24}$

2013, Shao 25

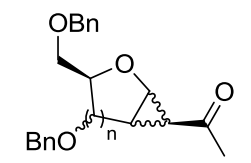

2014, Waser ${ }^{26}$

2014, Waser ${ }^{27}$

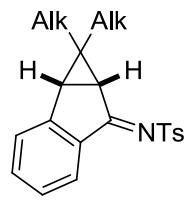
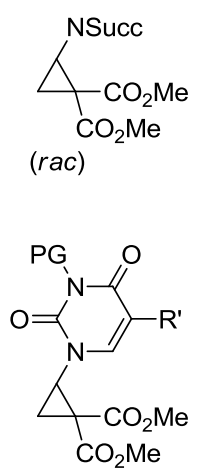

2014, Shi \& $\mathrm{Cao}^{28}$

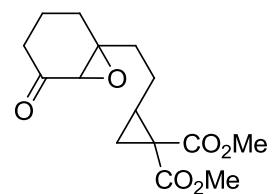

$\mathrm{Sc}(\mathrm{OTf})_{3}$

$\left[\mathrm{Cp}{ }^{\star} \mathrm{Ru}(\mathrm{SMe}) \mathrm{Cl}\right]_{2}$ $\mathrm{Ca}(\mathrm{NTf})_{2} / \mathrm{Bu}_{4} \mathrm{NPF}_{6}$ $\mathrm{Cu}(\mathrm{OTf})_{2}$ $\mathrm{AgNTf}_{2}$

$\mathrm{InCl}_{3}$<smiles>[R8]OCC1OC2OC([R])[C@H](C(C)=O)[C@@H]2[C@H]1O</smiles>

$\mathrm{Cu}\left(\mathrm{ClO}_{4}\right)_{2} /$ chiral Box

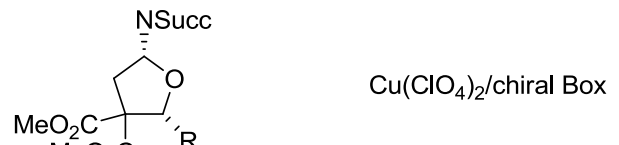

$\ln (\mathrm{OTf})_{3}$ or $\mathrm{SnCl}_{4}$

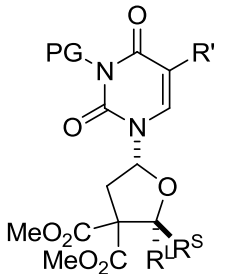

$\mathrm{BF}_{3}{ }^{*} \mathrm{OEt}_{2}$

Scheme SI1 (continued). D-A cyclopropanes cycloadditions to form tetrahydrofurans 
2014, Kurahashi \& Matsubara $^{29}$

2014, Wang ${ }^{30}$

2015, Wang ${ }^{31}$

2015, Wang ${ }^{32}$
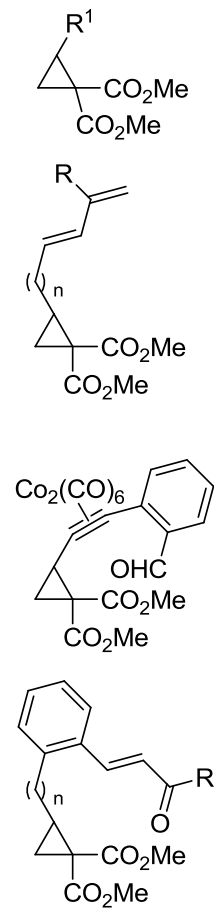

2015, Maulide ${ }^{33}$

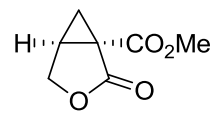

2016, Maulide ${ }^{34}$

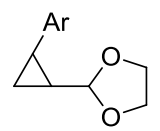

2016. Wang ${ }^{35}$

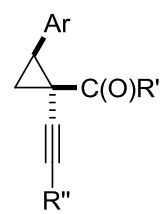

2016, Xiang \& Yang $^{36}$<smiles>COC(=O)C1(C(=O)Nc2ccccc2C=O)CC1c1ccccc1</smiles><smiles>O=C(O)C1CC1Br</smiles>

2017, Rawat $^{37}$<smiles>[R]C1CC(C(=O)OC)(C(C)OC)C([R])O1</smiles>

[Co(TPP)]TFPB<smiles>[R]C1=C2N[C@@H]3CC(COC)(C(=O)OC)C2([R])C3([R])CCC1</smiles>

reflux, then $\mathrm{Sc}(\mathrm{OTf})_{3}$

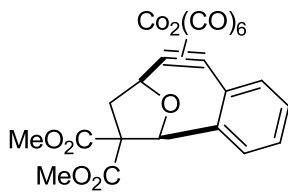

$\mathrm{Sc}(\mathrm{OTf})_{3}$<smiles>[R]C1(C(=O)OC)CC2[CH]c3ccccc3C=CC21[R]</smiles>

$\mathrm{hv}, \mathrm{Sc}(\mathrm{OTf})_{3}$

$\mathrm{Sc}(\mathrm{OTf})_{3}$<smiles>COC[C@H]1OC[C@@H]2COC(=O)[C@@]12COC</smiles>

TBSOTf<smiles>[R]C1O[C@@H]([Al])C[C@@H]1C1OCCO1</smiles>

$\mathrm{Sc}(\mathrm{OTf})_{3}$<smiles>[R]C#C[C@]1(C#[R]O[Na])CC(Br)OC1[Al]</smiles>

$\mathrm{TiCl}_{4}$<smiles>COC(=O)[C@]12C[C@H](c3ccccc3)O[C@H]1c1ccccc1NC2=O</smiles>

$\mathrm{BF}_{3}{ }^{*} \mathrm{OEt}_{2}$<smiles></smiles>

Scheme SI1 (continued). D-A cyclopropanes cycloadditions to form tetrahydrofurans 
2017, Yan \& Zhou ${ }^{38}$

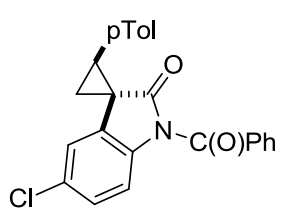<smiles>O=C1Nc2ccc(Cl)cc2[C@]12CC([18O])O[C@H]2CCl</smiles>

$\mathrm{Sc}(\mathrm{OTf})_{3}$

2017, Zhu \& Zhou ${ }^{39}$<smiles>[N-]c1ccccc1[C@]12COC(=O)[C@H]1[C@H]2c1ccccc1</smiles><smiles>[NH3+]c1ccccc1[C@]12COC(=O)[C@@]1(S)[C@@H](c1ccccc1)O[C@H]2c1ccccc1</smiles>
$\mathrm{Sc}(\mathrm{OTf})_{3}$

2017, $\mathrm{Ma}^{40}$

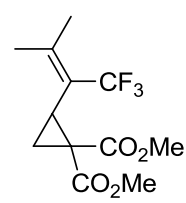<smiles>COC(=O)C1(C(C)=O)C[C@@H](C(=C(C)C)C(F)(F)F)O[C@H]1c1ccccc1</smiles>
$\mathrm{Sc}(\mathrm{OTf})_{3}$

2018, Werz ${ }^{41}$

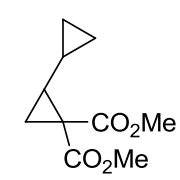<smiles>[R]C1OC(C2CC2)CC1(C(C)=O)C(=O)OC</smiles>

$\mathrm{Sn}(\mathrm{OTf})_{2}$

2018, Ruijter ${ }^{42}$<smiles>[R15]N1C[C@]2(C)[C@H](C=C)[C@]2(C(C)=O)C1=O</smiles><smiles>[Y16]N1CC[C@]2(C)C(C=C)OC(c3ccccc3)[C@@]12C(C)=O</smiles>
$\mathrm{Sn}(\mathrm{OTf})_{2}$

2018, Yang ${ }^{43}$<smiles>COC(=O)[C@]12C(=O)OC[C@H]1[C@@H]2Br</smiles><smiles>COC(=O)[C@]12C(=O)OC[C@H]1[C@@H](Br)O[C@H]2Br</smiles>
$\mathrm{Sc}(\mathrm{OTf})_{3}$ 2018, Yang ${ }^{44}$<smiles>COC(=O)C1C([Al])OC(=O)C1OC</smiles>

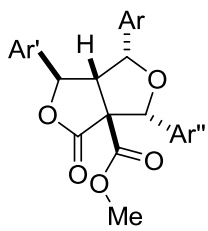
$\mathrm{Sn}(\mathrm{OTf})_{2}$

2018, Yang ${ }^{45}$<smiles>COC(=O)C1(C(C)=O)C(Br)[C@H]1C(=O)N1CCCC1=O</smiles><smiles>COC(=O)C1(C(=O)OC)[C@H]([Al])O[C@H]([Al])[C@H]1C(=O)N1CCCC1=O</smiles>

$\mathrm{AlCl}_{3}$

Scheme SI1 (continued). D-A cyclopropanes cycloadditions to form tetrahydrofurans 


\section{Optimization studies}<smiles>COc1ccc(C2CC23CC3)cc1</smiles>

(1 equiv.)

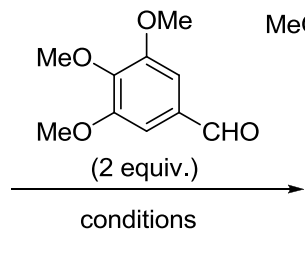<smiles>COc1ccc(C2C[C@@]3(N=C(C)N(C)C3=O)C(c3cc(OC)c(OC)c(OC)c3)O2)cc1</smiles><smiles>COc1cc(C2N[C@@]3(CC(c4ccc(F)cc4)O3)C(=O)N2C)cc(OC)c1OC</smiles>

Table SI1. Optimisation of the reaction conditions

\begin{tabular}{|c|c|c|c|c|c|c|c|}
\hline \multirow{2}{*}{ Entry } & \multirow{2}{*}{ Promoter } & \multirow{2}{*}{ Conditions } & \multirow{2}{*}{ Conversion $^{a}$} & \multicolumn{3}{|c|}{ Yield, \% } & \multirow{2}{*}{$d r^{\mathrm{a}}$} \\
\hline & & & & Combined $^{\mathrm{a}}$ & $\mathbf{1}^{\mathrm{b}}$ & $1^{\mathrm{b}}$ & \\
\hline 1 & $\begin{array}{c}\text { Lewis acids: } \\
\mathrm{SnCl}_{4}(0.5 \text { equiv. }) \\
\mathrm{Sc}(\mathrm{OTf})_{3}(0.2 \text { equiv }) \\
\mathrm{Sn}(\mathrm{OTf})_{2}(0.1 \text { equiv })\end{array}$ & $\mathrm{CH}_{2} \mathrm{Cl}_{2}(0.1 \mathrm{M})$, r.t., $4 \mathrm{~h}$ & $<5 \%$ & $\mathrm{nd}^{\mathrm{c}}$ & nd & nd & $\mathrm{Nd}$ \\
\hline 2 & TFA (1.1 equiv.) & $\mathrm{CH}_{2} \mathrm{Cl}_{2}(0.1 \mathrm{M})$, r.t., $4 \mathrm{~h}$ & $<5 \%$ & nd & nd & nd & $\mathrm{Nd}$ \\
\hline 3 & $\mathrm{TsOH}^{*} \mathrm{H}_{2} \mathrm{O}$ (1.1 equiv.) & $\mathrm{CH}_{2} \mathrm{Cl}_{2}(0.1 \mathrm{M})$, r.t., $\mathrm{lh}$ & $100 \%$ & 89 & 47 & 42 & $1: 1.1$ \\
\hline 4 & $\mathrm{MsOH}(1.1$ equiv.) & $\mathrm{CH}_{2} \mathrm{Cl}_{2}(0.1 \mathrm{M})$, r.t., $1 \mathrm{~h}$ & $100 \%$ & ca. 40 & nd & nd & $1.1: 1$ \\
\hline 5 & CSA (1.1 equiv.) & $\mathrm{CH}_{2} \mathrm{Cl}_{2}(0.1 \mathrm{M})$, r.t., $1 \mathrm{~h}$ & ca. $90 \%$ & ca. 70 & nd & nd & $1.3: 1$ \\
\hline 6 & $\mathrm{PhSO}_{3} \mathrm{H}$ (1.1 equiv.) & $\mathrm{CH}_{2} \mathrm{Cl}_{2}(0.1 \mathrm{M})$, r.t., $\mathrm{lh}$ & $100 \%$ & 93 & 49 & 44 & $1.1: 1$ \\
\hline 7 & TfOH (1.1 equiv.) & $\mathrm{CH}_{2} \mathrm{Cl}_{2}(0.1 \mathrm{M})$, r.t., $30 \mathrm{~min}$ & $100 \%$ & ca. $70^{\mathrm{d}}$ & nd & nd & $1.3: 1^{\mathrm{d}}$ \\
\hline 8 & TsOH (1.1 equiv.) & $\mathrm{CH}_{2} \mathrm{Cl}_{2}(0.1 \mathrm{M})$, r.t., $30 \mathrm{~min}$ & $100 \%$ & 85 & 46 & 39 & $1.2: 1$ \\
\hline 9 & TsOH ( 0.1 equiv. $)$ & $\mathrm{CH}_{2} \mathrm{Cl}_{2}(0.1 \mathrm{M})$, r.t., $1 \mathrm{~h}$ & ca. $15 \%$ & ca. 15 & nd & nd & $\mathrm{Nd}$ \\
\hline 10 & TsOH (1.1 equiv.) & toluene $(0.1 \mathrm{M})$, r.t., $2 \mathrm{~h}$ & $100 \%$ & 78 & nd & nd & $1.3: 1$ \\
\hline $11^{e}$ & Ts $O H$ (1.1 equiv.) & $\mathrm{CH}_{2} \mathrm{Cl}_{2}(0.1 \mathrm{M}), 0^{\circ} \mathrm{C}, 3 \mathrm{~h}$ & $100 \%$ & 91 & 51 & 40 & 1.3:1 \\
\hline
\end{tabular}

a - determined by NMR with internal standard; ${ }^{b}$ - isolated yield; ${ }^{c}$ - not determined; ${ }^{d}$ - reproducibility issiues observed; ${ }^{\mathrm{e}}-3$ equiv. of aldehyde was used 
Competitive reactions with usual D-A cyclopropane

(a)
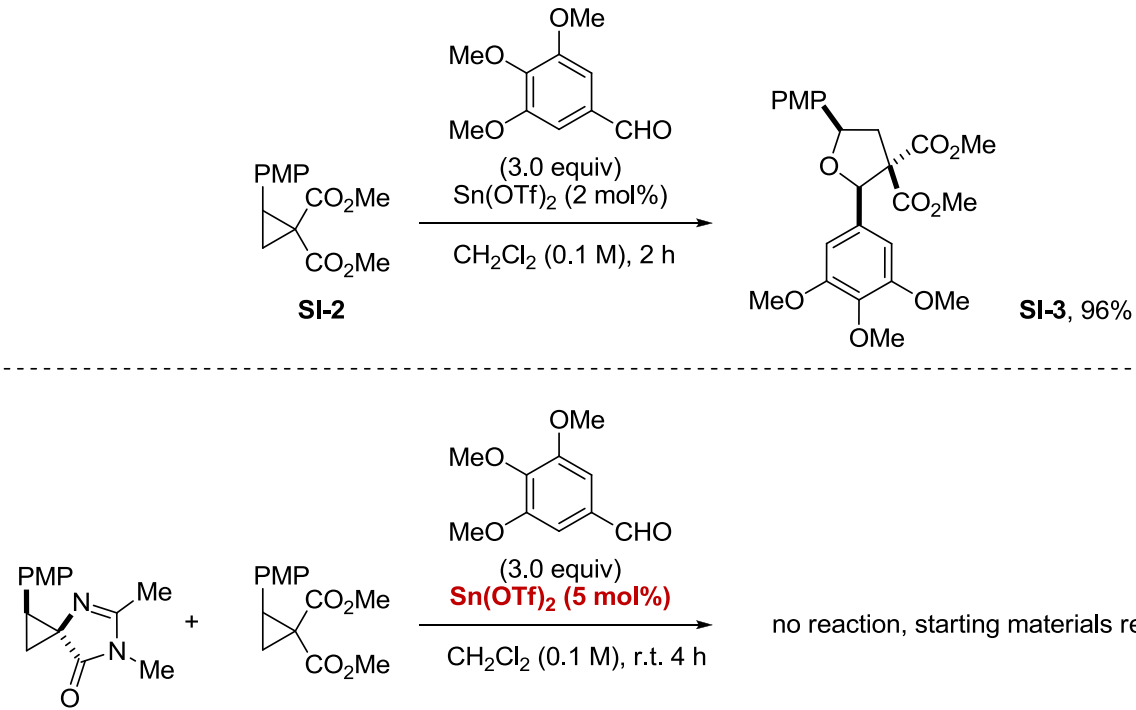

1a (1 equiv.) SI-2 (1 equiv.)
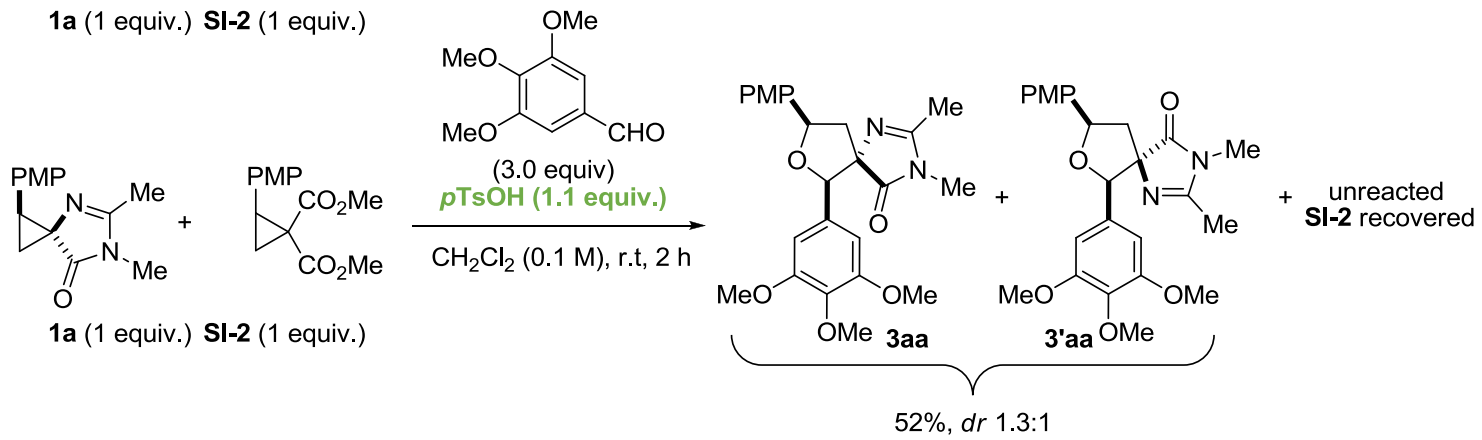

Scheme SI2. a) Production of cycloadduct under Lewis acid catysis with common D-A cyclopropane (1,1cyclopropane dicarboxylate). b) Competition experiments with common D-A cyclopropane (1,1cyclopropane dicarboxylate) under Lewis acid and TsOH activation. 


\section{General experimentation}

All reactions were performed in round-bottom flasks fitted with rubber septa. Reactions sensitive to air and/or moisture were performed under a positive pressure of argon. Air- and moisture-sensitive liquids were transferred by syringe. Analytical thin-layer chromatography (TLC) was performed using aluminum plates pre-coated with silica gel (silica gel 60 F254, Merck). TLC plates were visualized by exposure to $254 \mathrm{~nm}$ ultraviolet light (UV) or were stained by submersion in acidic ethanolic solution of vanillin followed by brief heating (vanillin) or submersion in aqueous potassium permanganate solution followed by extensive washing with water $\left(\mathrm{KMnO}_{4}\right)$. Flash-column chromatography was carried out on silica gel (60 $\AA$, 230-400 mesh, Sigma-Aldrich). All solvents for chromatography and extractions were technical grade and distilled prior use.

All reagents were obtained from commercial suppliers (Sigma-Aldrich, Acros Organics, Fluorochem, $\mathrm{ABCR}$ ) and were used without further purification. DMSO was dried over activated molecular sieves, decanted and distilled in vacuo. $\mathrm{CH}_{2} \mathrm{Cl}_{2}$ was dried by distillation over $\mathrm{CaH}_{2}$ prior use.

Nuclear magnetic resonance spectra were recorded using Bruker Furier 300, Bruker Avance II 700 instruments at indicated temperature. Data are represented as follows: chemical shift, multiplicity ( $\mathrm{s}=$ singlet, $\mathrm{d}=$ doublet, $\mathrm{t}=$ triplet, $\mathrm{q}=$ quartet, $\mathrm{m}=$ multiplet and/or multiple resonances), coupling constant $(J)$ in Hertz, integration. Proton chemical shifts are expressed in parts per million (ppm, $\delta$ scale) and are referenced to residual protium in the NMR solvents $\left(\mathrm{CHCl}_{3}, \delta 7.26 \mathrm{ppm} ; \mathrm{CHD}_{2} \mathrm{SOCD}_{3}, \delta 2.50 \mathrm{ppm}\right.$,). Carbon chemical shifts are expressed in parts per million (ppm, $\delta$ scale) and are referenced to the carbon resonances of the NMR solvents $\left(\mathrm{CDCl}_{3}, \delta 77.16 \mathrm{ppm} ;\left(\mathrm{CD}_{3}\right)_{2} \mathrm{SO}, \delta 39.52 \mathrm{ppm}\right)$.

High-resolution mass spectra were recorded on a Bruker micrOTOF-Q II mass spectrometer using electrospray ionization (ESI-TOF). Melting points were determined on Kofler melting point apparatus and are uncorrected.

Unhydrous $p$-toluenesulfonic acid was prepared by drying commercial hydrate at $90{ }^{\circ} \mathrm{C}$ in high vacuo ( $\mathrm{p}<0.1$ torr) for 12 hours. Arylidene imidazolones were obtained according to the appropriate literature procedures. ${ }^{46,47}$ The following compounds were obtained according to literature procedures: ethyl 2-((1methoxyethylidene)amino)acetate, ${ }^{48}$ 4-(difluoromethoxy)-3-methoxybenzaldehyde, ${ }^{49}$ cyclopropane SI-2. ${ }^{50}$ 4-Nitro-3-methoxybenzaldehyde was obtained by nitration of 3-methoxybenzaldehyde according to literature procedure but isolated by column chromatography. ${ }^{51}$ 


\section{Synthesis of the starting cyclopropanes}

General procedure: Sodium hydride $(0.13 \mathrm{~g}$ of $60 \%$ dispersion in oil, $3.3 \mathrm{mmol}, 1.1$ equiv) was added to a stirred solution trimethylsulfoxonium iodide $(0.86 \mathrm{~g}, 3.9 \mathrm{mmol}, 1.3$ equiv) in dry DMSO $(6 \mathrm{~mL})$ at room temperature under gentle flow of dry argon. Reaction mixture was stirred for 15-20 minutes until clear solution was formed (sometimes the formed ylide partially precipitates, which does not influence the reaction course. 4-Arylidene-1,2-dialkyl- $1 \mathrm{H}$-imidazol-5(4H)one ( $3 \mathrm{mmol}, 1$ equiv) was added as a solid in several portions during 5 minutes. The reaction was stirred for 1-2 hours at room temperature ( 4 hours for $\mathbf{1 b}$ preparation) and poured into separatory funnel with EtOAc $(30 \mathrm{~mL})$ and water $(30 \mathrm{~mL})$. The organic layer was separated and the aqueous layer was back-extracted with EtOAc $(10 \mathrm{~mL})$. Combined organic layers were washed with brine $(30 \mathrm{~mL})$, dried over anhydrous $\mathrm{Na}_{2} \mathrm{SO}_{4}$ and concentrated in vacuo. The residue was either recrystallized from hexane/EtOAc mixture or subjected to column chromatography on silica (eluent hexane/EtOAc or EtOAc) to give cyclopropane 1 in $32-74 \%$ yield.

\section{Cis-5,6-dimethyl-1-(4-methoxyphenyl)-4,6-diazaspiro[2.4]hept-4-en-7-one 1a}

Prepared from $2.38 \mathrm{~g}(10.7 \mathrm{mmol})$ of the starting arylideneimidazolone. Recrystallization of the crude product from $\mathrm{Et}_{2} \mathrm{O} / \mathrm{EtOAc} 3 / 2$ mixture gave $1.57 \mathrm{~g}(6.4 \mathrm{mmol}, 60 \%)$ of cyclopropane $1 \mathrm{a}$ as a single diastereomer. Beige crystals, $\mathrm{mp}=132-133^{\circ} \mathrm{C}\left(\mathrm{Et}_{2} \mathrm{O} / \mathrm{EtOAc}\right) ; \mathrm{R}_{f}=0.18$ (EtOAc, UV/vanillin viz.).

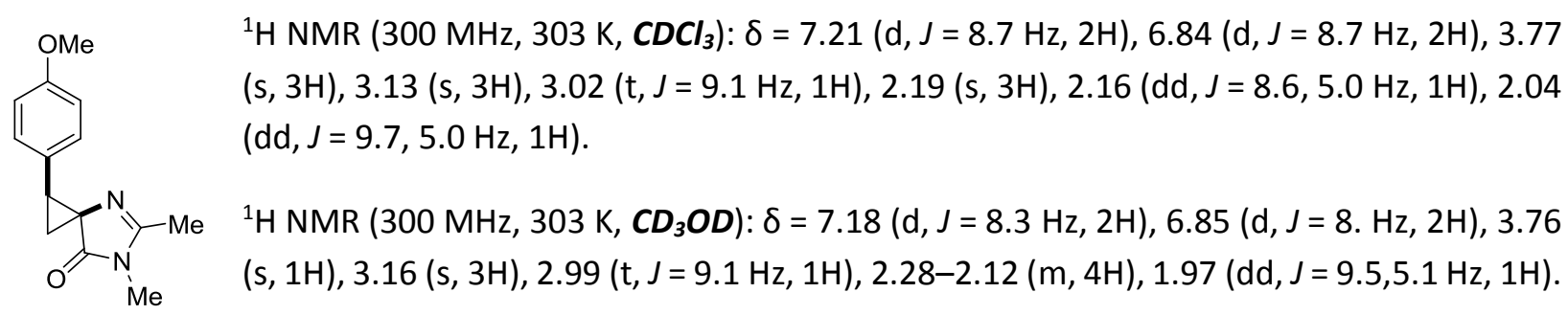

${ }^{13} \mathrm{C} \mathrm{NMR}\left(75 \mathrm{MHz}, 303 \mathrm{~K}, \mathrm{CD}_{3} \mathrm{OD}\right): \delta=182.4(\mathrm{C}=\mathrm{O}), 164.1$ and $160.4(\mathrm{C}=\mathrm{N}$ and $\mathrm{C}-\mathrm{O}), 130.9(2 \times \mathrm{CH}), 128.6$ (C), $114.7(2 \times \mathrm{CH}), 57.1(\mathrm{C}), 55.7\left(\mathrm{CH}_{3}-\mathrm{O}\right), 36.7(\mathrm{CH}), 27.2\left(\mathrm{CH}_{3}\right), 22.9\left(\mathrm{CH}_{2}\right), 15.2\left(\mathrm{CH}_{3}\right)$.

HRMS (ESI) m/z: [M+H'] Calcd for $\mathrm{C}_{14} \mathrm{H}_{17} \mathrm{~N}_{2} \mathrm{O}_{2} 245.1285$, found 245.1277.

\section{Cis-5,6-dimethyl-1-(2-methoxyphenyl)-4,6-diazaspiro[2.4]hept-4-en-7-one 1b}

Prepared from $1.71 \mathrm{~g}(7.4 \mathrm{mmol})$ of the starting arylideneimidazolone. Recrystallization of the crude product from hexane/EtOAc 1/1 mixture gave $1.27 \mathrm{~g} \mathrm{(5.2} \mathrm{mmol,} \mathrm{70 \% )} \mathrm{of} \mathrm{cyclopropane} \mathbf{1 b}$ as a single diastereomer. Off-white crystals, $\mathrm{mp}=109-110^{\circ} \mathrm{C}$ (hexane/EtOAc); $\mathrm{R}_{f}=0.18$ (EtOAc, UV/vanillin viz.).

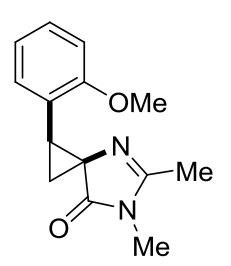

${ }^{1} \mathrm{H}$ NMR $\left(300 \mathrm{MHz}, 303 \mathrm{~K}, \mathrm{CDCl}_{3}\right): \delta=7.39-7.14(\mathrm{~m}, 2 \mathrm{H}), 6.94(\mathrm{t}, J=7.7 \mathrm{~Hz}, 1 \mathrm{H}), 6.81(\mathrm{~d}, J$ $=8.2 \mathrm{~Hz}, 1 \mathrm{H}), 3.73(\mathrm{~s}, 3 \mathrm{H}), 3.24(\mathrm{t}, J=9.2 \mathrm{~Hz}, 1 \mathrm{H}), 3.16(\mathrm{~s}, 3 \mathrm{H}), 2.24-2.16(\mathrm{~m}, 4 \mathrm{H}), 2.05(\mathrm{dd}$, $J=9.6,4.9 \mathrm{~Hz}, 1 \mathrm{H})$.

${ }^{13} \mathrm{C} \mathrm{NMR}\left(75 \mathrm{MHz}, 303 \mathrm{~K}, \mathrm{CDCl}_{3}\right): \delta=181.4(\mathrm{C}=\mathrm{O}), 160.3$ and $158.8(\mathrm{C}=\mathrm{N}$ and $\mathrm{C}-\mathrm{O}), 128.5$ $(\mathrm{CH}), 128.3(\mathrm{CH}), 124.7(\mathrm{C}), 120.5(\mathrm{CH}), 110.2(\mathrm{CH}), 56.1(\mathrm{C}), 55.5\left(\mathrm{CH}_{3}-\mathrm{O}\right), 31.1(\mathrm{CH}), 27.0$ $\left(\mathrm{CH}_{3}\right), 22.3\left(\mathrm{CH}_{2}\right), 15.8\left(\mathrm{CH}_{3}\right)$.

HRMS (ESI) m/z: [M+Na ${ }^{+}$Calcd for $\mathrm{C}_{14} \mathrm{H}_{16} \mathrm{~N}_{2} \mathrm{NaO}_{2}$ 267.1104, found 267.1107.

\section{Cis-5,6-dimethyl-1-(3,4,5-trimethoxyphenyl)-4,6-diazaspiro[2.4]hept-4-en-7-one 1c}

Prepared from $0.25 \mathrm{~g}(0.8 \mathrm{mmol})$ of the starting arylideneimidazolone. Column chromatography on silica (eluent EtOAc) and following recrystallization from minimum amount of ether/hexane gave $0.16 \mathrm{~g}(0.5$ 
$\mathrm{mmol}, 60 \%$ ) of cyclopropane 1c. Colorless crystals, $\mathrm{mp}=94-95^{\circ} \mathrm{C}$ (hexane/EtOAc), $\mathrm{R}_{f}=0.11$ (EtOAc/iPrOH $=9 / 1$, UV/vanillin viz.).<smiles>C=NC(=O)N(C)C(=O)C1CC1c1cc(OC)c(OC)c(OC)c1</smiles>

${ }^{1} \mathrm{H} \mathrm{NMR}\left(300 \mathrm{MHz}, 303 \mathrm{~K}, \mathrm{CDCl}_{3}\right): \delta=6.53(\mathrm{~s}, 2 \mathrm{H}), 3.84(\mathrm{~s}, 6 \mathrm{H}), 3.81(\mathrm{~s}, 3 \mathrm{H}), 3.15(\mathrm{~s}$, $3 \mathrm{H}), 3.00(\mathrm{t}, J=9.1 \mathrm{~Hz}, 1 \mathrm{H}), 2.23(\mathrm{~s}, 3 \mathrm{H}), 2.12(\mathrm{dd}, J=8.5,4.9 \mathrm{~Hz}, 1 \mathrm{H}), 2.03(\mathrm{dd}, J=$ 9.7, $4.9 \mathrm{~Hz}, 1 \mathrm{H})$.

${ }^{13} \mathrm{C} \mathrm{NMR}\left(75 \mathrm{MHz}, 303 \mathrm{~K}, \mathrm{CDCl}_{3}\right): \delta=181.0(\mathrm{C}=\mathrm{O}), 160.8(\mathrm{C}=\mathrm{N}), 153.0(2 \times \mathrm{C}), 137.1(\mathrm{C})$, $131.7(\mathrm{C}), 105.8(2 \times \mathrm{CH}), 60.9$ and $56.6\left(\mathrm{C}\right.$ and $\left.\mathrm{OCH}_{3}\right), 56.2\left(2 \times \mathrm{OCH}_{3}\right), 36.3(\mathrm{CH}), 27.1$ $\left(\mathrm{CH}_{3}\right), 23.7\left(\mathrm{CH}_{2}\right), 16.0\left(\mathrm{CH}_{3}\right)$.

HRMS (ESI) m/z: [M+H'] Calcd for $\mathrm{C}_{16} \mathrm{H}_{21} \mathrm{~N}_{2} \mathrm{O}_{4}$ 305.1496, found 305.1494.

\section{Cis-1-(3,4-dimethoxyphenyl)-5,6-dimethyl-4,6-diazaspiro[2.4]hept-4-en-7-one 1d}

Prepared from $1.72 \mathrm{~g}(6.6 \mathrm{mmol})$ of the starting arylideneimidazolone. Column chromatography on silica (eluent EtOAc) and following recrystallization from minimum amount of ether gave $1.25 \mathrm{~g}$ (4.6 mmol, 69\%) of cyclopropane $1 \mathrm{~d}$. Colorless crystals, $\mathrm{mp}=101-103^{\circ} \mathrm{C}$ (ether), $\mathrm{R}_{f}=0.14$ (EtOAc/iPrOH $=9 / 1$, UV/vanillin viz.).<smiles>COc1ccc(C2CC2C(=O)N(C)C(C)=O)cc1OC</smiles>

${ }^{1} \mathrm{H}$ NMR $\left(300 \mathrm{MHz}, 303 \mathrm{~K}, \mathrm{CDCl}_{3}\right): \delta=6.91-6.46(\mathrm{~m}, 3 \mathrm{H}), 3.87(\mathrm{~s}, 3 \mathrm{H}), 3.85(\mathrm{~s}, 3 \mathrm{H})$, $3.15(\mathrm{~s}, 3 \mathrm{H}), 3.02(\mathrm{t}, J=9.1 \mathrm{~Hz}, 1 \mathrm{H}), 2.21(\mathrm{~s}, 3 \mathrm{H}), 2.16(\mathrm{dd}, J=8.5,5.0 \mathrm{~Hz}, 1 \mathrm{H}), 2.04$ (dd, $J=9.7,5.0 \mathrm{~Hz}, 1 \mathrm{H}$ ).

${ }^{13} \mathrm{C} \mathrm{NMR}\left(75 \mathrm{MHz}, 303 \mathrm{~K}, \mathrm{CDCl}_{3}\right): \delta=181.1$ (C=O), 160.7 (C=N), 148.7 (C), $148.2(\mathrm{C})$, $128.5(\mathrm{C}), 120.3(\mathrm{CH}), 112.1(\mathrm{CH}), 111.1(\mathrm{CH}), 56.7$ and $56.0\left(\mathrm{C}\right.$ and $\left.2 \times \mathrm{OCH}_{3}\right), 36.0(\mathrm{CH})$, $27.1\left(\mathrm{CH}_{3}\right), 23.5\left(\mathrm{CH}_{2}\right), 16.0\left(\mathrm{CH}_{3}\right)$.

HRMS (ESI) m/z: [M+H'] Calcd for $\mathrm{C}_{15} \mathrm{H}_{19} \mathrm{~N}_{2} \mathrm{O}_{3} 275.1390$, found 275.1402.<smiles>COc1cc(C=O)ccc1OC(F)F</smiles>

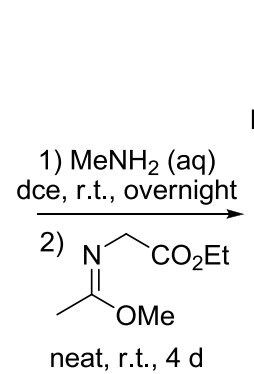

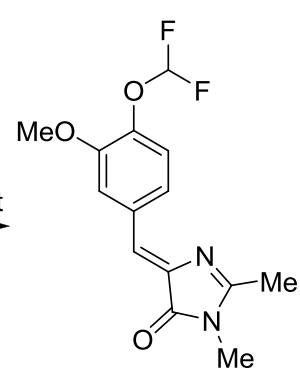

SI-1, $70 \%$

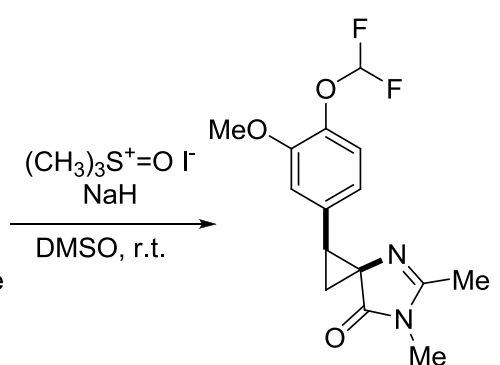

1 e, $57 \%$

(Z)-4-(4-(difluoromethoxy)-3-methoxybenzylidene)-1,2-dimethyl-1H-imidazol-5(4H)-one SI-1

The 4-(difluoromethoxy)-3-methoxybenzaldehyde (1.23 g, $6.1 \mathrm{mmol}, 1$ equiv) was dissolved in 1,2dichloroehtane $(10 \mathrm{~mL})$ and mixed with methylamine solution $(2.1 \mathrm{~mL}, 40 \%$ aqueous soln, $24.3 \mathrm{mmol}, 4$ equiv), and anhydrous $\mathrm{Na}_{2} \mathrm{SO}_{4}(10 \mathrm{~g})$. The mixture was stirred for $24 \mathrm{~h}$ at room temperature. The resulting yellow solution was filtered (solid was washed with extra 1,2-dichloroethane) and dried over the additional $\mathrm{Na}_{2} \mathrm{SO}_{4}$. The solvent was evaporated and ethyl((1-ethoxy)amino)acetate $(1.16 \mathrm{~g}, 6.7 \mathrm{mmol}, 1.1$ equiv) was added to the neat oily residue. The mixture was stirred for $4 \mathrm{~d}$ at room temperature upon which the reaction mixture mostly solidified. Volatiles were then evaporated and the residue was recrystallized from ether to give pure $\mathbf{S I - 1}(1.26 \mathrm{~g}, 4.3 \mathrm{mmol}, 70 \%)$ as yellow crystals. 


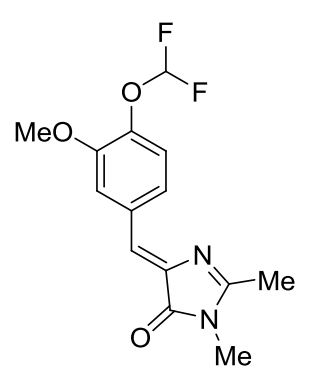

$\mathrm{mp}=125-126^{\circ} \mathrm{C}\left(\mathrm{Et}_{2} \mathrm{O}\right), \mathrm{R}_{f}=0.50$ (EtOAc, UV/vanillin viz.).

${ }^{1} \mathrm{H}$ NMR $\left(300 \mathrm{MHz}, 303 \mathrm{~K}, \mathrm{CDCl}_{3}\right): \delta=8.01(\mathrm{~d}, J=1.9 \mathrm{~Hz}, 1 \mathrm{H}), 7.54(\mathrm{dd}, J=8.4,1.9 \mathrm{~Hz}$, 1H), $7.14(\mathrm{~d}, J=8.4 \mathrm{~Hz}, 1 \mathrm{H}), 7.00(\mathrm{~s}, 1 \mathrm{H}), 6.59(\mathrm{t}, J=74.9 \mathrm{~Hz}, 1 \mathrm{H}), 3.93(\mathrm{~s}, 3 \mathrm{H}), 3.17(\mathrm{~s}$, $3 \mathrm{H}), 2.36(\mathrm{~s}, 3 \mathrm{H})$.

${ }^{13} \mathrm{C} \mathrm{NMR}\left(75 \mathrm{MHz}, 303 \mathrm{~K}, \mathrm{CDCl}_{3}\right): \delta=170.7(\mathrm{C}=0), 163.1(\mathrm{C}=\mathrm{N}), 150.9(\mathrm{C}), 141.4(\mathrm{t}, j=$ $2.8 \mathrm{~Hz}, \mathrm{C}), 139.0(\mathrm{C}), 132.8(\mathrm{C}), 126.0(\mathrm{CH}), 125.8(\mathrm{CH}), 121.8(\mathrm{CH}), 116.1(\mathrm{t}, \mathrm{J}=260.4$ $\left.\mathrm{Hz}, \mathrm{CF}_{2} \mathrm{H}\right), 115.7(\mathrm{CH}), 56.1\left(\mathrm{CH}_{3}\right), 26.7\left(\mathrm{CH}_{3}\right), 15.9\left(\mathrm{CH}_{3}\right)$.

HRMS (ESI) m/z: [M+H'] Calcd for $\mathrm{C}_{14} \mathrm{H}_{15} \mathrm{~F}_{2} \mathrm{~N}_{2} \mathrm{O}_{3} 297.1045$, found 297.1041.

\section{Cis-5,6-dimethyl-1-(4-(difluoromethoxy)-3-methoxyphenyl)-4,6-diazaspiro[2.4]hept-4-en-7-one 1e}

Prepared from $0.50 \mathrm{~g}(1.7 \mathrm{mmol})$ of the starting arylideneimidazolone. Column chromatography on silica (eluent EtOAc) gave $0.30 \mathrm{~g}(1.0 \mathrm{mmol}, 57 \%)$ of cyclopropane $1 \mathrm{e}$. Beige solid, $\mathrm{mp}=118-120{ }^{\circ} \mathrm{C} ; \mathrm{R}_{f}=0.13$ (EtOAc, UV/vanillin viz.)<smiles>COc1cc(C2CC23N=C(C)N(C)C3=O)ccc1OC(F)F</smiles>

${ }^{1} \mathrm{H} \mathrm{NMR}\left(700 \mathrm{MHz}, 303 \mathrm{~K}, \mathrm{CDCl}_{3}\right): \delta=8.07(\mathrm{~d}, J=8.2 \mathrm{~Hz}, 1 \mathrm{H}), 6.93(\mathrm{~d}, J=2.0 \mathrm{~Hz}, 1 \mathrm{H})$, $6.86(\mathrm{dd}, J=8.2,2.0 \mathrm{~Hz}, 1 \mathrm{H}), 6.50(\mathrm{t}, J=75.4 \mathrm{~Hz}, 1 \mathrm{H}), 3.86(\mathrm{~s}, 3 \mathrm{H}), 3.15(\mathrm{~s}, 3 \mathrm{H}), 3.00$ $(\mathrm{t}, J=9.0 \mathrm{~Hz}, 1 \mathrm{H}), 2.22(\mathrm{~s}, 3 \mathrm{H}), 2.11(\mathrm{dd}, J=8.4,5.0 \mathrm{~Hz}, 1 \mathrm{H}), 2.05(\mathrm{dd}, J=9.7,5.0 \mathrm{~Hz}$, $1 \mathrm{H})$.

${ }^{13} \mathrm{C}$ NMR (175 MHz, $\left.303 \mathrm{~K}, \mathrm{CDCl}_{3}\right): \delta=180.9(\mathrm{C}=\mathrm{O}), 160.9(\mathrm{C}=\mathrm{N}), 150.9\left(\mathrm{C}_{\mathrm{Ar}}\right), 139.1(\mathrm{t}$, $\left.J=3.1 \mathrm{~Hz}, \mathrm{C}_{\mathrm{Ar}}\right), 135.1\left(\mathrm{C}_{\mathrm{Ar}}\right), 122.1\left(\mathrm{CH}_{\mathrm{Ar}}\right), 121.0\left(\mathrm{CH}_{\mathrm{Ar}}\right), 116.4\left(\mathrm{t}, J=259.5 \mathrm{~Hz}, \mathrm{CHF}_{2}\right)$, $113.4\left(\mathrm{CH}_{\mathrm{Ar}}\right), 55.6(\mathrm{C}), 56.1\left(\mathrm{CH}_{3}\right), 35.5(\mathrm{CH}), 27.1\left(\mathrm{CH}_{3}\right), 23.7\left(\mathrm{CH}_{2}\right), 16.0\left(\mathrm{CH}_{3}\right)$.

HRMS (ESI) m/z: [M+H'] Calcd for $\mathrm{C}_{15} \mathrm{H}_{17} \mathrm{~F}_{2} \mathrm{~N}_{2} \mathrm{O}_{3} 311.1202$, found 311.1203 .

\section{Cis-5,6-dimethyl-1-phenyl-4,6-diazaspiro[2.4]hept-4-en-7-one 1f}

Prepared from $0.39 \mathrm{~g}$ (1.9 mmol) of the starting arylideneimidazolone. Column chromatography on silica (eluent EtOAc) gave $0.29 \mathrm{~g}$ (1.4 mmol, 69\%) of cyclopropane 1f. Beige crystals, $\mathrm{mp}=127-129^{\circ} \mathrm{C}\left(\mathrm{Et}_{2} \mathrm{O}\right) ; \mathrm{R}_{f}$ $=0.20$ (EtOAc, UV/vanillin viz.)

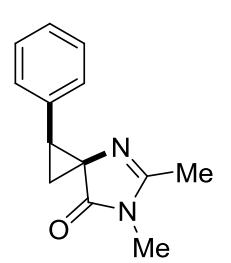

${ }^{1} \mathrm{H}$ NMR (300 MHz, $\left.303 \mathrm{~K}, \mathrm{CDCl}_{3}\right): \delta=7.42-7.15(\mathrm{~m}, 5 \mathrm{H}), 3.15(\mathrm{~s}, 3 \mathrm{H}), 3.06$ (dd, $J=9.7,8.5$ $\mathrm{Hz}, 1 \mathrm{H}), 2.29-2.17(\mathrm{~m}, 4 \mathrm{H}), 2.07(\mathrm{dd}, J=9.7,5.0 \mathrm{~Hz}, 1 \mathrm{H})$.

${ }^{13} \mathrm{C}$ NMR (75 MHz, $\left.303 \mathrm{~K}, \mathrm{CDCl}_{3}\right): \delta=180.9(\mathrm{C}=\mathrm{O}), 161.0(\mathrm{C}=\mathrm{N}), 135.9(\mathrm{C}), 128.5(2 \times \mathrm{CH})$, $128.4(2 \times \mathrm{CH}), 127.1(\mathrm{CH}), 56.7(\mathrm{C}), 36.2(\mathrm{CH}), 27.1\left(\mathrm{CH}_{3}\right), 23.3\left(\mathrm{CH}_{2}\right), 16.0\left(\mathrm{CH}_{3}\right)$.

HRMS (ESI) m/z: $\left[\mathrm{M}+\mathrm{H}^{+}\right]$Calcd for $\mathrm{C}_{13} \mathrm{H}_{15} \mathrm{~N}_{2} \mathrm{O} 215.1179$, found 215.1175

\section{Cis-1-(4-bromophenyl)-5,6-dimethyl-4,6-diazaspiro[2.4]hept-4-en-7-one $1 \mathrm{~g}$}

Prepared from $0.62 \mathrm{~g}(2.2 \mathrm{mmol})$ of the starting arylideneimidazolone. Column chromatography on silica (eluent EtOAc) gave $0.49 \mathrm{~g}(1.6 \mathrm{mmol}, 75 \%)$ of cyclopropane $1 \mathrm{~g}$. Brown crystals, $\mathrm{mp}=146-147^{\circ} \mathrm{C}\left(\mathrm{Et}_{2} \mathrm{O}\right)$; $\mathrm{R}_{f}=0.21$ (EtOAc, UV/vanillin viz.) 


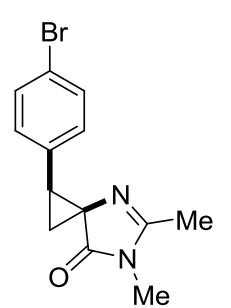

${ }^{1} \mathrm{H}$ NMR $\left(300 \mathrm{MHz}, 303 \mathrm{~K}, \mathrm{CDCl}_{3}\right): \delta=7.42(\mathrm{~d}, J=8.5 \mathrm{~Hz}, 2 \mathrm{H}), 7.18(\mathrm{~d}, J=8.5 \mathrm{~Hz}, 2 \mathrm{H}), 3.15$ (s, 3H), $2.99(\mathrm{t}, J=9.0 \mathrm{~Hz}, 1 \mathrm{H}), 2.23(\mathrm{~s}, 3 \mathrm{H}), 2.17(\mathrm{dd}, J=8.5,5.1 \mathrm{~Hz}, 1 \mathrm{H}), 2.07$ (dd, J = 9.6, $5.1 \mathrm{~Hz}, 1 \mathrm{H})$.

${ }^{13} \mathrm{C} \mathrm{NMR}\left(75 \mathrm{MHz}, 303 \mathrm{~K}, \mathrm{CDCl}_{3}\right): \delta=180.4(\mathrm{C}=\mathrm{O}), 161.5(\mathrm{C}=\mathrm{N}), 134.9(\mathrm{C}), 131.4(2 \times \mathrm{CH})$, $130.3(2 \times \mathrm{CH}), 121.1(\mathrm{C}), 56.3(\mathrm{C}), 33.4(\mathrm{CH}), 27.1\left(\mathrm{CH}_{3}\right), 23.3\left(\mathrm{CH}_{2}\right), 15.9\left(\mathrm{CH}_{3}\right)$.

HRMS (ESI) $\mathrm{m} / \mathrm{z}$ : $\left[\mathrm{M}+\mathrm{H}^{+}\right]$Calcd for $\mathrm{C}_{13} \mathrm{H}_{14}{ }^{79} \mathrm{BrN}_{2} \mathrm{O}$ 293.0284; for $\mathrm{C}_{13} \mathrm{H}_{14}{ }^{81} \mathrm{BrN}_{2} \mathrm{O}$ 295.0265; found 293.0301, 295.0282.

\section{Cis-5,6-dimethyl-1-(2-thienyl)-4,6-diazaspiro[2.4]hept-4-en-7-one $1 \mathrm{f}$}

Prepared from $0.30 \mathrm{~g}(1.4 \mathrm{mmol})$ of the starting arylideneimidazolone. Column chromatography on silica (eluent EtOAc) gave $0.15 \mathrm{~g}(0.7 \mathrm{mmol}, 48 \%)$ of cyclopropane 1f. Orange solid, $\mathrm{mp}=133-135^{\circ} \mathrm{C}$ (hexane/EtOAc); $R_{f}=0.20$ (EtOAc, UV/vanillin viz.)

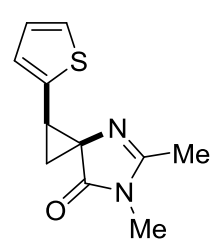

${ }^{1} \mathrm{H} \mathrm{NMR}\left(300 \mathrm{MHz}, 303 \mathrm{~K}, \mathrm{CDCl}_{3}\right): \delta=7.16(\mathrm{dd}, J=5.0,1.4 \mathrm{~Hz}, 1 \mathrm{H}), 6.99-6.89(\mathrm{~m}, 2 \mathrm{H}), 3.19$ $(\mathrm{t}, J=8.9 \mathrm{~Hz}, 1 \mathrm{H}), 3.15(\mathrm{~s}, 3 \mathrm{H}), 2.24(\mathrm{~s}, 3 \mathrm{H}), 2.12(\mathrm{~d}, J=8.9 \mathrm{~Hz}, 2 \mathrm{H})$.

${ }^{13} \mathrm{C} \mathrm{NMR}\left(75 \mathrm{MHz}, 303 \mathrm{~K}, \mathrm{CDCl}_{3}\right): \delta=180.6(\mathrm{C}=\mathrm{O}), 161.3(\mathrm{C}=\mathrm{N}), 139.6(\mathrm{C}), 126.9(\mathrm{CH}), 126.3$ $(\mathrm{CH}), 124.6(\mathrm{CH}), 56.6(\mathrm{C}), 31.1(\mathrm{CH}), 27.1\left(\mathrm{CH}_{3}\right), 24.9\left(\mathrm{CH}_{2}\right), 16.0\left(\mathrm{CH}_{3}\right)$.

HRMS (ESI) m/z: [M+H'] Calcd for $\mathrm{C}_{11} \mathrm{H}_{13} \mathrm{~N}_{2} \mathrm{OS} 221.0743$, found 221.0751.

\section{Cis-5,6-dimethyl-1-(2-furyl)-4,6-diazaspiro[2.4]hept-4-en-7-one 1g}

Prepared from $0.43 \mathrm{~g}(2.3 \mathrm{mmol})$ of the starting arylideneimidazolone. Column chromatography on silica (eluent EtOAc) gave $0.32 \mathrm{~g}(1.6 \mathrm{mmol}, 70 \%)$ of cyclopropane $1 \mathrm{~g}$. Yellowish solid, $\mathrm{mp}=126-128^{\circ} \mathrm{C}$ (hexane/Et ${ }_{2} \mathrm{O}$ ); $\mathrm{R}_{f}=0.18$ (EtOAc, UV/vanillin viz.)

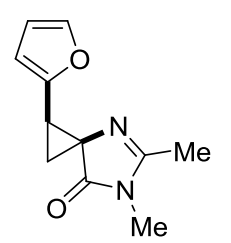

${ }^{1} \mathrm{H} \mathrm{NMR}\left(300 \mathrm{MHz}, 303 \mathrm{~K}, \mathrm{CDCl}_{3}\right): \delta=7.35(\mathrm{~d}, J=1.9 \mathrm{~Hz}, 1 \mathrm{H}), 6.32(\mathrm{dd}, J=3.2,1.9 \mathrm{~Hz}, 1 \mathrm{H})$, $6.23(\mathrm{~d}, J=3.2 \mathrm{~Hz}, 1 \mathrm{H}), 3.13(\mathrm{~s}, 3 \mathrm{H}), 3.00(\mathrm{dd}, J=9.8,8.2 \mathrm{~Hz}, 1 \mathrm{H}), 2.5(\mathrm{dd}, J=8.2,4.8 \mathrm{~Hz}$, $1 \mathrm{H}), 2.24(\mathrm{~s}, 3 \mathrm{H}), 2.03(\mathrm{dd}, J=9.8,4.8 \mathrm{~Hz}, 1 \mathrm{H})$.

${ }^{13} \mathrm{CNMR}\left(75 \mathrm{MHz}, 303 \mathrm{~K}, \mathrm{CDCl}_{3}\right): \delta=180.4(\mathrm{C}=\mathrm{O}), 161.4(\mathrm{C}=\mathrm{N}), 150.5(\mathrm{C}), 142.2(\mathrm{CH}), 110.7$ $(\mathrm{CH}), 108.0(\mathrm{CH}), 56.2(\mathrm{C}), 28.7(\mathrm{CH}), 27.1\left(\mathrm{CH}_{3}\right), 21.6\left(\mathrm{CH}_{2}\right), 16.0\left(\mathrm{CH}_{3}\right)$.

HRMS (ESI) m/z: $\left[\mathrm{M}+\mathrm{H}^{+}\right]$Calcd for $\mathrm{C}_{11} \mathrm{H}_{13} \mathrm{~N}_{2} \mathrm{O}_{2}$ 205.0972, found 205.0968.

\section{Cis-1-(anthracen-9-yl)-5,6-dimethyl-4,6-diazaspiro[2.4]hept-4-en-7-one 1h}

Prepared from $0.32 \mathrm{~g}(1.2 \mathrm{mmol})$ of the starting arylideneimidazolone. Two successive column chromatography on silica (eluent for 1 column: hexane/EtOAc $=1 / 1 \rightarrow$ EtOAc; 2 column: hexane/EtOAc $=$ $1 / 2 \rightarrow$ EtOAc) gave $0.11 \mathrm{~g}(0.6 \mathrm{mmol}, 54 \%)$ of cyclopropane $1 \mathrm{~h}$. Brown solid, $\mathrm{mp}=128-131^{\circ} \mathrm{C}$ (hexane $/ \mathrm{Et}_{2} \mathrm{O}$, with decomposition), $\mathrm{R}_{f}=0.30$ (EtOAc, UV/vanillin viz.)

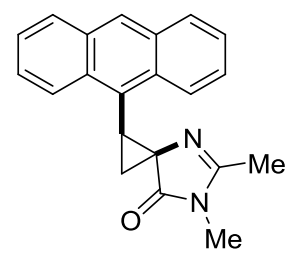

${ }^{1} \mathrm{H} \mathrm{NMR}\left(700 \mathrm{MHz}, 303 \mathrm{~K}, \mathrm{CDCl}_{3}\right): \delta=8.40(\mathrm{~s}, 1 \mathrm{H}), 8.12-7.90(\mathrm{br} \mathrm{m}, 2 \mathrm{H}), 7.97$ (dd, $J=$ 7.0, $2.4 \mathrm{~Hz}, 2 \mathrm{H}), 7.43-7.37(\mathrm{~m}, 4 \mathrm{H}), 3.70(\mathrm{t}, J=9.2 \mathrm{~Hz}, 1 \mathrm{H}), 3.31(\mathrm{~s}, 3 \mathrm{H}), 2.69(\mathrm{dd}, J=$ 9.6, 4.7 Hz, 1H), $2.37(\mathrm{dd}, J=8.9,4.7 \mathrm{~Hz}, 1 \mathrm{H}), 2.02(\mathrm{~s}, 3 \mathrm{H})$.

${ }^{13} \mathrm{C} \mathrm{NMR}\left(75 \mathrm{MHz}, 303 \mathrm{~K}, \mathrm{CDCl}_{3}\right): \delta=181.9(\mathrm{C}=\mathrm{O}), 160.4(\mathrm{C}=\mathrm{N}), 131.6(2 \times \mathrm{C}), 131.5$ $(2 \times \mathrm{C}), 129.2(2 \times \mathrm{CH}), 128.0(\mathrm{C}), 127.9(\mathrm{CH}), 125.3(2 \times \mathrm{CH}), 125.0(2 \times \mathrm{CH}), 124.8(2 \times \mathrm{CH})$, $56.1(\mathrm{C}), 31.0(\mathrm{CH}), 27.3$ and $26.0\left(\mathrm{CH}_{3}\right.$ and $\left.\mathrm{CH}_{2}\right), 15.4\left(\mathrm{CH}_{3}\right)$. 
HRMS (ESI) m/z: [M+H'] Calcd for $\mathrm{C}_{21} \mathrm{H}_{19} \mathrm{~N}_{2} \mathrm{O}$ 315.1492, found 315.1495.

Cis-5-ethyl-1-(4-methoxyphenyl)-6-methyl-4,6-diazaspiro[2.4]hept-4-en-7-one 1k

Prepared from $0.91 \mathrm{~g}(3.7 \mathrm{mmol})$ of the starting arylideneimidazolone. Recrystallization of the crude product from EtOAc/hexane $1 / 3$ mixture gave $0.60 \mathrm{~g}(2.3 \mathrm{mmol}, 63 \%)$ of cyclopropane $1 \mathbf{k}$ as a single diastereomer. Off-white crystals, $\mathrm{mp}=97-98^{\circ} \mathrm{C}$ (hexane/EtOAc); $\mathrm{R}_{f}=0.41$ (EtOAc, UV/vanillin viz.).

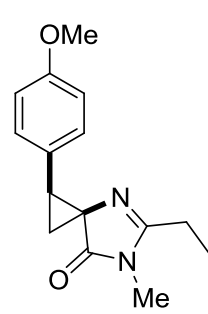

${ }^{1} \mathrm{H}$ NMR (300 MHz, $\left.303 \mathrm{~K}, \mathrm{CDCl}_{3}\right): \delta=7.23(\mathrm{~d}, J=8.7 \mathrm{~Hz}, 2 \mathrm{H}), 6.83(\mathrm{~d}, J=8.7 \mathrm{~Hz}, 2 \mathrm{H}$ ), 3.78 $(\mathrm{s}, 3 \mathrm{H}), 3.13(\mathrm{~s}, 3 \mathrm{H}), 2.99(\mathrm{t}, J=9.1 \mathrm{~Hz}, 1 \mathrm{H}), 2.48(\mathrm{q}, J=7.56 \mathrm{~Hz}, 2 \mathrm{H}), 2.13(\mathrm{dd}, J=8.5,4.8$ $\mathrm{Hz}, 1 \mathrm{H}), 2.05(\mathrm{dd}, J=9.8,4.8 \mathrm{~Hz}, 1 \mathrm{H}), 1.21(\mathrm{t}, J=7.5 \mathrm{~Hz}, 3 \mathrm{H}$ ).

${ }^{13} \mathrm{C}$ NMR (75 MHz, $303 \mathrm{~K}, \mathrm{CDCl}_{3}$ ): $\delta=181.5$ (C=O), 164.5 and 158.6 ( $\mathrm{C}=\mathrm{N}$ and $\left.\mathrm{C}-\mathrm{O}\right), 129.7$ $(2 \times \mathrm{CH}), 128.3(\mathrm{C}), 113.7(2 \times \mathrm{CH}), 56.5(\mathrm{C}), 55.4\left(\mathrm{CH}_{3}-\mathrm{O}\right), 35.8(\mathrm{CH}), 26.9\left(\mathrm{CH}_{3}\right), 23.8\left(\mathrm{CH}_{2}\right)$, $22.7\left(\mathrm{CH}_{2}\right), 10.1\left(\mathrm{CH}_{3}\right)$.

HRMS (ESI) m/z: [M+H'] Calcd for $\mathrm{C}_{15} \mathrm{H}_{19} \mathrm{~N}_{2} \mathrm{O}_{2} 259.1441$, found 259.1441.

\section{Cis-6-cyclohexyl-1-(4-methoxyphenyl)-5-methyl-4,6-diazaspiro[2.4]hept-4-en-7-one 1l}

Prepared from $0.41 \mathrm{~g}(1.4 \mathrm{mmol})$ of the starting arylideneimidazolone. Column chromatography on silica (eluent hexane/EtOAc $=1 / 1 \rightarrow 1 / 2)$ gave $0.34 \mathrm{~g}(1.1 \mathrm{mmol}, 74 \%)$ of cyclopropane 1 . Off-white crystals, $\mathrm{mp}=137-139^{\circ} \mathrm{C}$ (hexane $/$ EtOAc); $R_{f}=0.13$ (hexane $/$ EtOAc $=1 / 1$, UV/vanillin viz.).

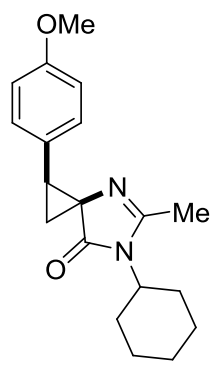

${ }^{1} \mathrm{H}$ NMR $\left(300 \mathrm{MHz}, 303 \mathrm{~K}, \mathrm{CDCl}_{3}\right): \delta=7.23(\mathrm{~d}, J=8.7 \mathrm{~Hz}, 2 \mathrm{H}), 6.85(\mathrm{~d}, J=8.7 \mathrm{~Hz}, 2 \mathrm{H}), 3.78$ $(\mathrm{s}, 3 \mathrm{H}), 3.69(\mathrm{tt}, J=12.3,3.8 \mathrm{~Hz}, 1 \mathrm{H}), 2.96(\mathrm{t}, J=9.1 \mathrm{~Hz}, 1 \mathrm{H}), 2.25(\mathrm{~s}, 3 \mathrm{H}), 2.14-1.95(\mathrm{~m}$, $4 \mathrm{H}), 1.93-1.81(\mathrm{~m}, 2 \mathrm{H}), 1.80-1.60(\mathrm{~m}, 3 \mathrm{H}), 1.40-1.15(\mathrm{~m}, 3 \mathrm{H})$.

$\left.{ }^{13} \mathrm{C} \mathrm{NMR} \mathrm{(75} \mathrm{MHz,} 303 \mathrm{~K}, \mathrm{CDCl}_{3}\right): \delta=181.5(\mathrm{C}=\mathrm{O}), 161.4$ and 159.1 ( $\mathrm{C}=\mathrm{N}$ and $\left.\mathrm{C}-\mathrm{O}\right), 130.0$ $(2 \times \mathrm{CH}), 128.7(\mathrm{C}), 114.2(2 \times \mathrm{CH}), 56.8(\mathrm{C}), 55.8$ and $54.6\left(\mathrm{CH}\right.$ and $\left.\mathrm{CH}_{3}-\mathrm{O}\right), 36.1(\mathrm{CH}), 31.0$ $\left(2 \times \mathrm{CH}_{2}\right), 26.6\left(2 \times \mathrm{CH}_{2}\right), 25.6\left(\mathrm{CH}_{2}\right), 24.2\left(\mathrm{CH}_{2}\right), 17.9\left(\mathrm{CH}_{3}\right)$.

HRMS (ESI) m/z: [M+H'] Calcd for or $\mathrm{C}_{19} \mathrm{H}_{25} \mathrm{~N}_{2} \mathrm{O}_{2}$ 313.1911, found 313.1914.

\section{Cis-6-benzyl-1-(4-methoxyphenyl)-5-methyl-4,6-diazaspiro[2.4]hept-4-en-7-one 1m}

Prepared from $1.00 \mathrm{~g}(3.3 \mathrm{mmol})$ of the starting arylideneimidazolone. Recrystallization from hexane/EtOAc $=4 / 1$ gave $0.71 \mathrm{~g}(2.2 \mathrm{mmol}, 68 \%)$ of cyclopropane $1 \mathrm{~m}$ as a single diastereomer. Off-white crystals, $m p=108-109^{\circ} \mathrm{C}$ (hexane/EtOAc); $\mathrm{R}_{f}=0.42$ (EtOAc, UV/vanillin viz.).

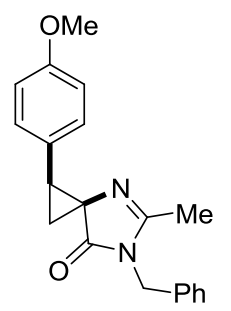

${ }^{1} \mathrm{H} \mathrm{NMR}\left(300 \mathrm{MHz}, 303 \mathrm{~K}, \mathrm{CDCl}_{3}\right): \delta=7.48-7.00(\mathrm{~m}, 7 \mathrm{H}), 6.85(\mathrm{~d}, J=8.7 \mathrm{~Hz}, 2 \mathrm{H}), 4.85-4.68$ $(\mathrm{m}, 2 \mathrm{H}), 3.79(\mathrm{~s}, 3 \mathrm{H}), 3.11(\mathrm{t}, J=9.1 \mathrm{~Hz}, 1 \mathrm{H}), 2.28-1.95(\mathrm{~m}, 5 \mathrm{H})$.

${ }^{13} \mathrm{C}$ NMR $\left(75 \mathrm{MHz}, 303 \mathrm{~K}, \mathrm{CDCl}_{3}\right): \delta=181.1(\mathrm{C}=\mathrm{O}), 160.5$ and 158.7 ( $\mathrm{C}=\mathrm{N}$ and $\left.\mathrm{C}-\mathrm{O}\right), 136.3$ (C), $129.6(2 \times \mathrm{CH}), 129.1(2 \times \mathrm{CH}), 128.0(\mathrm{CH}$ and $\mathrm{C}), 127.2(2 \times \mathrm{CH}), 113.8(2 \times \mathrm{CH}), 56.6$ $\left(\mathrm{OCH}_{3}\right), 55.4(\mathrm{C}), 44.3\left(\mathrm{CH}_{2}\right), 35.8(\mathrm{CH}), 23.7\left(\mathrm{CH}_{2}\right), 16.4\left(\mathrm{CH}_{3}\right)$.

HRMS (ESI) m/z: [M+H'] Calcd for $\mathrm{C}_{20} \mathrm{H}_{21} \mathrm{~N}_{2} \mathrm{O}_{2} 321.1598$, found 321.1593 . 


\section{Reaction series}

General procedure: Anhydrous $\mathrm{TsOH}(57 \mathrm{mg}, 0.33 \mathrm{mmol}, 1.1$ equiv) was added to a stirred solution of cyclopropane 1 ( $0.30 \mathrm{mmol}, 1$ equiv) and aldehyde 2 ( $0.90 \mathrm{mmol}, 3.0$ equiv) in anhydrous $\mathrm{CH}_{2} \mathrm{Cl}_{2}$ (3.0 mL) at indicated temperature (A color of the solution color immediately changed to a deep yellow, orange or red depending on the aryl group substitution). Reaction mixture was stirred for $0.5-24 \mathrm{~h}$ (TLC control), quenched with TMG ( $57 \mu \mathrm{L}, 51 \mathrm{mg}, 0.45 \mathrm{mmol}, 1.5$ equiv) and stirred for additional $10 \mathrm{~min}$. The resulting mixture was subjected to column chromatography on silica (eluent hexane/EtOAc, 1/1 $\rightarrow$ EtOAc $\rightarrow$ EtOAc/iPrOH, 9/1) to furnish target 1,3-diazaspiro[4.4]non-1-en-4-ones 3' (less polar, 7-52\% yield) and 3 (more polar, $25-57 \%$ yield).

$\left(5 S^{*}, 6 R^{*}, 8 R^{*}\right)$-2,3-Dimethyl-8-(4-methoxyphenyl)-6-(3,4,5-trimethoxyphenyl)-7-oxa-1,3diazaspiro[4.4]non-1-en-4-one 3aa

Yield $67 \mathrm{mg}(0.15 \mathrm{mmol}, 51 \%)$. Colorless crystals; $\mathrm{mp}=136-138^{\circ} \mathrm{C}$ (hexane/EtOAc); $\mathrm{R}_{f}=0.06$ (EtOAc, UV/vanillin viz.).

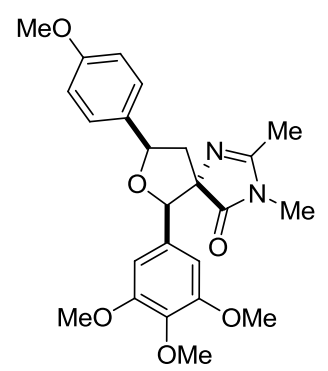

${ }^{1} \mathrm{H}$ NMR $\left(700 \mathrm{MHz}, 303 \mathrm{~K}, \mathrm{CDCl}_{3}\right): \delta=7.62(\mathrm{~d}, J=8.7 \mathrm{~Hz}, 2 \mathrm{H}), 6.95(\mathrm{~d}, J=8.7 \mathrm{~Hz}, 2 \mathrm{H})$, $6.60(\mathrm{~s}, 2 \mathrm{H}), 5.23(\mathrm{dd}, J=8.8,7.4 \mathrm{~Hz}, 1 \mathrm{H}), 5.04(\mathrm{~s}, 1 \mathrm{H}), 3.83(\mathrm{~s}, 9 \mathrm{H}), 3.81(\mathrm{~s}, 3 \mathrm{H}), 2.72$ $(\mathrm{s}, 3 \mathrm{H}), 2.67(\mathrm{dd}, J=13.3,7.4 \mathrm{~Hz}, 1 \mathrm{H}), 2.42(\mathrm{dd}, J=13.3,8.8 \mathrm{~Hz}, 1 \mathrm{H}), 2.17(\mathrm{~s}, 3 \mathrm{H})$.

${ }^{13} \mathrm{C} \mathrm{NMR}\left(175 \mathrm{MHz}, 303 \mathrm{~K}, \mathrm{CDCl}_{3}\right): \delta=182.2$ (C=O), 162.0 (C), 158.7 (C), 152.8 (2×O$\left.\mathrm{C}_{\mathrm{Ar}}\right), 137.8(\mathrm{C}), 132.9(\mathrm{C}), 131.4(\mathrm{C}), 128.7\left(2 \times \mathrm{CH}_{\mathrm{Ar}}\right), 114.1\left(2 \times \mathrm{CH}_{\mathrm{Ar}}\right), 103.4\left(2 \times \mathrm{CH}_{\mathrm{Ar}}\right)$, $86.4(\mathrm{CH}-\mathrm{O}), 80.7(\mathrm{CH}-\mathrm{O}), 80.0(\mathrm{C}), 61.0\left(\mathrm{OCH}_{3}\right), 56.3\left(2 \times \mathrm{OCH}_{3}\right), 55.4\left(\mathrm{OCH}_{3}\right), 44.4$ $\left(\mathrm{CH}_{2}\right), 26.3\left(\mathrm{CH}_{3}\right), 15.7\left(\mathrm{CH}_{3}\right)$.

HRMS (ESI) m/z: [M+H'] Calcd for $\mathrm{C}_{24} \mathrm{H}_{29} \mathrm{~N}_{2} \mathrm{O}_{6} 441.2020$, found 441.1998.

$\left(5 R^{*}, 6 R^{*}, 8 R^{*}\right)-2,3-D i m e t h y l-8-(4-m e t h o x y p h e n y l)-6-(3,4,5-t r i m e t h o x y p h e n y l)-7-o x a-1,3-$ diazaspiro[4.4]non-1-en-4-one 3'aa

Yield $53 \mathrm{mg}(0.12 \mathrm{mmol}, 40 \%)$. Colorless crystals; $\mathrm{mp}=127-129^{\circ} \mathrm{C}\left(\mathrm{Et}_{2} \mathrm{O}\right) ; \mathrm{R}_{f}=0.18(\mathrm{EtOAc}, \mathrm{UV} / \mathrm{vanillin}$ viz.).

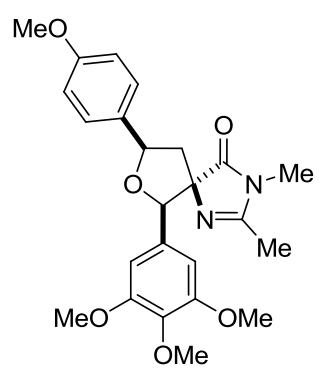

${ }^{1} \mathrm{H} \mathrm{NMR}\left(700 \mathrm{MHz}, 303 \mathrm{~K}, \mathrm{CDCl}_{3}\right): \delta=7.55(\mathrm{~d}, J=8.6 \mathrm{~Hz}, 2 \mathrm{H}), 6.92(\mathrm{~d}, J=8.6 \mathrm{~Hz}, 2 \mathrm{H})$, $6.49(\mathrm{~s}, 2 \mathrm{H}), 5.25(\mathrm{t}, J=7.9 \mathrm{~Hz}, 1 \mathrm{H}), 5.04(\mathrm{~s}, 1 \mathrm{H}), 3.82(\mathrm{~s}, 3 \mathrm{H}), 3.80(\mathrm{~s}, 9 \mathrm{H}), 3.00(\mathrm{~s}$, $3 \mathrm{H}), 2.85(\mathrm{dd}, J=13.3,8.4 \mathrm{~Hz}, 1 \mathrm{H}), 2.25(\mathrm{dd}, J=13.3,7.5 \mathrm{~Hz}, 1 \mathrm{H}), 1.96(\mathrm{~s}, 3 \mathrm{H})$.

${ }^{1} \mathrm{H}$ NMR $\left(300 \mathrm{MHz}, 303 \mathrm{~K}\right.$, DMSO-d $\left.d_{6}\right): \delta=7.53(\mathrm{~d}, J=8.7 \mathrm{~Hz}, 2 \mathrm{H}), 6.96(\mathrm{~d}, J=8.7 \mathrm{~Hz}$, $2 \mathrm{H}), 6.44(\mathrm{~s}, 2 \mathrm{H}), 5.21(\mathrm{dd}, J=8.5,6.8 \mathrm{~Hz}, 1 \mathrm{H}), 4.90(\mathrm{~s}, 1 \mathrm{H}), 3.75(\mathrm{~s}, 3 \mathrm{H}), 3.70(\mathrm{~s}, 6 \mathrm{H})$, $3.63(\mathrm{~s}, 3 \mathrm{H}), 2.95(\mathrm{~s}, 3 \mathrm{H}), 2.76(\mathrm{dd}, J=13.0,8.5 \mathrm{~Hz}, 1 \mathrm{H}), 2.02(\mathrm{dd}, J=13.0,6.8 \mathrm{~Hz}$, $1 \mathrm{H}), 1.95(\mathrm{~s}, 3 \mathrm{H})$.

${ }^{13} \mathrm{C}$ NMR $\left(75 \mathrm{MHz}, 303 \mathrm{~K}, \mathrm{DMSO}-\boldsymbol{d}_{6}\right): \delta=181.9$ (C=0), 160.0 (C), 158.9 (C), $152.2\left(2 \times 0-\mathrm{C}_{\mathrm{Ar}}\right), 136.9$ (C), 134.0 (C), $131.6(\mathrm{C}), 128.1\left(2 \times \mathrm{CH}_{\mathrm{Ar}}\right), 113.8\left(2 \times \mathrm{CH}_{\mathrm{Ar}}\right), 103.6\left(2 \times \mathrm{CH}_{\mathrm{Ar}}\right), 87.2(\mathrm{CH}-\mathrm{O}), 79.1(\mathrm{CH}-\mathrm{O}), 78.4(\mathrm{C}), 60.0$ $\left(\mathrm{OCH}_{3}\right), 55.8\left(2 \times \mathrm{OCH}_{3}\right), 55.1\left(\mathrm{OCH}_{3}\right), 44.5\left(\mathrm{CH}_{2}\right), 26.3\left(\mathrm{CH}_{3}\right), 14.7\left(\mathrm{CH}_{3}\right)$.

HRMS (ESI) m/z: [M+H'] Calcd for $\mathrm{C}_{24} \mathrm{H}_{29} \mathrm{~N}_{2} \mathrm{O}_{6} 441.2020$, found 441.2015. 
$\left(5 S^{*}, 6 R^{*}, 8 R^{*}\right)-6-(3,4-D i m e t h o x y p h e n y l)-2,3-d i m e t h y l-8-(4-m e t h o x y p h e n y l)-7-o x a-1,3-$ diazaspiro[4.4]non-1-en-4-one 3ab

Yield $55 \mathrm{mg}(0.13 \mathrm{mmol}, 45 \%)$. Colorless needles; $\mathrm{mp}=125-127^{\circ} \mathrm{C}\left(\mathrm{Et}_{2} \mathrm{O}\right) ; \mathrm{R}_{f}=0.03$ (EtOAc, UV/vanillin viz.).

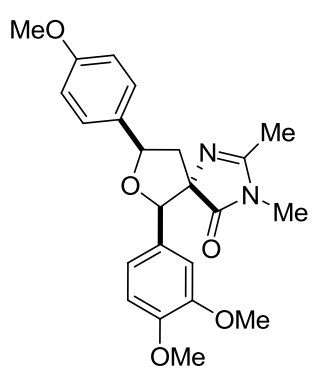

${ }^{1} \mathrm{H} \mathrm{NMR}\left(700 \mathrm{MHz}, 303 \mathrm{~K}, \mathrm{CDCl}_{3}\right): \delta=7.62(\mathrm{~d}, J=8.6 \mathrm{~Hz}, 2 \mathrm{H}), 6.95(\mathrm{~d}, J=8.6 \mathrm{~Hz}, 2 \mathrm{H})$, $6.93(\mathrm{~d}, J=1.9 \mathrm{~Hz}, 1 \mathrm{H}), 6.89(\mathrm{dd}, J=8.4,1.8 \mathrm{~Hz}, 1 \mathrm{H}), 6.77(\mathrm{~d}, J=8.3 \mathrm{~Hz}, 1 \mathrm{H}), 5.23$ $(\mathrm{dd}, J=8.9,7.3 \mathrm{~Hz}, 1 \mathrm{H}), 5.07(\mathrm{~s}, 1 \mathrm{H}), 3.86(\mathrm{~s}, 3 \mathrm{H}), 3.84(\mathrm{~s}, 3 \mathrm{H}), 3.82(\mathrm{~s}, 3 \mathrm{H}), 2.69(\mathrm{~s}$, $3 \mathrm{H}), 2.65(\mathrm{dd}, J=13.3,7.3 \mathrm{~Hz}, 1 \mathrm{H}), 2.43(\mathrm{dd}, J=13.3,8.9 \mathrm{~Hz}, 1 \mathrm{H}), 2.17(\mathrm{~s}, 3 \mathrm{H})$.

${ }^{13} \mathrm{C}$ NMR (175 MHz, $\left.303 \mathrm{~K}, \mathrm{CDCl}_{3}\right): \delta=182.3(\mathrm{C}=0), 161.8$ (C), 159.6 (C), 148.8 (C), $148.4(\mathrm{C}), 132.9(\mathrm{C}), 128.6\left(2 \times \mathrm{CH}_{\mathrm{Ar}}\right), 128.4(\mathrm{C}), 118.5\left(\mathrm{CH}_{\mathrm{Ar}}\right), 114.0\left(2 \times \mathrm{CH}_{\mathrm{Ar}}\right), 110.5$ $\left(\mathrm{CH}_{\mathrm{Ar}}\right), 109.6\left(\mathrm{CH}_{\mathrm{Ar}}\right), 86.3(\mathrm{CH}-\mathrm{O}), 80.5(\mathrm{CH}-\mathrm{O}), 80.1(\mathrm{C}), 56.0\left(\mathrm{OCH}_{3}\right), 55.9\left(\mathrm{OCH}_{3}\right)$, $55.4\left(\mathrm{OCH}_{3}\right), 44.5\left(\mathrm{CH}_{2}\right), 26.3\left(\mathrm{CH}_{3}\right), 15.7\left(\mathrm{CH}_{3}\right)$.

HRMS (ESI) m/z: [M+H'] Calcd for $\mathrm{C}_{23} \mathrm{H}_{27} \mathrm{~N}_{2} \mathrm{O}_{5} 411.1914$, found 411.1914.

$\left(5 R^{*}, 6 R^{*}, 8 R^{*}\right)-6-(3,4-D i m e t h o x y p h e n y l)-2,3-d i m e t h y l-8-(4-m e t h o x y p h e n y l)-7-o x a-1,3-$ diazaspiro[4.4]non-1-en-4-one 3'ab

Yield $49 \mathrm{mg}(0.12 \mathrm{mmol}, 40 \%)$. Colorless crystals; $\mathrm{mp}=111-112{ }^{\circ} \mathrm{C}\left(\mathrm{Et}_{2} \mathrm{O}\right) ; \mathrm{R}_{f}=0.15(\mathrm{EtOAc}, \mathrm{UV} /$ vanillin viz.).

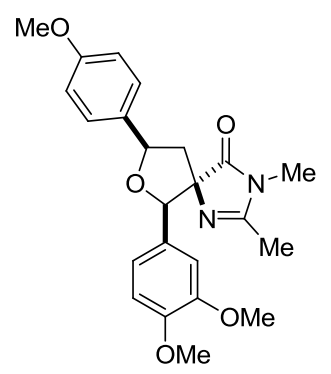

${ }^{1} \mathrm{H} \mathrm{NMR}\left(700 \mathrm{MHz}, 303 \mathrm{~K}, \mathrm{CDCl}_{3}\right): \delta=7.56(\mathrm{~d}, J=8.6 \mathrm{~Hz}, 2 \mathrm{H}), 6.92(\mathrm{~d}, J=8.6 \mathrm{~Hz}, 2 \mathrm{H})$, $6.86(\mathrm{~d}, J=1.9 \mathrm{~Hz}, 1 \mathrm{H}), 6.81(\mathrm{dd}, J=8.3,1.9 \mathrm{~Hz}, 1 \mathrm{H}), 6.75(\mathrm{~d}, J=8.3 \mathrm{~Hz}, 1 \mathrm{H}), 5.24$ $(\mathrm{dd}, J=8.4,7.4 \mathrm{~Hz}, 1 \mathrm{H}), 5.05(\mathrm{~s}, 1 \mathrm{H}), 3.84^{5}(\mathrm{~s}, 3 \mathrm{H}), 3.84^{2}(\mathrm{~s}, 3 \mathrm{H}), 3.81(\mathrm{~s}, 3 \mathrm{H}), 2.97(\mathrm{~s}$, $3 \mathrm{H}), 2.86(\mathrm{dd}, J=13.3,8.4 \mathrm{~Hz}, 1 \mathrm{H}), 2.25(\mathrm{dd}, J=13.3,7.4 \mathrm{~Hz}, 1 \mathrm{H}), 1.94(\mathrm{~s}, 3 \mathrm{H})$.

${ }^{13} \mathrm{C} \mathrm{NMR}\left(75 \mathrm{MHz}, 303 \mathrm{~K}, \mathrm{CDCl}_{3}\right): \delta=181.7$ (C=0), 159.6 (C), 159.5 (C), 148.7 (C), $148.3(\mathrm{C}), 133.5(\mathrm{C}), 128.5\left(2 \times \mathrm{CH}_{\mathrm{Ar}}\right), 128.0(\mathrm{C}), 119.1\left(\mathrm{CH}_{\mathrm{Ar}}\right), 114.0\left(2 \times \mathrm{CH}_{\mathrm{Ar}}\right), 110.3$ $\left(\mathrm{CH}_{\mathrm{Ar}}\right), 110.1\left(\mathrm{CH}_{\mathrm{Ar}}\right), 89.0(\mathrm{CH}-\mathrm{O}), 80.4(\mathrm{CH}-\mathrm{O}), 79.3(\mathrm{C}), 56.0\left(\mathrm{OCH}_{3}\right), 55.9\left(\mathrm{OCH}_{3}\right)$, $55.4\left(\mathrm{OCH}_{3}\right), 44.8\left(\mathrm{CH}_{2}\right), 26.8\left(\mathrm{CH}_{3}\right), 15.2\left(\mathrm{CH}_{3}\right)$.

HRMS (ESI) m/z: [M+H'] Calcd for $\mathrm{C}_{23} \mathrm{H}_{27} \mathrm{~N}_{2} \mathrm{O}_{5} 411.1914$, found 411.1913. 
Yield $57 \mathrm{mg}$ (0.15 mmol, 50\%). Viscous oil; $\mathrm{R}_{f}=0.10$ (EtOAc, UV/vanillin viz.).

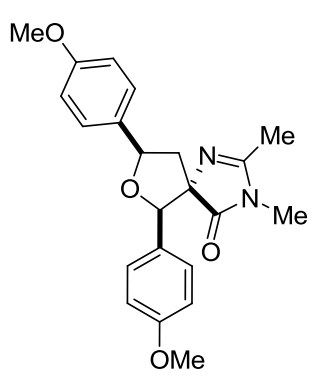

$15.7\left(\mathrm{CH}_{3}\right)$

${ }^{1} \mathrm{H}$ NMR $\left(700 \mathrm{MHz}, 303 \mathrm{~K}, \mathrm{CDCl}_{3}\right): \delta=7.61(\mathrm{~d}, J=8.6 \mathrm{~Hz}, 2 \mathrm{H}), 7.27(\mathrm{~d}, J=8.6 \mathrm{~Hz}, 2 \mathrm{H})$, $6.94(\mathrm{~d}, J=8.6 \mathrm{~Hz}, 2 \mathrm{H}), 6.80(\mathrm{~d}, J=8.6 \mathrm{~Hz}, 2 \mathrm{H}), 5.23(\mathrm{dd}, J=9.1,7.1 \mathrm{~Hz}, 1 \mathrm{H}), 5.09(\mathrm{~s}$, $1 \mathrm{H}), 3.82(\mathrm{~s}, 3 \mathrm{H}), 3.77(\mathrm{~s}, 3 \mathrm{H}), 2.68(\mathrm{~s}, 3 \mathrm{H}), 2.63(\mathrm{dd}, J=13.3,7.1 \mathrm{~Hz}, 1 \mathrm{H}), 2.42(\mathrm{dd}, J$ $=13.3,9.1 \mathrm{~Hz}, 1 \mathrm{H}), 2.18(\mathrm{~s}, 3 \mathrm{H})$.

$\left.{ }^{13} \mathrm{C} \mathrm{NMR} \mathrm{(150} \mathrm{MHz,} 303 \mathrm{~K}, \mathrm{CDCl}_{3}\right): \delta=182.4$ (C=0), 161.7 (C), 159.6 (C), 159.4 (C), $133.9(\mathrm{C}), 128.5\left(2 \times \mathrm{CH}_{\mathrm{Ar}}\right), 128.1(\mathrm{C}), 127.3\left(2 \times \mathrm{CH}_{\mathrm{Ar}}\right), 114.0\left(2 \times \mathrm{CH}_{\mathrm{Ar}}\right), 113.3\left(2 \times \mathrm{CH}_{\mathrm{Ar}}\right)$, $86.2(\mathrm{CH}-\mathrm{O}), 80.5(\mathrm{CH}-\mathrm{O}), 80.1(\mathrm{C}), 55.4\left(\mathrm{OCH}_{3}\right), 55.2\left(\mathrm{OCH}_{3}\right), 44.6\left(\mathrm{CH}_{2}\right), 26.2\left(\mathrm{CH}_{3}\right)$,

HRMS (ESI) m/z: [M+H'] Calcd for $\mathrm{C}_{22} \mathrm{H}_{25} \mathrm{~N}_{2} \mathrm{O}_{4} 381.1809$, found 381.1810.

$\left(5 R^{*}, 6 R^{*}, 8 R^{*}\right)-6,8$-bis(4-methoxyphenyl)-2,3-dimethyl-7-oxa-1,3-diazaspiro[4.4]non-1-en-4-one 3'ac Yield $48 \mathrm{mg}(0.13 \mathrm{mmol}, 42 \%)$. Colorless solid, $\mathrm{mp}=129-130{ }^{\circ} \mathrm{C}\left(\mathrm{Et}_{2} \mathrm{O}\right) ; \mathrm{R}_{f}=0.35$ (EtOAc, UV/vanillin viz.).

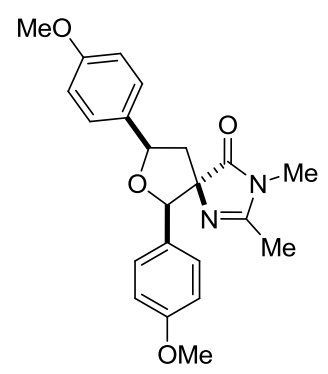
${ }^{1} \mathrm{H} \mathrm{NMR}\left(700 \mathrm{MHz}, 303 \mathrm{~K}, \mathrm{CDCl}_{3}\right): \delta=7.55(\mathrm{~d}, J=8.6 \mathrm{~Hz}, 2 \mathrm{H}), 7.20(\mathrm{~d}, J=8.6 \mathrm{~Hz}, 2 \mathrm{H})$, $6.92(\mathrm{~d}, J=8.6 \mathrm{~Hz}, 2 \mathrm{H}), 6.78(\mathrm{~d}, J=8.6 \mathrm{~Hz}, 2 \mathrm{H}), 5.24(\mathrm{t}, J=7.9 \mathrm{~Hz}, 1 \mathrm{H}), 5.06(\mathrm{~s}, 1 \mathrm{H})$, $3.81(\mathrm{~s}, 3 \mathrm{H}), 3.77(\mathrm{~s}, 3 \mathrm{H}), 2.96(\mathrm{~s}, 3 \mathrm{H}), 2.85(\mathrm{dd}, J=13.3,8.4 \mathrm{~Hz}, 1 \mathrm{H}), 2.44(\mathrm{dd}, J=$ $13.3,7.4 \mathrm{~Hz}, 1 \mathrm{H}), 1.91(\mathrm{~s}, 3 \mathrm{H})$.

${ }^{13} \mathrm{C} \mathrm{NMR}\left(150 \mathrm{MHz}, 303 \mathrm{~K}, \mathrm{CDCl}_{3}\right): \delta=183.0(\mathrm{C}=0), 159.5(\mathrm{C}), 159.3(2 \times \mathrm{C}), 133.8(\mathrm{C})$, $128.4\left(2 \times \mathrm{CH}_{\mathrm{Ar}}\right), 128.0\left(2 \times \mathrm{CH}_{\mathrm{Ar}}\right), 127.8(\mathrm{C}), 114.0\left(2 \times \mathrm{CH}_{\mathrm{Ar}}\right), 113.1\left(2 \times \mathrm{CH}_{\mathrm{Ar}}\right), 88.9(\mathrm{CH}-$ O), $80.4(\mathrm{CH}-\mathrm{O}), 79.4(\mathrm{C}), 55.4\left(\mathrm{OCH}_{3}\right), 55.3\left(\mathrm{OCH}_{3}\right), 44.9\left(\mathrm{CH}_{2}\right), 26.7\left(\mathrm{CH}_{3}\right), 15.2\left(\mathrm{CH}_{3}\right)$.

HRMS (ESI) m/z: [M+H'] Calcd for $\mathrm{C}_{22} \mathrm{H}_{25} \mathrm{~N}_{2} \mathrm{O}_{4} 381.1809$, found 381.1811. 

3ad

Yield $59 \mathrm{mg}$ (0.17 mmol, 56\%). Yellowish oil; $\mathrm{R}_{f}=0.10$ (EtOAc, UV/vanillin viz.).

$\mathrm{MeO}$<smiles>CC1=N[C@]2(CC(c3ccc(F)cc3)OC2c2ccccc2)C(=O)N1C</smiles>

${ }^{1} \mathrm{H} \mathrm{NMR}\left(700 \mathrm{MHz}, 303 \mathrm{~K}, \mathrm{CDCl}_{3}\right): \delta=7.61(\mathrm{~d}, J=8.4 \mathrm{~Hz}, 2 \mathrm{H}), 7.33(\mathrm{~d}, J=7.2 \mathrm{~Hz}, 2 \mathrm{H})$, $7.27(\mathrm{t}, J=7.4 \mathrm{~Hz}, 2 \mathrm{H}), 7.23(\mathrm{t}, J=7.2 \mathrm{~Hz}, 1 \mathrm{H}), 6.94(\mathrm{~d}, J=8.4 \mathrm{~Hz}, 2 \mathrm{H}), 5.25(\mathrm{dd}, J=$ 9.1, 7.1 Hz, 1H), $5.14(\mathrm{~s}, 1 \mathrm{H}), 3.82(\mathrm{~s}, 3 \mathrm{H}), 2.64(\mathrm{~s}, 3 \mathrm{H}), 2.63(\mathrm{dd}, J=13.2,7.1 \mathrm{~Hz}, 1 \mathrm{H})$, $2.43(\mathrm{dd}, J=13.2,9.1 \mathrm{~Hz}, 1 \mathrm{H}), 2.18(\mathrm{~s}, 3 \mathrm{H})$.

$\left.{ }^{13} \mathrm{C} \mathrm{NMR} \mathrm{(175} \mathrm{MHz,} 303 \mathrm{~K}, \mathrm{CDCl}_{3}\right): \delta=182.2$ (C=0), 161.8 (C), 159.6 (C), 136.1 (C), $132.8(\mathrm{C}), 128.5\left(2 \times \mathrm{CH}_{\mathrm{Ar}}\right), 128.1\left(\mathrm{CH}_{\mathrm{Ar}}\right), 127.8\left(2 \times \mathrm{CH}_{\mathrm{Ar}}\right), 125.9\left(2 \times \mathrm{CH}_{\mathrm{Ar}}\right), 114.0$ $\left(2 \times \mathrm{CH}_{\mathrm{Ar}}\right), 86.3(\mathrm{CH}-\mathrm{O}), 80.7(\mathrm{CH}-\mathrm{O}), 80.2(\mathrm{C}), 55.4\left(\mathrm{OCH}_{3}\right), 44.6\left(\mathrm{CH}_{2}\right), 26.1\left(\mathrm{CH}_{3}\right), 15.7\left(\mathrm{CH}_{3}\right)$.

HRMS (ESI) m/z: [M+H'] Calcd for $\mathrm{C}_{21} \mathrm{H}_{22} \mathrm{~N}_{2} \mathrm{O}_{3}$ 351.1703, found 351.1705.

$\left(5 R^{*}, 6 R^{*}, 8 R^{*}\right)-2,3-D i m e t h y l-8-(4-m e t h o x y p h e n y l)-6-p h e n y l-7-o x a-1,3-d i a z a s p i r o[4.4] n o n-1-e n-4-o n e$ 3'ad

Yield $32 \mathrm{mg}$ (0.09 mmol, 30\%). Colorless oil; $\mathrm{R}_{f}=0.42$ (EtOAc, UV/vanillin viz.).

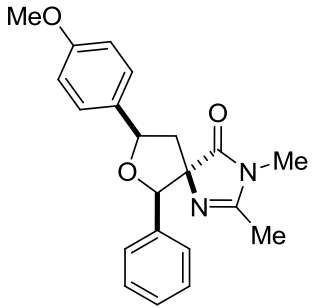

$\mathrm{MeO}$ $\left(\mathrm{CH}_{3}\right)$.

${ }^{1} \mathrm{H} \mathrm{NMR}\left(700 \mathrm{MHz}, 303 \mathrm{~K}, \mathrm{CDCl}_{3}\right): \delta=7.56(\mathrm{~d}, J=8.6 \mathrm{~Hz}, 2 \mathrm{H}), 7.28-7.19(\mathrm{~m}, 5 \mathrm{H})$, $6.93(\mathrm{~d}, J=8.6 \mathrm{~Hz}, 2 \mathrm{H}), 5.26(\mathrm{t}, J=7.9 \mathrm{~Hz}, 1 \mathrm{H}), 5.12(\mathrm{~s}, 1 \mathrm{H}), 3.81(\mathrm{~s}, 3 \mathrm{H}), 2.98(\mathrm{~s}, 3 \mathrm{H})$, $2.86(\mathrm{dd}, J=13.3,8.3 \mathrm{~Hz}, 1 \mathrm{H}), 2.25(\mathrm{dd}, J=13.3,7.5 \mathrm{~Hz}, 1 \mathrm{H}), 1.87(\mathrm{~s}, 3 \mathrm{H})$.

${ }^{13} \mathrm{C} \mathrm{NMR}\left(75 \mathrm{MHz}, 303 \mathrm{~K}, \mathrm{CDCl}_{3}\right): \delta=182.5$ (C=0), 160.2 (C), 159.5 (C), 135.5 (C), $133.3(\mathrm{C}), 128.5\left(2 \times \mathrm{CH}_{\mathrm{Ar}}\right), 128.0\left(\mathrm{CH}_{\mathrm{Ar}}\right), 127.8\left(2 \times \mathrm{CH}_{\mathrm{Ar}}\right), 126.4\left(2 \times \mathrm{CH}_{\mathrm{Ar}}\right), 114.0$ $\left(2 \times \mathrm{CH}_{\mathrm{Ar}}\right), 89.0(\mathrm{CH}-\mathrm{O}), 80.6(\mathrm{CH}-\mathrm{O}), 79.2(\mathrm{C}), 55.4\left(\mathrm{OCH}_{3}\right), 44.9\left(\mathrm{CH}_{2}\right), 26.8\left(\mathrm{CH}_{3}\right), 15.0$

HRMS (ESI) m/z: [M+H'] Calcd for $\mathrm{C}_{21} \mathrm{H}_{22} \mathrm{~N}_{2} \mathrm{O}_{3}$ 351.1703, found 351.1707. 


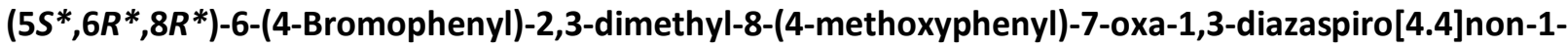
en-4-one 3ae

Yield $74 \mathrm{mg}(0.17 \mathrm{mmol}, 57 \%)$. Yellowish crystals, $\mathrm{mp}=68-70^{\circ} \mathrm{C}\left(\mathrm{Et}_{2} \mathrm{O}\right) ; \mathrm{R}_{f}=0.10$ (EtOAc, UV/vanillin viz.).

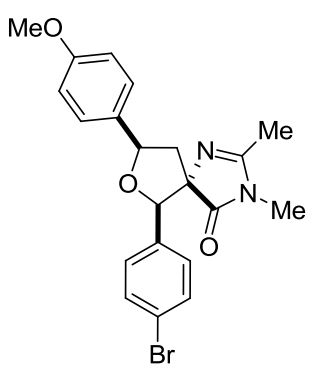

${ }^{1} \mathrm{H}$ NMR $\left(700 \mathrm{MHz}, 303 \mathrm{~K}, \mathrm{CDCl}_{3}\right): \delta=7.59(\mathrm{~d}, J=8.6 \mathrm{~Hz}, 2 \mathrm{H}), 7.40(\mathrm{~d}, J=8.4 \mathrm{~Hz}, 2 \mathrm{H})$, $7.22(\mathrm{~d}, J=8.4 \mathrm{~Hz}, 2 \mathrm{H}), 6.94(\mathrm{~d}, J=8.6 \mathrm{~Hz}, 2 \mathrm{H}), 5.24(\mathrm{dd}, J=9.1,7.1 \mathrm{~Hz}, 1 \mathrm{H}), 5.09(\mathrm{~s}$, $1 \mathrm{H}), 3.82(\mathrm{~s}, 3 \mathrm{H}), 2.71(\mathrm{~s}, 3 \mathrm{H}), 2.63(\mathrm{dd}, J=13.3,7.1 \mathrm{~Hz}, 1 \mathrm{H}), 2.42(\mathrm{dd}, J=13.3,9.1$ $\mathrm{Hz}, 1 \mathrm{H}), 2.19(\mathrm{~s}, 3 \mathrm{H})$.

$\left.{ }^{13} \mathrm{C} \mathrm{NMR} \mathrm{(175} \mathrm{MHz,} 303 \mathrm{~K}, \mathrm{CDCl}_{3}\right): \delta=182.0$ (C=O), 162.1 (C), 159.7 (C), 135.2 (C), $133.5(\mathrm{C}), 131.1\left(2 \times \mathrm{CH}_{\mathrm{Ar}}\right), 128.5\left(2 \times \mathrm{CH}_{\mathrm{Ar}}\right), 127.7\left(2 \times \mathrm{CH}_{\mathrm{Ar}}\right), 122.1(\mathrm{C}), 114.1\left(2 \times \mathrm{CH}_{\mathrm{Ar}}\right)$, 85.6 ( $\mathrm{CH}-\mathrm{O}), 80.8(\mathrm{CH}-\mathrm{O}), 80.0(\mathrm{C}), 55.4\left(\mathrm{OCH}_{3}\right), 44.8\left(\mathrm{CH}_{2}\right), 26.3\left(\mathrm{CH}_{3}\right), 15.7\left(\mathrm{CH}_{3}\right)$.

HRMS (ESI) m/z: $\left[\mathrm{M}+\mathrm{H}^{+}\right]$Calcd for $\mathrm{C}_{21} \mathrm{H}_{22}{ }^{79} \mathrm{BrN}_{2} \mathrm{O}_{3}$ 429.0808, for $\mathrm{C}_{21} \mathrm{H}_{22}{ }^{81} \mathrm{BrN}_{2} \mathrm{O}_{3}$ 431.0791, found 429.0809, 431.0791.

$\left(5 R^{*}, 6 R^{*}, 8 R^{*}\right)$-6-(4-Bromophenyl)-2,3-dimethyl-8-(4-methoxyphenyl)-7-oxa-1,3-diazaspiro[4.4]non-1en-4-one 3'ae

Yield $39 \mathrm{mg}$, (0.09 mmol, 30\%). Yellowish crystals, $\mathrm{mp}=123-125{ }^{\circ} \mathrm{C}$ (hexane/EtOAc); $\mathrm{R}_{f}=0.38$ (EtOAc, UV/vanillin viz.).

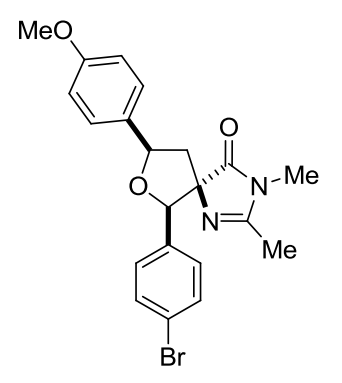

${ }^{1} \mathrm{H} \mathrm{NMR}\left(700 \mathrm{MHz}, 303 \mathrm{~K}, \mathrm{CDCl}_{3}\right): \delta=7.54(\mathrm{~d}, J=8.6 \mathrm{~Hz}, 2 \mathrm{H}), 7.38(\mathrm{~d}, J=8.4 \mathrm{~Hz}, 2 \mathrm{H})$, $7.15(\mathrm{~d}, J=8.4 \mathrm{~Hz}, 2 \mathrm{H}), 6.93(\mathrm{~d}, J=8.6 \mathrm{~Hz}, 2 \mathrm{H}), 5.25(\mathrm{t}, J=7.9 \mathrm{~Hz}, 1 \mathrm{H}), 5.07(\mathrm{~s}, 1 \mathrm{H})$, $3.82(\mathrm{~s}, 3 \mathrm{H}), 2.99(\mathrm{~s}, 3 \mathrm{H}), 2.85(\mathrm{dd}, J=13.3,8.4 \mathrm{~Hz}, 1 \mathrm{H}), 2.25(\mathrm{dd}, J=13.3,7.4 \mathrm{~Hz}$, $1 \mathrm{H}), 1.93(\mathrm{~s}, 3 \mathrm{H})$.

${ }^{13} \mathrm{C}$ NMR (175 MHz, $\left.303 \mathrm{~K}, \mathrm{CDCl}_{3}\right): \delta=182.7$ (C=0), 159.7 (C), 159.6 (C), 134.9 (C), $133.4(\mathrm{C}), 130.9\left(2 \times \mathrm{CH}_{\mathrm{Ar}}\right), 128.5\left(2 \times \mathrm{CH}_{\mathrm{Ar}}\right), 128.4\left(2 \times \mathrm{CH}_{\mathrm{Ar}}\right), 121.9(\mathrm{C}), 114.1\left(2 \times \mathrm{CH}_{\mathrm{Ar}}\right)$, $88.2(\mathrm{CH}-\mathrm{O}), 80.7(\mathrm{CH}-\mathrm{O}), 79.2(\mathrm{C}), 55.5\left(\mathrm{OCH}_{3}\right), 45.1\left(\mathrm{CH}_{2}\right), 26.8\left(\mathrm{CH}_{3}\right), 15.2\left(\mathrm{CH}_{3}\right)$.

HRMS (ESI+): calculated for $\mathrm{C}_{21} \mathrm{H}_{22}{ }^{79} \mathrm{BrN}_{2} \mathrm{O}_{3}\left(\mathrm{M}+\mathrm{H}^{+}\right)$: 429.0808 , for $\mathrm{C}_{21} \mathrm{H}_{22}{ }^{81} \mathrm{BrN}_{2} \mathrm{O}_{3}\left(\mathrm{M}+\mathrm{H}^{+}\right)$: 431.0791 , found 429.0807, 431.0791. 
$\left(5 S^{*}, 6 R^{*}, 8 R^{*}\right)-6-(4-C y a n o p h e n y l)-2,3-d i m e t h y l-8-(4-m e t h o x y p h e n y l)-7-o x a-1,3-d i a z a s p i r o[4.4] n o n-1-$ en-4-one 3 af

Yield $50 \mathrm{mg}(0.13 \mathrm{mmol}, 44 \%)$, Yellowish crystals; $\mathrm{mp}=157-159{ }^{\circ} \mathrm{C}$ (hexane/EtOAc); $\mathrm{R}_{f}=0.15$ (EtOAc/iPrOH = 9/1, UV/vanillin viz.).<smiles>COc1ccc(C2CC(c3ccc(C#N)cc3)OC(=O)C2C(=O)N(C)C)cc1</smiles>

HRMS (ESI) m/z: [M+H'] Calcd for $\mathrm{C}_{22} \mathrm{H}_{22} \mathrm{~N}_{3} \mathrm{O}_{3} 376.1656$, found 376.1661 . $15.7\left(\mathrm{CH}_{3}\right)$.

${ }^{1} \mathrm{H}$ NMR $\left(600 \mathrm{MHz}, 303 \mathrm{~K}, \mathrm{CDCl}_{3}\right): \delta=7.62-7.54(\mathrm{~m}, 4 \mathrm{H}), 7.45(\mathrm{~d}, J=8.0 \mathrm{~Hz}, 2 \mathrm{H}), 6.95$ (d, $J=8.7 \mathrm{~Hz}, 2 \mathrm{H}), 5.28(\mathrm{dd}, J=9.3,6.8 \mathrm{~Hz}, 1 \mathrm{H}), 5.20(\mathrm{~s}, 1 \mathrm{H}), 3.83(\mathrm{~s}, 3 \mathrm{H}), 2.73(\mathrm{~s}$, $3 \mathrm{H}), 2.66(\mathrm{dd}, J=13.2,6.8 \mathrm{~Hz}, 1 \mathrm{H}), 2.44(\mathrm{dd}, J=13.2,9.3 \mathrm{~Hz}, 1 \mathrm{H}), 2.25(\mathrm{~s}, 3 \mathrm{H})$.

${ }^{13} \mathrm{C} \mathrm{NMR}\left(150 \mathrm{MHz}, 303 \mathrm{~K}, \mathrm{CDCl}_{3}\right): \delta=181.3$ (C=0), 162.9 (C), 159.9 (C), 141.6 (C), $132.0(\mathrm{C}), 131.9\left(2 \times \mathrm{CH}_{\mathrm{Ar}}\right), 128.5\left(2 \times \mathrm{CH}_{\mathrm{Ar}}\right), 126.7\left(2 \times \mathrm{CH}_{\mathrm{Ar}}\right), 118.8(\mathrm{C}), 114.2\left(2 \times \mathrm{CH}_{\mathrm{Ar}}\right)$, $112.1(\mathrm{C} \equiv \mathrm{N}), 85.3(\mathrm{CH}-\mathrm{O}), 81.1(\mathrm{CH}-\mathrm{O}), 79.8(\mathrm{C}), 55.5\left(\mathrm{OCH}_{3}\right), 45.0\left(\mathrm{CH}_{2}\right), 26.4\left(\mathrm{CH}_{3}\right)$,

Minor diastereomer 3'af was not isolated. 
$\left(5 S^{*}, 6 R^{*}, 8 R^{*}\right)$-6-(2-bromo-5-methoxyphenyl)-2,3-dimethyl-8-(4-methoxyphenyl)-7-oxa-1,3diazaspiro[4.4]non-1-en-4-one 3ag and $\left(5 R^{*}, 6 R^{*}, 8 R^{*}\right)$-6-(2-bromo-5-methoxyphenyl)-2,3-dimethyl-8(4-methoxyphenyl)-7-oxa-1,3-diazaspiro[4.4]non-1-en-4-one 3'ag

Reaction performed on $0.5 \mathrm{mmol}$ for $2 \mathrm{~h}$ at r.t. Combined yield $170 \mathrm{mg}(0.37 \mathrm{mmol}, 74 \%), 2 / 1 \mathrm{mixture}$ of diastereomers 3ag/3'ag

Colorless crystals; $\mathrm{R}_{f}=0.40$ (EtOAc, UV/vanillin viz.).

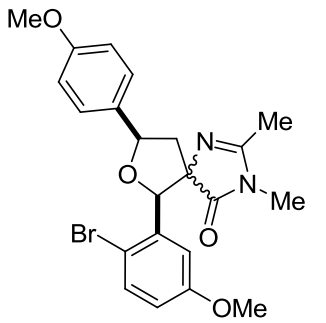

$\left.{ }^{1} \mathrm{H} \mathrm{NMR} \mathrm{(700} \mathrm{MHz,} 303 \mathrm{~K}, \mathrm{CDCl}_{3}\right): 3 a g$ (major): $\delta=7.52(\mathrm{~d}, J=8.6 \mathrm{~Hz}, 2 \mathrm{H}), 7.36(\mathrm{~d}, J$ $=3.2 \mathrm{~Hz}, 1 \mathrm{H}), 7.30(\mathrm{~d}, J \approx 8.5 \mathrm{~Hz}, 1 \mathrm{H}), 6.95(\mathrm{~d}, J=8.6 \mathrm{~Hz}, 2 \mathrm{H}), 6.68(\mathrm{dd}, J=8.5,3.2 \mathrm{~Hz}$, $1 \mathrm{H}), 5.51(\mathrm{~s}, 1 \mathrm{H}), 5.44(\mathrm{dd}, J=11.4,4.8 \mathrm{~Hz}, 1 \mathrm{H}), 3.82(\mathrm{~s}, 3 \mathrm{H}), 3.78(\mathrm{~s}, 3 \mathrm{H}), 2.91(\mathrm{~s}, 3 \mathrm{H})$, $2.44(\mathrm{dd}, J=12.4,11.4 \mathrm{~Hz}, 1 \mathrm{H}), 2.27(\mathrm{dd}, J=12.4,4.8 \mathrm{~Hz}, 1 \mathrm{H}), 2.24(\mathrm{~s}, 3 \mathrm{H}) ; 3$ 'ag (minor): $\delta=7.50(\mathrm{~d}, J=8.4 \mathrm{~Hz}, 2 \mathrm{H}), 7.41(\mathrm{~d}, J=3.1 \mathrm{~Hz}, 1 \mathrm{H}), 7.29(\mathrm{~d}, J \approx 8.2 \mathrm{~Hz}, 1 \mathrm{H})$, $6.94(\mathrm{~d}, J=8.4 \mathrm{~Hz}, 2 \mathrm{H}), 6.67(\mathrm{dd}, J=8.2,3.1 \mathrm{~Hz}, 1 \mathrm{H}), 5.42(\mathrm{~s}, 1 \mathrm{H}), 5.33(\mathrm{dd}, J=10.9$, $5.4 \mathrm{~Hz}, 1 \mathrm{H}), 3.82(\mathrm{~s}, 3 \mathrm{H}), 3.80(\mathrm{~s}, 3 \mathrm{H}), 3.05(\mathrm{~s}, 3 \mathrm{H}), 2.48(\mathrm{dd}, J=12.8,5.4 \mathrm{~Hz}, 1 \mathrm{H}), 2.37(\mathrm{dd}, J=12.8,10.9 \mathrm{~Hz}$, $1 \mathrm{H}), 2.01(\mathrm{~s}, 3 \mathrm{H})$.

${ }^{13} \mathrm{C}$ NMR (175 MHz, $303 \mathrm{~K}, \mathrm{CDCl}_{3}$ ): 3 ag (major): 180.6 (C=0), 161.5 (C), 159.7 (C), 158.8 (C), 138.4 (C), $132.7\left(\mathrm{CH}_{\mathrm{Ar}}\right), 131.7^{2}(\mathrm{C}), 128.1^{0}\left(2 \times \mathrm{CH}_{\mathrm{Ar}}\right), 115.5\left(\mathrm{CH}_{\mathrm{Ar}}\right), 115.3\left(\mathrm{CH}_{\mathrm{Ar}}\right), 114.1^{3}\left(2 \times \mathrm{CH}_{\mathrm{Ar}}\right), 111.6^{3}(\mathrm{C}), 85.1^{8}(\mathrm{CH}-$ O), $80.7(\mathrm{CH}-\mathrm{O}), 80.0(\mathrm{C}), 55.4^{5}\left(\mathrm{OCH}_{3}\right), 55.4^{3}\left(\mathrm{OCH}_{3}\right), 45.5^{3}\left(\mathrm{CH}_{2}\right), 26.7\left(\mathrm{CH}_{3}\right), 15.8\left(\mathrm{CH}_{3}\right) ; 3^{\prime}$ ag (minor): $\delta=$ 184.6 (C=O), 161.1 (C), 159.6 $6^{9}$ (C), 159.0 (C), 138.7 (C), $132.5\left(\mathrm{CH}_{\mathrm{Ar}}\right), 131.7^{9}$ (C), $128.0^{8}\left(2 \times \mathrm{CH}_{\mathrm{Ar}}\right), 115.0$ $\left(\mathrm{CH}_{\mathrm{Ar}}\right), 114.8\left(\mathrm{CH}_{\mathrm{Ar}}\right), 114.1^{2}\left(2 \times \mathrm{CH}_{\mathrm{Ar}}\right), 111.5^{9}(\mathrm{C}), 85.2^{5}(\mathrm{CH}-\mathrm{O}), 80.1(\mathrm{CH}-\mathrm{O}), 77.9(\mathrm{C}), 55.4^{2}\left(\mathrm{OCH}_{3}\right), 55.4^{1}$ $\left(\mathrm{OCH}_{3}\right), 45.4^{7}\left(\mathrm{CH}_{2}\right), 26.9\left(\mathrm{CH}_{3}\right), 15.4\left(\mathrm{CH}_{3}\right)$.

HRMS (ESI) m/z: [M+H'] Calcd for $\mathrm{C}_{22} \mathrm{H}_{23}{ }^{79} \mathrm{BrN}_{2} \mathrm{O}_{4} 459.0914$, for $\mathrm{C}_{22} \mathrm{H}_{23}{ }^{79} \mathrm{BrN}_{2} \mathrm{O}_{4}$ 461.0896, found 459.0914, 461.0900. 
$\left(5 S^{*}, 6 R^{*}, 8 R^{*}\right)$-2,3-dimethyl-8-(4-methoxyphenyl)-6-(naphthalen-1-yl)-7-oxa-1,3-diazaspiro[4.4]non-1en-4-one 3ah

Yield $48 \mathrm{mg}$ (0.12 mmol, 40\%). Viscous oil; $\mathrm{R}_{f}=0.12$ (EtOAc, UV/vanillin viz.).

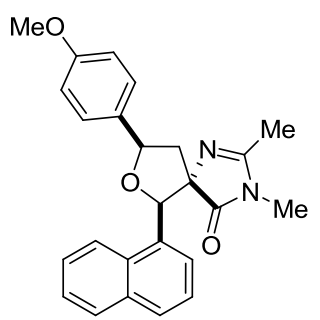

${ }^{1} \mathrm{H} \mathrm{NMR}\left(700 \mathrm{MHz}, 303 \mathrm{~K}, \mathrm{CDCl}_{3}\right): \delta=8.03(\mathrm{~d}, J=7.2 \mathrm{~Hz}, 1 \mathrm{H}), 7.82-7.77(\mathrm{~m}, 2 \mathrm{H}), 7.74$ $(\mathrm{dd}, J=8.1,1.2 \mathrm{~Hz}, 1 \mathrm{H}), 7.64-7.62(\mathrm{~m}, 1 \mathrm{H}), 7.63(\mathrm{~d}, J=8.5 \mathrm{~Hz}, 2 \mathrm{H}), 7.52(\mathrm{dd}, J=8.1$, $7.2 \mathrm{~Hz}, 1 \mathrm{H}$ ), 7.40 (ddd, $J=7.9,6.8,1.2 \mathrm{~Hz} 2 \mathrm{H}$ ), 7.36 (ddd, $J=8.4,6.8,1.4 \mathrm{~Hz}, 1 \mathrm{H}$ ), $6.97(\mathrm{~d}, J=8.5 \mathrm{~Hz}, 2 \mathrm{H}), 6.04(\mathrm{~s}, 1 \mathrm{H}), 5.43(\mathrm{dd}, J=9.9,6.1 \mathrm{~Hz}, 1 \mathrm{H}), 3.83(\mathrm{~s}, 3 \mathrm{H}), 2.55-$ $2.48(\mathrm{~m}, 2 \mathrm{H}), 2.36(\mathrm{~s}, 3 \mathrm{H}), 2.09(\mathrm{~s}, 3 \mathrm{H})$.

$\left.{ }^{13} \mathrm{C} \mathrm{NMR} \mathrm{(175} \mathrm{MHz,} 303 \mathrm{~K}, \mathrm{CDCl}_{3}\right): \delta=181.4$ (C=0), 162.2 (C), 159.7 (C), 133.4 (C), $132.7(\mathrm{C}), 132.2(\mathrm{C}), 130.3(\mathrm{C}), 128.9\left(\mathrm{CH}_{\mathrm{Ar}}\right), 128.4\left(2 \times \mathrm{CH}_{\mathrm{Ar}}\right), 128.1\left(\mathrm{CH}_{\mathrm{Ar}}\right), 125.5\left(\mathrm{CH}_{\mathrm{Ar}}\right), 125.2^{2}\left(\mathrm{CH}_{\mathrm{Ar}}\right), 125.1^{7}$ $\left(\mathrm{CH}_{\mathrm{Ar}}\right), 125.0\left(\mathrm{CH}_{\mathrm{Ar}}\right), 122.9\left(\mathrm{CH}_{\mathrm{Ar}}\right), 114.1\left(2 \times \mathrm{CH}_{\mathrm{Ar}}\right), 83.2(\mathrm{CH}-\mathrm{O}), 80.8(\mathrm{CH}-\mathrm{O}), 80.4(\mathrm{C}), 55.4\left(\mathrm{OCH}_{3}\right), 46.1$ $\left(\mathrm{CH}_{2}\right), 26.0\left(\mathrm{CH}_{3}\right), 15.7\left(\mathrm{CH}_{3}\right)$.

HRMS (ESI) m/z: [M+H'] Calcd for $\mathrm{C}_{25} \mathrm{H}_{25} \mathrm{~N}_{2} \mathrm{O}_{3} 401.1860$, found 401.1860 .

$\left(5 R^{*}, 6 R^{*}, 8 R^{*}\right)-2,3-d i m e t h y l-8-(4-m e t h o x y p h e n y l)-6-($ naphthalen-1-yl)-7-oxa-1,3-diazaspiro[4.4]non-1en-4-one 3'ah

Yield $38 \mathrm{mg}$ ( $0.10 \mathrm{mmol}, 32 \%)$. Colorless oil; $\mathrm{R}_{f}=0.36$ (EtOAc, UV/vanillin viz.).

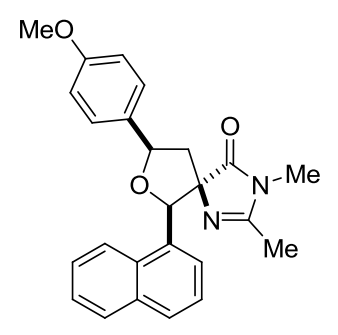

${ }^{1} \mathrm{H}$ NMR $\left(700 \mathrm{MHz}, 303 \mathrm{~K}, \mathrm{CDCl}_{3}\right): \delta=8.00(\mathrm{~d}, J=7.1 \mathrm{~Hz}, 1 \mathrm{H}), 7.82-7.79(\mathrm{~m}, 1 \mathrm{H})$, $7.74(\mathrm{~d}, J=8.1 \mathrm{~Hz}, 1 \mathrm{H}), 7.64-7.62(\mathrm{~m}, 1 \mathrm{H}), 7.60(\mathrm{~d}, J=8.6 \mathrm{~Hz}, 2 \mathrm{H}), 7.50(\mathrm{t}, J=7.7 \mathrm{~Hz}$, $1 \mathrm{H}), 7.42-7.37(\mathrm{~m}, 2 \mathrm{H}), 6.96(\mathrm{~d}, J=8.6 \mathrm{~Hz}, 2 \mathrm{H}), 5.92(\mathrm{~s}, 1 \mathrm{H}), 5.37(\mathrm{dd}, J=9.5,6.9 \mathrm{~Hz}$, $1 \mathrm{H}), 3.83(\mathrm{~s}, 3 \mathrm{H}), 2.80(\mathrm{~s}, 3 \mathrm{H}), 2.79(\mathrm{dd}, J=13.2,6.9 \mathrm{~Hz}, 1 \mathrm{H}), 2.40(\mathrm{dd}, J=13.2,9.5$ $\mathrm{Hz}, 1 \mathrm{H}), 1.56(\mathrm{~s}, 3 \mathrm{H})$.

${ }^{13} \mathrm{C} \mathrm{NMR}\left(175 \mathrm{MHz}, 303 \mathrm{~K}, \mathrm{CDCl}_{3}\right): \delta=184.2(\mathrm{C}=0), 159.7$ (C), $159.6(\mathrm{C}), 133.3$ (C), $132.7(\mathrm{C}), 132.5(\mathrm{C}), 130.3(\mathrm{C}), 128.9\left(\mathrm{CH}_{\mathrm{Ar}}\right), 128.3\left(2 \times \mathrm{CH}_{\mathrm{Ar}}\right), 127.9\left(\mathrm{CH}_{\mathrm{Ar}}\right), 125.6\left(\mathrm{CH}_{\mathrm{Ar}}\right), 125.4^{2}\left(\mathrm{CH}_{\mathrm{Ar}}\right), 125.3^{7}$ $\left(\mathrm{CH}_{\mathrm{Ar}}\right), 125.2\left(\mathrm{CH}_{\mathrm{Ar}}\right), 122.7\left(\mathrm{CH}_{\mathrm{Ar}}\right), 114.1\left(2 \times \mathrm{CH}_{\mathrm{Ar}}\right), 85.1(\mathrm{CH}-\mathrm{O}), 80.8(\mathrm{CH}-\mathrm{O}), 79.0(\mathrm{C}), 55.5\left(\mathrm{OCH}_{3}\right), 45.8$ $\left(\mathrm{CH}_{2}\right), 26.8\left(\mathrm{CH}_{3}\right), 14.8\left(\mathrm{CH}_{3}\right)$.

HRMS (ESI) m/z: [M+H'] Calcd for $\mathrm{C}_{25} \mathrm{H}_{25} \mathrm{~N}_{2} \mathrm{O}_{3} 401.1860$, found 401.1863 . 
$\left(5 S^{*}, 6 R^{*}, 8 R^{*}\right)$-2,3-dimethyl-8-(4-methoxyphenyl)-6-(thiophen-2-yl)-7-oxa-1,3-diazaspiro[4.4]non-1en-4-one 3ai

Yield $43 \mathrm{mg}$ (0.12 mmol, 40\%). Brown oil; $\mathrm{R}_{f}=0.08$ (EtOAc, UV/vanillin viz.).

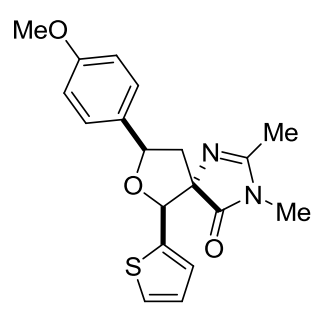

${ }^{1} \mathrm{H}$ NMR $\left(700 \mathrm{MHz}, 303 \mathrm{~K}, \mathrm{CDCl}_{3}\right): \delta=7.52(\mathrm{~d}, J=8.6 \mathrm{~Hz}, 2 \mathrm{H}), 7.20(\mathrm{dd}, J=5.0,1.3$ $\mathrm{Hz}, 1 \mathrm{H}), 6.98(\mathrm{dt}, J=3.3,1.1 \mathrm{~Hz}, 1 \mathrm{H}), 6.95-6.92(\mathrm{~m}, 3 \mathrm{H}), 5.36(\mathrm{~d}, J=1.0 \mathrm{~Hz}, 1 \mathrm{H}), 5.24$ $(\mathrm{dd}, J=9.2,6.9 \mathrm{~Hz}, 1 \mathrm{H}), 3.81(\mathrm{~s}, 3 \mathrm{H}), 2.79(\mathrm{~s}, 3 \mathrm{H}), 2.61(\mathrm{dd}, J=13.2,6.9 \mathrm{~Hz}, 1 \mathrm{H}), 2.45$ (dd, $J=13.2,9.2 \mathrm{~Hz}, 1 \mathrm{H}), 2.22(\mathrm{~s}, 3 \mathrm{H})$.

${ }^{13} \mathrm{C} \mathrm{NMR} \mathrm{(175} \mathrm{MHz,} 303 \mathrm{~K}, \mathrm{CDCl}_{3}$ ): $\delta=181.8$ (C=O), 162.4 (C), 159.7 (C), 138.7 (C), $132.5(\mathrm{C}), 128.4\left(2 \times \mathrm{CH}_{\mathrm{Ar}}\right), 126.5\left(\mathrm{CH}_{\mathrm{Ar}}\right), 124.8\left(\mathrm{CH}_{\mathrm{Ar}}\right), 124.3\left(\mathrm{CH}_{\mathrm{Ar}}\right), 114.0\left(2 \times \mathrm{CH}_{\mathrm{Ar}}\right)$, $83.4(\mathrm{CH}-\mathrm{O}), 80.9(\mathrm{CH}-\mathrm{O}), 79.9(\mathrm{C}), 55.4\left(\mathrm{OCH}_{3}\right), 44.5\left(\mathrm{CH}_{2}\right), 26.4\left(\mathrm{CH}_{3}\right), 15.8\left(\mathrm{CH}_{3}\right)$.

HRMS (ESI) m/z: [M+H'] Calcd for $\mathrm{C}_{19} \mathrm{H}_{21} \mathrm{~N}_{2} \mathrm{O}_{3} \mathrm{~S}$ 357.1267, found 357.1265.

$\left(5 R^{*}, 6 R^{*}, 8 R^{*}\right)-2,3-$ dimethyl-8-(4-methoxyphenyl)-6-(thiophen-2-yl)-7-oxa-1,3-diazaspiro[4.4]non-1en-4-one 3'ai

Yield $35 \mathrm{mg}$ (0.10 mmol, 33\%). Yellowish oil; $\mathrm{R}_{f}=0.34$ (EtOAc, UV/vanillin viz.).

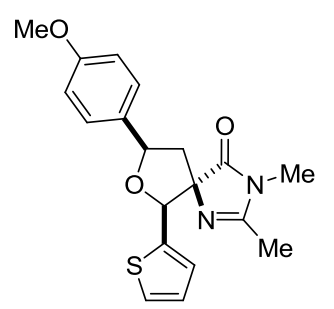

${ }^{1} \mathrm{H} \mathrm{NMR}\left(700 \mathrm{MHz}, 303 \mathrm{~K}, \mathrm{CDCl}_{3}\right): \delta=7.58(\mathrm{~d}, J=8.6 \mathrm{~Hz}, 2 \mathrm{H}), 7.25(\mathrm{~d}, J=5.0 \mathrm{~Hz}, 1 \mathrm{H})$, 6.94-6.84 (m, 4H), $5.37(\mathrm{~s}, 1 \mathrm{H}), 5.24(\mathrm{t}, J=7.9 \mathrm{~Hz}, 1 \mathrm{H}), 3.80(\mathrm{~s}, 3 \mathrm{H}), 2.98(\mathrm{~s}, 3 \mathrm{H}), 2.85$ (dd, $J=13.3,8.4 \mathrm{~Hz}, 1 \mathrm{H}$ ), $2.27(\mathrm{dd}, J=13.3,7.3 \mathrm{~Hz}, 1 \mathrm{H}), 2.05(\mathrm{~s}, 3 \mathrm{H})$.

${ }^{13} \mathrm{C}$ NMR (175 MHz, $303 \mathrm{~K}, \mathrm{CDCl}_{3}$ ): $\delta=182.4$ (C=0), 160.4 (C), 159.5 (C), 137.9 (C), $133.4(\mathrm{C}), 128.3\left(2 \times \mathrm{CH}_{\mathrm{Ar}}\right), 126.3\left(\mathrm{CH}_{\mathrm{Ar}}\right), 126.0\left(\mathrm{CH}_{\mathrm{Ar}}\right), 125.8\left(\mathrm{CH}_{\mathrm{Ar}}\right), 113.9\left(2 \times \mathrm{CH}_{\mathrm{Ar}}\right)$, $85.1(\mathrm{CH}-\mathrm{O}), 80.4(\mathrm{CH}-\mathrm{O}), 78.9(\mathrm{C}), 55.4\left(\mathrm{OCH}_{3}\right), 45.0\left(\mathrm{CH}_{2}\right), 26.8\left(\mathrm{CH}_{3}\right), 15.3\left(\mathrm{CH}_{3}\right)$.

HRMS (ESI) m/z: [M+H'] Calcd for $\mathrm{C}_{19} \mathrm{H}_{21} \mathrm{~N}_{2} \mathrm{O}_{3} \mathrm{~S}$ 357.1267, found 357.1268. 
$\left(5 S^{*}, 6 R^{*}, 8 R^{*}\right)$-2,3-dimethyl-8-(4-methoxyphenyl)-6-(4-methoxystyryl)-7-oxa-1,3-diazaspiro[4.4]non-1en-4-one 3aj

Yield $39 \mathrm{mg}(0.10 \mathrm{mmol}, 32 \%)$. Yellowish crystals, $\mathrm{mp}=107-108^{\circ} \mathrm{C} ; \mathrm{R}_{f}=0.12$ (EtOAc, UV/vanillin $/ \mathrm{KMnO}_{4}$ viz.).

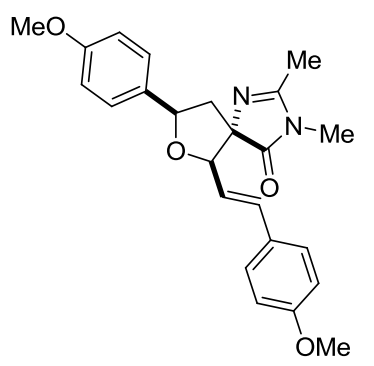

${ }^{1} \mathrm{H} \mathrm{NMR}\left(700 \mathrm{MHz}, 303 \mathrm{~K}, \mathrm{CDCl}_{3}\right): \delta=7.50(\mathrm{~d}, J=8.6 \mathrm{~Hz}, 2 \mathrm{H}), 7.30(\mathrm{~d}, J=8.7 \mathrm{~Hz}$, $2 \mathrm{H}), 6.90(\mathrm{~d}, J=8.7 \mathrm{~Hz}, 2 \mathrm{H}), 6.82(\mathrm{~d}, J=8.6 \mathrm{~Hz}, 2 \mathrm{H}), 6.53(\mathrm{~d}, J=15.8 \mathrm{~Hz}, 1 \mathrm{H}), 6.20$ $(\mathrm{dd}, J=15.8,8.4 \mathrm{~Hz}, 1 \mathrm{H}), 5.16(\mathrm{dd}, J=9.7,6.3 \mathrm{~Hz}, 1 \mathrm{H}), 4.60(\mathrm{~d}, J=8.5 \mathrm{~Hz}, 1 \mathrm{H})$, $3.80(\mathrm{~s}, 3 \mathrm{H}), 3.79(\mathrm{~s}, 3 \mathrm{H}), 2.97(\mathrm{~s}, 3 \mathrm{H}), 2.50(\mathrm{dd}, J=13.1,6.3 \mathrm{~Hz}, 1 \mathrm{H}), 2.40$ (dd, $J=$ $13.1,9.7 \mathrm{~Hz}, 1 \mathrm{H}), 2.21(\mathrm{~s}, 3 \mathrm{H})$.

$\left.{ }^{13} \mathrm{C} \mathrm{NMR} \mathrm{(175} \mathrm{MHz,} 303 \mathrm{~K}, \mathrm{CDCl}_{3}\right): \delta=182.5$ (C=O), 161.5 (C), $159.6^{8}$ (C), 159.6 (C), $133.7(\mathrm{CH}), 132.5(\mathrm{C}), 129.3(\mathrm{C}), 128.4\left(2 \times \mathrm{CH}_{\mathrm{Ar}}\right), 128.2\left(2 \times \mathrm{CH}_{\mathrm{Ar}}\right), 122.1(\mathrm{CH})$, $114.0^{6}\left(2 \times \mathrm{CH}_{\mathrm{Ar}}\right), 114.0^{1}\left(2 \times \mathrm{CH}_{\mathrm{Ar}}\right), 86.4(\mathrm{CH}-\mathrm{O}), 80.9(\mathrm{CH}-\mathrm{O}), 79.6(\mathrm{C}), 55.4^{2}\left(\mathrm{OCH}_{3}\right), 55.4^{1}\left(\mathrm{OCH}_{3}\right), 45.4\left(\mathrm{CH}_{2}\right)$, $26.7\left(\mathrm{CH}_{3}\right), 15.8\left(\mathrm{CH}_{3}\right)$.

HRMS (ESI) m/z: [M+H'] Calcd for $\mathrm{C}_{24} \mathrm{H}_{27} \mathrm{~N}_{2} \mathrm{O}_{4} 407.1965$, found 407.1963.

$\left(5 R^{*}, 6 R^{*}, 8 R^{*}\right)$-2,3-dimethyl-8-(4-methoxyphenyl)-6-(4-methoxystyryl)-7-oxa-1,3-diazaspiro[4.4]non-1en-4-one 3'aj

Yield $63 \mathrm{mg}(0.16 \mathrm{mmol}, 52 \%)$. Yellowish crystals, $\mathrm{mp}=87-88{ }^{\circ} \mathrm{C}\left(\mathrm{Et}_{2} \mathrm{O}\right) ; \mathrm{R}_{f}=0.27$ (EtOAc, UV/vanillin/ $\mathrm{KMnO}_{4}$ viz.).

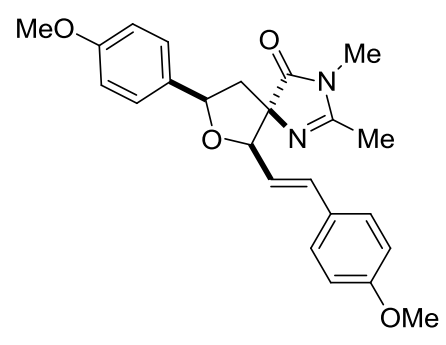

${ }^{1} \mathrm{H} \mathrm{NMR}\left(700 \mathrm{MHz}, 303 \mathrm{~K}, \mathrm{CDCl}_{3}\right): \delta=7.48(\mathrm{~d}, J=8.3 \mathrm{~Hz}, 2 \mathrm{H}), 7.28(\mathrm{~d}, J=8.4$ $\mathrm{Hz}, 2 \mathrm{H}), 6.89(\mathrm{~d}, J=8.3 \mathrm{~Hz}, 2 \mathrm{H}), 6.82(\mathrm{~d}, J=8.4 \mathrm{~Hz}, 2 \mathrm{H}), 6.47(\mathrm{~d}, J=15.9 \mathrm{~Hz}$, $1 \mathrm{H}), 6.11(\mathrm{dd}, J=15.9,8.2 \mathrm{~Hz}, 1 \mathrm{H}), 5.16(\mathrm{t}, J=7.9 \mathrm{~Hz}, 1 \mathrm{H}), 4.61(\mathrm{~d}, J=8.2$ $\mathrm{Hz}, 1 \mathrm{H}), 3.80(\mathrm{~s}, 3 \mathrm{H}), 3.78(\mathrm{~s}, 3 \mathrm{H}), 3.03(\mathrm{~s}, 3 \mathrm{H}), 2.74(\mathrm{dd}, J=13.2,7.9 \mathrm{~Hz}, 1 \mathrm{H})$, $2.21(\mathrm{dd}, J=13.2,7.9 \mathrm{~Hz}, 1 \mathrm{H}), 2.19(\mathrm{~s}, 3 \mathrm{H})$.

$159.5(\mathrm{C}), 133.6(\mathrm{CH}), 133.3(\mathrm{C}), 129.2(\mathrm{C}), 129.2\left(2 \times \mathrm{CH}_{\mathrm{Ar}}\right), 128.1\left(2 \times \mathrm{CH}_{\mathrm{Ar}}\right), 121.7(\mathrm{CH}), 114.0\left(2 \times \mathrm{CH}_{\mathrm{Ar}}\right), 113.9$ $\left(2 \times \mathrm{CH}_{\mathrm{Ar}}\right), 88.4(\mathrm{CH}-\mathrm{O}), 80.7(\mathrm{CH}-\mathrm{O}), 79.9(\mathrm{C}), 55.4^{0}\left(\mathrm{OCH}_{3}\right), 55.3^{8}\left(\mathrm{OCH}_{3}\right), 45.3\left(\mathrm{CH}_{2}\right), 26.8\left(\mathrm{CH}_{3}\right), 15.6\left(\mathrm{CH}_{3}\right)$.

HRMS (ESI) m/z: [M+H'] Calcd for $\mathrm{C}_{24} \mathrm{H}_{27} \mathrm{~N}_{2} \mathrm{O}_{4} 407.1965$, found 407.1968. 
$\left(5 S^{*}, 6 R^{*}, 8 R^{*}\right)-6$-cyclohexyl-2,3-dimethyl-8-(4-methoxyphenyl)-7-oxa-1,3-diazaspiro[4.4]non-1-en-4one 3ak

Yield $48 \mathrm{mg}(0.13 \mathrm{mmol}, 45 \%)$. Colorless crystals, $\mathrm{mp}=101-102{ }^{\circ} \mathrm{C}$ (hexane); $\mathrm{R}_{f}=0.12$ (EtOAc, UV/vanillin viz.).

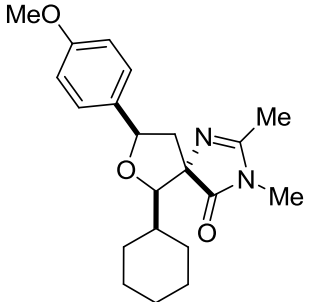

${ }^{1} \mathrm{H} \mathrm{NMR}\left(700 \mathrm{MHz}, 303 \mathrm{~K}, \mathrm{CDCl}_{3}\right): \delta=7.39(\mathrm{~d}, J=8.3 \mathrm{~Hz}, 2 \mathrm{H}), 6.88(\mathrm{~d}, J=8.3 \mathrm{~Hz}, 2 \mathrm{H})$, $5.01(\mathrm{dd}, J=10.3,5.5 \mathrm{~Hz}, 1 \mathrm{H}), 3.81(\mathrm{~d}, J=9.8 \mathrm{~Hz}, 1 \mathrm{H}), 3.79(\mathrm{~s}, 3 \mathrm{H}), 3.08(\mathrm{~s}, 3 \mathrm{H}), 2.29$ (dd, $J=12.7,5.5 \mathrm{~Hz}, 1 \mathrm{H}), 2.21(\mathrm{~s}, 3 \mathrm{H}), 2.19(\mathrm{dd}, J=12.7,10.3 \mathrm{~Hz}, 1 \mathrm{H}), 2.11(\mathrm{br} \mathrm{d}, J=$ $13.6 \mathrm{~Hz}, 1 \mathrm{H}), 1.96$ (qt, $J=10.9,3.5 \mathrm{~Hz}, 1 \mathrm{H}), 1.71-1.65(\mathrm{~m}, 1 \mathrm{H}), 1.62-1.54(\mathrm{~m}, 2 \mathrm{H})$, $1.31-1.16(\mathrm{~m}, 2 \mathrm{H}), 1.12(\mathrm{ddt}, J=16.0,12.6,6.3 \mathrm{~Hz}, 1 \mathrm{H}), 1.05-0.93(\mathrm{~m}, 2 \mathrm{H}), 0.84$ (qd, $J=12.1,3.3 \mathrm{~Hz}, 1 \mathrm{H})$.

${ }^{13} \mathrm{C} \mathrm{NMR}\left(175 \mathrm{MHz}, 303 \mathrm{~K}, \mathrm{CDCl}_{3}\right): \delta=183.2,160.1,159.5,132.7,128.1(2 \times), 114.0(2 \times), 89.3,79.7,78.1$, $55.4,48.4,38.2,30.6,28.7,26.7,26.5,25.9,25.6,15.9$.

HRMS (ESI) m/z: [M+H'] Calcd for $\mathrm{C}_{21} \mathrm{H}_{29} \mathrm{~N}_{2} \mathrm{O}_{3} 357.2173$, found 357.2171.

$\left(5 R^{*}, 6 R^{*}, 8 R^{*}\right)-6$-cyclohexyl-2,3-dimethyl-8-(4-methoxyphenyl)-7-oxa-1,3-diazaspiro[4.4]non-1-en-4one 3'ak

Yield $8 \mathrm{mg}$ (0.02 mmol, 7\%). Yellowish oil; $\mathrm{R}_{f}=0.35$ (EtOAc, UV/vanillin viz.).

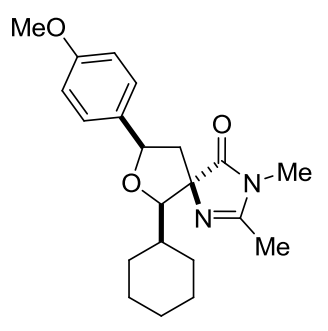

${ }^{1} \mathrm{H} \mathrm{NMR}\left(700 \mathrm{MHz}, 303 \mathrm{~K}, \mathrm{CDCl}_{3}\right): \delta=7.41(\mathrm{~d}, J=8.6 \mathrm{~Hz}, 2 \mathrm{H}), 6.87(\mathrm{~d}, J=8.6 \mathrm{~Hz}, 2 \mathrm{H})$, $4.99(\mathrm{t}, J=7.9 \mathrm{~Hz}, 1 \mathrm{H}), 3.79(\mathrm{~s}, 3 \mathrm{H}), 3.76(\mathrm{~d}, J=9.1 \mathrm{~Hz}, 1 \mathrm{H}), 3.06(\mathrm{~s}, 3 \mathrm{H}), 2.60(\mathrm{dd}, J$ = 13.1, $7.7 \mathrm{~Hz}, 1 \mathrm{H}), 2.25(\mathrm{~s}, 3 \mathrm{H}), 2.08(\mathrm{br} \mathrm{d}, J=13.3 \mathrm{~Hz}, 1 \mathrm{H}), 2.02(\mathrm{dd}, J=13.1,8.2$ $\mathrm{Hz}, 1 \mathrm{H}), 1.82-1.74(\mathrm{~m}, 1 \mathrm{H}), 1.70-1.65(\mathrm{~m}, 1 \mathrm{H}), 1.62-1.57(\mathrm{~m}, 2 \mathrm{H}), 1.24-1.09(\mathrm{~m}$, $3 \mathrm{H}), 1.04(q d, J=12.6,3.6 \mathrm{~Hz}, 1 \mathrm{H}), 0.94-0.90(\mathrm{~m}, 1 \mathrm{H}), 0.83(q d, J=12.1,3.2 \mathrm{~Hz}, 1 \mathrm{H})$.

${ }^{13} \mathrm{C} \mathrm{NMR}\left(175 \mathrm{MHz}, 303 \mathrm{~K}, \mathrm{CDCl}_{3}\right): \delta=184.1,160.0,159.4,133.8,128.1(2 \times), 113.9$ (2x), 90.1, 79.5, 77.8, 55.4, 47.6, 38.3, 30.5, 28.6, 26.9, 26.5, 25.9, 25.7, 15.3.

HRMS (ESI) m/z: [M+H'] Calcd for $\mathrm{C}_{21} \mathrm{H}_{29} \mathrm{~N}_{2} \mathrm{O}_{3}$ 357.2173, found 357.2177. 
$\left(5 S^{*}, 6 R^{*}, 8 R^{*}\right)-2,3-D i m e t h y l-8-(2-m e t h o x y p h e n y l)-6-(3,4,5-t r i m e t h o x y p h e n y l)-7-o x a-1,3-$ diazaspiro[4.4]non-1-en-4-one 3ba

Yield $43 \mathrm{mg}(0.10 \mathrm{mmol}, 33 \%)$. Colorless prysms; $\mathrm{mp}=80-82{ }^{\circ} \mathrm{C}\left(\mathrm{Et}_{2} \mathrm{O}\right) ; \mathrm{R}_{f}=0.08$ (EtOAc, UV/vanillin viz.).

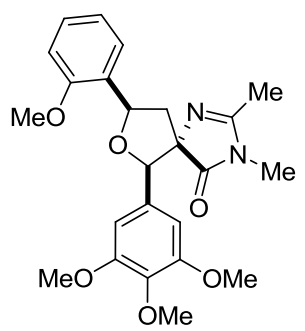

${ }^{1} \mathrm{H} \mathrm{NMR}\left(700 \mathrm{MHz}, 303 \mathrm{~K}, \mathrm{CDCl}_{3}\right): \delta=7.94(\mathrm{dd}, J=7.6,1.8 \mathrm{~Hz}, 1 \mathrm{H}), 7.28(\mathrm{td}, J=7.8$, $1.8 \mathrm{~Hz}, 1 \mathrm{H}), 7.07(\mathrm{td}, J=7.4 .1 .0 \mathrm{~Hz}, 1 \mathrm{H}), 6.88(\mathrm{dd}, J=8.2,1.0 \mathrm{~Hz}, 1 \mathrm{H}), 6.64(\mathrm{~s}, 2 \mathrm{H})$, $5.65(\mathrm{dd}, J=8.7,7.4 \mathrm{~Hz}, 1 \mathrm{H}), 5.06(\mathrm{~s}, 1 \mathrm{H}), 3.85(\mathrm{~s}, 6 \mathrm{H}), 3.83(\mathrm{~s}, 3 \mathrm{H}), 3.82(\mathrm{~s}, 3 \mathrm{H}), 2.81$ $(\mathrm{dd}, J=13.2,7.4 \mathrm{~Hz}, 1 \mathrm{H}), 2.70(\mathrm{~s}, 3 \mathrm{H}), 2.25(\mathrm{dd}, J=13.2,8.7 \mathrm{~Hz}, 1 \mathrm{H}), 2.18(\mathrm{~s}, 3 \mathrm{H})$.

${ }^{13} \mathrm{C} \mathrm{NMR}\left(75 \mathrm{MHz}, 303 \mathrm{~K}, \mathrm{CDCl}_{3}\right): \delta=182.1(\mathrm{C}=\mathrm{O}), 161.8(\mathrm{C}), 156.3(\mathrm{C}), 152.8$ (2×O$\left.\mathrm{C}_{\mathrm{Ar}}\right), 137.8(\mathrm{C}), 131.7(\mathrm{C}), 129.6(\mathrm{C}), 128.6\left(\mathrm{CH}_{\mathrm{Ar}}\right), 127.1\left(\mathrm{CH}_{\mathrm{Ar}}\right), 121.1\left(\mathrm{CH}_{\mathrm{Ar}}\right), 110.2$

$\left(\mathrm{CH}_{\mathrm{Ar}}\right), 103.5\left(2 \times \mathrm{CH}_{\mathrm{Ar}}\right), 86.1(\mathrm{CH}-\mathrm{O}), 80.2(\mathrm{C}), 75.0(\mathrm{CH}-\mathrm{O}), 61.0\left(\mathrm{OCH}_{3}\right), 56.3\left(2 \times \mathrm{OCH}_{3}\right), 55.5\left(\mathrm{OCH}_{3}\right), 43.3$ $\left(\mathrm{CH}_{2}\right), 26.2\left(\mathrm{CH}_{3}\right), 15.7\left(\mathrm{CH}_{3}\right)$.

HRMS (ESI) m/z: [M+H'] Calcd for $\mathrm{C}_{24} \mathrm{H}_{29} \mathrm{~N}_{2} \mathrm{O}_{6} 441.2020$, found 441.2018.

$\left(5 R^{*}, 6 R^{*}, 8 R^{*}\right)-2,3-D i m e t h y l-8-(2-m e t h o x y p h e n y l)-6-(3,4,5-t r i m e t h o x y p h e n y l)-7-o x a-1,3-$ diazaspiro[4.4]non-1-en-4-one 3 'ba

Yield $35 \mathrm{mg}$ (0.08 mmol, 27\%). Colorless oil; $\mathrm{R}_{f}=0.25$ (EtOAc, UV/vanillin viz.).

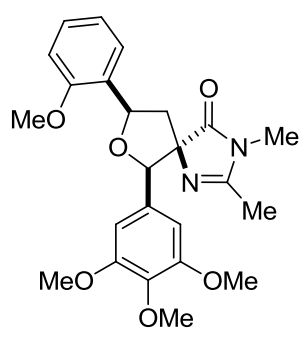

${ }^{1} \mathrm{H} \mathrm{NMR}\left(700 \mathrm{MHz}, 303 \mathrm{~K}, \mathrm{CDCl}_{3}\right): \delta=7.86(\mathrm{dd}, J=7.5,1.8 \mathrm{~Hz}, 1 \mathrm{H}), 7.26(\mathrm{td}, J=7.8$, $1.8 \mathrm{~Hz}, 1 \mathrm{H}), 7.03(\mathrm{t}, J=7.4 \mathrm{~Hz}, 1 \mathrm{H}), 6.87(\mathrm{~d}, J=8.1 \mathrm{~Hz}, 1 \mathrm{H}), 6.56(\mathrm{~s}, 2 \mathrm{H}), 5.61(\mathrm{t}, J=$ $8.0 \mathrm{~Hz}, 1 \mathrm{H}), 5.05(\mathrm{~s}, 1 \mathrm{H}), 3.84(\mathrm{~s}, 6 \mathrm{H}), 3.83(\mathrm{~s}, 3 \mathrm{H}), 3.82(\mathrm{~s}, 3 \mathrm{H}), 3.01(\mathrm{dd}, \mathrm{J}=13.3,8.4$ $\mathrm{Hz}, 1 \mathrm{H}), 2.98(\mathrm{~s}, 3 \mathrm{H}), 2.09(\mathrm{dd}, J=13.3,7.6 \mathrm{~Hz}, 1 \mathrm{H}), 1.92(\mathrm{~s}, 3 \mathrm{H})$.

${ }^{13} \mathrm{C}$ NMR $\left(75 \mathrm{MHz}, 303 \mathrm{~K}, \mathrm{CDCl}_{3}\right): \delta=182.6$ (C=O), 159.9 (C), 156.1 (C), 152.8 (2×O$\left.\mathrm{C}_{\mathrm{Ar}}\right), 137.6(\mathrm{C}), 131.3(\mathrm{C}), 130.1(\mathrm{C}), 128.4\left(\mathrm{CH}_{\mathrm{Ar}}\right), 126.4\left(\mathrm{CH}_{\mathrm{Ar}}\right), 120.9\left(\mathrm{CH}_{\mathrm{Ar}}\right), 110.1$ $\left(\mathrm{CH}_{\mathrm{Ar}}\right), 103.8\left(2 \times \mathrm{CH}_{\mathrm{Ar}}\right), 88.6(\mathrm{CH}-\mathrm{O}), 79.2(\mathrm{C}), 75.1(\mathrm{CH}-\mathrm{O}), 61.0\left(\mathrm{OCH}_{3}\right), 56.8\left(2 \times \mathrm{OCH}_{3}\right), 55.4\left(\mathrm{OCH}_{3}\right), 44.0$ $\left(\mathrm{CH}_{2}\right), 26.8\left(\mathrm{CH}_{3}\right), 15.2\left(\mathrm{CH}_{3}\right)$.

HRMS (ESI) m/z: [M+H'] Calcd for $\mathrm{C}_{24} \mathrm{H}_{29} \mathrm{~N}_{2} \mathrm{O}_{6} 441.2020$, found 441.2022. 
Yield $53 \mathrm{mg}(0.11 \mathrm{mmol}, 36 \%)$. Colorless crystals; $\mathrm{mp}=131-132{ }^{\circ} \mathrm{C}\left(\mathrm{Et}_{2} \mathrm{O}\right) ; \mathrm{R}_{f}=0.08(\mathrm{EtOAc} / \mathrm{iPrOH}=9 / 1$, UV/vanillin viz.).<smiles>COc1cc(C(=O)C2C(c3cc(OC)c(OC)c(OC)c3)N=C(C)N2C)cc(OC)c1OC</smiles>

${ }^{1} \mathrm{H} \mathrm{NMR}\left(700 \mathrm{MHz}, 303 \mathrm{~K}, \mathrm{CDCl}_{3}\right): \delta=7.02(\mathrm{~s}, 2 \mathrm{H}), 6.62(\mathrm{~s}, 2 \mathrm{H}), 5.21(\mathrm{t}, J=8.0 \mathrm{~Hz}$, $1 \mathrm{H}), 5.06(\mathrm{~s}, 1 \mathrm{H}), 3.93(\mathrm{~s}, 6 \mathrm{H}), 3.85(\mathrm{~s}, 3 \mathrm{H}), 3.82(\mathrm{~s}, 6 \mathrm{H}), 3.81(\mathrm{~s}, 3 \mathrm{H}), 2.75(\mathrm{dd}, J=$ $13.4,7.9 \mathrm{~Hz}, 1 \mathrm{H}), 2.71(\mathrm{~s}, 3 \mathrm{H}), 2.43(\mathrm{dd}, J=13.4,8.2 \mathrm{~Hz}, 1 \mathrm{H}), 2.18(\mathrm{~s}, 3 \mathrm{H})$.

${ }^{13} \mathrm{C}$ NMR (175 MHz, $\left.303 \mathrm{~K}, \mathrm{CDCl}_{3}\right): \delta=182.2(\mathrm{C}=0), 162.1(\mathrm{C}), 153.5\left(2 \times \mathrm{O}-\mathrm{C}_{\mathrm{Ar}}\right)$, $152.8\left(2 \times 0-\mathrm{C}_{\mathrm{Ar}}\right), 137.8^{9}$ (C), $137.8^{4}(\mathrm{C}), 136.8(\mathrm{C}), 131.1$ (C), $104.4\left(2 \times \mathrm{CH}_{\mathrm{Ar}}\right), 103.2$ $\left(2 \times \mathrm{CH}_{\mathrm{Ar}}\right), 86.3(\mathrm{CH}-\mathrm{O}), 80.9(\mathrm{CH}-\mathrm{O}), 79.9(\mathrm{C}), 61.0\left(2 \times \mathrm{OCH}_{3}\right), 56.3\left(2 \times \mathrm{OCH}_{3}\right), 56.2$ $\left(2 \times \mathrm{OCH}_{3}\right), 44.0\left(\mathrm{CH}_{2}\right), 26.3\left(\mathrm{CH}_{3}\right), 15.7\left(\mathrm{CH}_{3}\right)$.

HRMS (ESI) m/z: [M+H'] Calcd for $\mathrm{C}_{26} \mathrm{H}_{33} \mathrm{~N}_{2} \mathrm{O}_{8}$ 501.2231, found 501.2232.

$\left(5 R^{*}, 6 R^{*}, 8 R^{*}\right)-6,8-B i s(3,4,5-t r i m e t h o x y p h e n y l)-2,3-d i m e t h y l-7-o x a-1,3-d i a z a s p i r o[4.4]$ non-1-en-4-one $3^{\prime} \mathrm{ca}$

Yield $41 \mathrm{mg}$ (0.08 mmol, 27\%). Amorphous solid; $\mathrm{R}_{f}=0.22$ (EtOAc/iPrOH = 9/1, UV/vanillin viz.).

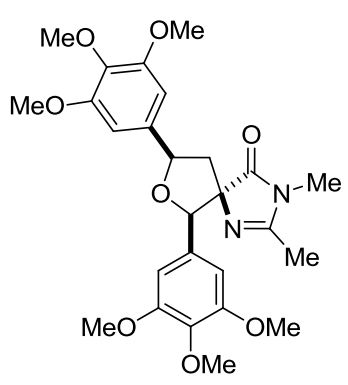

${ }^{1} \mathrm{H} \mathrm{NMR}\left(700 \mathrm{MHz}, 303 \mathrm{~K}, \mathrm{CDCl}_{3}\right): \delta=6.95(\mathrm{~s}, 2 \mathrm{H}), 6.52(\mathrm{~s}, 2 \mathrm{H}), 5.25(\mathrm{dd}, J=8.7$, $6.6 \mathrm{~Hz}, 1 \mathrm{H}), 5.07(\mathrm{~s}, 1 \mathrm{H}), 3.90(\mathrm{~s}, 6 \mathrm{H}), 3.86(\mathrm{~s}, 3 \mathrm{H}), 3.81(\mathrm{~s}, 3 \mathrm{H}), 3.80(\mathrm{~s}, 6 \mathrm{H}), 2.99$ $(\mathrm{s}, 3 \mathrm{H}), 2.91(\mathrm{dd}, J=13.2,8.7 \mathrm{~Hz}, 1 \mathrm{H}), 2.25(\mathrm{dd}, J=13.2,6.6 \mathrm{~Hz}, 1 \mathrm{H}), 1.96(\mathrm{~s}, 3 \mathrm{H})$.

${ }^{13} \mathrm{C} \mathrm{NMR}\left(75 \mathrm{MHz}, 303 \mathrm{~K}, \mathrm{CDCl}_{3}\right): \delta=182.7(\mathrm{C}=0), 159.5(\mathrm{C}), 153.4\left(2 \times \mathrm{O}-\mathrm{C}_{\mathrm{Ar}}\right), 152.8$ $\left(2 \times 0-\mathrm{C}_{\mathrm{Ar}}\right), 137.6^{4}$ (C), $137.6^{1}$ (C), 137.4 (C), 131.2 (C), $104.2\left(2 \times \mathrm{CH}_{\mathrm{Ar}}\right), 103.7$ $\left(2 \times \mathrm{CH}_{\mathrm{Ar}}\right), 88.9(\mathrm{CH}-\mathrm{O}), 80.8(\mathrm{CH}-\mathrm{O}), 79.1(\mathrm{C}), 61.0\left(2 \times \mathrm{OCH}_{3}\right), 56.2^{1}\left(2 \times \mathrm{OCH}_{3}\right), 56.1^{7}$ $\left(2 \times \mathrm{OCH}_{3}\right), 44.8\left(\mathrm{CH}_{2}\right), 26.9\left(\mathrm{CH}_{3}\right), 15.4\left(\mathrm{CH}_{3}\right)$.

HRMS (ESI) m/z: [M+H'] Calcd for $\mathrm{C}_{26} \mathrm{H}_{33} \mathrm{~N}_{2} \mathrm{O}_{8}$ 501.2231, found 501.2235. 
$\left(5 S^{*}, 6 R^{*}, 8 R^{*}\right)-8-(3,4-D i m e t h o x y p h e n y l)-2,3-d i m e t h y l-6-(3,4,5-t r i m e t h o x y p h e n y l)-7-o x a-1,3-$ diazaspiro[4.4]non-1-en-4-one 3da

Yield $62 \mathrm{mg}(0.13 \mathrm{mmol}, 44 \%)$. Colorless crystals; $\mathrm{mp}=137-139{ }^{\circ} \mathrm{C}\left(\mathrm{Et}_{2} \mathrm{O}\right) ; \mathrm{R}_{f}=0.09(\mathrm{EtOAc} / \mathrm{iPrOH}=9 / 1$, UV/vanillin viz.).

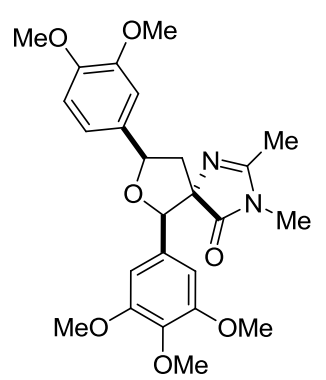

${ }^{1} \mathrm{H}$ NMR $\left(700 \mathrm{MHz}, 303 \mathrm{~K}, \mathrm{CDCl}_{3}\right): \delta=7.49(\mathrm{~d}, J=1.9 \mathrm{~Hz}, 1 \mathrm{H}), 7.13(\mathrm{dd}, J=8.1,1.9$ $\mathrm{Hz}, 1 \mathrm{H}), 6.88(\mathrm{~d}, J=8.1 \mathrm{~Hz}, 1 \mathrm{H}), 6.61(\mathrm{~s}, 2 \mathrm{H}), 5.23(\mathrm{t}, J=8.0 \mathrm{~Hz}, 1 \mathrm{H}), 5.05(\mathrm{~s}, 1 \mathrm{H}), 3.98$ $(\mathrm{s}, 3 \mathrm{H}), 3.90(\mathrm{~s}, 3 \mathrm{H}), 3.82(\mathrm{~s}, 6 \mathrm{H}), 3.81(\mathrm{~s}, 3 \mathrm{H}), 2.72(\mathrm{dd}, J=13.3,7.8 \mathrm{~Hz}, 1 \mathrm{H}), 2.71(\mathrm{~s}$, $3 \mathrm{H}), 2.44(\mathrm{dd}, J=13.3,8.3 \mathrm{~Hz}, 1 \mathrm{H}), 2.18(\mathrm{~s}, 3 \mathrm{H})$.

${ }^{13} \mathrm{C}$ NMR (175 MHz, $\left.303 \mathrm{~K}, \mathrm{CDCl}_{3}\right): \delta=182.3(\mathrm{C}=\mathrm{O}), 162.0(\mathrm{C}), 152.8\left(2 \times \mathrm{O}-\mathrm{C}_{\mathrm{Ar}}\right), 149.4$ (C), 149.1 (C), 137.8 (C), 133.7 (C), $131.2(\mathrm{C}), 119.6\left(\mathrm{CH}_{\mathrm{Ar}}\right), 110.9\left(\mathrm{CH}_{\mathrm{Ar}}\right), 110.7\left(\mathrm{CH}_{\mathrm{Ar}}\right)$, $103.3\left(2 \times \mathrm{CH}_{\mathrm{Ar}}\right), 86.3(\mathrm{CH}-\mathrm{O}), 80.7(\mathrm{CH}-\mathrm{O}), 80.0(\mathrm{C}), 61.0\left(\mathrm{OCH}_{3}\right), 56.2\left(2 \times \mathrm{OCH}_{3}\right), 56.0^{9}$ $\left(\mathrm{OCH}_{3}\right), 56.0^{1}\left(\mathrm{OCH}_{3}\right), 44.1\left(\mathrm{CH}_{2}\right), 26.3\left(\mathrm{CH}_{3}\right), 15.9\left(\mathrm{CH}_{3}\right)$.

HRMS (ESI) m/z: [M+H'] Calcd for $\mathrm{C}_{25} \mathrm{H}_{31} \mathrm{~N}_{2} \mathrm{O}_{7} 471.2126$, found 471.2126.

$\left(5 R^{*}, 6 R^{*}, 8 R^{*}\right)-8-(3,4-D i m e t h o x y p h e n y l)-2,3-d i m e t h y l-6-(3,4,5-t r i m e t h o x y p h e n y l)-7-o x a-1,3-$ diazaspiro[4.4]non-1-en-4-one 3 'da

Yield $51 \mathrm{mg}(0.11 \mathrm{mmol}, 36 \%)$. Colorless crystals; $\mathrm{mp}=149-150^{\circ} \mathrm{C}$ (hexane/EtOAc); $\mathrm{R}_{f}=0.19$ (EtOAc/iPrOH = 9/1, UV/vanillin viz.).

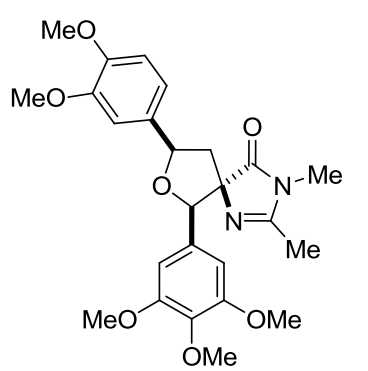

${ }^{1} \mathrm{H}$ NMR $\left(700 \mathrm{MHz}, 303 \mathrm{~K}, \mathrm{CDCl}_{3}\right): \delta=7.38(\mathrm{~d}, J=1.9 \mathrm{~Hz}, 1 \mathrm{H}), 7.11(\mathrm{dd}, J=8.2,1.9$ $\mathrm{Hz}, 1 \mathrm{H}), 6.87(\mathrm{~d}, J=8.2 \mathrm{~Hz}, 1 \mathrm{H}), 6.51(\mathrm{~s}, 2 \mathrm{H}), 5.26(\mathrm{dd}, J=8.6,6.8 \mathrm{~Hz}, 1 \mathrm{H}), 5.07(\mathrm{~s}$, $1 \mathrm{H}), 3.93(\mathrm{~s}, 3 \mathrm{H}), 3.89(\mathrm{~s}, 3 \mathrm{H}), 3.81(\mathrm{~s}, 3 \mathrm{H}), 3.80(\mathrm{~s}, 6 \mathrm{H}), 3.00(\mathrm{~s}, 3 \mathrm{H}), 2.90(\mathrm{dd}, J=$ $13.2,8.6 \mathrm{~Hz}, 1 \mathrm{H}), 2.25(\mathrm{dd}, J=13.2,6.8 \mathrm{~Hz}, 1 \mathrm{H}), 1.96(\mathrm{~s}, 3 \mathrm{H})$.

${ }^{13} \mathrm{C} \mathrm{NMR}\left(75 \mathrm{MHz}, 303 \mathrm{~K}, \mathrm{DMSO}-\boldsymbol{d}_{6}\right): \delta=181.8(\mathrm{C}=\mathrm{O}), 160.0(\mathrm{C}), 152.2\left(2 \times \mathrm{O}-\mathrm{C}_{\mathrm{Ar}}\right)$, 148.7 (C), 148.3 (C), 136.8 (C), 134.8 (C), 131.7 (C), $118.9\left(\mathrm{CH}_{\mathrm{Ar}}\right), 111.4\left(\mathrm{CH}_{\mathrm{Ar}}\right)$, $110.8\left(\mathrm{CH}_{\mathrm{Ar}}\right), 103.5\left(2 \times \mathrm{CH}_{\mathrm{Ar}}\right), 87.2(\mathrm{CH}-\mathrm{O}), 79.3(\mathrm{CH}-\mathrm{O}), 78.3(\mathrm{C}), 60.0\left(\mathrm{OCH}_{3}\right), 55.7$ $\left(2 \times \mathrm{OCH}_{3}\right), 55.5\left(\mathrm{OCH}_{3}\right), 55.3\left(\mathrm{OCH}_{3}\right), 44.6\left(\mathrm{CH}_{2}\right), 26.4\left(\mathrm{CH}_{3}\right), 14.9\left(\mathrm{CH}_{3}\right)$.

HRMS (ESI) m/z: [M+H'] Calcd for $\mathrm{C}_{25} \mathrm{H}_{31} \mathrm{~N}_{2} \mathrm{O}_{7}$ 471.2126, found 471.2125. 


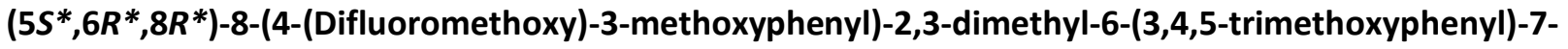
oxa-1,3-diazaspiro[4.4]non-1-en-4-one 3ea

Yield $52 \mathrm{mg}(0.10 \mathrm{mmol}, 34 \%)$. Colorless crystals, $\mathrm{mp}=128-130{ }^{\circ} \mathrm{C}\left(\mathrm{Et}_{2} \mathrm{O}\right) ; \mathrm{R}_{f}=0.02(\mathrm{EtOAc}, \mathrm{UV} / \mathrm{vanillin}$ viz.).

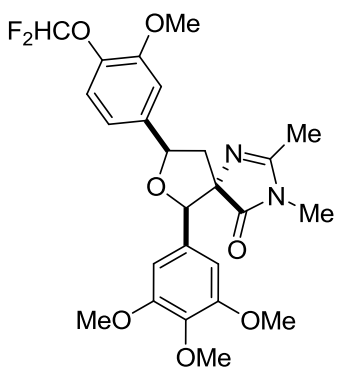

${ }^{1} \mathrm{H}$ NMR $\left(700 \mathrm{MHz}, 303 \mathrm{~K}, \mathrm{CDCl}_{3}\right): \delta=7.59(\mathrm{~d}, J=1.8 \mathrm{~Hz}, 1 \mathrm{H}), 7.16(\mathrm{~d}, J=8.1 \mathrm{~Hz}$, $1 \mathrm{H}), 7.13(\mathrm{dd}, J=8.1,1.8 \mathrm{~Hz}, 1 \mathrm{H}), 6.61(\mathrm{~s}, 2 \mathrm{H}), 6.56(\mathrm{t}, J=75.3 \mathrm{~Hz}, 1 \mathrm{H}), 5.25(\mathrm{t}, J=$ $8.0 \mathrm{~Hz}, 1 \mathrm{H}), 5.07(\mathrm{~s}, 1 \mathrm{H}), 3.97(\mathrm{~s}, 3 \mathrm{H}), 3.82(\mathrm{~s}, 6 \mathrm{H}), 3.81(\mathrm{~s}, 3 \mathrm{H}), 2.76(\mathrm{dd}, J=13.4$, $8.0 \mathrm{~Hz}, 1 \mathrm{H}), 2.71(\mathrm{~s}, 3 \mathrm{H}), 2.39(\mathrm{dd}, J=13.4,8.1 \mathrm{~Hz}, 1 \mathrm{H}), 2.18(\mathrm{~s}, 3 \mathrm{H})$.

${ }^{13} \mathrm{C}$ NMR $\left(175 \mathrm{MHz}, 303 \mathrm{~K}, \mathrm{CDCl}_{3}\right): \delta=182.2(\mathrm{C}=0), 162.3(\mathrm{C}), 152.9\left(2 \times \mathrm{O}-\mathrm{C}_{\mathrm{Ar}}\right)$, $151.5(\mathrm{C}), 140.0(\mathrm{C}), 139.8(\mathrm{t}, J=3.2 \mathrm{~Hz}, \mathrm{C}), 137.9(\mathrm{C}), 130.9(\mathrm{C}), 122.2\left(\mathrm{CH}_{\mathrm{Ar}}\right), 119.6$

$\left(\mathrm{CH}_{\mathrm{Ar}}\right), 116.4\left(\mathrm{t}, J=259.5 \mathrm{~Hz}, \mathrm{CHF}_{2}\right), 111.8\left(\mathrm{CH}_{\mathrm{Ar}}\right), 103.3\left(2 \times \mathrm{CH}_{\mathrm{Ar}}\right), 86.4(\mathrm{CH}-\mathrm{O}), 80.2$ (CH-O), $79.9(\mathrm{C}), 61.0\left(\mathrm{OCH}_{3}\right), 56.2\left(2 \times \mathrm{OCH}_{3}\right), 56.1\left(\mathrm{OCH}_{3}\right), 44.0\left(\mathrm{CH}_{2}\right), 26.4\left(\mathrm{CH}_{3}\right), 15.7\left(\mathrm{CH}_{3}\right)$.

HRMS (ESI) m/z: $\left[\mathrm{M}+\mathrm{H}^{+}\right]$Calcd for $\mathrm{C}_{25} \mathrm{H}_{29} \mathrm{~F}_{2} \mathrm{~N}_{2} \mathrm{O}_{7}$ 507.1937, found 507.1940.

$\left(5 R^{*}, 6 R^{*}, 8 R^{*}\right)-8$-(4-(Difluoromethoxy)-3-methoxyphenyl)-2,3-dimethyl-6-(3,4,5-trimethoxyphenyl)-7oxa-1,3-diazaspiro[4.4]non-1-en-4-one 3'ea

Contains $9 \mathrm{w} \%$ of EtOH. Yield $52 \mathrm{mg}$. Corrected yield $47 \mathrm{mg}(0.09 \mathrm{mmol}, 31 \%)$. Yellowish crystals, $\mathrm{mp}=$ $136-138^{\circ} \mathrm{C}(\mathrm{EtOH}) ; \mathrm{R}_{f}=0.15$ (EtOAc, UV/vanillin viz.).

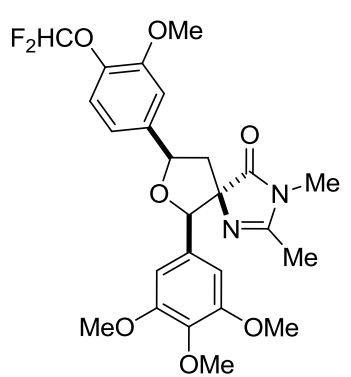

${ }^{1} \mathrm{H}$ NMR $\left(700 \mathrm{MHz}, 303 \mathrm{~K}, \mathrm{CDCl}_{3}\right): \delta=7.44(\mathrm{~d}, J=1.9 \mathrm{~Hz}, 1 \mathrm{H}), 7.15(\mathrm{~d}, J=8.1 \mathrm{~Hz}$, $1 \mathrm{H}), 7.12(\mathrm{dd}, J=8.1,1.9 \mathrm{~Hz}, 1 \mathrm{H}), 6.55(\mathrm{t}, J=75.3 \mathrm{~Hz}, 1 \mathrm{H}), 6.51(\mathrm{~s}, 2 \mathrm{H}), 5.28(\mathrm{dd}, J$ $=8.8,6.6 \mathrm{~Hz}, 1 \mathrm{H}), 5.07(\mathrm{~s}, 1 \mathrm{H}), 3.92(\mathrm{~s}, 3 \mathrm{H}), 3.81(\mathrm{~s}, 3 \mathrm{H}), 3.80(\mathrm{~s}, 6 \mathrm{H}), 2.99(\mathrm{~s}, 3 \mathrm{H})$, $2.93(\mathrm{dd}, J=13.2,8.8 \mathrm{~Hz}, 1 \mathrm{H}), 2.21(\mathrm{dd}, J=13.2,6.6 \mathrm{~Hz}, 1 \mathrm{H}), 1.95(\mathrm{~s}, 3 \mathrm{H})$.

${ }^{13} \mathrm{C}$ NMR (175 MHz, $\left.303 \mathrm{~K}, \mathrm{CDCl}_{3}\right): \delta=182.5(\mathrm{C}=\mathrm{O}), 159.6(\mathrm{C}), 152.9\left(2 \times \mathrm{O}-\mathrm{C}_{\mathrm{Ar}}\right)$, $151.3\left(\mathrm{O}-\mathrm{C}_{\mathrm{Ar}}\right), 140.7(\mathrm{C}), 139.7(\mathrm{t}, J=3.1 \mathrm{~Hz}, \mathrm{C}), 137.8(\mathrm{C}), 131.0(\mathrm{C}), 122.3\left(\mathrm{CH}_{\mathrm{Ar}}\right)$, $119.4\left(\mathrm{CH}_{\mathrm{Ar}}\right), 116.4\left(\mathrm{t}, J=259.6 \mathrm{~Hz}, \mathrm{CHF}_{2}\right), 111.8\left(\mathrm{CH}_{\mathrm{Ar}}\right), 103.9\left(2 \times \mathrm{CH}_{\mathrm{Ar}}\right), 89.0(\mathrm{CH}-$ O), $80.1(\mathrm{CH}-\mathrm{O}), 79.1(\mathrm{C}), 61.0\left(\mathrm{OCH}_{3}\right), 56.3\left(2 \times \mathrm{OCH}_{3}\right), 56.1\left(\mathrm{OCH}_{3}\right), 44.7\left(\mathrm{CH}_{2}\right), 26.8\left(\mathrm{CH}_{3}\right), 15.4\left(\mathrm{CH}_{3}\right)$.

HRMS (ESI) m/z: $\left[\mathrm{M}+\mathrm{H}^{+}\right]$Calcd for $\mathrm{C}_{25} \mathrm{H}_{29} \mathrm{~F}_{2} \mathrm{~N}_{2} \mathrm{O}_{7}$ 507.1937, found 507.1938. 
$\left(5 S^{*}, 6 R^{*}, 8 R^{*}\right)$-2,3-Dimethyl-8-phenyl-6-(3,4,5-trimethoxyphenyl)-7-oxa-1,3-diazaspiro[4.4]non-1-en-4one $3 \mathrm{fa}$

Yield $36 \mathrm{mg}(0.09 \mathrm{mmol}, 29 \%)$. Colorless crystals, $\mathrm{mp}=137-139{ }^{\circ} \mathrm{C}\left(\mathrm{Et}_{2} \mathrm{O}\right) ; \mathrm{R}_{f}=0.05(\mathrm{EtOAc}, \mathrm{UV} /$ vanillin viz.).

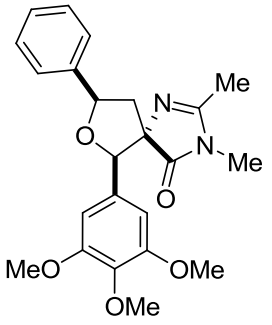

${ }^{1} \mathrm{H}$ NMR $\left(700 \mathrm{MHz}, 303 \mathrm{~K}, \mathrm{CDCl}_{3}\right): \delta=7.68(\mathrm{dd}, J=8.0,1.2 \mathrm{~Hz}, 2 \mathrm{H}), 7.42(\mathrm{t}, J=7.7 \mathrm{~Hz}$, $2 \mathrm{H}), 7.36(\mathrm{tt}, J=7.4,1.2 \mathrm{~Hz}, 1 \mathrm{H}), 6.61(\mathrm{~s}, 2 \mathrm{H}), 5.28(\mathrm{t}, J=8.1 \mathrm{~Hz}, 1 \mathrm{H}), 5.06(\mathrm{~s}, 1 \mathrm{H}), 3.83$ $(\mathrm{s}, 6 \mathrm{H}), 3.81(\mathrm{~s}, 3 \mathrm{H}), 2.72(\mathrm{~s}, 3 \mathrm{H}), 2.70(\mathrm{dd}, J=13.3,7.4 \mathrm{~Hz}, 1 \mathrm{H}), 2.44(\mathrm{dd}, J=13.3,8.9$ $\mathrm{Hz}, 1 \mathrm{H}), 2.19(\mathrm{~s}, 3 \mathrm{H})$.

${ }^{13} \mathrm{C}$ NMR $\left(175 \mathrm{MHz}, 303 \mathrm{~K}, \mathrm{CDCl}_{3}\right): \delta=182.1$ (C=O), 162.0 (C), $152.8\left(2 \times \mathrm{O}-\mathrm{C}_{\mathrm{Ar}}\right), 140.8$ (C), 137.9 (C), $131.4(\mathrm{C}), 128.7\left(2 \times \mathrm{CH}_{\mathrm{Ar}}\right), 128.2\left(\mathrm{CH}_{\mathrm{Ar}}\right), 127.2\left(2 \times \mathrm{CH}_{\mathrm{Ar}}\right), 103.4\left(2 \times \mathrm{CH}_{\mathrm{Ar}}\right)$, $86.6(\mathrm{CH}-\mathrm{O}), 80.9(\mathrm{CH}-\mathrm{O}), 80.0(\mathrm{C}), 61.0\left(\mathrm{OCH}_{3}\right), 56.3\left(2 \times \mathrm{OCH}_{3}\right), 44.5\left(\mathrm{CH}_{2}\right), 26.4\left(\mathrm{CH}_{3}\right), 15.7\left(\mathrm{CH}_{3}\right)$.

HRMS (ESI) m/z: [M+H'] Calcd for $\mathrm{C}_{23} \mathrm{H}_{27} \mathrm{~N}_{2} \mathrm{O}_{5} 411.1914$, found 411.1918.

$\left(5 R^{*}, 6 R^{*}, 8 R^{*}\right)-2,3-D i m e t h y l-8-p h e n y l-6-(3,4,5-t r i m e t h o x y p h e n y l)-7-o x a-1,3-d i a z a s p i r o[4.4]$ non-1-en4-one $3^{\prime}$ fa

Yield $37 \mathrm{mg}$ (0.09 mmol, 30\%). Colorless crystals, $\mathrm{mp}=145^{\circ} \mathrm{C}\left(\mathrm{Et}_{2} \mathrm{O}\right) ; \mathrm{R}_{f}=0.22$ (EtOAc, UV/vanillin viz.).

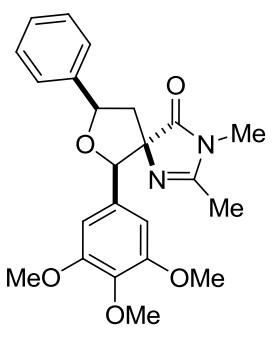

${ }^{1} \mathrm{H} \mathrm{NMR}\left(700 \mathrm{MHz}, 303 \mathrm{~K}, \mathrm{CDCl}_{3}\right): \delta=7.61(\mathrm{~d}, J=7.1 \mathrm{~Hz}, 2 \mathrm{H}), 7.39(\mathrm{t}, J=7.9 \mathrm{~Hz}, 2 \mathrm{H})$, $7.32(\mathrm{t}, J=7.3 \mathrm{~Hz}, 1 \mathrm{H}), 6.52(\mathrm{~s}, 2 \mathrm{H}), 5.30(\mathrm{t}, J=8.0 \mathrm{~Hz}, 1 \mathrm{H}), 5.07(\mathrm{~s}, 1 \mathrm{H}), 3.82(\mathrm{~s}, 6 \mathrm{H})$, $3.81(\mathrm{~s}, 3 \mathrm{H}), 3.00(\mathrm{~s}, 3 \mathrm{H}), 2.90(\mathrm{dd}, J=13.2,8.5 \mathrm{~Hz}, 1 \mathrm{H}), 2.26(\mathrm{dd}, J=13.2,7.5 \mathrm{~Hz}, 1 \mathrm{H})$, $1.96(\mathrm{~s}, 3 \mathrm{H})$.

${ }^{13} \mathrm{C} \mathrm{NMR}\left(175 \mathrm{MHz}, 303 \mathrm{~K}, \mathrm{CDCl}_{3}\right): \delta=183.0(\mathrm{C}=\mathrm{O}), 159.5$ (C), $152.9\left(2 \times \mathrm{O}-\mathrm{C}_{\mathrm{Ar}}\right), 141.4$ (C), $137.7(\mathrm{C}), 131.4(\mathrm{C}), 128.6\left(2 \times \mathrm{CH}_{\mathrm{Ar}}\right), 128.1\left(\mathrm{CH}_{\mathrm{Ar}}\right), 127.0\left(2 \times \mathrm{CH}_{\mathrm{Ar}}\right), 103.9\left(2 \times \mathrm{CH}_{\mathrm{Ar}}\right)$, $89.0(\mathrm{CH}-\mathrm{O}), 80.7(\mathrm{CH}-\mathrm{O}), 79.3(\mathrm{C}), 61.0\left(\mathrm{OCH}_{3}\right), 56.3\left(2 \times \mathrm{OCH}_{3}\right), 45.0\left(\mathrm{CH}_{2}\right), 26.8\left(\mathrm{CH}_{3}\right), 15.3\left(\mathrm{CH}_{3}\right)$.

HRMS (ESI) m/z: [M+H'] Calcd for $\mathrm{C}_{23} \mathrm{H}_{27} \mathrm{~N}_{2} \mathrm{O}_{5} 411.1914$, found 411.1913 . 


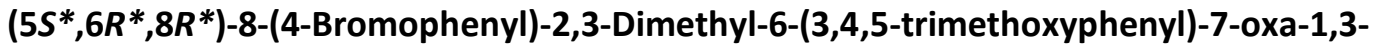
diazaspiro[4.4]non-1-en-4-one 3ga

Yield $43 \mathrm{mg}$. Contains $12 \%$ of impurity. Corrected yield $38 \mathrm{mg}(0.08 \mathrm{mmol}, 26 \%)$. Yellowish crystals; $\mathrm{R}_{f}=$ 0.03 (EtOAc, UV/vanillin viz.).

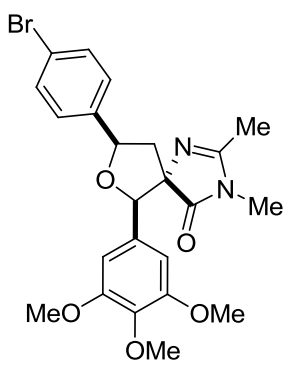

${ }^{1} \mathrm{H}$ NMR $\left(700 \mathrm{MHz}, 303 \mathrm{~K}, \mathrm{CDCl}_{3}\right): \delta=7.57(\mathrm{~d}, J=8.5 \mathrm{~Hz}, 2 \mathrm{H}), 7.54(\mathrm{~d}, J=8.5 \mathrm{~Hz}, 2 \mathrm{H})$, $6.59(\mathrm{~s}, 2 \mathrm{H}), 5.23(\mathrm{dd}, J=8.1 \mathrm{~Hz}, 1 \mathrm{H}), 5.05(\mathrm{~s}, 1 \mathrm{H}), 3.83(\mathrm{~s}, 6 \mathrm{H}), 3.81(\mathrm{~s}, 3 \mathrm{H}), 2.72(\mathrm{dd}$, $J=13.4,7.6 \mathrm{~Hz}, 1 \mathrm{H}), 2.71(\mathrm{~s}, 3 \mathrm{H}), 2.36(\mathrm{dd}, J=13.4,8.5 \mathrm{~Hz}, 1 \mathrm{H}), 2.18(\mathrm{~s}, 3 \mathrm{H})$.

${ }^{13} \mathrm{C} \mathrm{NMR}\left(175 \mathrm{MHz}, 303 \mathrm{~K}, \mathrm{CDCl}_{3}\right): \delta=182.1(\mathrm{C}=\mathrm{O}), 151.2(\mathrm{C}), 152.9\left(2 \times \mathrm{O}-\mathrm{C}_{\mathrm{Ar}}\right), 140.0$ (C), $138.0(\mathrm{C}), 131.8\left(2 \times \mathrm{CH}_{\mathrm{Ar}}\right), 131.0(\mathrm{C}), 128.9\left(2 \times \mathrm{CH}_{\mathrm{Ar}}\right), 122.1(\mathrm{C}), 103.3\left(2 \times \mathrm{CH}_{\mathrm{Ar}}\right), 86.5$ (CH-O), $79.8(\mathrm{CH}-\mathrm{O}), 79.8(\mathrm{C}), 61.0\left(\mathrm{OCH}_{3}\right), 56.3\left(2 \times \mathrm{OCH}_{3}\right), 44.2\left(\mathrm{CH}_{2}\right), 26.4\left(\mathrm{CH}_{3}\right), 15.7$ $\left(\mathrm{CH}_{3}\right)$.

HRMS (ESI) m/z: $\left[\mathrm{M}+\mathrm{H}^{+}\right]$Calcd for $\mathrm{C}_{23} \mathrm{H}_{26}{ }^{79} \mathrm{BrN}_{2} \mathrm{O}_{5} 489.1020$, for $\mathrm{C}_{23} \mathrm{H}_{26}{ }^{81} \mathrm{BrN}_{2} \mathrm{O}_{5}$ 491.1003, found 489.1021, 491.1005.

$\left(5 R^{*}, 6 R^{*}, 8 R^{*}\right)-8-(4-B r o m o p h e n y l)-2,3-D i m e t h y l-6-(3,4,5-t r i m e t h o x y p h e n y l)-7-o x a-1,3-$ diazaspiro[4.4]non-1-en-4-one 3'ga

Yield $40 \mathrm{mg}(0.08 \mathrm{mmol}, 27 \%)$. Colorless crystals; $\mathrm{mp}=173-175^{\circ} \mathrm{C}\left(\mathrm{Et}_{2} \mathrm{O}\right) ; \mathrm{R}_{f}=0.18(\mathrm{EtOAc}, \mathrm{UV} /$ vanillin viz.).

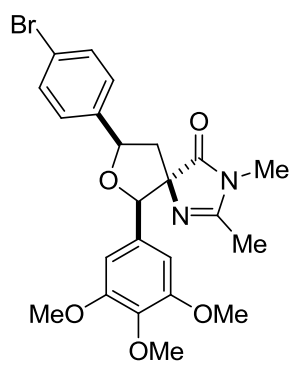

${ }^{1} \mathrm{H} \mathrm{NMR}\left(700 \mathrm{MHz}, 303 \mathrm{~K}, \mathrm{CDCl}_{3}\right): \delta=7.53-7.47(\mathrm{~m}, 4 \mathrm{H}), 6.50(\mathrm{~s}, 2 \mathrm{H}), 5.26(\mathrm{dd}, \mathrm{J}=8.6$, $7.0 \mathrm{~Hz}, 1 \mathrm{H}), 5.05(\mathrm{~s}, 1 \mathrm{H}), 3.81(\mathrm{~s}, 9 \mathrm{H}), 2.99(\mathrm{~s}, 3 \mathrm{H}), 2.91(\mathrm{dd}, \mathrm{J}=13.2,8.6 \mathrm{~Hz}, 1 \mathrm{H}), 2.19$ $(\mathrm{dd}, J=13.2,7.0 \mathrm{~Hz}, 1 \mathrm{H}), 1.96(\mathrm{~s}, 3 \mathrm{H})$.

${ }^{13} \mathrm{C} \mathrm{NMR}\left(175 \mathrm{MHz}, 303 \mathrm{~K}, \mathrm{CDCl}_{3}\right): \delta=182.6$ (C=O), 159.6 (C), $152.9\left(2 \times \mathrm{O}-\mathrm{C}_{\mathrm{Ar}}\right), 140.7$ (C), $137.8(\mathrm{C}), 131.7\left(2 \times \mathrm{CH}_{\mathrm{Ar}}\right), 131.0(\mathrm{C}), 128.7\left(2 \times \mathrm{CH}_{\mathrm{Ar}}\right), 121.9$ (C), $103.9\left(2 \times \mathrm{CH}_{\mathrm{Ar}}\right)$, $89.0(\mathrm{CH}-\mathrm{O}), 79.9(\mathrm{CH}-\mathrm{O}), 79.2(\mathrm{C}), 61.0\left(\mathrm{OCH}_{3}\right), 56.3\left(2 \times \mathrm{OCH}_{3}\right), 44.8\left(\mathrm{CH}_{2}\right), 26.8\left(\mathrm{CH}_{3}\right)$, $15.3\left(\mathrm{CH}_{3}\right)$.

HRMS (ESI) m/z: [M+H'] Calcd for $\mathrm{C}_{23} \mathrm{H}_{26}{ }^{79} \mathrm{BrN}_{2} \mathrm{O}_{5}$ 489.1020, for $\mathrm{C}_{23} \mathrm{H}_{26}{ }^{81} \mathrm{BrN}_{2} \mathrm{O}_{5}$ 491.1003, found 489.1020, 491.1003. 
$\left(5 S^{*}, 6 R^{*}, 8 R^{*}\right)-2,3-D i m e t h y l-8-(t h i o p h e n-2-y l)-6-(3,4,5-t r i m e t h o x y p h e n y l)-7-o x a-1,3-$ diazaspiro[4.4]non-1-en-4-one 3ha

Yield $48 \mathrm{mg}(0.11 \mathrm{mmol}, 38 \%)$. Colorless crystals; $\mathrm{mp}=161-162{ }^{\circ} \mathrm{C}\left(\mathrm{Et}_{2} \mathrm{O}\right) ; \mathrm{R}_{f}=0.05(\mathrm{EtOAc}, \mathrm{UV} /$ vanillin viz.).

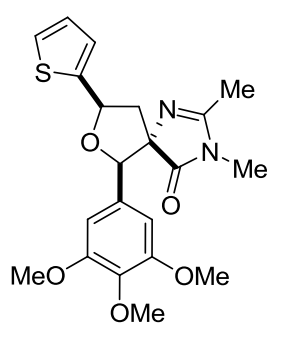

${ }^{1} \mathrm{H}$ NMR $\left(700 \mathrm{MHz}, 303 \mathrm{~K}, \mathrm{CDCl}_{3}\right): \delta=7.34(\mathrm{dd}, J=5.1,1.2 \mathrm{~Hz}, 1 \mathrm{H}), 7.21(\mathrm{dd}, J=3.5,1.2$ $\mathrm{Hz}, 1 \mathrm{H}), 7.02(\mathrm{dd}, J=5.1,3.5 \mathrm{~Hz}, 1 \mathrm{H}), 6.58(\mathrm{~s}, 2 \mathrm{H}), 5.58(\mathrm{dd}, J=8.8,7.2 \mathrm{~Hz}, 1 \mathrm{H}), 5.04(\mathrm{~s}$, $1 \mathrm{H}), 3.82(\mathrm{~s}, 6 \mathrm{H}), 3.81(\mathrm{~s}, 3 \mathrm{H}), 2.74(\mathrm{~s}, 3 \mathrm{H}), 2.70(\mathrm{dd}, J=13.2,7.2 \mathrm{~Hz}, 1 \mathrm{H}), 2.56$ (dd, $J=$ $13.2,8.8 \mathrm{~Hz}, 1 \mathrm{H}), 2.19(\mathrm{~s}, 3 \mathrm{H})$.

${ }^{13} \mathrm{C}$ NMR $\left(175 \mathrm{MHz}, 303 \mathrm{~K}, \mathrm{CDCl}_{3}\right): \delta=181.5$ (C=O), 162.1 (C), $152.8\left(2 \times \mathrm{O}-\mathrm{C}_{\mathrm{Ar}}\right), 143.7$

(C), 137.9 (C), $131.3(\mathrm{C}), 126.7\left(\mathrm{CH}_{\mathrm{Ar}}\right), 125.8\left(\mathrm{CH}_{\mathrm{Ar}}\right), 125.7\left(\mathrm{CH}_{\mathrm{Ar}}\right), 103.5\left(2 \times \mathrm{CH}_{\mathrm{Ar}}\right), 86.7$ (CH-O), $79.8(\mathrm{CH}-\mathrm{O}), 76.5(\mathrm{C}), 61.0\left(\mathrm{OCH}_{3}\right), 56.3\left(2 \times \mathrm{OCH}_{3}\right), 44.6\left(\mathrm{CH}_{2}\right), 26.4\left(\mathrm{CH}_{3}\right), 15.8\left(\mathrm{CH}_{3}\right)$.

HRMS (ESI) m/z: [M+H'] Calcd for $\mathrm{C}_{21} \mathrm{H}_{25} \mathrm{~N}_{2} \mathrm{O}_{5} \mathrm{~S}$ 417.1479, found 417.1479.

$\left(5 R^{*}, 6 R^{*}, 8 R^{*}\right)$-2,3-Dimethyl-8-(thiophen-2-yl)-6-(3,4,5-trimethoxyphenyl)-7-oxa-1,3diazaspiro[4.4]non-1-en-4-one 3'ha

Yield $40 \mathrm{mg}(0.10 \mathrm{mmol}, 32 \%)$. Brown crystals; $\mathrm{mp}=105-106{ }^{\circ} \mathrm{C}\left(\mathrm{CHCl}_{3} /\right.$ hexane); $\mathrm{R}_{f}=0.18$ (EtOAc, UV/vanillin viz.).

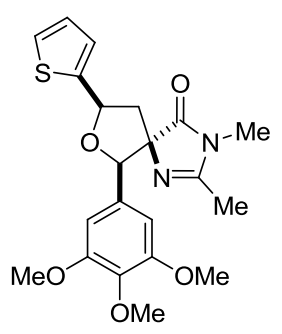

${ }^{1} \mathrm{H}$ NMR $\left(700 \mathrm{MHz}, 303 \mathrm{~K}, \mathrm{CDCl}_{3}\right): \delta=7.31(\mathrm{dd}, J=5.1,1.2 \mathrm{~Hz}, 1 \mathrm{H}), 7.16(\mathrm{dd}, J=3.4,1.2$ $\mathrm{Hz}, 1 \mathrm{H}), 7.00(\mathrm{dd}, J=5.1,3.4 \mathrm{~Hz}, 1 \mathrm{H}), 6.50(\mathrm{~s}, 2 \mathrm{H}), 5.0(\mathrm{dd}, J=8.3,7.1 \mathrm{~Hz}, 1 \mathrm{H}), 5.06(\mathrm{~s}$, $1 \mathrm{H}), 3.81(\mathrm{~s}, 6 \mathrm{H}), 3.80(\mathrm{~s}, 3 \mathrm{H}), 3.00(\mathrm{~s}, 3 \mathrm{H}), 2.91(\mathrm{dd}, J=13.2,8.3 \mathrm{~Hz}, 1 \mathrm{H}), 2.40(\mathrm{dd}, J=$ $13.2,7.1 \mathrm{~Hz}, 1 \mathrm{H}), 1.96(\mathrm{~s}, 3 \mathrm{H})$.

${ }^{13} \mathrm{C} \mathrm{NMR}\left(75 \mathrm{MHz}, 303 \mathrm{~K}, \mathrm{CDCl}_{3}\right): \delta=181.5$ (C=O), 160.1 (C), $152.8\left(2 \times 0-\mathrm{C}_{\mathrm{Ar}}\right), 144.4(\mathrm{C})$, $137.6(\mathrm{C}), 131.0(\mathrm{C}), 126.8\left(\mathrm{CH}_{\mathrm{Ar}}\right), 125.7\left(\mathrm{CH}_{\mathrm{Ar}}\right), 125.4\left(\mathrm{CH}_{\mathrm{Ar}}\right), 103.7\left(2 \times \mathrm{CH}_{\mathrm{Ar}}\right), 88.8(\mathrm{CH}-$ O), $78.9(\mathrm{CH}-\mathrm{O}), 76.4(\mathrm{C}), 60.9\left(\mathrm{OCH}_{3}\right), 56.2\left(2 \times \mathrm{OCH}_{3}\right), 44.8\left(\mathrm{CH}_{2}\right), 26.8\left(\mathrm{CH}_{3}\right), 15.2\left(\mathrm{CH}_{3}\right)$.

HRMS (ESI) m/z: [M+H'] Calcd for $\mathrm{C}_{21} \mathrm{H}_{25} \mathrm{~N}_{2} \mathrm{O}_{5} \mathrm{~S}$ 417.1479, found 417.1485. 
$\left(5 S^{*}, 6 R^{*}, 8 R^{*}\right)$-2,3-Dimethyl-8-(furan-2-yl)-6-(3,4,5-trimethoxyphenyl)-7-oxa-1,3-diazaspiro[4.4]non-1en-4-one 3ia

Yield $33 \mathrm{mg}$ (0.08 mmol, 28\%). Amorphous solid; $\mathrm{R}_{f}=0.04$ (EtOAc, UV/vanillin viz.).

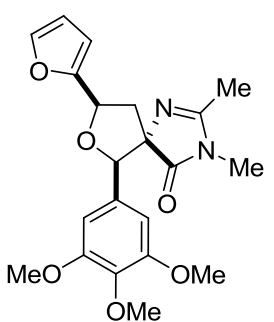

${ }^{1} \mathrm{H}$ NMR $\left(700 \mathrm{MHz}, 303 \mathrm{~K}, \mathrm{CDCl}_{3}\right): \delta=7.48(\mathrm{~d}, J=1.8,0.9 \mathrm{~Hz}, 1 \mathrm{H}), 6.57(\mathrm{dd}, J=3.3,0.9$ $\mathrm{Hz}, 1 \mathrm{H}), 6.54(\mathrm{~s}, 2 \mathrm{H}), 6.41(\mathrm{dd}, J=3.3,1.8 \mathrm{~Hz}, 1 \mathrm{H}), 5.34(\mathrm{dd}, J=9.4,7.0 \mathrm{~Hz}, 1 \mathrm{H}), 5.00(\mathrm{~s}$, $1 \mathrm{H}), 3.81(\mathrm{~s}, 6 \mathrm{H}), 3.80(\mathrm{~s}, 3 \mathrm{H}), 2.76(\mathrm{~s}, 3 \mathrm{H}), 2.74(\mathrm{dd}, J=13.1,9.4 \mathrm{~Hz}, 1 \mathrm{H}), 2.53(\mathrm{dd}, J=$ $13.1,7.0 \mathrm{~Hz}, 1 \mathrm{H}), 2.20(\mathrm{~s}, 3 \mathrm{H})$.

${ }^{13} \mathrm{C}$ NMR (175 MHz, $\left.303 \mathrm{~K}, \mathrm{CDCl}_{3}\right): \delta=181.4(\mathrm{C}=\mathrm{O}), 162.1$ (C), $152.8\left(2 \times \mathrm{O}-\mathrm{C}_{\mathrm{Ar}}\right), 152.5$ (C), $142.9\left(\mathrm{CH}_{\mathrm{Ar}}\right), 137.9(\mathrm{C}), 131.4(\mathrm{C}), 110.6\left(\mathrm{CH}_{\mathrm{Ar}}\right), 108.9\left(\mathrm{CH}_{\mathrm{Ar}}\right), 103.5\left(2 \times \mathrm{CH}_{\mathrm{Ar}}\right), 86.7$ (CH-O), $79.5(\mathrm{C}), 73.9(\mathrm{CH}-\mathrm{O}), 61.0\left(\mathrm{OCH}_{3}\right), 56.3\left(2 \times \mathrm{OCH}_{3}\right), 40.5\left(\mathrm{CH}_{2}\right), 26.4\left(\mathrm{CH}_{3}\right), 15.7\left(\mathrm{CH}_{3}\right)$.

HRMS (ESI) m/z: [M+H'] Calcd for $\mathrm{C}_{21} \mathrm{H}_{25} \mathrm{~N}_{2} \mathrm{O}_{6} 401.1707$, found 401.1707.

$\left(5 R^{*}, 6 R^{*}, 8 R^{*}\right)$-2,3-Dimethyl-8-(furan-2-yl)-6-(3,4,5-trimethoxyphenyl)-7-oxa-1,3-diazaspiro[4.4]non-1en-4-one 3'ia

Yield $30 \mathrm{mg}$ (0.08 mmol, 25\%). Brown crystals; $\mathrm{R}_{f}=0.11$ (EtOAc, UV/vanillin viz.).

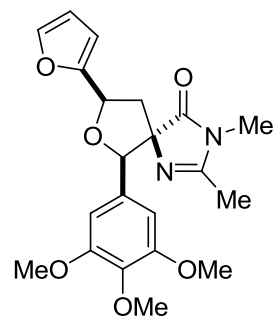

${ }^{1} \mathrm{H} \mathrm{NMR}\left(700 \mathrm{MHz}, 303 \mathrm{~K}, \mathrm{CDCl}_{3}\right): \delta=7.46(\mathrm{~d}, J=1.8,0.9 \mathrm{~Hz}, 1 \mathrm{H}), 6.51(\mathrm{dd}, J=3.2,0.9$ $\mathrm{Hz}, 1 \mathrm{H}), 6.45(\mathrm{~s}, 2 \mathrm{H}), 6.39(\mathrm{dd}, J=3.2,1.8 \mathrm{~Hz}, 1 \mathrm{H}), 5.34(\mathrm{t}, J=8.0 \mathrm{~Hz}, 1 \mathrm{H}), 5.01(\mathrm{~s}, 1 \mathrm{H})$, $3.79(\mathrm{~s}, 9 \mathrm{H}), 3.01(\mathrm{~s}, 3 \mathrm{H}), 2.76(\mathrm{dd}, J=13.2,8.4 \mathrm{~Hz}, 1 \mathrm{H}), 2.58(\mathrm{dd}, J=13.2,7.7 \mathrm{~Hz}, 1 \mathrm{H})$, $1.98(\mathrm{~s}, 3 \mathrm{H})$.

${ }^{13} \mathrm{C} \mathrm{NMR}\left(75 \mathrm{MHz}, 303 \mathrm{~K}, \mathrm{CDCl}_{3}\right): \delta=182.7(\mathrm{C}=\mathrm{O}), 160.0(\mathrm{C}), 152.8\left(2 \times \mathrm{O}-\mathrm{C}_{\mathrm{Ar}}\right), 152.5(\mathrm{C})$, $143.1(\mathrm{CH}), 137.5(\mathrm{C}), 131.0(\mathrm{C}), 110.5\left(\mathrm{CH}_{\mathrm{Ar}}\right), 109.0\left(\mathrm{CH}_{\mathrm{Ar}}\right), 103.5\left(2 \times \mathrm{CH}_{\mathrm{Ar}}\right), 88.7(\mathrm{CH}-$ O), $78.8(\mathrm{C}), 73.6(\mathrm{CH}-\mathrm{O}), 60.9\left(\mathrm{OCH}_{3}\right), 56.2\left(2 \times \mathrm{OCH}_{3}\right), 40.7\left(\mathrm{CH}_{2}\right), 26.8\left(\mathrm{CH}_{3}\right), 15.3\left(\mathrm{CH}_{3}\right)$.

HRMS (ESI) m/z: [M+H'] Calcd for $\mathrm{C}_{21} \mathrm{H}_{25} \mathrm{~N}_{2} \mathrm{O}_{6}$ 401.1707, found 401.1711. 
$\left(5 S^{*}, 6 R^{*}, 8 R^{*}\right)-8-($ Anthracen-9-yl)-2,3-dimethyl-6-(3,4,5-trimethoxyphenyl)-7-oxa-1,3diazaspiro[4.4]non-1-en-4-one 3ja

Yield $72 \mathrm{mg}(0.14 \mathrm{mmol}, 47 \%), d r$ n.d. Unstable in solution and at r.t. Colorless crystals; $\mathrm{R}_{f}=0.10$ (hexane/EtOAc = 1/1, UV/vanillin viz.).

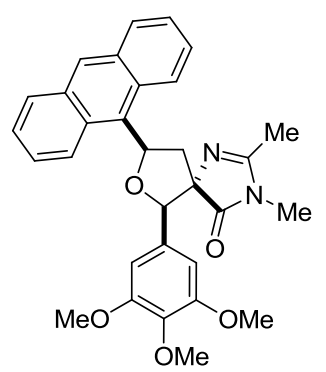

${ }^{1} \mathrm{H}$ NMR $\left(700 \mathrm{MHz}, 303 \mathrm{~K}, \mathrm{CDCl}_{3}\right): \delta=9.01(\mathrm{~d}, J=9.0 \mathrm{~Hz}, 2 \mathrm{H}), 8.48(\mathrm{~s}, 1 \mathrm{H}), 8.04(\mathrm{dd}$, $J=8.4,1.4 \mathrm{~Hz}, 2 \mathrm{H}), 7.57(\mathrm{ddd}, J=9.0,6.4,1.4 \mathrm{~Hz}, 2 \mathrm{H}), 7.48(\mathrm{dd}, J=8.4,6.4 \mathrm{~Hz}, 1 \mathrm{H})$, $6.71(\mathrm{t}, J=9.1 \mathrm{~Hz}, 1 \mathrm{H}), 6.64(\mathrm{~s}, 2 \mathrm{H}), 5.24(\mathrm{~s}, 1 \mathrm{H}), 3.83^{9}(\mathrm{~s}, 6 \mathrm{H}), 3.83^{6}(\mathrm{~s}, 3 \mathrm{H}), 3.07(\mathrm{~s}$, $3 \mathrm{H}), 3.02(\mathrm{dd}, J=13.5,8.6 \mathrm{~Hz}, 1 \mathrm{H}), 2.85$ (dd, $J=13.5,9.5 \mathrm{~Hz}, 1 \mathrm{H}), 2.01$ (br s, 3H).

${ }^{13} \mathrm{C} \mathrm{NMR}\left(175 \mathrm{MHz}, 303 \mathrm{~K}, \mathrm{CDCl}_{3}\right): \delta=183.6$ (C=O), $159.1(\mathrm{C}), 152.9\left(2 \times \mathrm{O}-\mathrm{C}_{\mathrm{Ar}}\right), 137.8$ $\left(\mathrm{C}_{\mathrm{Ar}}\right), 131.8\left(2 \times \mathrm{C}_{\mathrm{Ar}}\right), 131.4\left(\mathrm{C}_{\mathrm{Ar}}\right), 130.0\left(2 \times \mathrm{C}_{\mathrm{Ar}}\right), 129.8\left(\mathrm{C}_{\mathrm{Ar}}\right), 129.4\left(2 \times \mathrm{CH}_{\mathrm{Ar}}\right), 129.1$ $\left(\mathrm{CH}_{\mathrm{Ar}}\right), 125.8\left(2 \times \mathrm{CH}_{\mathrm{Ar}}\right), 125.5\left(2 \times \mathrm{CH}_{\mathrm{Ar}}\right), 125.0\left(2 \times \mathrm{CH}_{\mathrm{Ar}}\right), 103.7\left(2 \times \mathrm{CH}_{\mathrm{Ar}}\right), 89.6(\mathrm{CH}-\mathrm{O})$, $79.8(\mathrm{C}), 77.7(\mathrm{CH}-\mathrm{O}), 61.0\left(\mathrm{OCH}_{3}\right), 56.3\left(2 \times \mathrm{OCH}_{3}\right), 43.7\left(\mathrm{CH}_{2}\right), 26.9\left(\mathrm{CH}_{3}\right), 15.4\left(\mathrm{CH}_{3}\right)$.

HRMS (ESI) m/z: [M+H'] Calcd for $\mathrm{C}_{31} \mathrm{H}_{31} \mathrm{~N}_{2} \mathrm{O}_{5}$ 511.2227, found 511.2230. 
(5S*,6R*,8R*)-2-Ethyl-8-(4-methoxyphenyl)-3-methyl-6-(3,4,5-trimethoxyphenyl)-7-oxa-1,3diazaspiro[4.4]non-1-en-4-one 3ka

Yield $58 \mathrm{mg}$ (0.13 mmol, 43\%). Colorless crystals; $\mathrm{R}_{f}=0.12$ (EtOAc, UV/vanillin viz.).

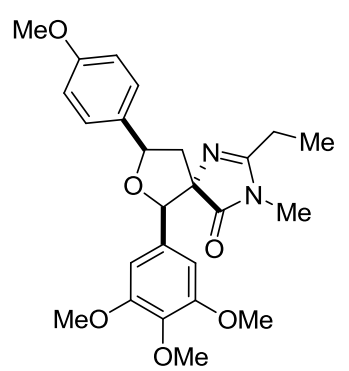

${ }^{1} \mathrm{H}$ NMR $\left(700 \mathrm{MHz}, 303 \mathrm{~K}, \mathrm{CDCl}_{3}\right): \delta=7.64(\mathrm{~d}, J=8.6 \mathrm{~Hz}, 2 \mathrm{H}), 6.95(\mathrm{~d}, J=8.6 \mathrm{~Hz}$, $2 \mathrm{H}), 6.59(\mathrm{~s}, 2 \mathrm{H}), 5.21(\mathrm{t}, J=8.1 \mathrm{~Hz}, 1 \mathrm{H}), 5.05(\mathrm{~s}, 1 \mathrm{H}), 3.82^{4}(\mathrm{~s}, 3 \mathrm{H}), 3.82^{0}(\mathrm{~s}, 6 \mathrm{H})$, $3.80(\mathrm{~s}, 3 \mathrm{H}), 2.71(\mathrm{dd}, J=13.4,7.5 \mathrm{~Hz}, 1 \mathrm{H}), 2.70(\mathrm{~s}, 3 \mathrm{H}), 2.49-2.32(\mathrm{~m}, 3 \mathrm{H}), 1.32(\mathrm{t}$, $J=7.5 \mathrm{~Hz}, 3 \mathrm{H})$.

${ }^{13} \mathrm{C} \mathrm{NMR}\left(175 \mathrm{MHz}, 303 \mathrm{~K}, \mathrm{CDCl}_{3}\right): \delta=182.5(\mathrm{C}=\mathrm{O}), 165.9$ (C), 159.6 (C), 152.7 (2×O$\left.\mathrm{C}_{\mathrm{Ar}}\right), 137.8(\mathrm{C}), 133.0(\mathrm{C}), 131.3(\mathrm{C}), 128.7\left(2 \times \mathrm{CH}_{\mathrm{Ar}}\right), 114.0\left(2 \times \mathrm{CH}_{\mathrm{Ar}}\right), 103.3\left(2 \times \mathrm{CH}_{\mathrm{Ar}}\right)$, $86.4(\mathrm{CH}-\mathrm{O}), 80.6(\mathrm{CH}-\mathrm{O}), 79.8(\mathrm{C}), 60.9\left(\mathrm{OCH}_{3}\right), 56.2\left(2 \times \mathrm{OCH}_{3}\right), 55.4\left(\mathrm{OCH}_{3}\right), 44.2$ $\left(\mathrm{CH}_{2}\right), 26.1\left(\mathrm{CH}_{3}\right), 22.3\left(\mathrm{CH}_{2}\right), 9.8\left(\mathrm{CH}_{3}\right)$.

HRMS (ESI) m/z: [M+H'] Calcd for $\mathrm{C}_{25} \mathrm{H}_{31} \mathrm{~N}_{2} \mathrm{O}_{6} 455.2177$, found 455.2177.

$\left(5 R^{*}, 6 R^{*}, 8 R^{*}\right)$-2-Ethyl-8-(4-methoxyphenyl)-3-methyl-6-(3,4,5-trimethoxyphenyl)-7-oxa-1,3diazaspiro[4.4]non-1-en-4-one 3'ka

Yield $45 \mathrm{mg}(0.10 \mathrm{mmol}, 33 \%)$. Colorless crystals; $\mathrm{mp}=98-99{ }^{\circ} \mathrm{C}$ (hexane/EtOAc); $\mathrm{R}_{f}=0.37$ (EtOAc, UV/vanillin viz.).

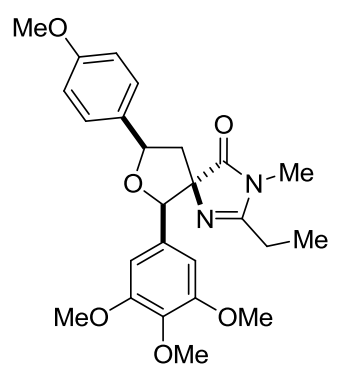

${ }^{1} \mathrm{H}$ NMR $\left(700 \mathrm{MHz}, 303 \mathrm{~K}, \mathrm{CDCl}_{3}\right): \delta=7.59(\mathrm{~d}, J=8.6 \mathrm{~Hz}, 2 \mathrm{H}), 6.93(\mathrm{~d}, J=8.6 \mathrm{~Hz}$, $2 \mathrm{H}), 6.50(\mathrm{~s}, 2 \mathrm{H}), 5.26(\mathrm{dd}, J=8.5,7.1 \mathrm{~Hz}, 1 \mathrm{H}), 5.04(\mathrm{~s}, 1 \mathrm{H}), 3.82(\mathrm{~s}, 3 \mathrm{H}), 3.81(\mathrm{~s}$, $6 \mathrm{H}), 3.79(\mathrm{~s}, 3 \mathrm{H}), 2.98(\mathrm{~s}, 3 \mathrm{H}), 2.88(\mathrm{dd}, J=13.2,8.5 \mathrm{~Hz}, 1 \mathrm{H}), 2.29(\mathrm{dq}, J=15.2,7.5$ $\mathrm{Hz}, 1 \mathrm{H}), 2.24(\mathrm{dd}, J=13.2,7.1 \mathrm{~Hz}, 1 \mathrm{H}), 2.17(\mathrm{dq}, J=15.2,7.5 \mathrm{~Hz}, 1 \mathrm{H}), 0.92(\mathrm{t}, J=7.5$ $\mathrm{Hz}, 3 \mathrm{H})$.

${ }^{13} \mathrm{C} \mathrm{NMR}\left(75 \mathrm{MHz}, 303 \mathrm{~K}, \mathrm{CDCl}_{3}\right): \delta=183.0(\mathrm{C}=\mathrm{O}), 163.6$ (C), 159.5 (C), 152.7 (2×O$\left.\mathrm{C}_{\mathrm{Ar}}\right), 137.5(\mathrm{C}), 133.5(\mathrm{C}), 131.3(\mathrm{C}), 128.6\left(2 \times \mathrm{CH}_{\mathrm{Ar}}\right), 113.9\left(2 \times \mathrm{CH}_{\mathrm{Ar}}\right), 103.7\left(2 \times \mathrm{CH}_{\mathrm{Ar}}\right)$, $89.2(\mathrm{CH}-\mathrm{O}), 80.7(\mathrm{CH}-\mathrm{O}), 79.0(\mathrm{C}), 60.9\left(\mathrm{OCH}_{3}\right), 56.2\left(2 \times \mathrm{OCH}_{3}\right), 55.4\left(\mathrm{OCH}_{3}\right), 44.6\left(\mathrm{CH}_{2}\right), 26.6\left(\mathrm{CH}_{3}\right), 22.0$ $\left(\mathrm{CH}_{2}\right), 9.9\left(\mathrm{CH}_{3}\right)$.

HRMS (ESI) m/z: [M+H'] Calcd for $\mathrm{C}_{25} \mathrm{H}_{31} \mathrm{~N}_{2} \mathrm{O}_{6}$ 455.2177, found 455.2177. 
$\left(5 S^{*}, 6 R^{*}, 8 R^{*}\right)$-3-Cyclohexyl-8-(4-methoxyphenyl)-2-methyl-6-(3,4,5-trimethoxyphenyl)-7-oxa-1,3diazaspiro[4.4]non-1-en-4-one 3la

Yield $70 \mathrm{mg}$ (0.14 mmol, 46\%). Viscous oil; $\mathrm{R}_{f}=0.08$ (EtOAc, UV/vanillin viz.).

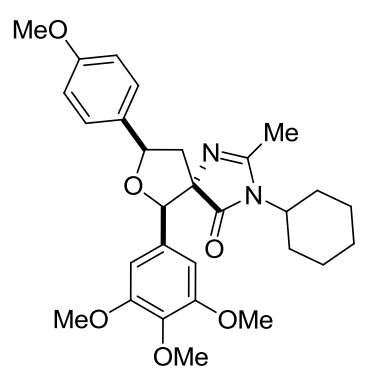

${ }^{1} \mathrm{H}$ NMR $\left(700 \mathrm{MHz}, 303 \mathrm{~K}, \mathrm{CDCl}_{3}\right): \delta=7.64(\mathrm{~d}, J=8.6 \mathrm{~Hz}, 2 \mathrm{H}), 6.95(\mathrm{~d}, J=8.6 \mathrm{~Hz}$, $2 \mathrm{H}), 6.61(\mathrm{~s}, 2 \mathrm{H}), 5.21(\mathrm{t}, J=8.2 \mathrm{~Hz}, 1 \mathrm{H}), 4.98(\mathrm{~s}, 1 \mathrm{H}), 3.83(\mathrm{~s}, 6 \mathrm{H}), 3.82(\mathrm{~s}, 3 \mathrm{H})$, $3.78(\mathrm{~s}, 3 \mathrm{H}), 3.20(\mathrm{tt}, J=12.3,3.9 \mathrm{~Hz}, 1 \mathrm{H}), 2.70(\mathrm{dd}, J=13.4,7.8 \mathrm{~Hz}, 1 \mathrm{H}), 2.42(\mathrm{dd}$, $J=13.4,8.5 \mathrm{~Hz}, 1 \mathrm{H}), 2.20(\mathrm{~s}, 3 \mathrm{H}), 1.84(\mathrm{dq}, J=12.5,3.8 \mathrm{~Hz}, 1 \mathrm{H}), 1.75-1.70(\mathrm{~m}$, $1 \mathrm{H}), 1.68-1.64(\mathrm{~m}, 1 \mathrm{H}), 1.61-1.54(\mathrm{~m}, 2 \mathrm{H}), 1.33-1.23(\mathrm{~m}, 2 \mathrm{H}), 1.18-1.02(\mathrm{~m}, 3 \mathrm{H})$, $0.96-0.91(\mathrm{~m}, 1 \mathrm{H})$.

${ }^{13} \mathrm{C}$ NMR (175 MHz, $\left.303 \mathrm{~K}, \mathrm{CDCl}_{3}\right): \delta=182.7$ (C=0), 162.5 (C), $159.6(\mathrm{C}), 152.8$ $\left(2 \times 0-\mathrm{C}_{\mathrm{Ar}}\right), 138.0(\mathrm{C}), 133.1(\mathrm{C}), 131.0(\mathrm{C}), 128.7\left(2 \times \mathrm{CH}_{\mathrm{Ar}}\right), 114.0\left(2 \times \mathrm{CH}_{\mathrm{Ar}}\right), 103.4\left(2 \times \mathrm{CH}_{\mathrm{Ar}}\right), 86.5(\mathrm{CH}-\mathrm{O}), 80.7$ (CH-O), $79.2(\mathrm{C}), 60.9\left(\mathrm{OCH}_{3}\right), 56.3\left(2 \times \mathrm{OCH}_{3}\right), 55.4\left(\mathrm{OCH}_{3}\right), 53.8(\mathrm{CHN}), 43.6\left(\mathrm{CH}_{2}\right), 29.9\left(\mathrm{CH}_{2}\right), 29.1\left(\mathrm{CH}_{2}\right)$, $25.9^{6}\left(\mathrm{CH}_{2}\right), 25.9^{3}\left(\mathrm{CH}_{2}\right), 25.9\left(\mathrm{CH}_{2}\right), 16.9\left(\mathrm{CH}_{3}\right)$.

HRMS (ESI) m/z: [M+H'] Calcd for $\mathrm{C}_{29} \mathrm{H}_{37} \mathrm{~N}_{2} \mathrm{O}_{6}$ 509.2646, found 509.2646.

$\left(5 R^{*}, 6 R^{*}, 8 R^{*}\right)-3-C y c l o h e x y l-8-(4-m e t h o x y p h e n y l)-2-m e t h y l-6-(3,4,5-t r i m e t h o x y p h e n y l)-7-o x a-1,3-$ diazaspiro[4.4]non-1-en-4-one 3 'la

Yield $58 \mathrm{mg}(0.11 \mathrm{mmol}, 37 \%)$. Colorless crystals, $\mathrm{mp}=124-125{ }^{\circ} \mathrm{C}$ (hexane/EtOAc); $\mathrm{R}_{f}=0.33$ (EtOAc, UV/vanillin viz.).

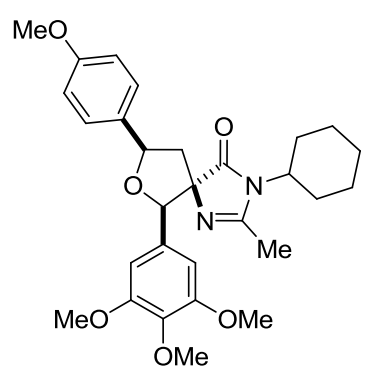

${ }^{1} \mathrm{H} \mathrm{NMR}\left(700 \mathrm{MHz}, 303 \mathrm{~K}, \mathrm{CDCl}_{3}\right): \delta=7.56(\mathrm{~d}, J=8.7 \mathrm{~Hz}, 2 \mathrm{H}), 6.92(\mathrm{~d}, J=8.7 \mathrm{~Hz}$, $2 \mathrm{H}), 6.53(\mathrm{~s}, 2 \mathrm{H}), 5.22(\mathrm{t}, J=7.9 \mathrm{~Hz}, 1 \mathrm{H}), 5.00(\mathrm{~s}, 1 \mathrm{H}), 3.82(\mathrm{~s}, 9 \mathrm{H}), 3.80(\mathrm{~s}, 3 \mathrm{H})$, $3.46(\mathrm{tt}, J=12.4,3.9 \mathrm{~Hz}, 1 \mathrm{H}), 2.83(\mathrm{dd}, J=13.2,8.5 \mathrm{~Hz}, 1 \mathrm{H}), 2.22(\mathrm{dd}, J=13.2$, $7.3 \mathrm{~Hz}, 1 \mathrm{H}), 2.08-1.99(\mathrm{~m}, 2 \mathrm{H}), 1.98(\mathrm{~s}, 3 \mathrm{H}), 1.96-1.80(\mathrm{~m}, 2 \mathrm{H}), 1.71-1.40(\mathrm{~m}$, $3 \mathrm{H}), 1.36-1.09(\mathrm{~m}, 3 \mathrm{H})$.

${ }^{13} \mathrm{C} \mathrm{NMR}\left(75 \mathrm{MHz}, 303 \mathrm{~K}, \mathrm{CDCl}_{3}\right): \delta=183.1(\mathrm{C}=0), 159.9$ (C), 159.5 (C), 152.7 $\left(2 \times \mathrm{O}-\mathrm{C}_{\mathrm{Ar}}\right), 137.6(\mathrm{C}), 133.5(\mathrm{C}), 131.2(\mathrm{C}), 128.5\left(2 \times \mathrm{CH}_{\mathrm{Ar}}\right), 114.0\left(2 \times \mathrm{CH}_{\mathrm{Ar}}\right), 104.1$ $\left(2 \times \mathrm{CH}_{\mathrm{Ar}}\right), 89.3(\mathrm{CH}-\mathrm{O}), 80.5(\mathrm{CH}-\mathrm{O}), 78.6(\mathrm{C}), 61.0\left(\mathrm{OCH}_{3}\right), 56.2\left(2 \times \mathrm{OCH}_{3}\right), 55.4\left(\mathrm{OCH}_{3}\right), 54.2(\mathrm{CHN}), 44.8$ $\left(\mathrm{CH}_{2}\right), 30.3\left(\mathrm{CH}_{2}\right), 30.2\left(\mathrm{CH}_{2}\right), 25.1\left(2 \times \mathrm{CH}_{2}\right), 25.1\left(\mathrm{CH}_{2}\right), 16.7\left(\mathrm{CH}_{3}\right)$.

HRMS (ESI) m/z: [M+H'] Calcd for $\mathrm{C}_{29} \mathrm{H}_{37} \mathrm{~N}_{2} \mathrm{O}_{6}$ 509.2646, found 509.2652. 
$\left(5 S^{*}, 6 R^{*}, 8 R^{*}\right)$-3-Benzyl-8-(4-methoxyphenyl)-2-methyl-6-(3,4,5-trimethoxyphenyl)-7-oxa-1,3diazaspiro[4.4]non-1-en-4-one $3 \mathrm{ma}$

Yield $70 \mathrm{mg}(0.14 \mathrm{mmol}, 45 \%)$. Yellowish solid; $\mathrm{mp}=128-130{ }^{\circ} \mathrm{C}\left(\mathrm{Et}_{2} \mathrm{O} / \mathrm{hexane}\right) ; \mathrm{R}_{f}=0.12$ (EtOAc, UV/vanillin viz.).

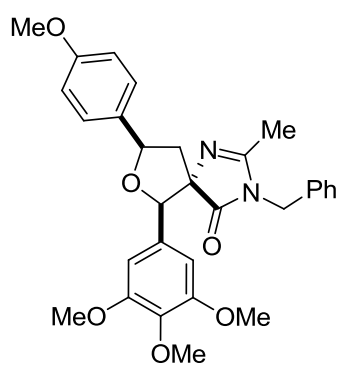

${ }^{1} \mathrm{H}$ NMR $\left(700 \mathrm{MHz}, 303 \mathrm{~K}, \mathrm{CDCl}_{3}\right): \delta=7.64(\mathrm{~d}, J=8.6 \mathrm{~Hz}, 2 \mathrm{H}), 7.22-7.16(\mathrm{~m}, 3 \mathrm{H})$, $6.96(\mathrm{~d}, J=8.6 \mathrm{~Hz}, 2 \mathrm{H}), 6.66(\mathrm{~s}, 2 \mathrm{H}), 6.87(\mathrm{dd}, J=7.8,2.2 \mathrm{~Hz}, 2 \mathrm{H}), 5.25(\mathrm{t}, J=8.1$ $\mathrm{Hz}, 1 \mathrm{H}), 5.11(\mathrm{~s}, 1 \mathrm{H}), 4.73(\mathrm{~d}, J=15.9 \mathrm{~Hz}, 1 \mathrm{H}), 4.09(\mathrm{~d}, J=16.1 \mathrm{~Hz}, 1 \mathrm{H}), 3.86(\mathrm{~s}, 3 \mathrm{H})$, $3.83(\mathrm{~s}, 3 \mathrm{H}), 3.74(\mathrm{~s}, 6 \mathrm{H}), 2.74(\mathrm{dd}, J=13.2,7.5 \mathrm{~Hz}, 1 \mathrm{H}), 2.49$ (dd, $J=13.2,8.7 \mathrm{~Hz}$, $1 \mathrm{H}), 2.01(\mathrm{~s}, 3 \mathrm{H})$.

${ }^{13} \mathrm{C} \mathrm{NMR}\left(175 \mathrm{MHz}, 303 \mathrm{~K}, \mathrm{CDCl}_{3}\right): \delta=182.3(\mathrm{C}=\mathrm{O}), 162.1$ (C), 159.7 (C), $153.0(2 \times \mathrm{O}-$ $\left.\mathrm{C}_{\mathrm{Ar}}\right), 138.1(\mathrm{C}), 135.5(\mathrm{C}), 133.9(\mathrm{C}), 131.1(\mathrm{C}), 129.0\left(2 \times \mathrm{CH}_{\mathrm{Ar}}\right), 128.6\left(2 \times \mathrm{CH}_{\mathrm{Ar}}\right), 128.0$ $\left(\mathrm{CH}_{\mathrm{Ar}}\right), 126.6\left(2 \times \mathrm{CH}_{\mathrm{Ar}}\right), 114.1\left(2 \times \mathrm{CH}_{\mathrm{Ar}}\right), 103.8\left(2 \times \mathrm{CH}_{\mathrm{Ar}}\right), 86.5(\mathrm{CH}-\mathrm{O}), 80.5(\mathrm{CH}-\mathrm{O}), 79.7(\mathrm{C}), 61.0\left(\mathrm{OCH}_{3}\right), 56.2$ $\left(2 \times \mathrm{OCH}_{3}\right), 55.4\left(\mathrm{OCH}_{3}\right), 45.1\left(\mathrm{CH}_{2}\right), 43.7\left(\mathrm{CH}_{2}\right), 16.1\left(\mathrm{CH}_{3}\right)$.

HRMS (ESI) m/z: [M+H'] Calcd for $\mathrm{C}_{30} \mathrm{H}_{33} \mathrm{~N}_{2} \mathrm{O}_{6}$ 517.2333, found 517.2331.

$\left(5 R^{*}, 6 R^{*}, 8 R^{*}\right)$-3-Benzyl-8-(4-methoxyphenyl)-2-methyl-6-(3,4,5-trimethoxyphenyl)-7-oxa-1,3diazaspiro[4.4]non-1-en-4-one 3 'ma

Yield $48 \mathrm{mg}(0.09 \mathrm{mmol}, 31 \%)$. Colorless crystals, $\mathrm{mp}=154-155^{\circ} \mathrm{C}\left(\mathrm{Et}_{2} \mathrm{O}\right) ; \mathrm{R}_{f}=0.47(\mathrm{EtOAc}, \mathrm{UV} /$ vanillin viz.).

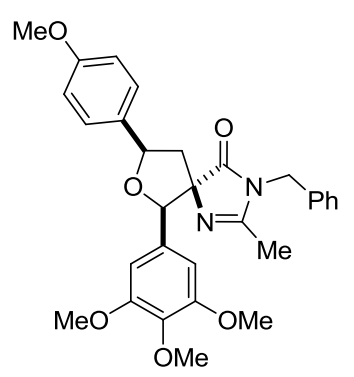

${ }^{1} \mathrm{H}$ NMR $\left(700 \mathrm{MHz}, 303 \mathrm{~K}, \mathrm{CDCl}_{3}\right): \delta=7.58(\mathrm{~d}, J=8.4 \mathrm{~Hz}, 2 \mathrm{H}), 7.27-7.20(\mathrm{~m}, 3 \mathrm{H})$, $6.93(\mathrm{~d}, J=8.4 \mathrm{~Hz}, 2 \mathrm{H}), 6.87(\mathrm{~d}, J=6.0 \mathrm{~Hz}, 2 \mathrm{H}), 6.60(\mathrm{~s}, 2 \mathrm{H}), 5.28(\mathrm{t}, J=7.8 \mathrm{~Hz}, 1 \mathrm{H})$, $5.09(\mathrm{~s}, 1 \mathrm{H}), 4.77(\mathrm{~d}, J=16.1 \mathrm{~Hz}, 1 \mathrm{H}), 4.47(\mathrm{~d}, J=16.1 \mathrm{~Hz}, 1 \mathrm{H}), 3.82(\mathrm{~s}, 6 \mathrm{H}), 3.76(\mathrm{~s}$, $6 \mathrm{H}), 2.94(\mathrm{dd}, J=13.2,8.6 \mathrm{~Hz}, 1 \mathrm{H}), 2.29(\mathrm{dd}, J=13.2,7.0 \mathrm{~Hz}, 1 \mathrm{H}), 1.87(\mathrm{~s}, 3 \mathrm{H})$.

${ }^{13} \mathrm{C} \mathrm{NMR}\left(175 \mathrm{MHz}, 303 \mathrm{~K}, \mathrm{CDCl}_{3}\right): \delta=182.7(\mathrm{C}=\mathrm{O}), 159.6(\mathrm{C}), 159.4(\mathrm{C}), 152.8(2 \times \mathrm{O}-$ $\left.\mathrm{C}_{\mathrm{Ar}}\right), 138.0(\mathrm{C}), 135.8(\mathrm{C}), 133.6(\mathrm{C}), 130.8(\mathrm{C}), 129.1\left(2 \times \mathrm{CH}_{\mathrm{Ar}}\right), 128.5\left(2 \times \mathrm{CH}_{\mathrm{Ar}}\right), 128.0$ $\left(\mathrm{CH}_{\mathrm{Ar}}\right), 126.5\left(2 \times \mathrm{CH}_{\mathrm{Ar}}\right), 114.0\left(2 \times \mathrm{CH}_{\mathrm{Ar}}\right), 104.9\left(2 \times \mathrm{CH}_{\mathrm{Ar}}\right), 89.1(\mathrm{CH}-\mathrm{O}), 80.4(\mathrm{CH}-\mathrm{O})$, $79.2(\mathrm{C}), 61.0\left(\mathrm{OCH}_{3}\right), 56.2\left(2 \times \mathrm{OCH}_{3}\right), 55.4\left(\mathrm{OCH}_{3}\right), 44.3\left(\mathrm{CH}_{2}\right), 43.9\left(\mathrm{CH}_{2}\right), 15.8\left(\mathrm{CH}_{3}\right)$.

HRMS (ESI) m/z: [M+H'] Calcd for $\mathrm{C}_{30} \mathrm{H}_{33} \mathrm{~N}_{2} \mathrm{O}_{6}$ 517.2333, found 517.2333. 


\section{Gram-scale experiment \& Further examples of stereoselective cycloaddition}

Procedure for gram-scale experiment: Anhydrous $\mathrm{TsOH}(0.95 \mathrm{~g}, 5.5 \mathrm{mmol}, 1.1$ equiv) was added in a single portion to a stirred solution of cyclopropane $1 \mathrm{a}(0.30 \mathrm{mmol}, 1$ equiv) and benzaldehyde $\mathbf{2} \mathbf{d}(1.59 \mathrm{~g}$, $15.0 \mathrm{mmol}, 3.0$ equiv) in anhydrous $\mathrm{CH}_{2} \mathrm{Cl}_{2}(15.0 \mathrm{~mL})$ at $0{ }^{\circ} \mathrm{C}$. A color of the solution gradually changed from colorless to a deep yellow. Reaction mixture was stirred for $3 \mathrm{~h}$ (TLC control) at $0{ }^{\circ} \mathrm{C}$. TMG $(0.94 \mathrm{~mL}$, $0.86 \mathrm{~g}, 7.5 \mathrm{mmol}, 1.5$ equiv) was added during $2 \mathrm{~min}$ and the solution was stirred for additional $1 \mathrm{~min}$. The resulting mixture was poured into separatory funnel with EtOAc $(60 \mathrm{~mL})$ and water $(30 \mathrm{~mL})$. The organic layer was separated and the aqueous layer was back-extracted with EtOAc $(20 \mathrm{~mL})$. Combined organic layers were washed with brine $(30 \mathrm{~mL})$, dried over anhydrous $\mathrm{Na} 2 \mathrm{SO} 4$ and concentrated in vacuo. The residue was subjected to column chromatography on silica (eluent hexane/EtOAc, 1/1 $\rightarrow$ EtOAc $\rightarrow$ EtOAc/iPrOH, 9/1) to furnish target 1,3-diazaspiro[4.4]non-1-en-4-ones 3'ad (less polar, $0.55 \mathrm{~g}, 1.6 \mathrm{mmol}$, $31 \%$ ) and $3 a d$ (more polar, $1.05 \mathrm{~g}, 3.0 \mathrm{mmol}, 60 \%$ ).

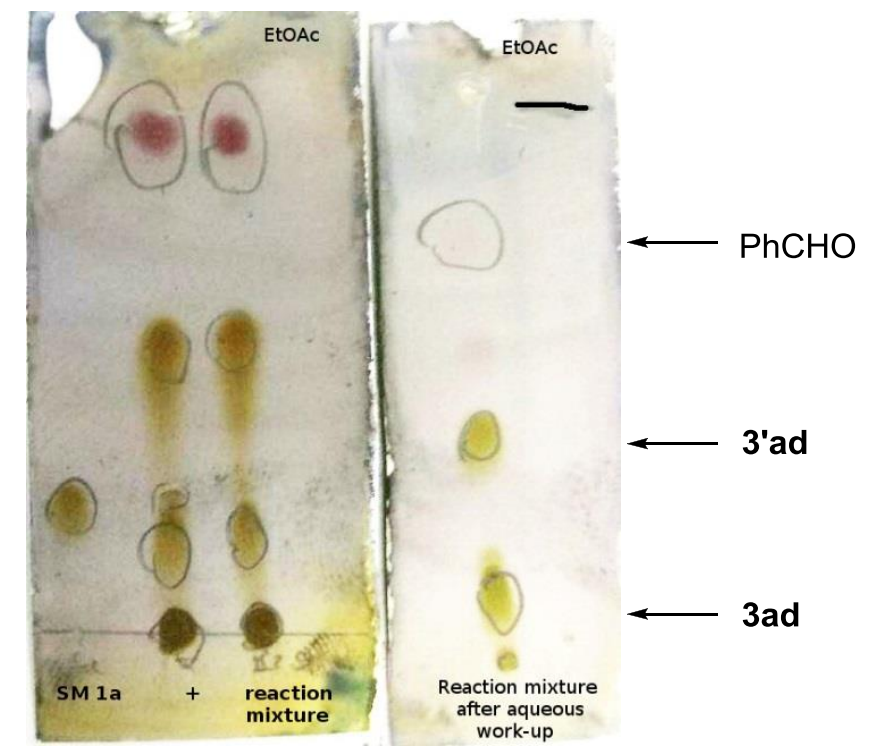

Figure SI1. TLC for gram-scale reaction.

NMR and other characterization data of products meet those, described for the reaction on $0.3 \mathrm{mmol}$ scale. 


\section{$1\left(5 S^{*}, 6 R^{*}, 8 R^{*}\right)$-6-(3-methoxy-4-nitrophenyl)-2,3-dimethyl-8-(3,4,5-trimethoxyphenyl)-7-oxa-1,3- diazaspiro[4.4]non-1-en-4-one 3cl}

Anhydrous TsOH ( $38 \mathrm{mg}, 0.22 \mathrm{mmol}, 1.1$ equiv) was added to a stirred solution of cyclopropane 1c (61 $\mathrm{mg}, 0.20 \mathrm{mmol}, 1$ equiv) and 4-nitro-3-methoxybenzaldehyde $2 \mathrm{l}$ (181 $\mathrm{mg}, 1.00 \mathrm{mmol}, 5.0$ equiv) in anhydrous $\mathrm{CH}_{2} \mathrm{Cl}_{2}\left(2.0 \mathrm{~mL}\right.$ ) at $0{ }^{\circ} \mathrm{C}$. Reaction mixture was stirred for $6 \mathrm{~h}$ at $0{ }^{\circ} \mathrm{C}$, quenched with TMG (38 $\mu \mathrm{L}, 34 \mathrm{mg}, 0.30 \mathrm{mmol}, 1.5$ equiv) and stirred for additional $10 \mathrm{~min}$. The resulting mixture was filtered through the short-pad of silica (elution with EtOAc/iPrOH, 9/1), concentrated on rotary evaporator and analyzed by ${ }^{1} \mathrm{H}$ NMR for $d r$, which was estimated to 3.8:1. The residue, combined with NMR-sample were subjected to column chromatography on silica (eluent EtOAc $\rightarrow \mathrm{EtOAc/iPrOH}, 9 / 1$ ) to give major isomer $3 \mathrm{cl}\left(49 \mathrm{mg}, 0.10 \mathrm{mmol}, 51 \%\right.$ ) as yellowish crystals with $\mathrm{mp}=180-182{ }^{\circ} \mathrm{C}$ (hexane/EtOAc).

$\mathrm{R}_{f}=0.07$ (EtOAc/iPrOH $=$ 9/1, UV/vanillin viz.).

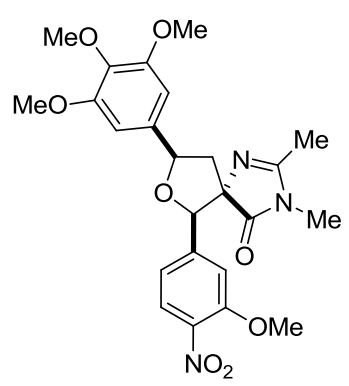

${ }^{1} \mathrm{H}$ NMR $\left(700 \mathrm{MHz}, 303 \mathrm{~K}, \mathrm{CDCl}_{3}\right): \delta=7.48(\mathrm{~d}, J=8.3 \mathrm{~Hz}, 1 \mathrm{H}), 7.23(\mathrm{~d}, J=1.5 \mathrm{~Hz}$, $1 \mathrm{H}), 6.98(\mathrm{~s}, 2 \mathrm{H}), 6.87(\mathrm{dd}, J=8.3,1.5 \mathrm{~Hz}, 1 \mathrm{H}), 5.25(\mathrm{t}, J=8.0 \mathrm{~Hz}, 1 \mathrm{H}), 5.17(\mathrm{~s}, 1 \mathrm{H})$, $3.94(\mathrm{~s}, 3 \mathrm{H}), 3.93(\mathrm{~s}, 6 \mathrm{H}), 3.87(\mathrm{~s}, 3 \mathrm{H}), 2.75(\mathrm{~s}, 3 \mathrm{H}), 2.75(\mathrm{dd}, J=13.4,7.6 \mathrm{~Hz}, 1 \mathrm{H})$, $2.46(\mathrm{dd}, J=13.4,8.5 \mathrm{~Hz}, 1 \mathrm{H}), 2.13(\mathrm{~s}, 3 \mathrm{H})$.

${ }^{13} \mathrm{C}$ NMR (175 MHz, $\left.303 \mathrm{~K}, \mathrm{CDCl}_{3}\right): \delta=181.8(\mathrm{C}=0), 162.8(\mathrm{C}), 153.6\left(2 \times \mathrm{O}-\mathrm{C}_{\mathrm{Ar}}\right)$, 153.0 (C), 143.2 (C), 139.1 (C), 139.2 (C), 136.0 (C), $125.5\left(\mathrm{CH}_{\mathrm{Ar}}\right), 117.3\left(\mathrm{CH}_{\mathrm{Ar}}\right)$, $111.6\left(\mathrm{CH}_{\mathrm{Ar}}\right), 104.4\left(2 \times \mathrm{CH}_{\mathrm{Ar}}\right), 85.1(\mathrm{CH}-\mathrm{O}), 81.4(\mathrm{CH}-\mathrm{O}), 79.8(\mathrm{C}), 61.0\left(\mathrm{OCH}_{3}\right), 56.6$ $\left(\mathrm{OCH}_{3}\right), 56.3\left(2 \times \mathrm{OCH}_{3}\right), 44.6\left(\mathrm{CH}_{2}\right), 26.5\left(\mathrm{CH}_{3}\right), 15.8\left(\mathrm{CH}_{3}\right)$.

HRMS (ESI) m/z: [M+H'] Calcd for $\mathrm{C}_{24} \mathrm{H}_{28} \mathrm{~N}_{3} \mathrm{O}_{8} 486.1871$, found 486.1873 .

\section{$\left(5 S^{*}, 6 R^{*}, 8 R^{*}\right)-2-$ Ethyl-8-(4-methoxyphenyl)-3-methyl-6-(2-phenyleth-1-yl)-7-oxa-1,3- diazaspiro[4.4]non-1-en-4-one $3 \mathrm{~km}$}

Anhydrous TsOH (47 mg, $0.28 \mathrm{mmol}, 1.1$ equiv) was added to a stirred solution of cyclopropane $\mathbf{1 k}$ (65 $\mathrm{mg}, 0.25 \mathrm{mmol}, 1$ equiv) and hydrocinnamic aldehyde $2 \mathrm{~m}$ (168 mg, $1.25 \mathrm{mmol}, 5.0$ equiv) in anhydrous $\mathrm{CH}_{2} \mathrm{Cl}_{2}(2.5 \mathrm{~mL})$ at r.t.. The reaction mixture was stirred for $24 \mathrm{~h}$ at r.t., quenched with TMG $(48 \mu \mathrm{L}, 43 \mathrm{mg}$, $0.38 \mathrm{mmol}, 1.5$ equiv) and stirred for additional $10 \mathrm{~min}$. The resulting mixture was filtered through the short-pad of silica (elution with EtOAc), concentrated on rotary evaporator and analyzed by ${ }^{1} \mathrm{H} N M R$ for $d r$, which was estimated to 4.8:1 (a number of by-productsm, resulting from self-condensation of $\mathbf{2 m}$, formed as well). The residue, combined with NMR-sample were subjected to column chromatography on silica (eluent hexane/EtOAc, 1/1 $\rightarrow$ EtOAc) to give major isomer $3 \mathbf{k m ~ ( 6 5 ~ m g , ~} 0.17 \mathrm{mmol}, 66 \%$ ) as colorless oil.

Yield $65 \mathrm{mg}$ ( $0.17 \mathrm{mmol}, 66 \%)$. Colorless oil; $\mathrm{R}_{f}=0.35$ (EtOAc, UV/vanillin viz.).

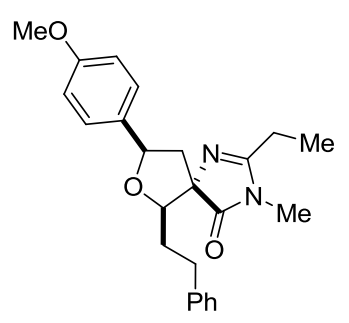

${ }^{1} \mathrm{H}$ NMR $\left(700 \mathrm{MHz}, 303 \mathrm{~K}, \mathrm{CDCl}_{3}\right): \delta=7.47(\mathrm{~d}, J=8.6 \mathrm{~Hz}, 2 \mathrm{H}), 7.25(\mathrm{t}, J=7.5 \mathrm{~Hz}$, $2 \mathrm{H}), 7.17-7.13(\mathrm{~m}, 3 \mathrm{H}), 6.90(\mathrm{~d}, J=8.6 \mathrm{~Hz}, 2 \mathrm{H}), 5.12(\mathrm{dd}, J=9.6,6.3 \mathrm{~Hz}, 1 \mathrm{H}), 4.11$ (dd, $J=9.4,4.0 \mathrm{~Hz}, 1 \mathrm{H}), 3.80(\mathrm{~s}, 3 \mathrm{H}), 3.02(\mathrm{~s}, 3 \mathrm{H}), 2.84$ (ddd, $J=13.8,10.5,5.4 \mathrm{~Hz}$, $1 \mathrm{H}$ ), 2.53 (ddd, $J=13.8,10.3,6.1 \mathrm{~Hz}, 1 \mathrm{H}$ ), 2.48 (q, $J=7.4 \mathrm{~Hz}, 2 \mathrm{H}$ ), 2.43 (dd, $J=13.0$, $6.3 \mathrm{~Hz}, 1 \mathrm{H}$ ), 2.28 (dd, $J=13.0,9.6 \mathrm{~Hz}, 1 \mathrm{H}$ ), 2.11 (ddd, $J=13.6,10.3,9.4,5.4 \mathrm{~Hz}$, $1 \mathrm{H}$ ), 1.56 (dddd, $J=13.6,10.5,6.1,4.0 \mathrm{~Hz}, 1 \mathrm{H}), 1.29(\mathrm{t}, J=7.4 \mathrm{~Hz}, 3 \mathrm{H})$. 
${ }^{13} \mathrm{C} \mathrm{NMR}\left(175 \mathrm{MHz}, 303 \mathrm{~K}, \mathrm{CDCl}_{3}\right): \delta=183.1$ (C=O), 164.9 (C), 159.5 (C), 141.9 (C), $132.9(\mathrm{C}), 128.6\left(2 \times \mathrm{CH}_{\mathrm{Ar}}\right)$, $128.4\left(2 \times \mathrm{CH}_{\mathrm{Ar}}\right), 128.3\left(2 \times \mathrm{CH}_{\mathrm{Ar}}\right), 125.9\left(\mathrm{CH}_{\mathrm{Ar}}\right), 114.0\left(2 \times \mathrm{CH}_{\mathrm{Ar}}\right), 84.2(\mathrm{CH}-\mathrm{O}), 80.4(\mathrm{CH}-\mathrm{O}), 79.4(\mathrm{C}), 55.4$ $\left(\mathrm{OCH}_{3}\right), 46.2\left(\mathrm{CH}_{2}\right), 33.0\left(\mathrm{CH}_{2}\right), 32.1\left(\mathrm{CH}_{2}\right), 26.5\left(\mathrm{CH}_{3}\right), 22.5\left(\mathrm{CH}_{2}\right), 9.7\left(\mathrm{CH}_{3}\right)$.

HRMS (ESI) m/z: [M+H+ Calcd for $\mathrm{C}_{24} \mathrm{H}_{29} \mathrm{~N}_{2} \mathrm{O}_{3}$ 393.2173, found 393.2173. 


\section{Experiment for competitive reactions}

Synthesis of cycloadduct: Anhydrous $\mathrm{Sn}(\mathrm{OTf})_{2}\left(2.5 \mathrm{mg}, 6^{*} 10^{-3} \mathrm{mmol}, 2 \% \mathrm{~mol}\right)$ was added to a stirred solution of cyclopropane SI-2 ( $79 \mathrm{mg}, 0.30 \mathrm{mmol}, 1$ equiv) and aldehyde $2 \mathrm{2a}$ ( $176 \mathrm{mg}, 0.90 \mathrm{mmol}, 3.0$ equiv) in anhydrous $\mathrm{CH}_{2} \mathrm{Cl}_{2}(3.0 \mathrm{~mL}$ ). Reaction mixture was stirred for $2 \mathrm{~h}$ (TLC control) and subjected to column chromatography on silica (eluent hexane/EtOAc $=4 / 1 \rightarrow 1 / 1$ ) to furnish target cycloadduct SI-3 (136 mg, $0.30 \mathrm{mmol}, 99 \%$ ) as colorless solid.

\section{$\left(2 R^{*}, 5 R^{*}\right)$-Dimethyl} dicarboxylate SI-3

5-(4-methoxyphenyl)-2-(3,4,5-trimethoxyphenyl)dihydrofuran-3,3(2H)-

Yield $136 \mathrm{mg}$ (0.30 mmol, 99\%). Colorless crystals; $\mathrm{mp}=124-125{ }^{\circ} \mathrm{C}$ (hexane/EtOAc); $\mathrm{R}_{f}=0.18$ (hexane/EtOAc $=4 / 1, \mathrm{UV} /$ vanillin viz.).<smiles></smiles>

${ }^{1} \mathrm{H} \mathrm{NMR}\left(700 \mathrm{MHz}, 303 \mathrm{~K}, \mathrm{CDCl}_{3}\right): \delta=7.48(\mathrm{~d}, J=8.7 \mathrm{~Hz}, 2 \mathrm{H}), 6.94(\mathrm{~d}, J=8.7$ $\mathrm{Hz}, 2 \mathrm{H}), 6.71(\mathrm{~s}, 2 \mathrm{H}), 5,68(\mathrm{~s}, 1 \mathrm{H}), 4.90(\mathrm{dd}, J=10.6,6.1 \mathrm{~Hz}, 1 \mathrm{H}), 3.84(\mathrm{~s}, 6 \mathrm{H})$, $3.83(\mathrm{~s}, 3 \mathrm{H}), 3.82(\mathrm{~s}, 3 \mathrm{H}), 3.81(\mathrm{~s}, 3 \mathrm{H}), 3.23(\mathrm{~s}, 3 \mathrm{H}), 3.00(\mathrm{dd}, J=13.0,10.6 \mathrm{~Hz}$, $1 \mathrm{H}), 2.68(\mathrm{dd}, J=13.6,6.1 \mathrm{~Hz}, 1 \mathrm{H})$.

${ }^{13} \mathrm{C}$ NMR $\left(175 \mathrm{MHz}, 303 \mathrm{~K}, \mathrm{CDCl}_{3}\right): \delta=171.5(\mathrm{C}=0), 169.5(\mathrm{C}=0), 159.7(\mathrm{C})$, $152.9\left(2 \times \mathrm{O}-\mathrm{C}_{\mathrm{Ar}}\right), 138.0$ (C), 133.4 (C), 132.0 (C), $128.1\left(2 \times \mathrm{CH}_{\mathrm{Ar}}\right), 114.1$ $\left(2 \times \mathrm{CH}_{\mathrm{Ar}}\right), 104.6\left(2 \times \mathrm{CH}_{\mathrm{Ar}}\right), 84.6(\mathrm{CH}-\mathrm{O}), 79.7(\mathrm{CH}-\mathrm{O}), 66.4(\mathrm{C}), 61.0\left(\mathrm{OCH}_{3}\right), 56.3\left(2 \times \mathrm{OCH}_{3}\right), 55.5\left(\mathrm{OCH}_{3}\right), 55.1$ $\left(\mathrm{OCH}_{3}\right), 52.6\left(\mathrm{OCH}_{3}\right), 42.5\left(\mathrm{CH}_{2}\right)$.

HRMS (ESI) m/z: $\left[\mathrm{M}+\mathrm{H}^{+}\right]$Calcd for $\mathrm{C}_{24} \mathrm{H}_{28} \mathrm{NaO}_{9} 483.1626$, found 483.1632.

Competitive reactions: Catalyst [anhydrous $\mathrm{Sn}(\mathrm{OTf})_{2}(6 \mathrm{mg}, 0.02 \mathrm{mmol}, 5 \% \mathrm{~mol})$ or anhydrous pTsOH (57 $\mathrm{mg}, 0.33 \mathrm{mmol}, 1.1$ equiv)] was added to a stirred solution of cyclopropane $1 \mathrm{a}$ ( $79 \mathrm{mg}, 0.30 \mathrm{mmol}, 1$ equiv), cyclopropane $\mathbf{S I - 2}$ (73 mg, $0.3 \mathrm{mmol}, 1$ equiv) and aldehyde $\mathbf{x}(176 \mathrm{mg}, 0.90 \mathrm{mmol}, 3.0$ equiv) in anhydrous $\mathrm{CH}_{2} \mathrm{Cl}_{2}\left(3.0 \mathrm{~mL}\right.$ ). Reaction mixture was stirred for $24 \mathrm{~h}$ (for $\mathrm{SnOTf}_{2}$ ) or $1 \mathrm{~h}$ (for $\mathrm{pTsOH}$, then quench with TMG) and filtered through short pad of silica (excessively eluted with EtOAc). Concentrated residue was analyzed by ${ }^{1} \mathrm{H} N M R$. 


\section{X-Ray analysis}

X-Ray quality crystals for the compound 3aa were prepared by interlayer diffusion of hexane into $\mathrm{CDCl}_{3}$ solution; for the compound 3'aa were prepared by slow evaporation of solvent from EtOAc solution.

Single crystal X-ray studies of 3aa were carried out in Center for molecule composition studies of INEOS RAS. X-ray datasets for $\mathbf{3}$ 'aa were collected in Kurchatov Centre for Synchrotron Radiation and Nanotechnology using 'Belok' beamline.

The structures were solved by direct method and refined in anisotropic approximation for non-hydrogen atoms. Hydrogens atoms of methyl, methylene and aromatic fragments were calculated according to those idealized geometry and refined with constraints applied to $\mathrm{C}-\mathrm{H}$ bond lengths and their equivalent displacement parameters $\left(\mathrm{U}_{\text {eq }}(\mathrm{H})=1.2 \mathrm{U}_{\text {eq }}(\mathrm{X}), \mathrm{C}\right.$ - central atom of $\mathrm{CH}_{2}$ group; $\mathrm{U}_{\text {eq }}(\mathrm{H})=1.5 \mathrm{U}_{\text {eq }}(\mathrm{C}), \mathrm{C}$ - central atom of $\mathrm{CH}_{3}$ group. All structures were solved with the ShelXT ${ }^{52}$ program and refined with the ShelXL ${ }^{53}$ program. Molecular graphics was drawn using OLEX2 ${ }^{54}$ program.

CCDC 1986173 and 1986174 contains the supplementary crystallographic data for 3aa and 3'aa. These data can be obtained free of charge from The Cambridge Crystallographic Data Centre via https://www.ccdc.cam.ac.uk/structures. 
Table S12. Crystallographic data for 3aa and 3'aa

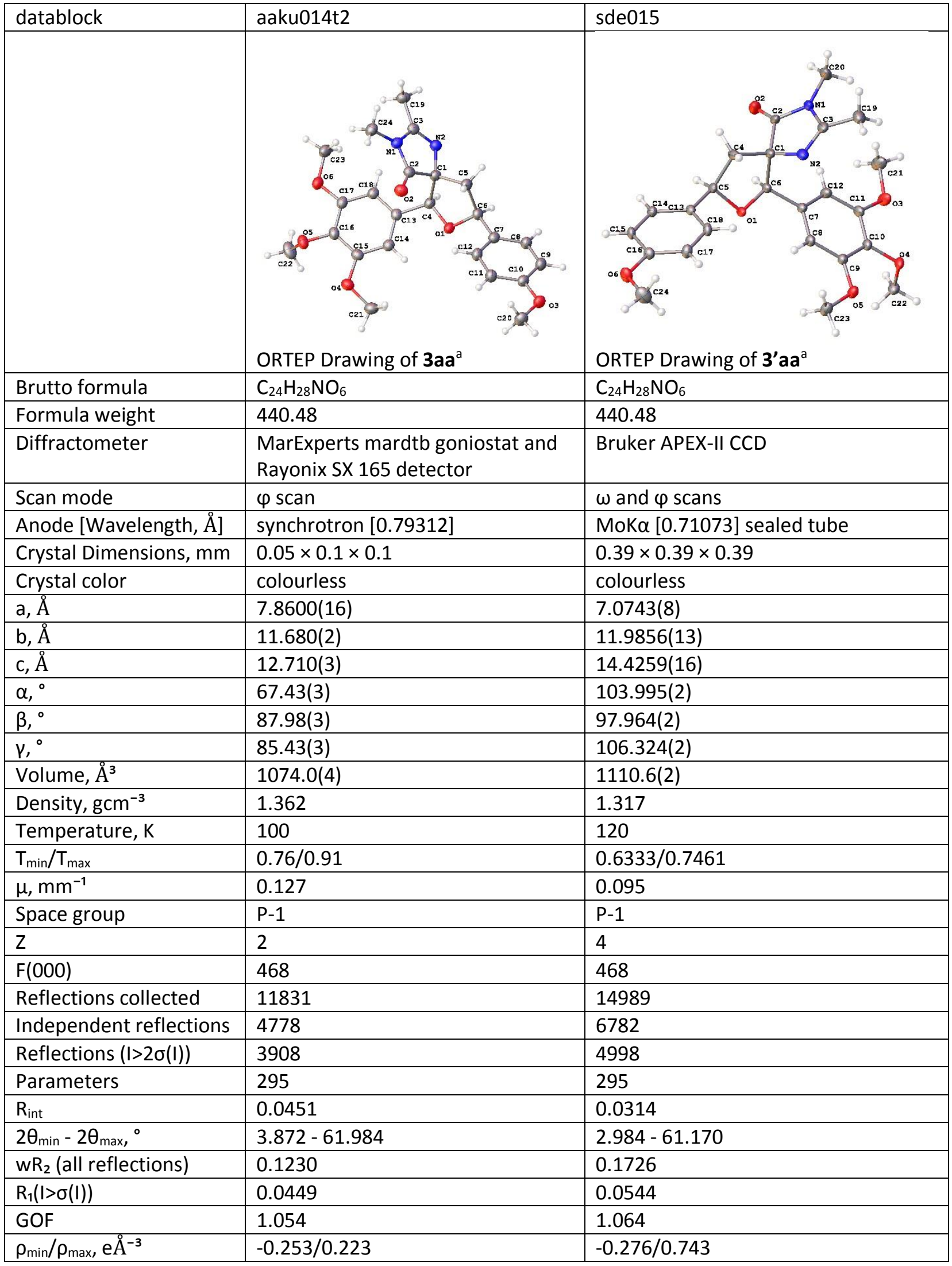

a the thermal ellipsoids are shown at 50\% probability levels 


\section{References}

(1) Reissig, H.-U. Lewis-Acid-Promoted Additions of Carbonyl Compounds to Donor-Acceptor Substituted Cyclopropanes: A New Synthesis of 2,3-Dihydrofurane Derivatives. Tetrahedron Lett. 1981, 22 (31), 2981-2984. https://doi.org/10.1016/S0040-4039(01)81805-0.

(2) Yamago, S.; Nakamura, E. Thermal Hetero [3 + 2] Cycloaddition Approach to Functionalized Tetrahydrofurans. J. Org. Chem. 1990, 55 (21), 5553-5555.

https://doi.org/10.1021/jo00308a006.

(3) Nakamura, I.; Oh, B. H.; Saito, S.; Yamamoto, Y. Novel [3+2] Cycloaddition of Alkylidenecyclopropanes with Aldehydes Catalyzed by Palladium. Angew. Chemie 2001, 113 (7), 1338-1340. https://doi.org/10.1002/1521-3757(20010401)113:7<1338::aid-ange1338>3.0.co;2n.

(4) Christie, S. D. R.; Davoile, R. J.; Elsegood, M. R. J.; Fryatt, R.; Jones, R. C. F.; Pritchard, G. J. Novel Formation and Use of a Nicholas Carbocation in the Synthesis of Highly Substituted

Tetrahydrofurans. Chem. Commun. 2004, 10 (21), 2474-2475. https://doi.org/10.1039/b411367e.

(5) Pohlhaus, P. D.; Johnson, J. S. Highly Diastereoselective Synthesis of Tetrahydrofurans via Lewis Acid-Catalyzed Cyclopropane/Aldehyde Cycloadditions. J. Org. Chem. 2005, 70 (3), 1057-1059. https://doi.org/10.1021/jo048230+.

(6) Pohlhaus, P. D.; Johnson, J. S. Enantiospecific Sn(II)- and Sn(IV)-Catalyzed Cycloadditions of Aldehydes and Donor-Acceptor Cyclopropanes. J. Am. Chem. Soc. 2005, 127 (46), 16014-16015. https://doi.org/10.1021/ja055777c.

(7) Gupta, A.; Yadav, V. K. A Highly Diastereoselective Approach to Tetrahydrofurans via [3+2] Cycloadditions of Silylmethyl-Substituted Cyclopropanes with Aldehydes and Ketones.

Tetrahedron Lett. 2006, 47 (46), 8043-8047. https://doi.org/10.1016/j.tetlet.2006.09.064.

(8) Pohlhaus, P. D.; Sanders, S. D.; Parsons, A. T.; Li, W.; Johnson, J. S. Scope and Mechanism for Lewis Acid-Catalyzed Cycloadditions of Aldehydes and Donor-Acceptor Cyclopropanes: Evidence for a Stereospecific Intimate Ion Pair Pathway. J. Am. Chem. Soc. 2008, 130 (27), 8642-8650. https://doi.org/10.1021/ja8015928.

(9) Parsons, A. T.; Campbell, M. J.; Johnson, J. S. Diastereoselective Synthesis of Tetrahydrofurans via Palladium(0)-Catalyzed [3 + 2] Cycloaddition of Vinylcyclopropanes and Aldehydes. Org. Lett. 2008, 10 (12), 2541-2544. https://doi.org/10.1021/ol800819h.

(10) Parsons, A. T.; Johnson, J. S. Catalytic Enantioselective Synthesis of Tetrahydrofurans: A Dynamic Kinetic Asymmetric [3 + 2] Cycloaddition of Racemic Cyclopropanes and Aldehydes. J. Am. Chem. Soc. 2009, 131 (9), 3122-3123. https://doi.org/10.1021/ja809873u.

(11) Christie, S. D. R.; Cummins, J.; Elsegood, M. R. J.; Dawson, G. Formation of Polysubstituted Tetrahydrofurans on an Iron Tricarbonyl H5-Pentadienyl Template. Synlett 2009, No. 2, 257-259. https://doi.org/10.1055/s-0028-1087516.

(12) Xing, S.; Pan, W.; Liu, C.; Ren, J.; Wang, Z. Efficient Construction of Oxa- and Aza-[n.2.1] Skeletons: Lewis Acid Catalyzed Intramolecular [3+2] Cycloaddition of Cyclopropane 1,1-Diesters with Carbonyls and Imines. Angew. Chemie - Int. Ed. 2010, 49 (18), 3215-3218. https://doi.org/10.1002/anie.201000563.

(13) Smith, A. G.; Slade, M. C.; Johnson, J. S. Cyclopropane-Aldehyde Annulations at Quaternary Donor Sites: Stereoselective Access to Highly Substituted Tetrahydrofurans. Org. Lett. 2011, 13 (8), 1996-1999. https://doi.org/10.1021/ol200395e.

(14) Yang, G.; Shen, Y.; Li, K.; Sun, Y.; Hua, Y. AlCl3-Promoted Highly Regio- and Diastereoselective [3 + 
2] Cycloadditions of Activated Cyclopropanes and Aromatic Aldehydes: Construction of 2,5Diaryl-3,3,4-Trisubstituted Tetrahydrofurans. J. Org. Chem. 2011, 76 (1), 229-233. https://doi.org/10.1021/jo1020773.

(15) Fujino, D.; Yorimitsu, H.; Oshima, K. Synthesis of Alkylidenecyclopropanes by Palladium-Catalyzed Reaction of Propargyl-Substituted Malonate Esters with Aryl Halides by Anti-Carbopalladation Pathway. J. Am. Chem. Soc. 2011, 133 (25), 9682-9685. https://doi.org/10.1021/ja203062z.

(16) Xu, X.; Lu, H.; Ruppel, J. V; Cui, X.; Lopez de Mesa, S.; Wojtas, L.; Zhang, X. P. Highly Asymmetric Intramolecular Cyclopropanation of Acceptor-Substituted Diazoacetates by Co(II)-Based Metalloradical Catalysis: Iterative Approach for Development of New-Generation Catalysts. J. Am. Chem. Soc. 2011, 133 (39), 15292-15295. https://doi.org/10.1021/ja2062506.

(17) Benfatti, F.; Nanteuil, F. De; Waser, J. Iron-Catalyzed [3 + 2] Annulation of Aminocyclopropanes with Aldehydes: Stereoselective Synthesis of Aminotetrahydrofurans. Org. Lett. 2012, 14 (1), 386-389. https://doi.org/10.1021/ol203144v.

(18) Benfatti, F.; De Nanteuil, F.; Waser, J. Catalytic Enantiospecific [3+2] Annulation of Aminocyclopropanes with Ketones. Chem. - A Eur. J. 2012, 18 (16), 4844-4849. https://doi.org/10.1002/chem.201103971.

(19) Bai, Y.; Tao, W.; Ren, J.; Wang, Z. Lewis Acid Catalyzed Intramolecular [4+2] and [3+2] CrossCycloaddition of Alkynylcyclopropane Ketones with Carbonyl Compounds and Imines. Angew. Chemie - Int. Ed. 2012, 51 (17), 4112-4116. https://doi.org/10.1002/anie.201200450.

(20) Nani, R. R.; Reisman, S. E. $\alpha$-Diazo- $\beta$-Ketonitriles: Uniquely Reactive Substrates for Arene and Alkene Cyclopropanation. J. Am. Chem. Soc. 2013, 135 (19), 7304-7311. https://doi.org/10.1021/ja401610p.

(21) Miyake, Y.; Endo, S.; Moriyama, T.; Sakata, K.; Nishibayashi, Y. Ruthenium-Triggered Ring Opening of Ethynylcyclopropanes: [3+2] Cycloaddition with Aldehydes and Aldimines Involving Metal Allenylidene Intermediates. Angew. Chemie - Int. Ed. 2013, 52 (6), 1758-1762. https://doi.org/10.1002/anie.201207801.

(22) Haubenreisser, S.; Hensenne, P.; Schröder, S.; Niggemann, M. The Alkynyl Moiety as a Donor for Donor-Acceptor Cyclopropanes. Org. Lett. 2013, 15 (9), 2262-2265. https://doi.org/10.1021/ol400809n.

(23) Cao, Z. Y.; Zhou, F.; Yu, Y. H.; Zhou, J. A Highly Diastereo- and Enantioselective Hg(II)-Catalyzed Cyclopropanation of Diazooxindoles and Alkenes. Org. Lett. 2013, 15 (1), 42-45. https://doi.org/10.1021/ol302998m.

(24) Hung, H. H.; Liao, Y. C.; Liu, R. S. Silver-Catalyzed Stereoselective [3+2] Cycloadditions of Cyclopropyl-Indanimines with Carbonyl Compounds. Adv. Synth. Catal. 2013, 355 (8), 1545-1552. https://doi.org/10.1002/adsc.201300090.

(25) Ma, X.; Tang, Q.; Ke, J.; Yang, X.; Zhang, J.; Shao, H. InCl3 Catalyzed Highly Diastereoselective [3 + 2] Cycloaddition of 1,2-Cyclopropanated Sugars with Aldehydes: A Straightforward Synthesis of Persubstituted Bis -Tetrahydrofurans and Perhydrofuro[2,3- b ]Pyrans. Org. Lett. 2013, 15 (20), 5170-5173. https://doi.org/10.1021/ol402192f.

(26) De Nanteuil, F.; Serrano, E.; Perrotta, D.; Waser, J. Dynamic Kinetic Asymmetric [3 + 2] Annulation Reactions of Aminocyclopropanes. J. Am. Chem. Soc. 2014, 136 (17), 6239-6242. https://doi.org/10.1021/ja5024578.

(27) Racine, S.; Denanteuil, F.; Serrano, E.; Waser, J. Synthesis of (Carbo)Nucleoside Analogues by [3+2] Annulation of Aminocyclopropanes. Angew. Chemie - Int. Ed. 2014, 53 (32), 8484-8487. https://doi.org/10.1002/anie.201404832. 
(28) Wang, L. F.; Shi, Z. F.; Cao, X. P.; Li, B. S.; An, P. Construction of Fused- and Spiro-Oxa-[n.2.1] Skeletons by a Tandem Epoxide Rearrangement/Intramolecular [3+2] Cycloaddition of Cyclopropanes with Carbonyls. Chem. Commun. 2014, 50 (59), 8061-8064. https://doi.org/10.1039/c4cc02641a.

(29) Shiba, T.; Kuroda, D.; Kurahashi, T.; Matsubara, S. Cobalt Porphyrin Catalyzed [3+2] Cycloaddition of Cyclopropanes and Carbonyl Compounds. Synlett 2014, 25 (14), 2005-2008. https://doi.org/10.1055/s-0034-1378394.

(30) Ren, J.; Bao, J.; Ma, W.; Wang, Z. Tandem Diels-Alder [4+2] Cycloadditions and Intramolecular [3+2] Cross-Cycloadditions of Dienylcyclopropane 1,1-Diesters. Synlett 2014, 25 (16), 2260-2264. https://doi.org/10.1055/s-0034-1378897.

(31) Zhang, J.; Xing, S.; Ren, J.; Jiang, S.; Wang, Z. Lewis Acid Catalyzed Intramolecular [3 + 2] Cross Cycloadditions of Cobalt-Alkynylcyclopropane 1,1-Diesters with Carbonyls for Construction of Medium-Sized and Polycyclic Skeletons. Org. Lett. 2015, 17 (2), 218-221. https://doi.org/10.1021/ol503285u.

(32) Wang, Z.; Chen, S.; Ren, J.; Wang, Z. Cooperative Photo-/Lewis Acid Catalyzed Tandem Intramolecular [3+2] Cross-Cycloadditions of Cyclopropane 1,1-Diesters with $\alpha, \beta$-Unsaturated Carbonyls for Medium-Sized Carbocycles. Org. Lett. 2015, 17 (17), 4184-4187. https://doi.org/10.1021/acs.orglett.5b01928.

(33) Klimczyk, S.; Misale, A.; Huang, X.; Maulide, N. Dimeric TADDOL Phosphoramidites in Asymmetric Catalysis: Domino Deracemization and Cyclopropanation of Sulfonium Ylides. Angew. Chemie Int. Ed. 2015, 54 (35), 10365-10369. https://doi.org/10.1002/anie.201503851.

(34) Sabbatani, J.; Maulide, N. Temporary Generation of a Cyclopropyl Oxocarbenium Ion Enables Highly Diastereoselective Donor-Acceptor Cyclopropane Cycloaddition. Angew. Chemie - Int. Ed. 2016, 55 (23), 6780-6783. https://doi.org/10.1002/anie.201601340.

(35) Zhang, C.; Xu, M.; Ren, J.; Wang, Z. Sc(OTf)3-Catalyzed Diastereoselective Formal [3+2] Cycloaddition Reactions of Alkynylcyclopropane Ketones with Electron-Rich Aromatic Aldehydes to Yield 2,5-Trans-Tetrahydrofurans. European J. Org. Chem. 2016, 2016 (14), 2467-2478. https://doi.org/10.1002/ejoc.201600233.

(36) Xiao, J. A.; Li, J.; Xia, P. J.; Zhou, Z. F.; Deng, Z. X.; Xiang, H. Y.; Chen, X. Q.; Yang, H. Diastereoselective Intramolecular [3 + 2]-Annulation of Donor-Acceptor Cyclopropane with Imine-Assembling Hexahydropyrrolo[3,2-c]Quinolinone Scaffolds. J. Org. Chem. 2016, 81 (22), 11185-11194. https://doi.org/10.1021/acs.joc.6b02172.

(37) Gupta, A.; Kholiya, R.; Rawat, D. S. BF3. OEt2-Mediated Highly Stereoselective Synthesis of Trisubstituted-Tetrahydrofuran via [3+2] Cycloaddition Reaction of 2-Arylcyclopropyl Ketones with Aldehydes. Asian J. Org. Chem. 2017, 6 (8), 993-997. https://doi.org/10.1002/ajoc.201700244.

(38) Xu, P. W.; Liu, J. K.; Shen, L.; Cao, Z. Y.; Zhao, X. L.; Yan, J.; Zhou, J. Diastereo- and Enantioselective [3 + 3] Cycloaddition of Spirocyclopropyl Oxindoles Using Both Aldonitrones and Ketonitrones. Nat. Commun. 2017, 8 (1). https://doi.org/10.1038/s41467-017-01451-1.

(39) Xu, H.; Li, Y. P.; Cai, Y.; Wang, G. P.; Zhu, S. F.; Zhou, Q. L. Highly Enantioselective Copper-and Iron-Catalyzed Intramolecular Cyclopropanation of Indoles. J. Am. Chem. Soc. 2017, 139 (23), 7697-7700. https://doi.org/10.1021/jacs.7b03086.

(40) Tang, Y.; Yu, Q.; Ma, S. Efficient Trifluoromethylation: Via the Cyclopropanation of Allenes and Subsequent C-C Bond Cleavage. Org. Chem. Front. 2017, 4 (9), 1762-1767. https://doi.org/10.1039/c7qo00419b.

(41) Kreft, A.; Jones, P. G.; Werz, D. B. The Cyclopropyl Group as a Neglected Donor in Donor-Acceptor 
Cyclopropane Chemistry. Org. Lett. 2018, 20 (7), 2059-2062.

https://doi.org/10.1021/acs.orglett.8b00603.

(42) Braun, J.; Ariëns, M. I.; Matsuo, B. T.; De Vries, S.; Van Wordragen, E. D. H.; Ellenbroek, B. D.; Vande Velde, C. M. L.; Orru, R. V. A.; Ruijter, E. Stereoselective Synthesis of Fused Vinylcyclopropanes by Intramolecular Tsuji-Trost Cascade Cyclization. Org. Lett. 2018, 20 (21), 6611-6615. https://doi.org/10.1021/acs.orglett.8b02232.

(43) Shen, Y.; Yang, P. F.; Yang, G.; Chen, W. L.; Chai, Z. Lewis Acid-Catalyzed Enantiospecific [3 + 2] Annulations of $\gamma$-Butyrolactone Fused Cyclopropanes with Aromatic Aldehydes: Synthesis of Chiral Furanolignans. Org. Biomol. Chem. 2018, 16 (15), 2688-2696.

https://doi.org/10.1039/c8ob00455b.

(44) Yang, P.; Shen, Y.; Feng, M.; Yang, G.; Chai, Z. Lewis Acid Catalyzed [3+2] Annulation of YButyrolactone Fused Cyclopropane with Aldehydes/Ketones. European J. Org. Chem. 2018, 2018 (30), 4103-4112. https://doi.org/10.1002/ejoc.201800695.

(45) Shen, Y.; Chai, J.; Yang, G.; Chen, W.; Chai, Z. Stereocontrolled Synthesis of Trans/ Cis-2,3Disubstituted Cyclopropane-1,1-Diesters and Applications in the Syntheses of Furanolignans. J. Org. Chem. 2018, 83 (20), 12549-12558. https://doi.org/10.1021/acs.joc.8b01798.

(46) Baranov, M. S.; Solntsev, K. M.; Lukyanov, K. A.; Yampolsky, I. V. A Synthetic Approach to GFP Chromophore Analogs from 3-Azidocinnamates. Role of Methyl Rotors in Chromophore Photophysics. Chem. Commun. 2013, 49 (51), 5778-5780. https://doi.org/10.1039/c3cc41948g.

(47) Povarova, N. V.; Zaitseva, S. O.; Baleeva, N. S.; Smirnov, A. Y.; Myasnyanko, I. N.; Zagudaylova, M. B.; Bozhanova, N. G.; Gorbachev, D. A.; Malyshevskaya, K. K.; Gavrikov, A. S.; et al. Red-Shifted Substrates for FAST Fluorogen-Activating Protein Based on the GFP-Like Chromophores. Chem. A Eur. J. 2019, 25 (41), 9592-9596. https://doi.org/10.1002/chem.201901151.

(48) Schmidt, E. Über Alkylierte Iso-[Acyl-glykokollester] Und Phenylhydrazidoester. Berichte der Dtsch. Chem. Gesellschaft 1914. https://doi.org/10.1002/cber.19140470327.

(49) Williams, S. J.; Zammit, S. C.; Cox, A. J.; Shackleford, D. M.; Morizzi, J.; Zhang, Y.; Powell, A. K.; Gilbert, R. E.; Krum, H.; Kelly, D. J. 3',4'-Bis-Difluoromethoxycinnamoylanthranilate (FT061): An Orally-Active Antifibrotic Agent That Reduces Albuminuria in a Rat Model of Progressive Diabetic Nephropathy. Bioorganic Med. Chem. Lett. 2013, 23 (24), 6868-6873. https://doi.org/10.1016/j.bmcl.2013.09.100.

(50) Goldberg, A. F. G.; O'Connor, N. R.; Craig, R. A.; Stoltz, B. M. Lewis Acid Mediated (3+2) Cycloadditions of Donor-Acceptor Cyclopropanes with Heterocumulenes. Org. Lett. 2012. https://doi.org/10.1021/ol302494n.

(51) Tiemann, F. Ueber Die Mononitrirten Oxybenzaldehyde Und Ihre Methyläther. Berichte der Dtsch. Chem. Gesellschaft 1889. https://doi.org/10.1002/cber.188902202113.

(52) Sheldrick, G. M. SHELXT - Integrated Space-Group and Crystal-Structure Determination. Acta Crystallogr. Sect. A Found. Adv. 2015, 71 (1), 3-8. https://doi.org/10.1107/S2053273314026370.

(53) Sheldrick, G. M. Crystal Structure Refinement with SHELXL. Acta Crystallogr. Sect. C Struct. Chem. 2015, 71 (1), 3-8. https://doi.org/10.1107/S2053229614024218.

(54) Dolomanov, O. V.; Bourhis, L. J.; Gildea, R. J.; Howard, J. A. K.; Puschmann, H. OLEX2 : A Complete Structure Solution, Refinement and Analysis Program. J. Appl. Crystallogr. 2009, 42 (2), 339-341. https://doi.org/10.1107/S0021889808042726. 


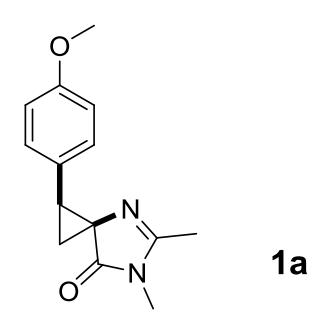

${ }^{1} \mathrm{H}$ NMR (300 MHz), CD $\mathrm{D}_{3} \mathrm{OD}$
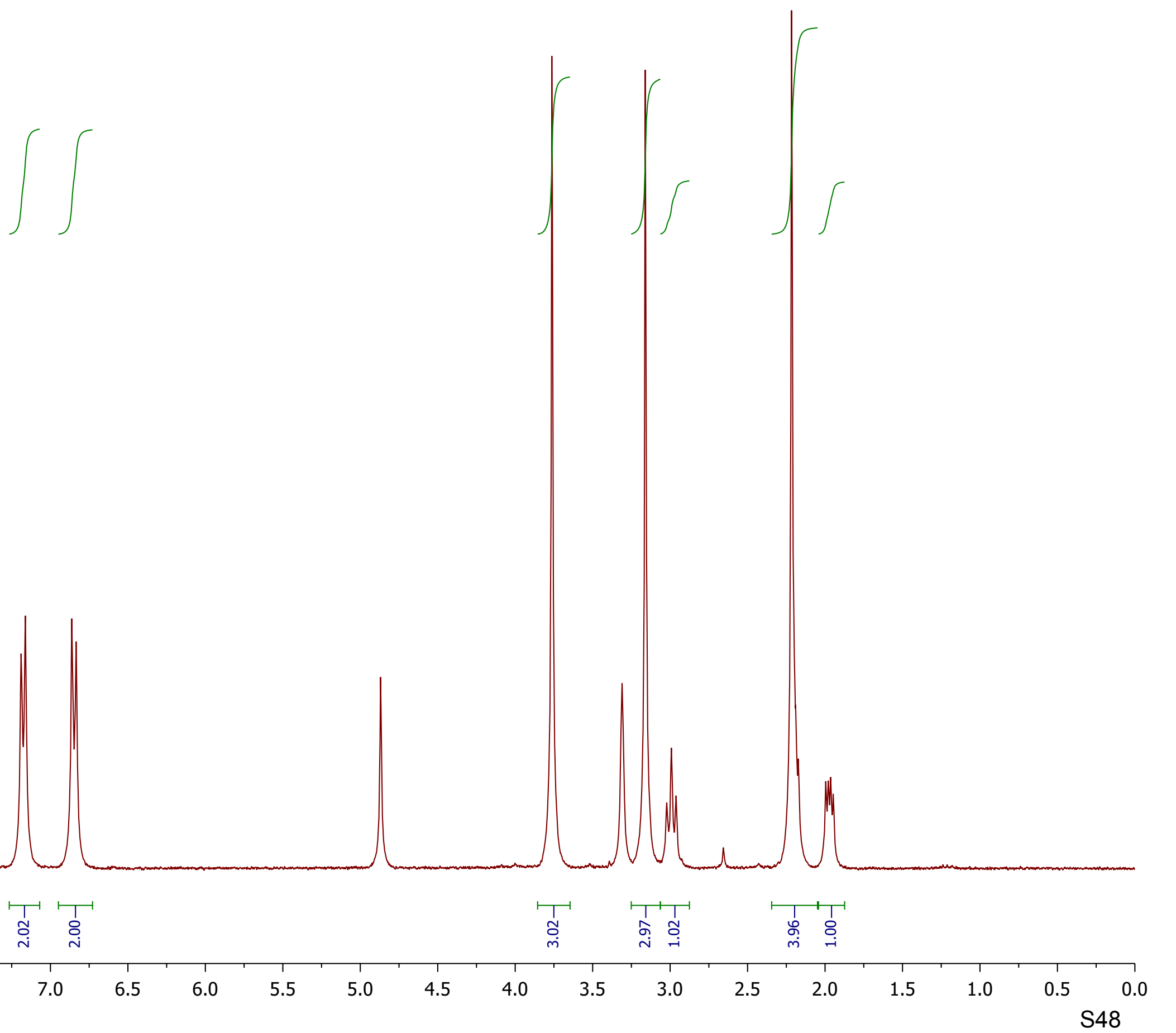


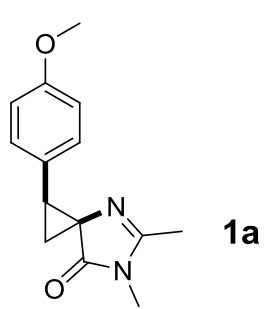

\section{${ }^{13} \mathrm{C}$ NMR (75 MHz), CD $\mathrm{OD}$}

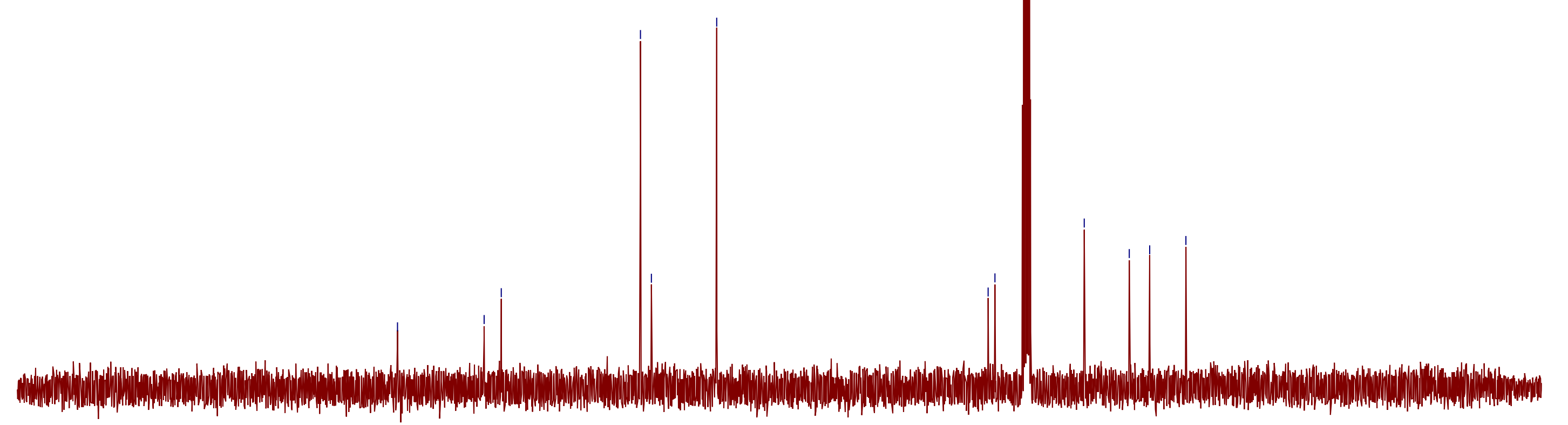

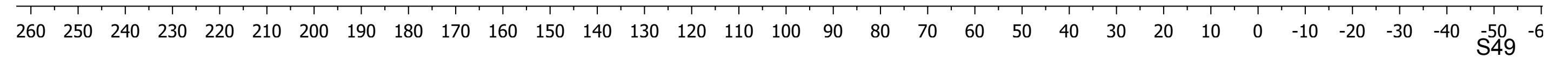


${ }^{1} \mathrm{H}$ NMR (300 MHz), $\mathrm{CDCl}_{3}$
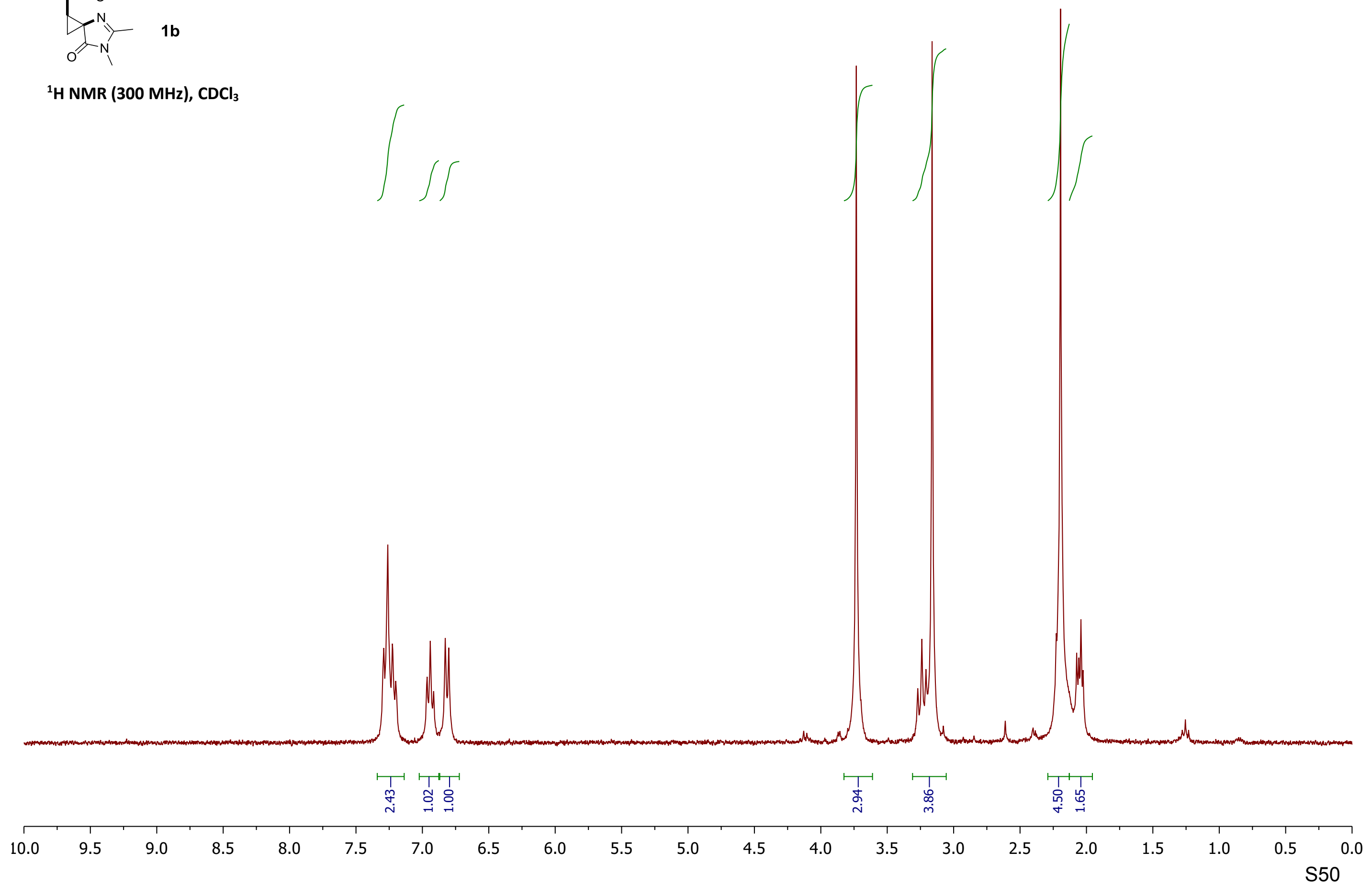


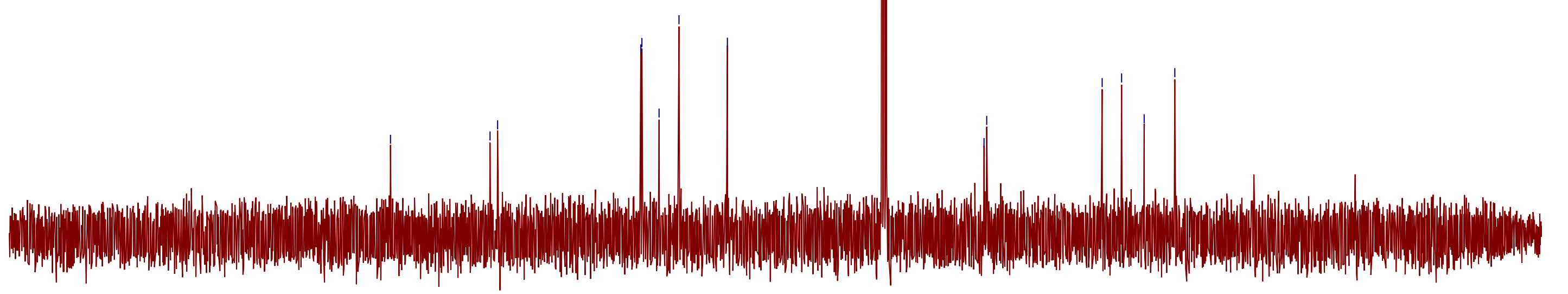

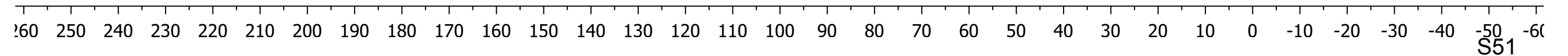




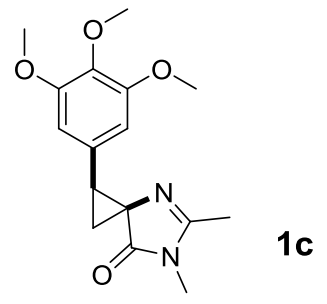

${ }^{1} \mathrm{H}$ NMR (300 MHz), $\mathrm{CDCl}_{3}$

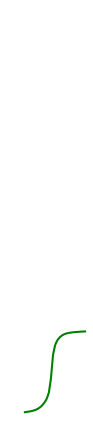

$\int$

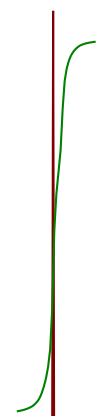

$\int \Gamma$ $\int 11$

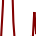

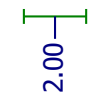
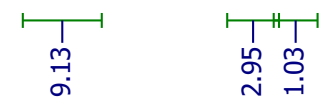

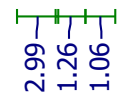

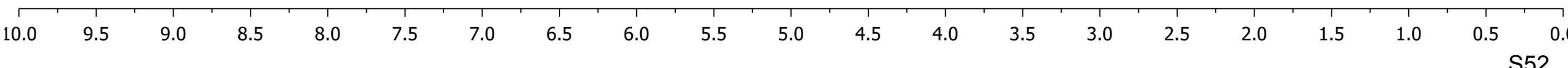




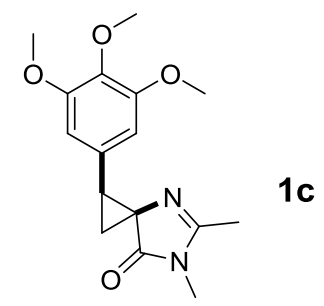

${ }^{13} \mathrm{C} \mathrm{NMR}(75 \mathrm{MHz}), \mathrm{CDCl}_{3}$

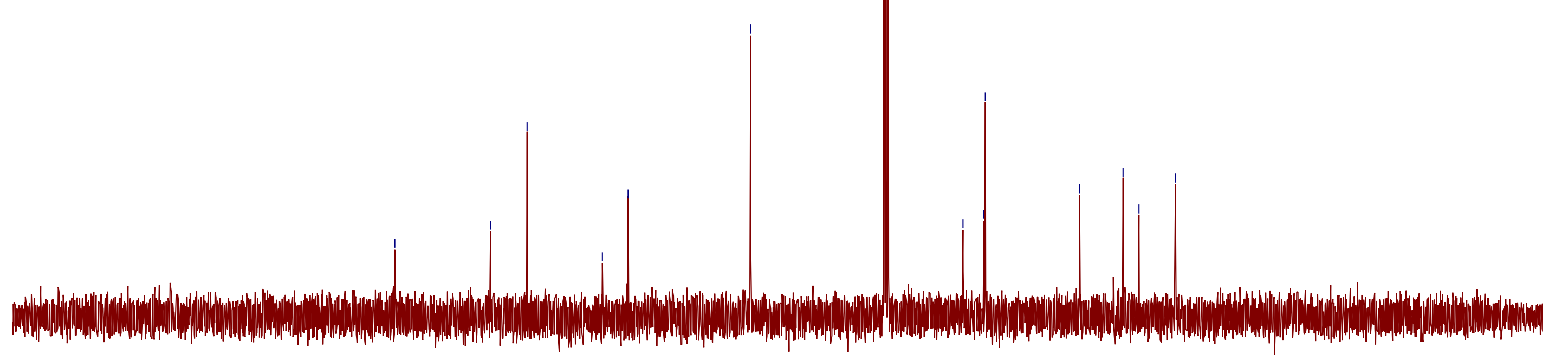

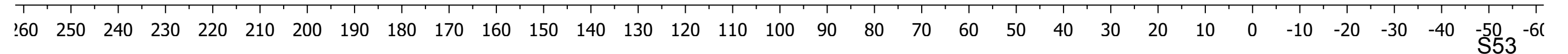




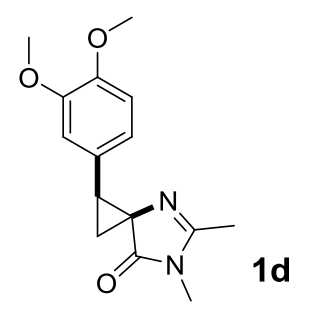

${ }^{1} \mathrm{H}$ NMR (300 MHz), $\mathrm{CDCl}_{3}$
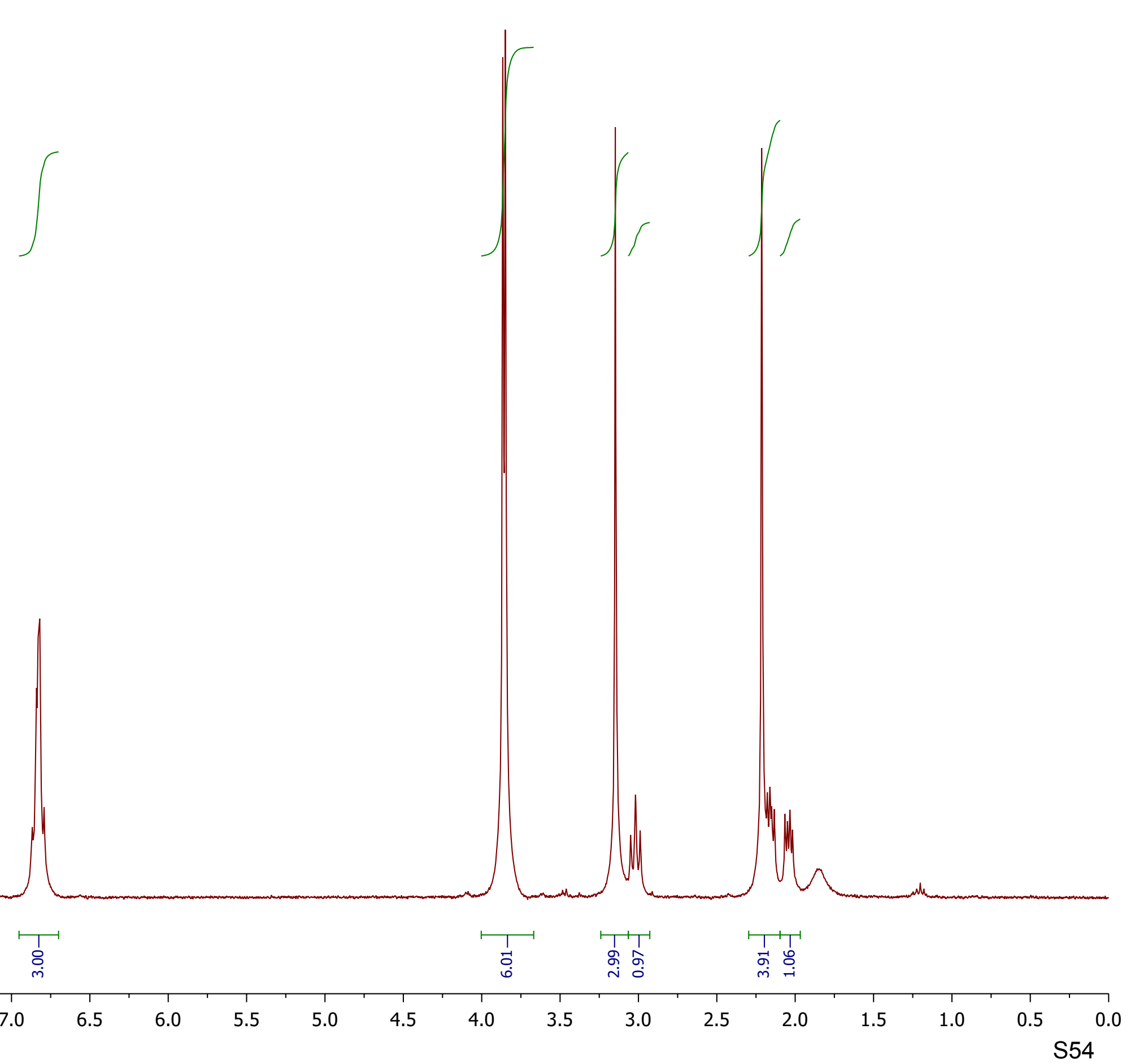


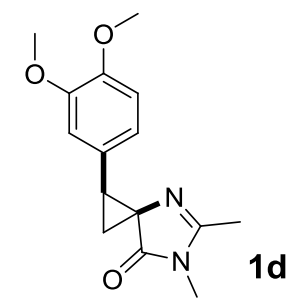

${ }^{13} \mathrm{C} \mathrm{NMR}$ (75 MHz), $\mathrm{CDCl}_{3}$

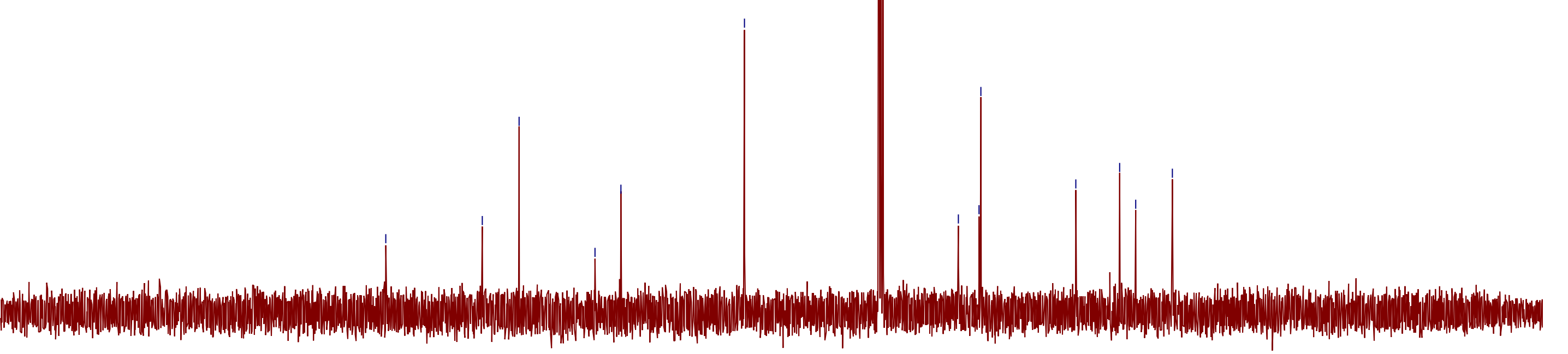

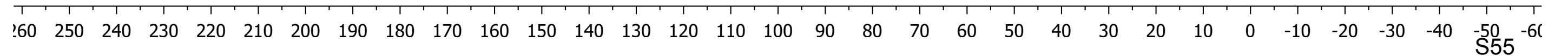




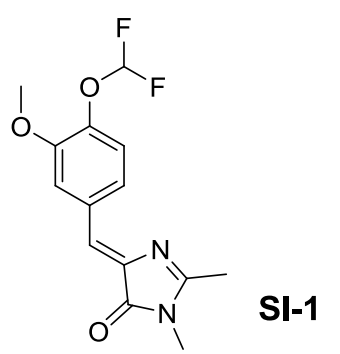

${ }^{1} \mathrm{H}$ NMR (300 MHz), $\mathrm{CDCl}_{3}$
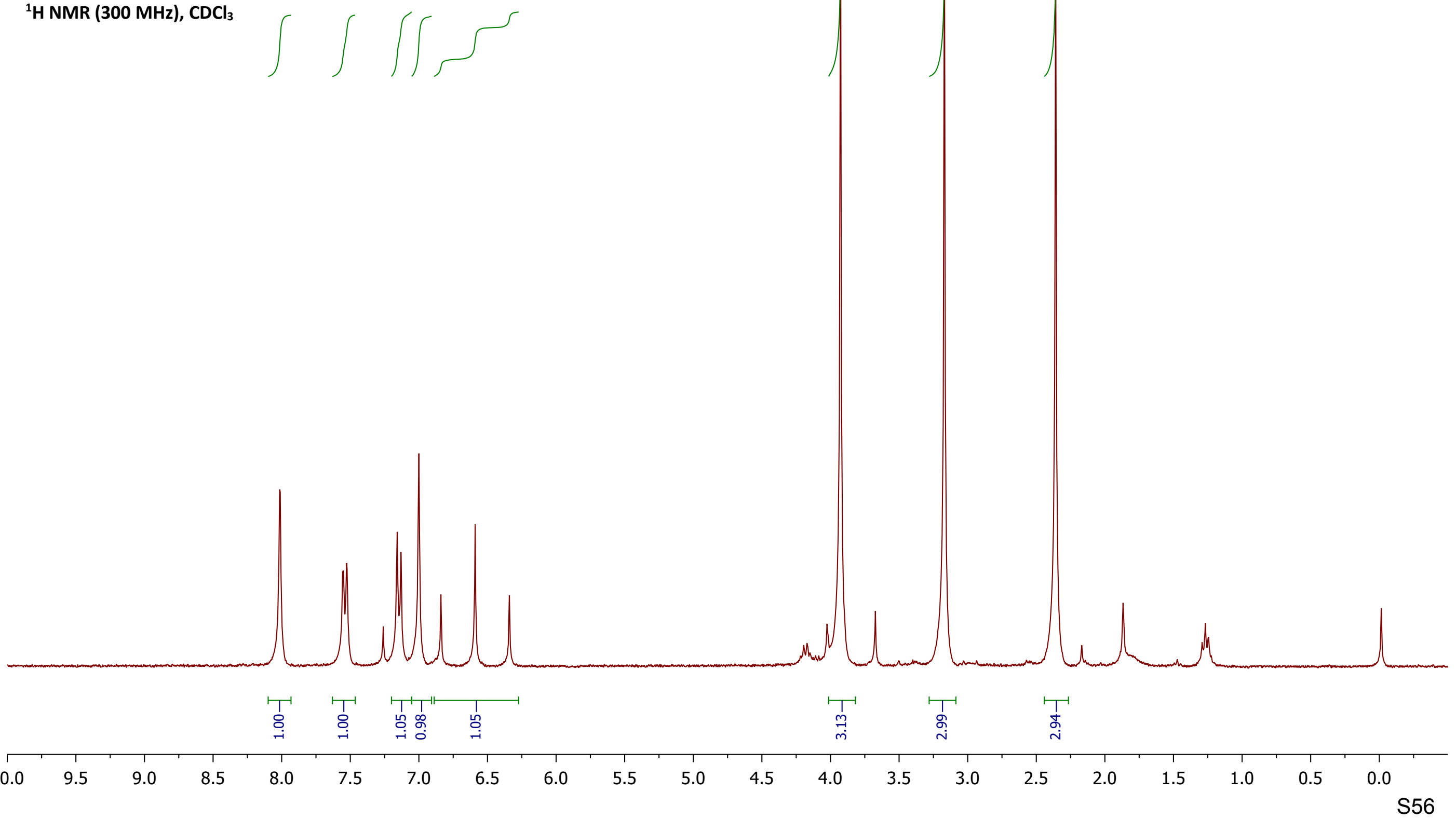


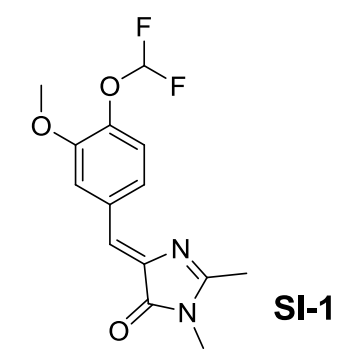

${ }^{13} \mathrm{C}$ NMR (75 MHz), $\mathrm{CDCl}_{3}$

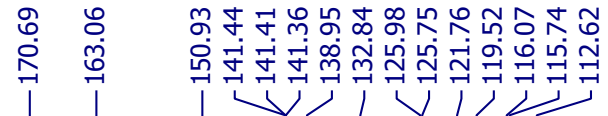

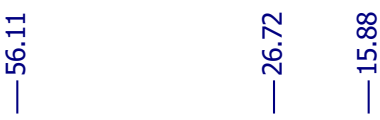

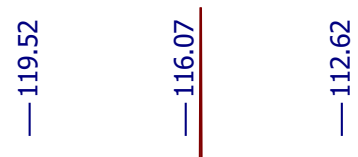

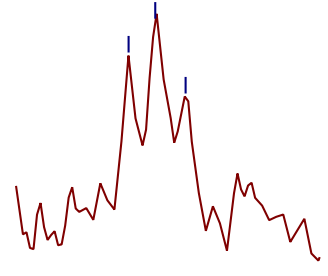

141.6
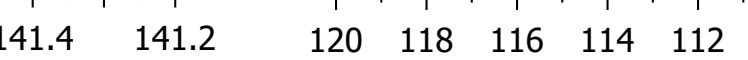

W.y.

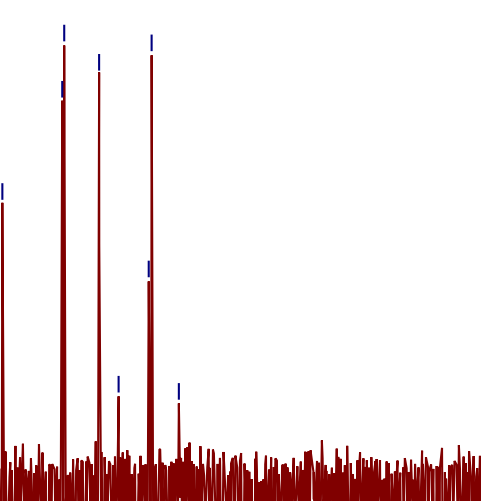




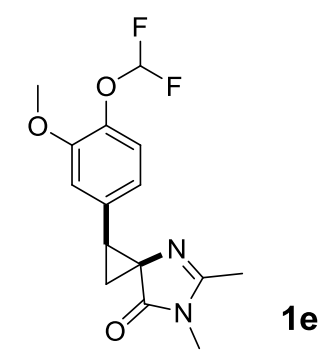

${ }^{1} \mathrm{H}$ NMR (700 MHz), $\mathrm{CDCl}_{3}$
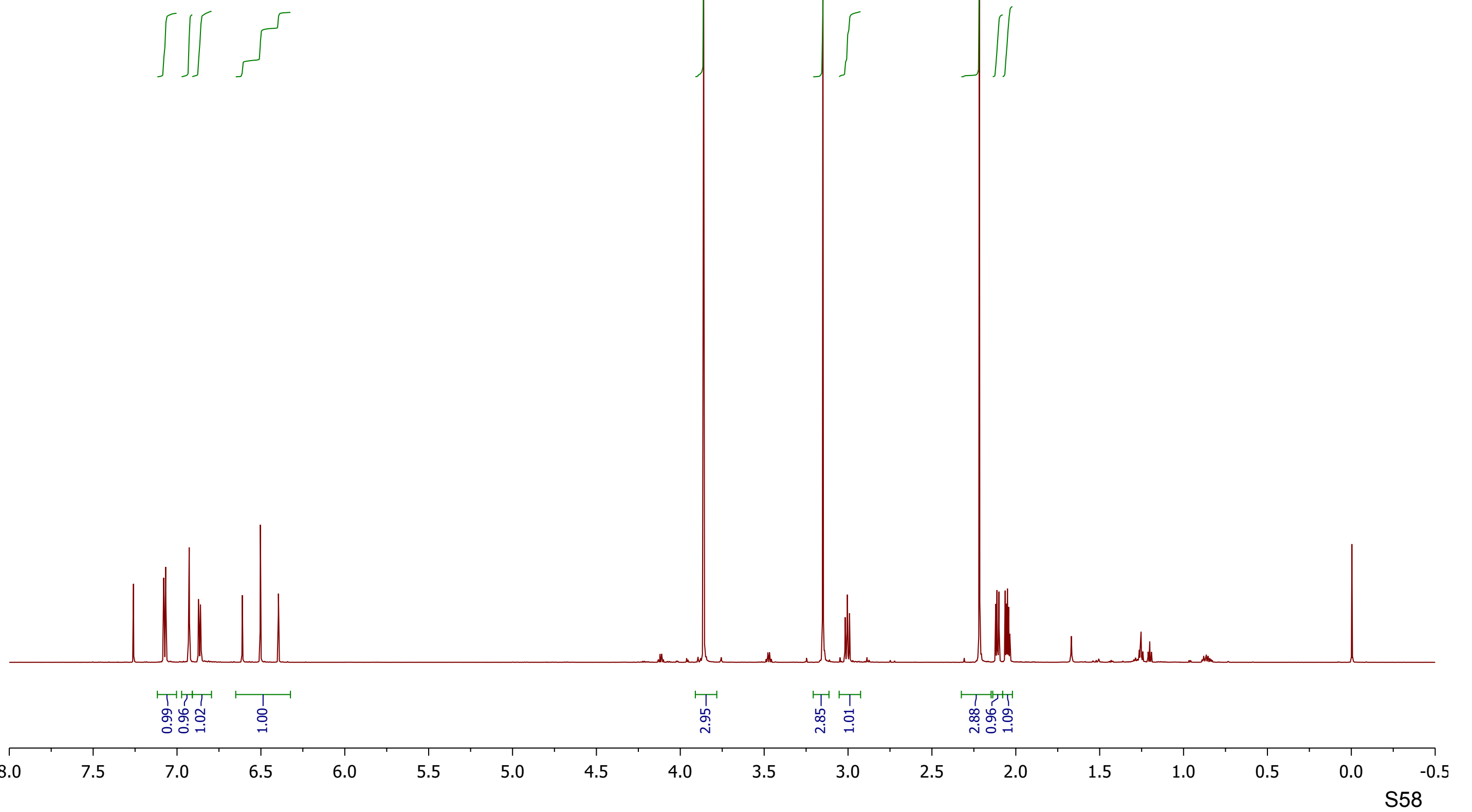


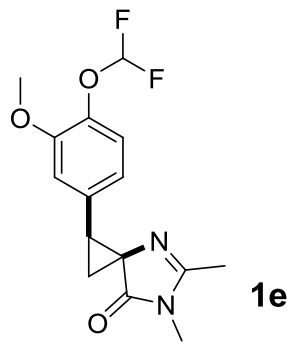

${ }^{13} \mathrm{C}$ NMR (175 MHz), $\mathrm{CDCl}_{3}$

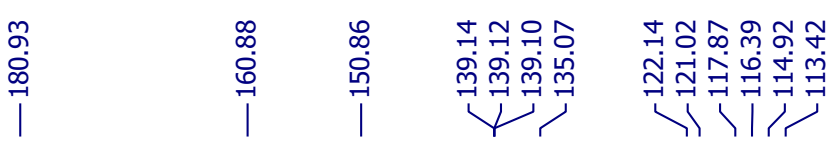

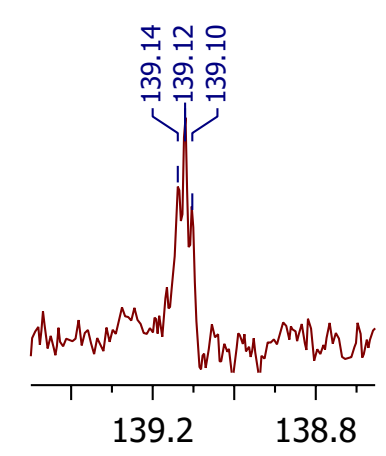

นึ่

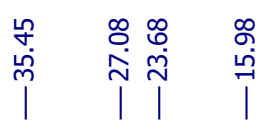
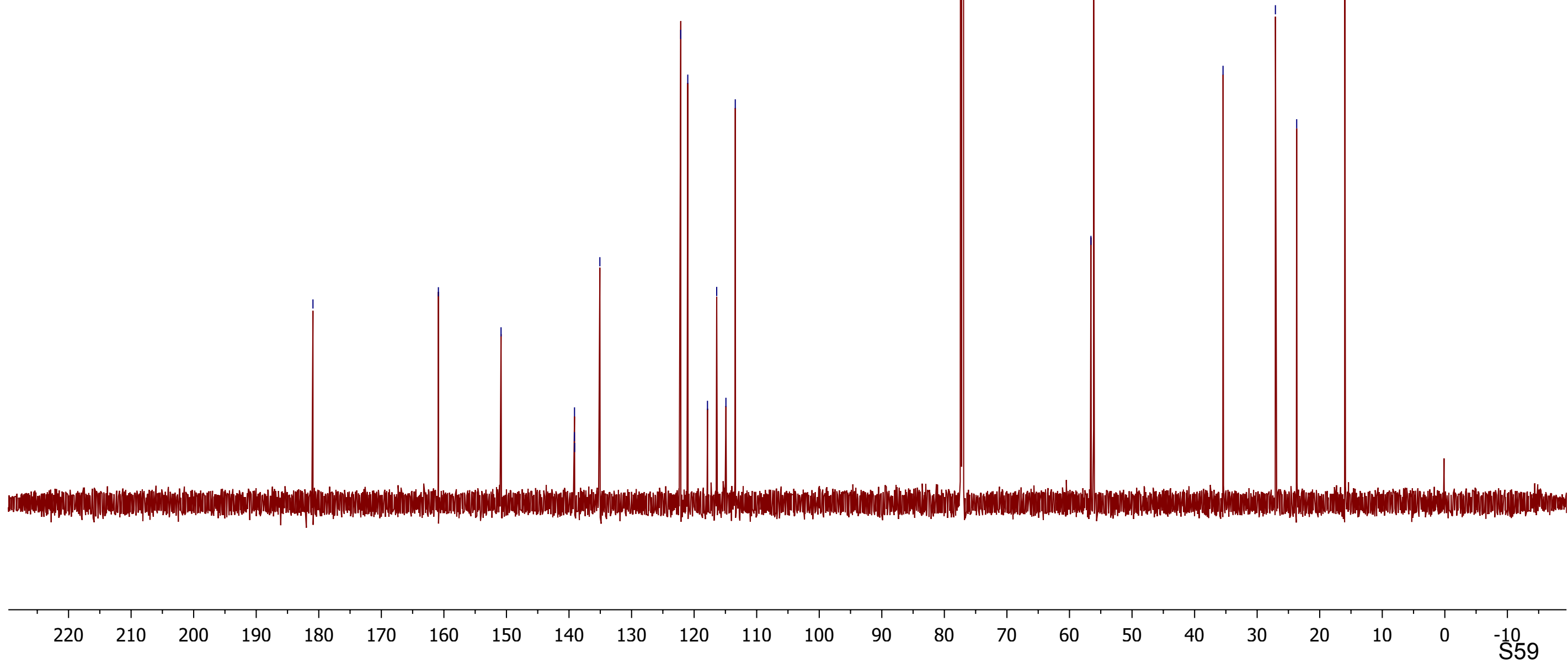
11

${ }^{1} \mathrm{H}$ NMR (300 MHz), $\mathrm{CDCl}_{3}$
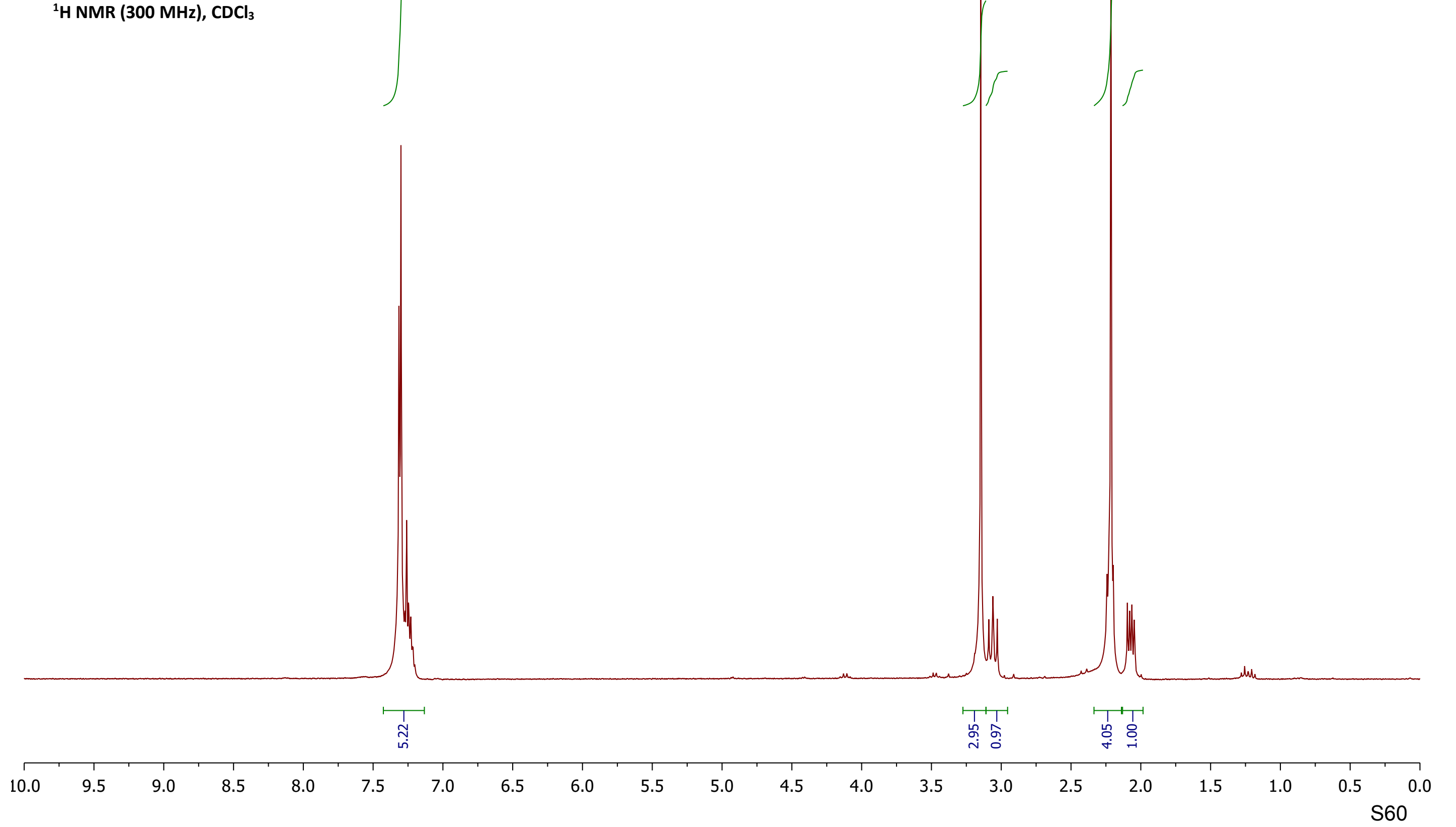


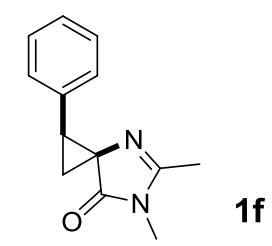

${ }^{13} \mathrm{C}$ NMR (75 MHz), $\mathrm{CDCl}_{3}$

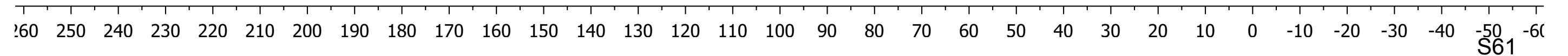




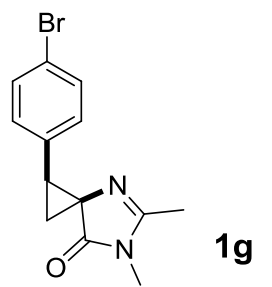

${ }^{1} \mathrm{H}$ NMR (300 MHz), $\mathrm{CDCl}_{3}$
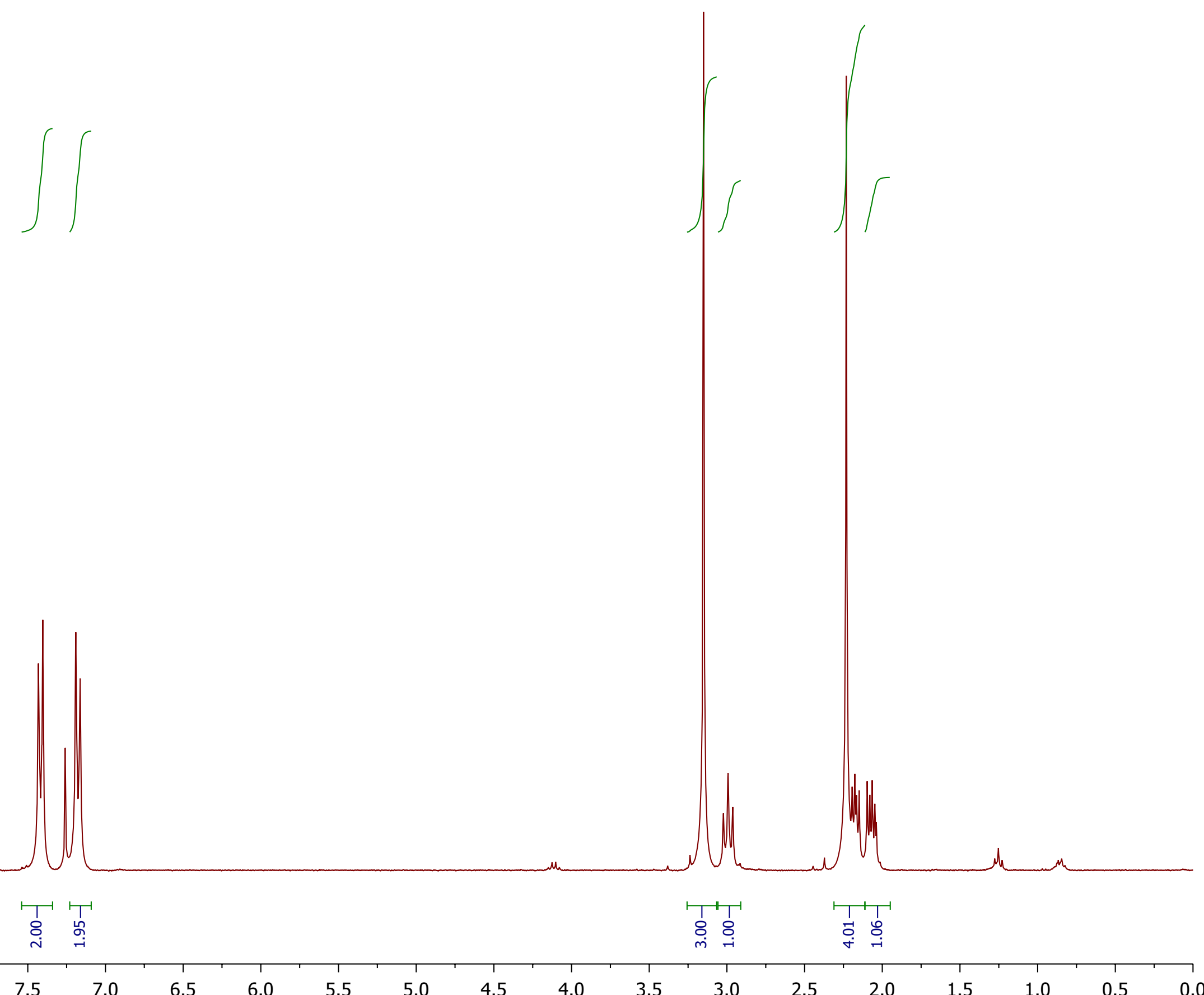


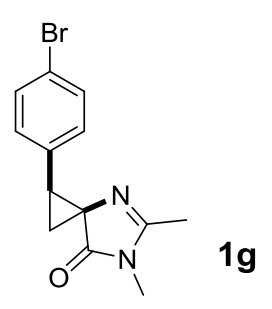

\section{${ }^{13} \mathrm{C}$ NMR (75 MHz), $\mathrm{CDCl}_{3}$}
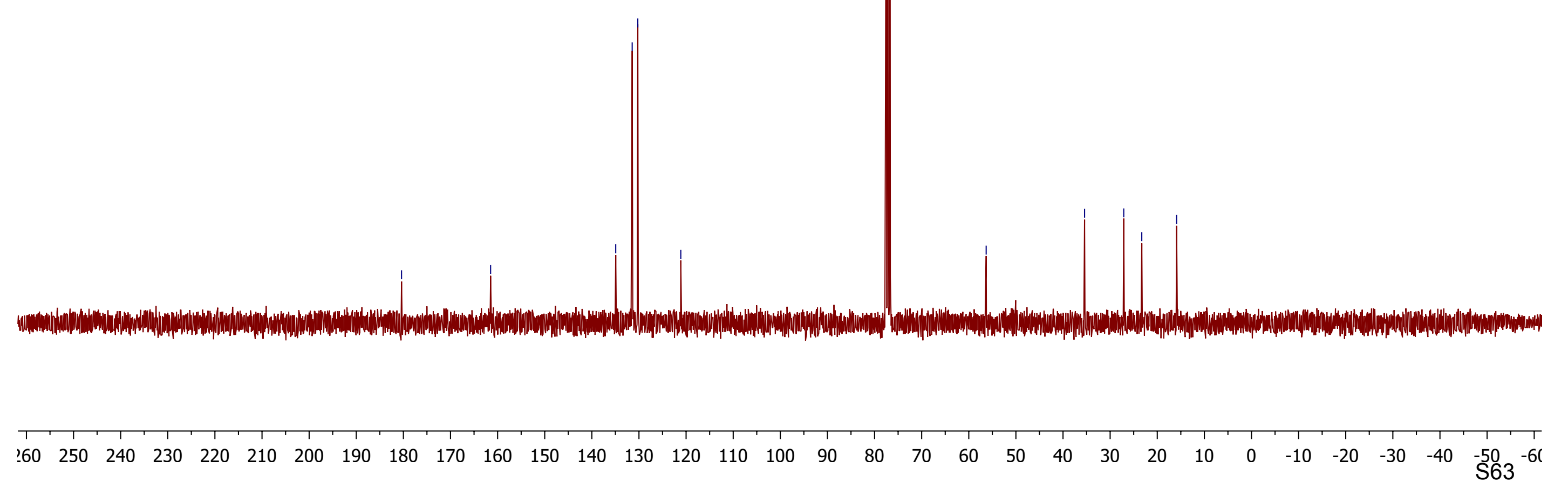
$\mathrm{C}^{S}{ }^{\mathrm{N}} 1 \mathrm{n}$

${ }^{1} \mathrm{H}$ NMR (300 MHz), $\mathrm{CDCl}_{3}$
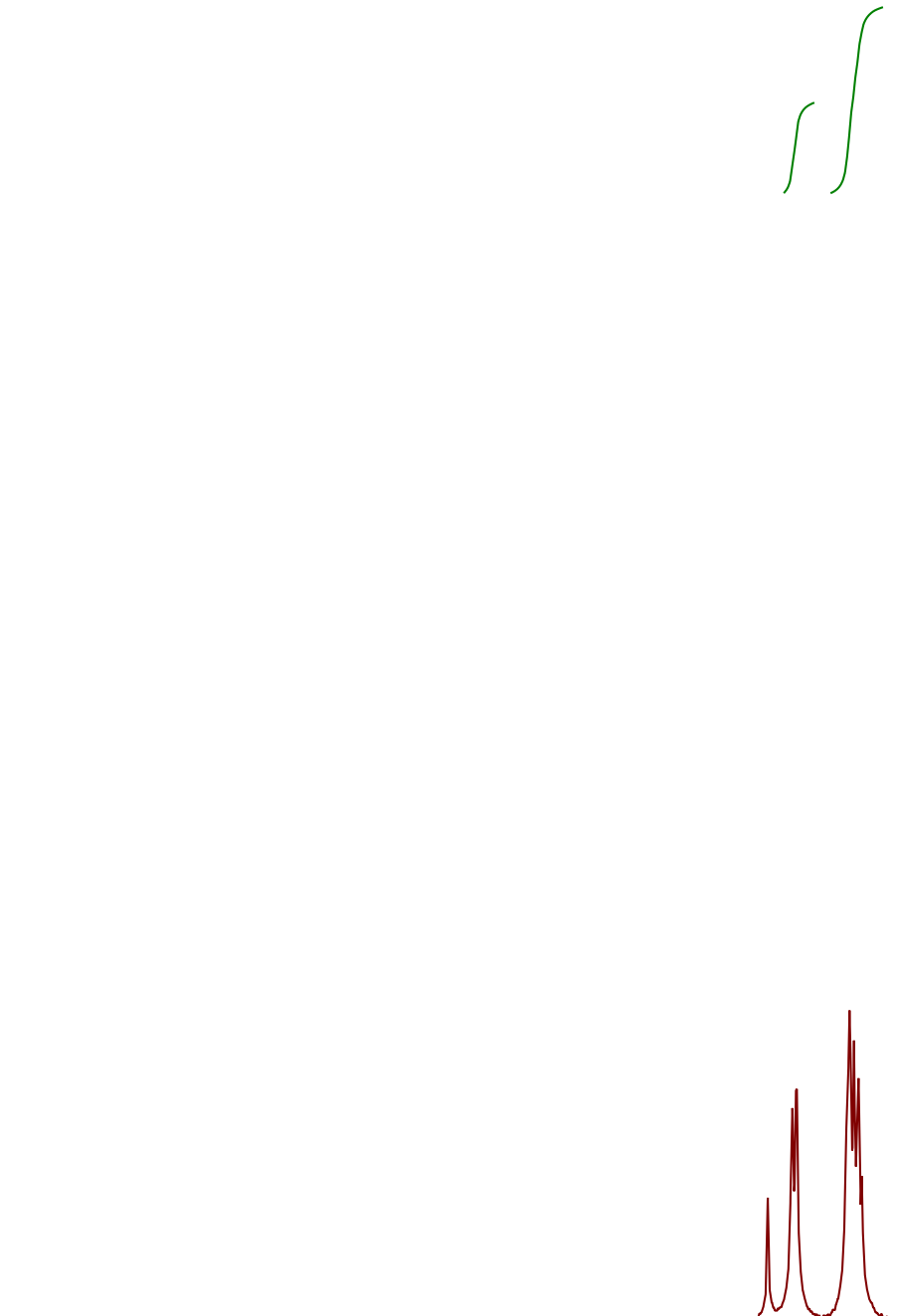

†T

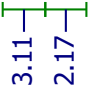




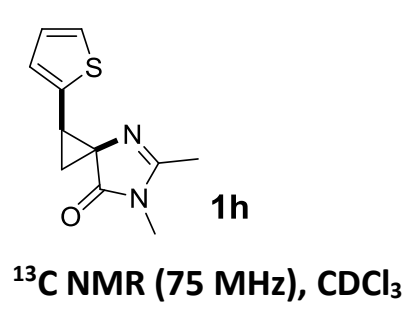

${ }^{13} \mathrm{C}$ NMR (75 MHz), $\mathrm{CDCl}_{3}$

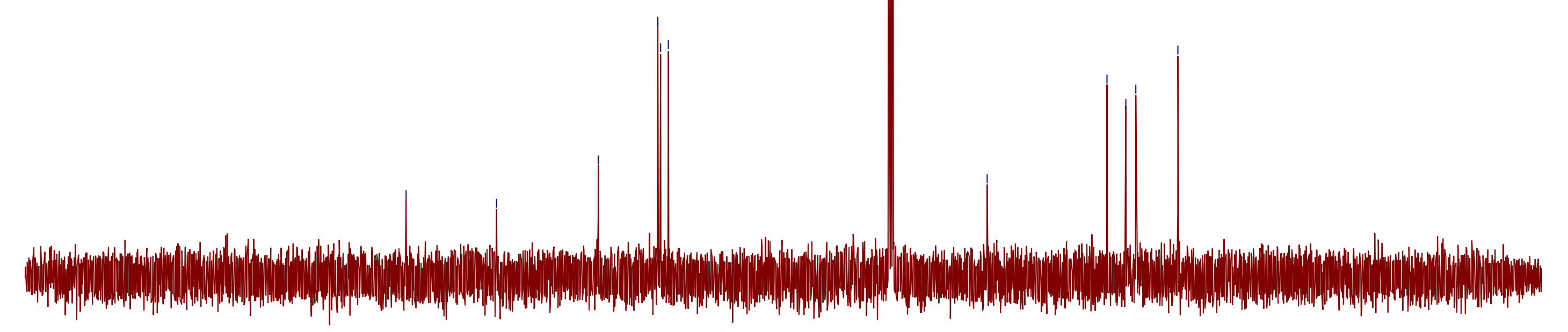

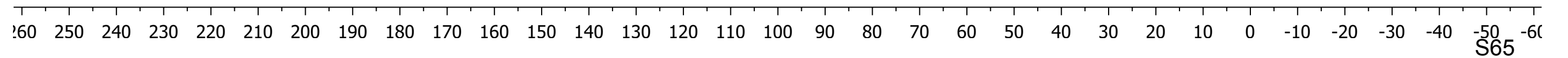




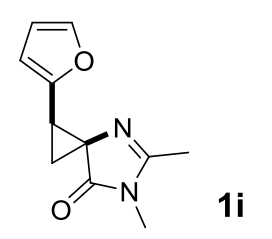

${ }^{1} \mathrm{H}$ NMR (300 MHz), $\mathrm{CDCl}_{3}$

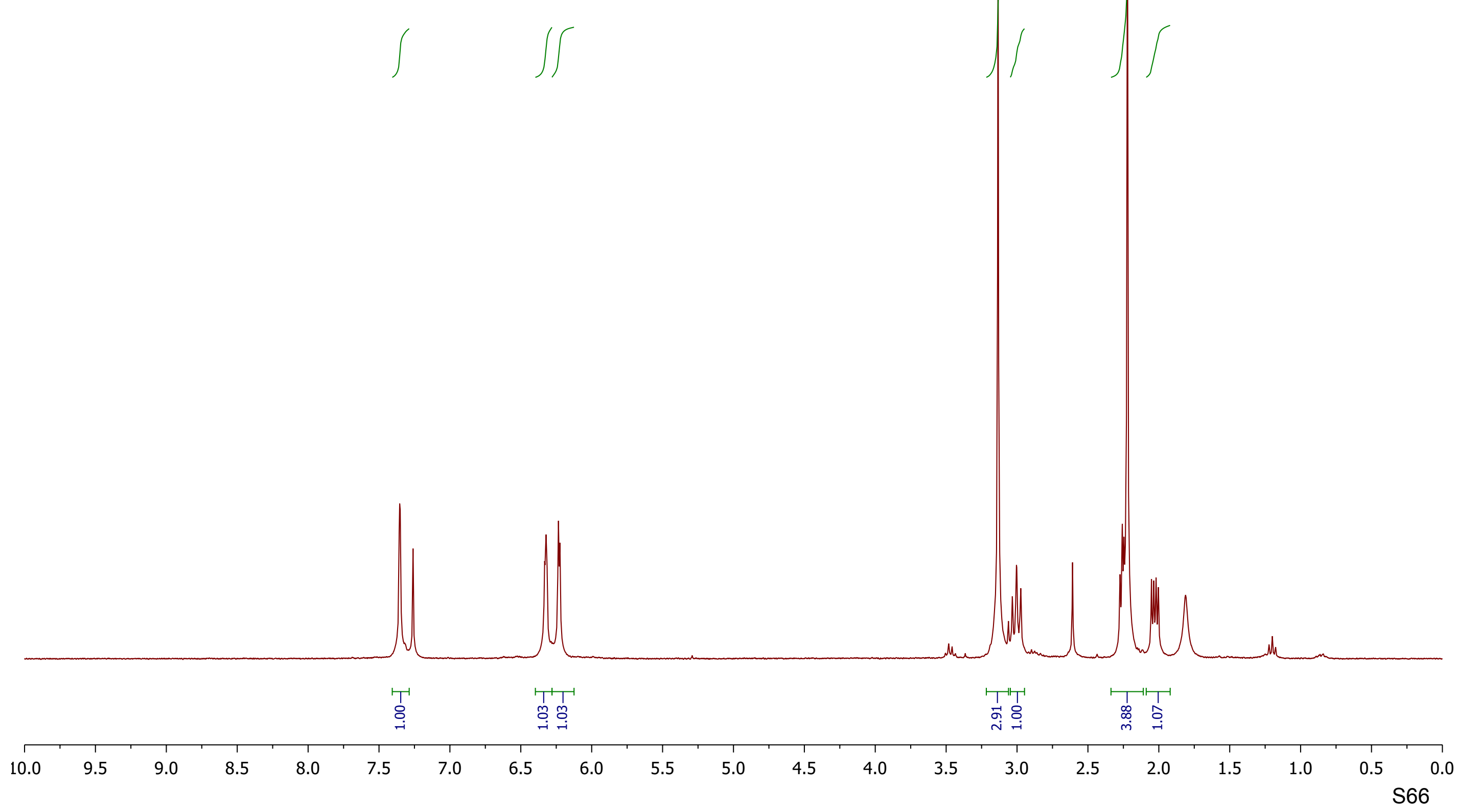




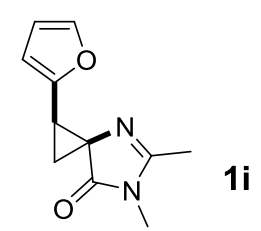

${ }^{13} \mathrm{C} \mathrm{NMR}$ (75 MHz), $\mathrm{CDCl}_{3}$

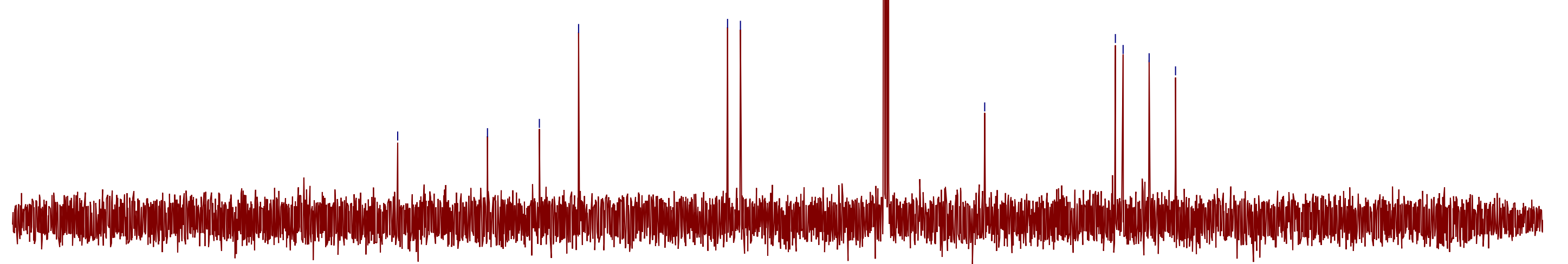

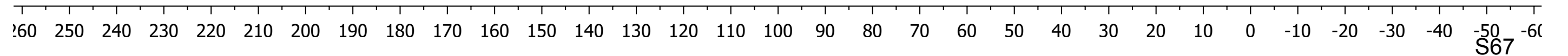



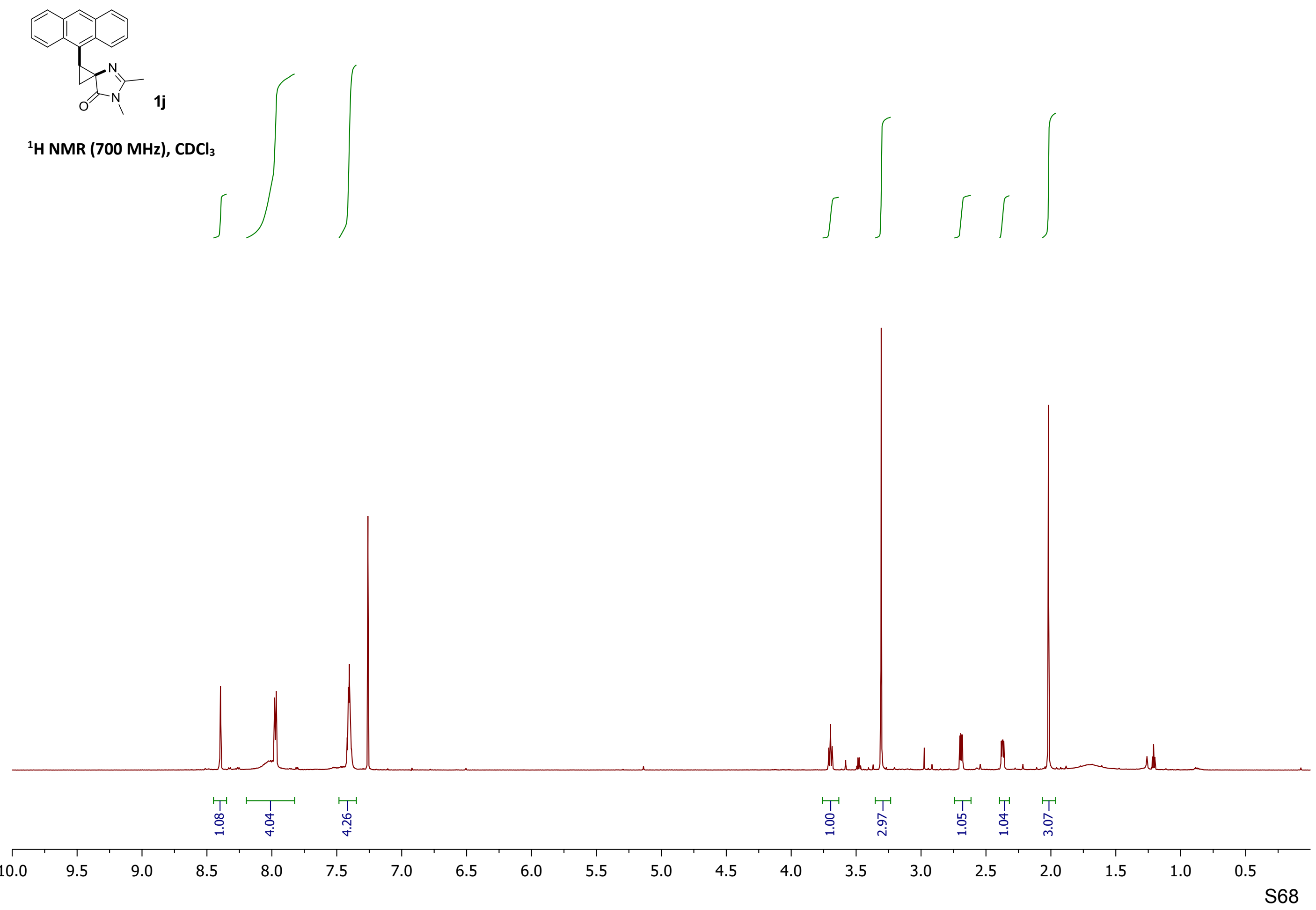


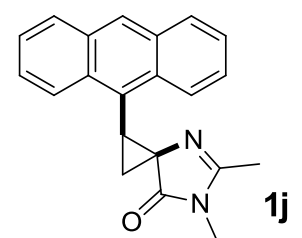

${ }^{13} \mathrm{C} \mathrm{NMR} \mathrm{(75} \mathrm{MHz),} \mathrm{CDCl}_{3}$

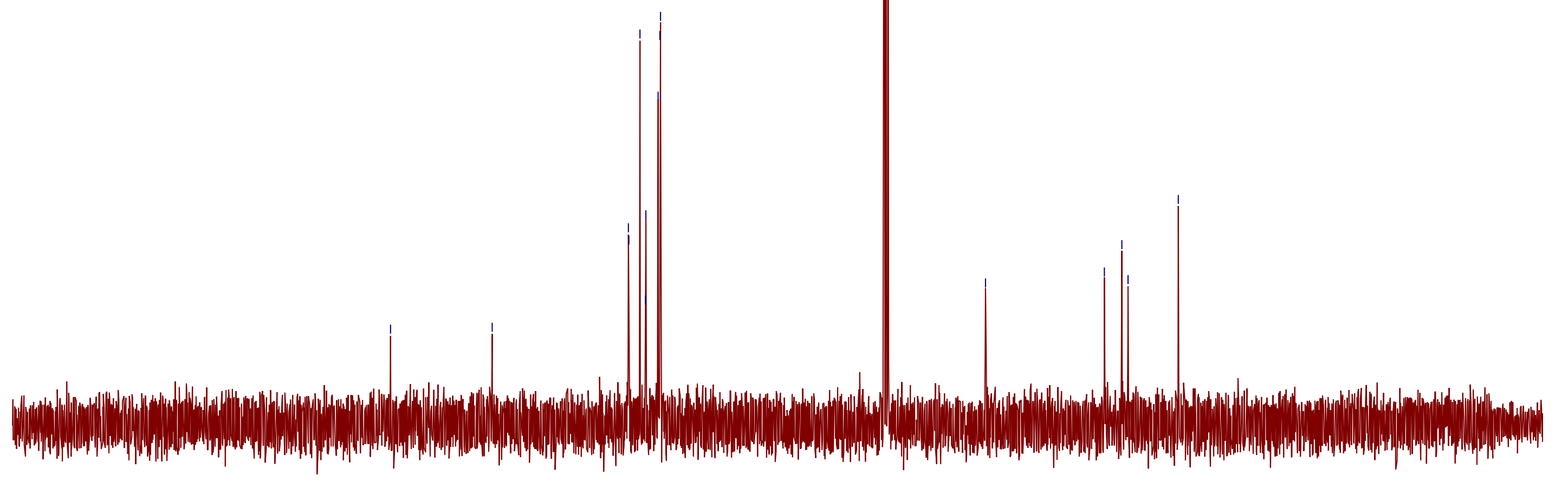

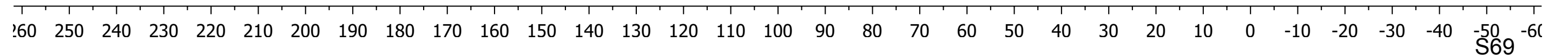




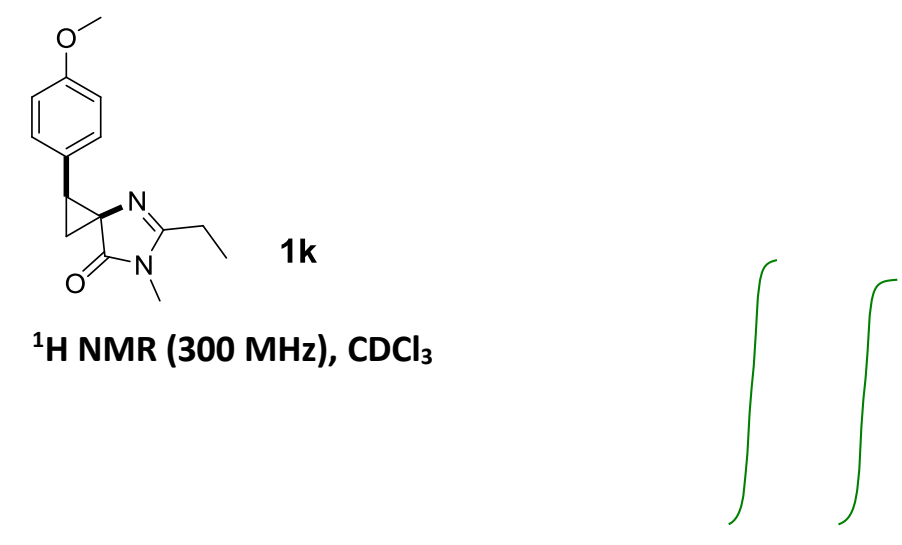


${ }^{1} \mathrm{H}$ NMR (300 MHz), $\mathrm{CDCl}_{3}$
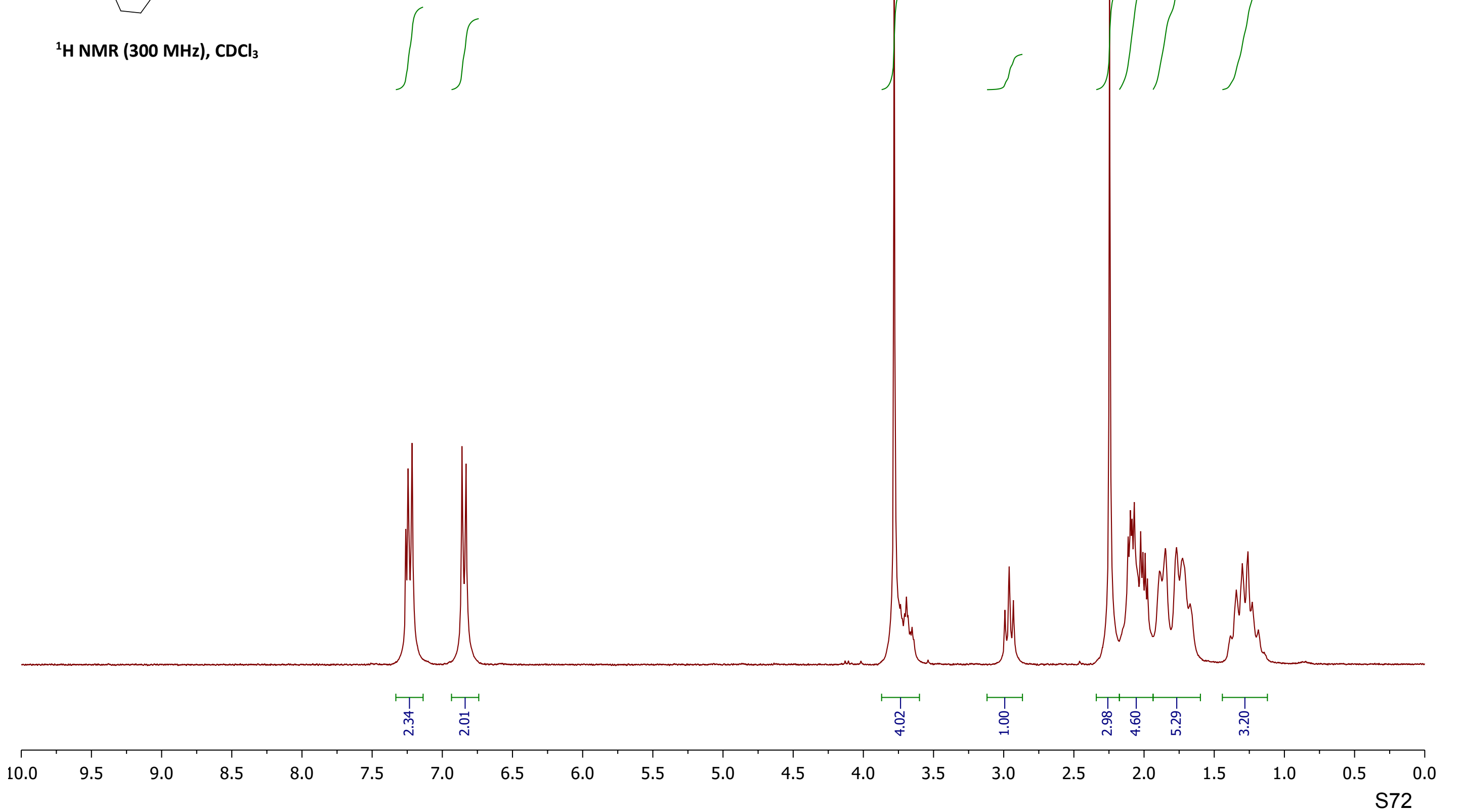
${ }^{13} \mathrm{C} \mathrm{NMR}(75 \mathrm{MHz}), \mathrm{CDCl}_{3}$

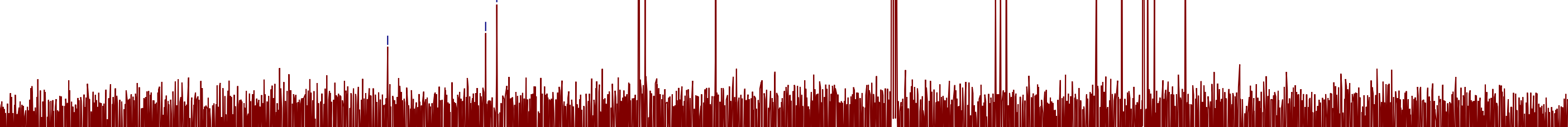

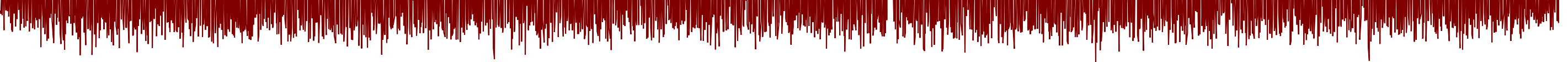

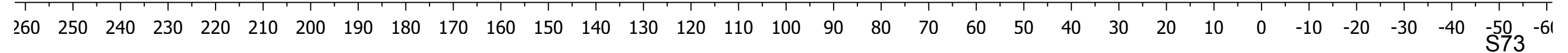




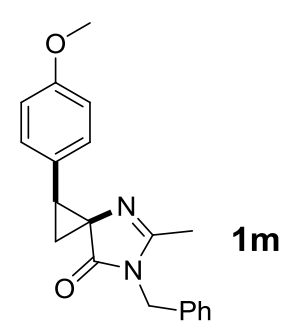

${ }^{1} \mathrm{H}$ NMR (300 MHz), $\mathrm{CDCl}_{3}$
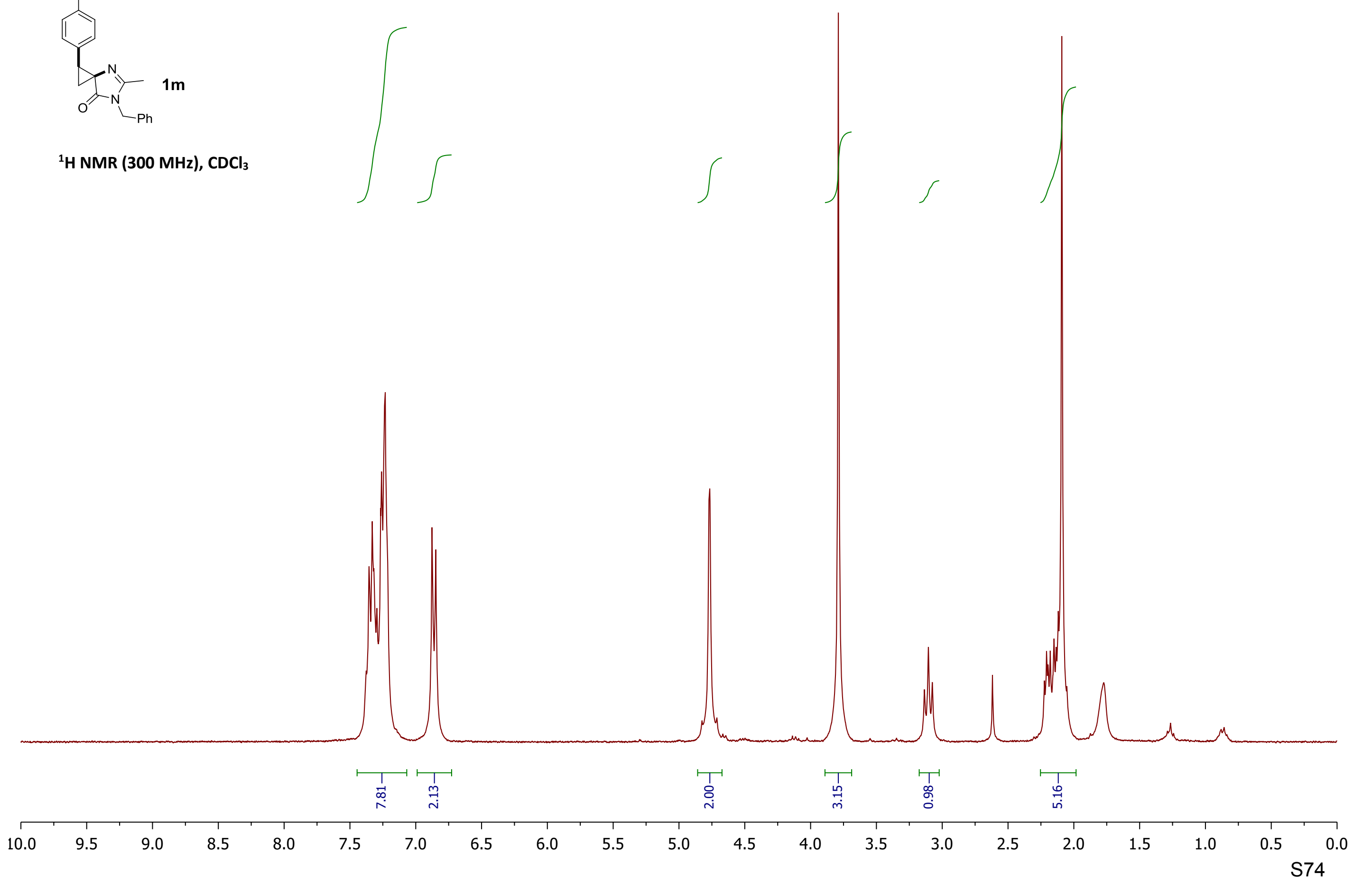


\section{${ }^{13} \mathrm{C} \mathrm{NMR}(75 \mathrm{MHz}), \mathrm{CDCl}_{3}$}

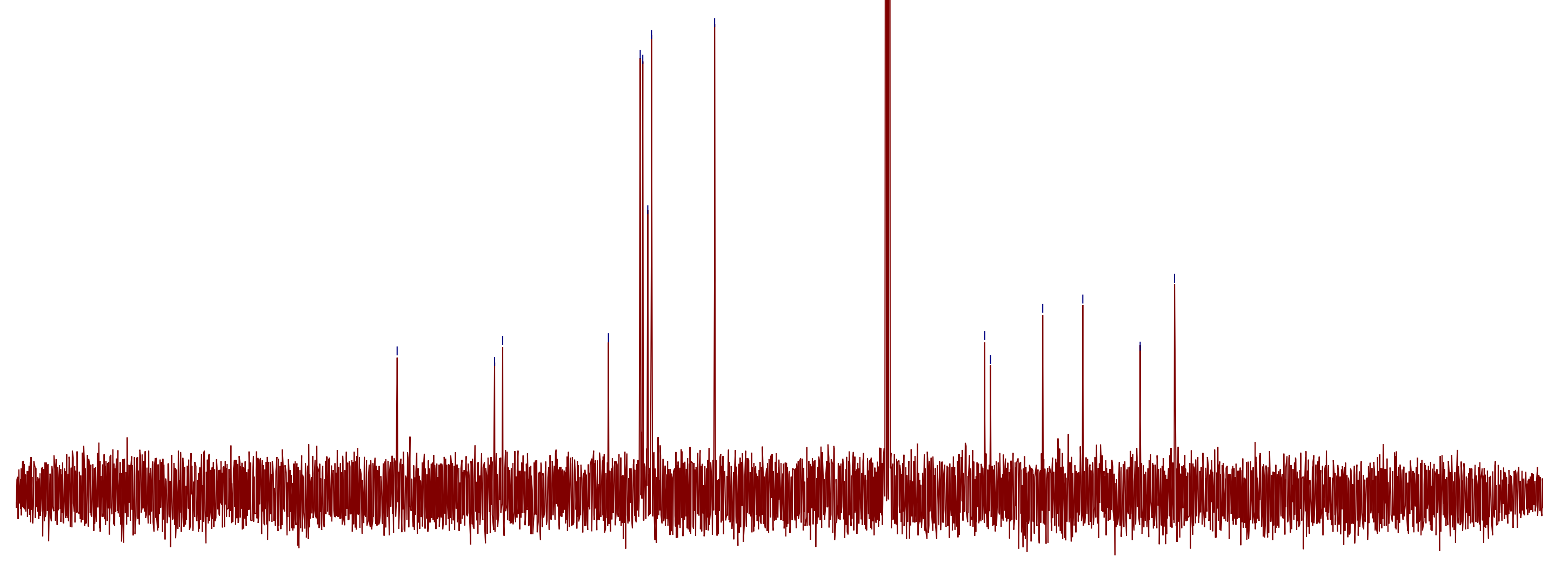

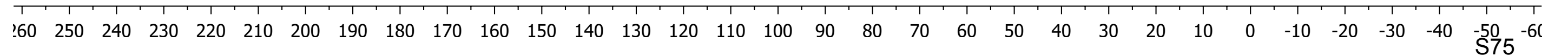




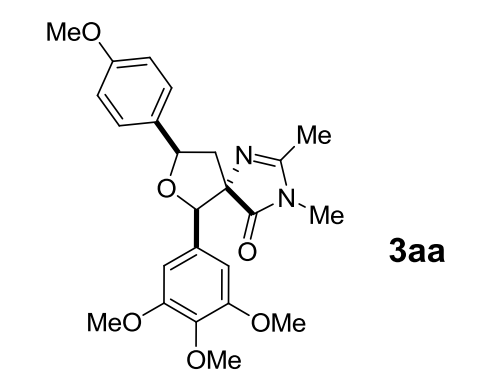

${ }^{1} \mathrm{H} \mathrm{NMR}(700 \mathrm{MHz}), \mathrm{CDCl}_{3}$

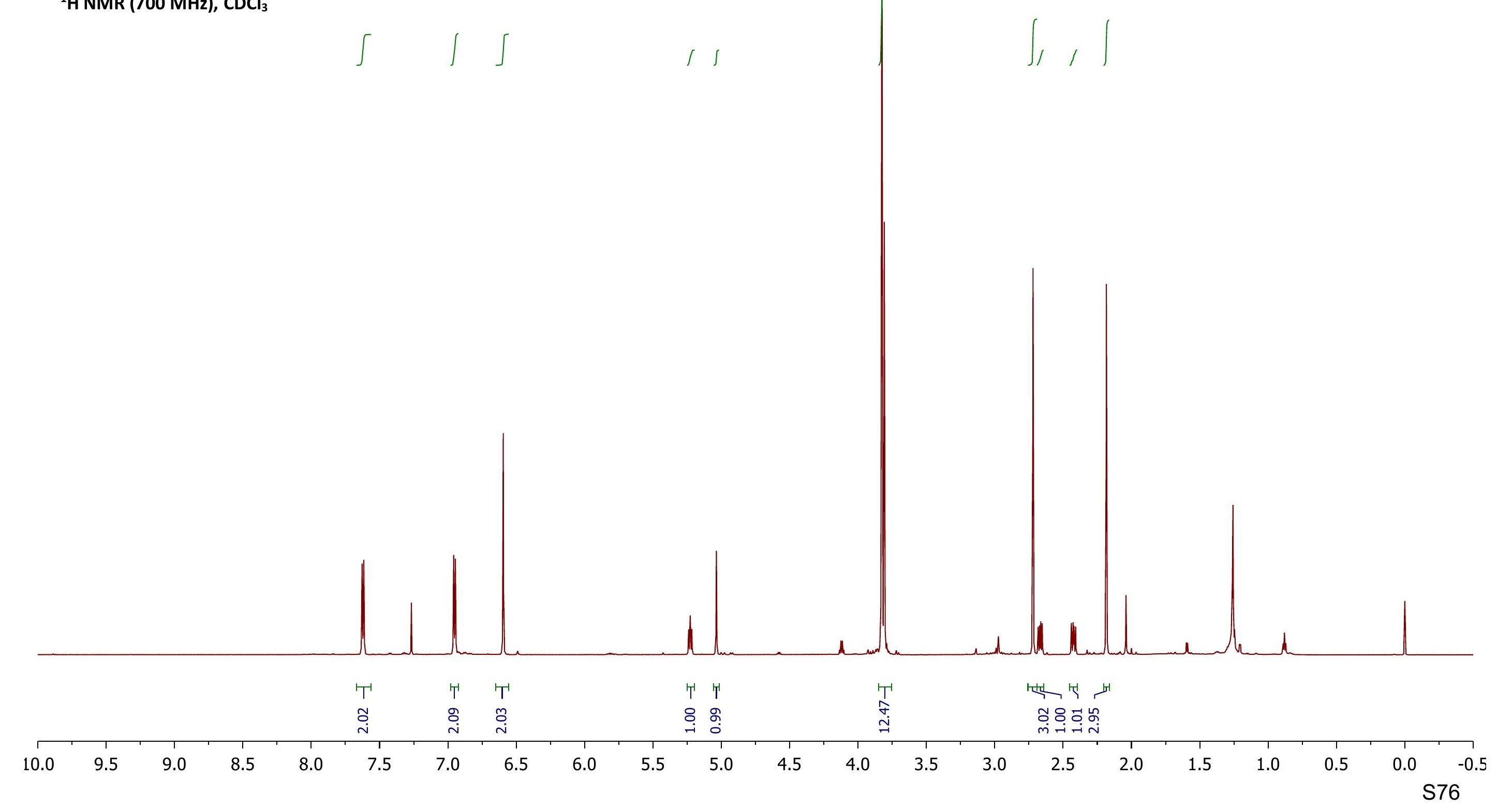


$\mathrm{MeO}$

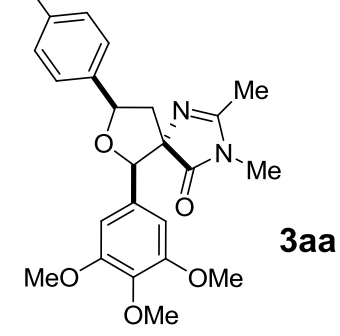

${ }^{13} \mathrm{C}$ NMR (175 MHz), $\mathrm{CDCl}_{3}$

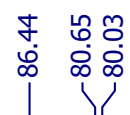

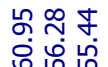

ชั่ นึ่

$\stackrel{\stackrel{n}{m}}{\stackrel{f}{*}}$

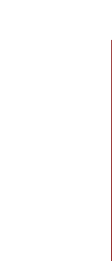

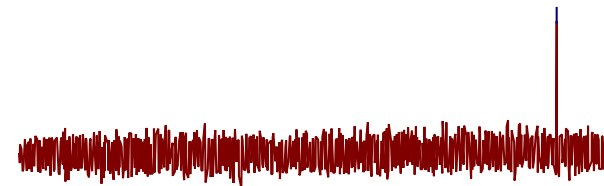




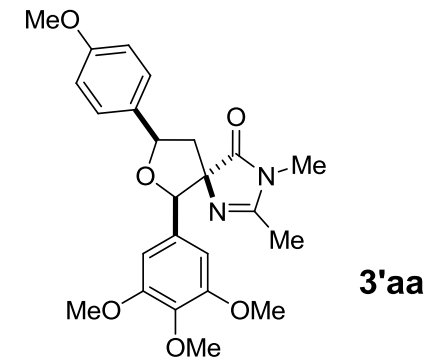

${ }^{1} \mathrm{H}$ NMR (700 MHz), $\mathrm{CDCl}_{3}$

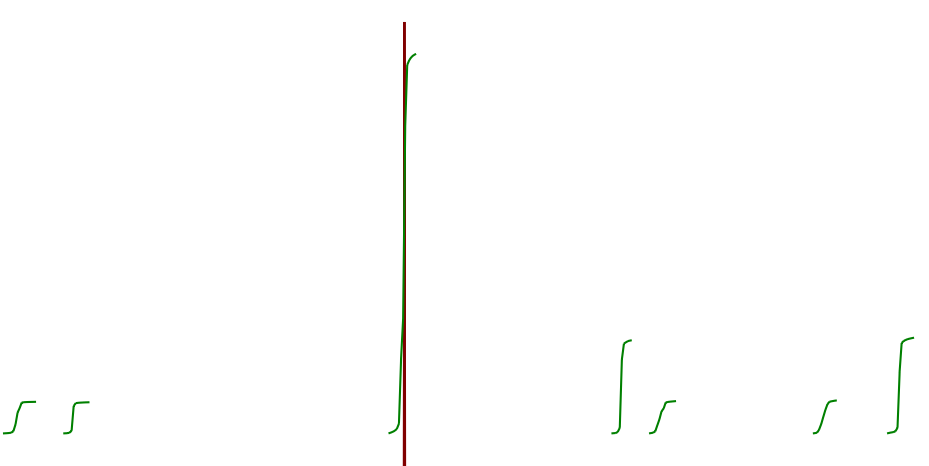




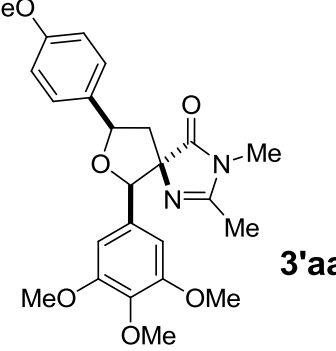

${ }^{1} \mathrm{H}$ NMR (300 MHz), DMSO-d

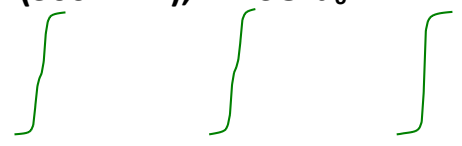

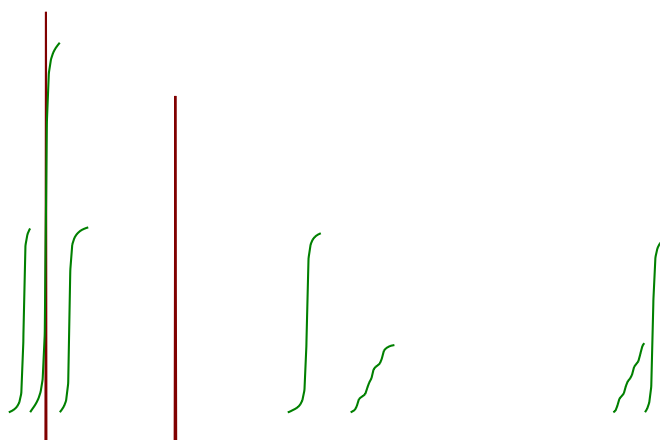

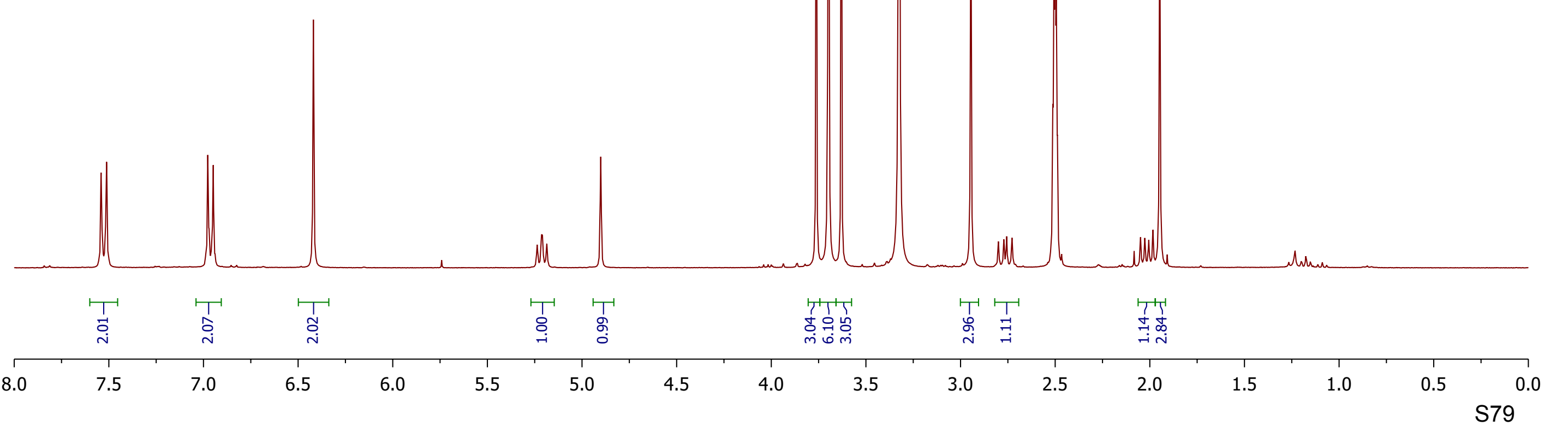




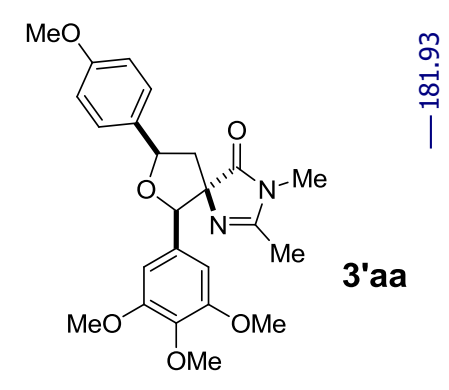

${ }^{13} \mathrm{C}$ NMR (75 MHz), DMSO-d 6

\begin{tabular}{|c|c|c|c|c|c|c|c|c|c|c|c|c|c|c|c|c|c|c|c|c|}
\hline 20 & 210 & 200 & 190 & 180 & 170 & 160 & 150 & 140 & 130 & 120 & 110 & 100 & 90 & 80 & 70 & 60 & 50 & 40 & 30 & ${ }^{10} \mathrm{~S} 80$ \\
\hline
\end{tabular}




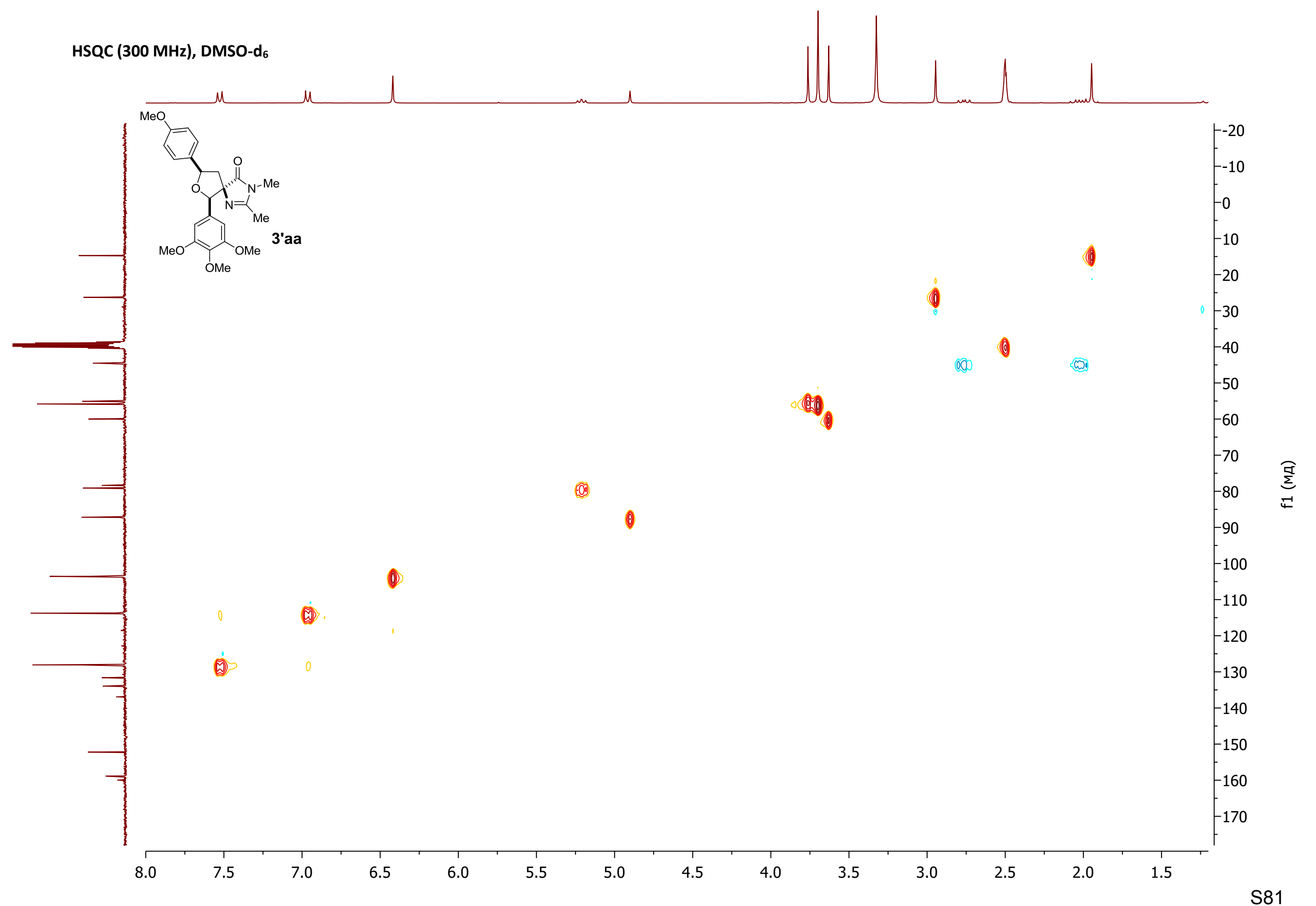




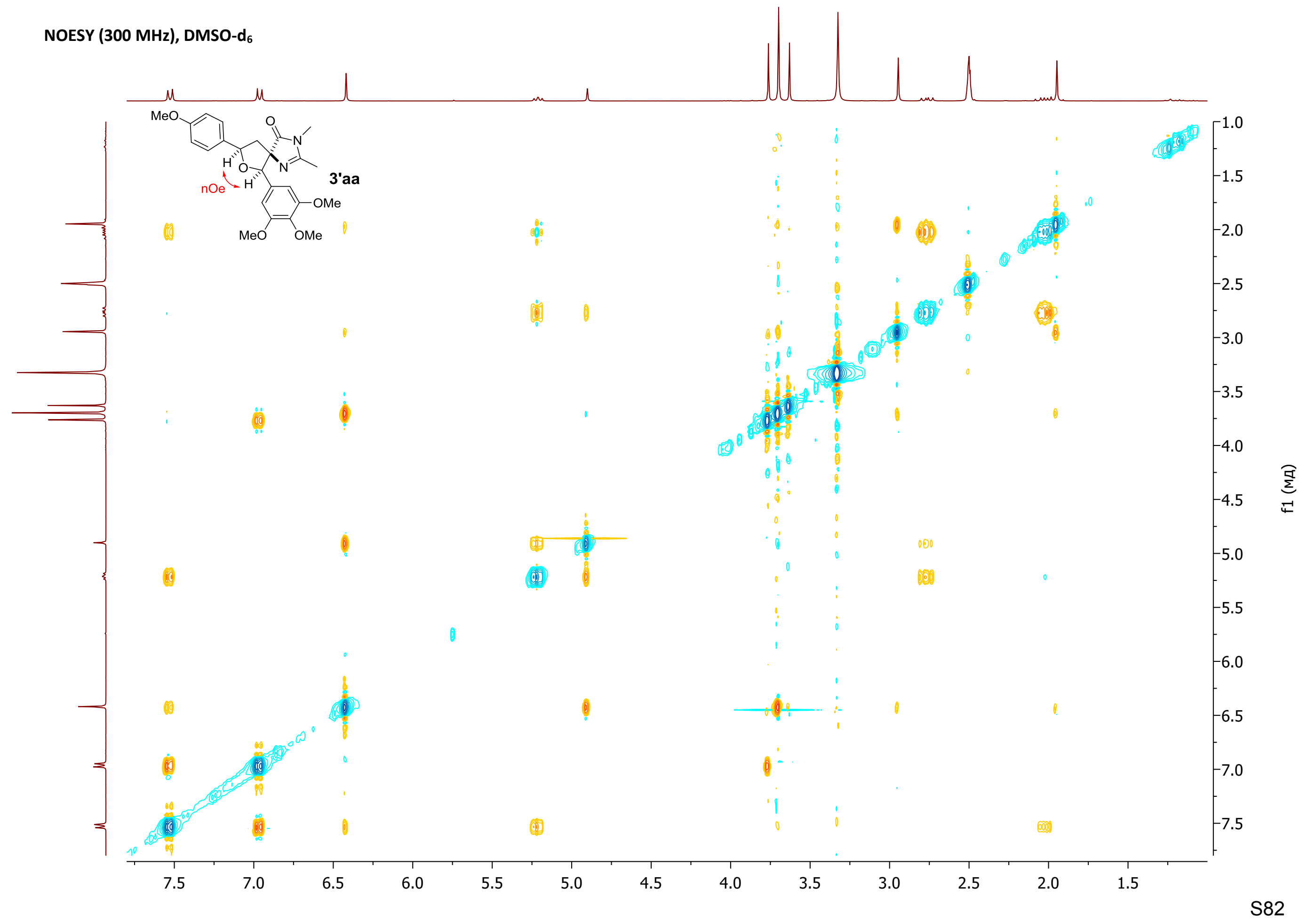


$\mathrm{MeO}$

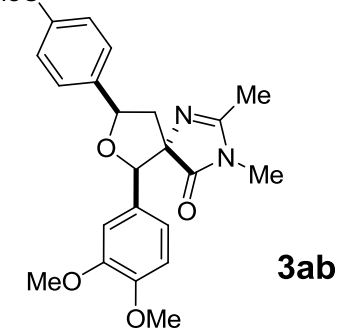

${ }^{1} \mathrm{H}$ NMR (700 MHz), $\mathrm{CDCl}_{3}$

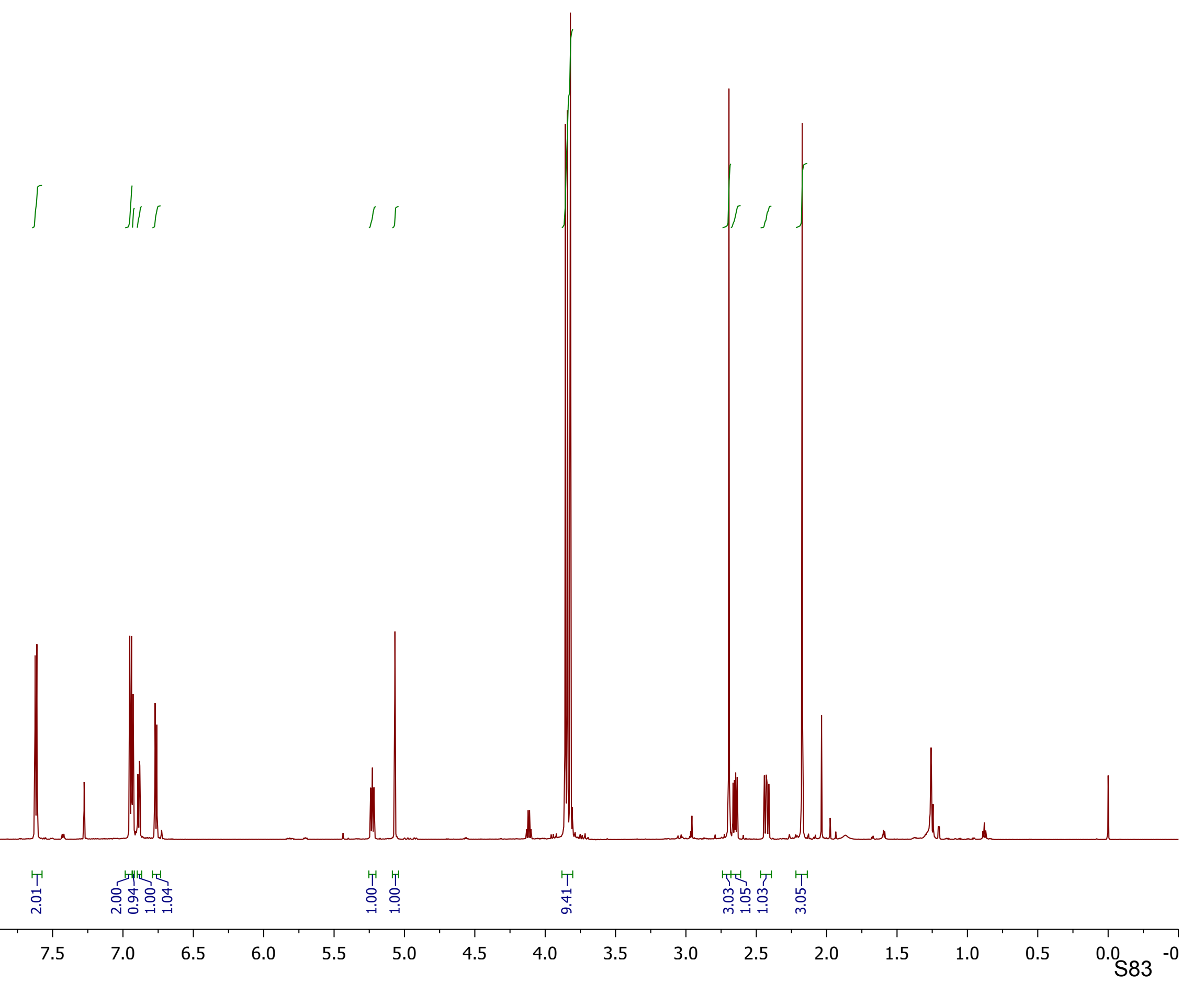


$\mathrm{MeO}$

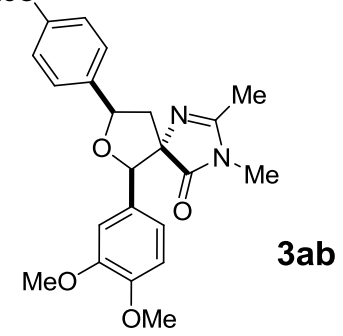

${ }^{13} \mathrm{C}$ NMR (175 MHz), $\mathrm{CDCl}_{3}$

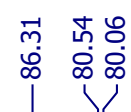

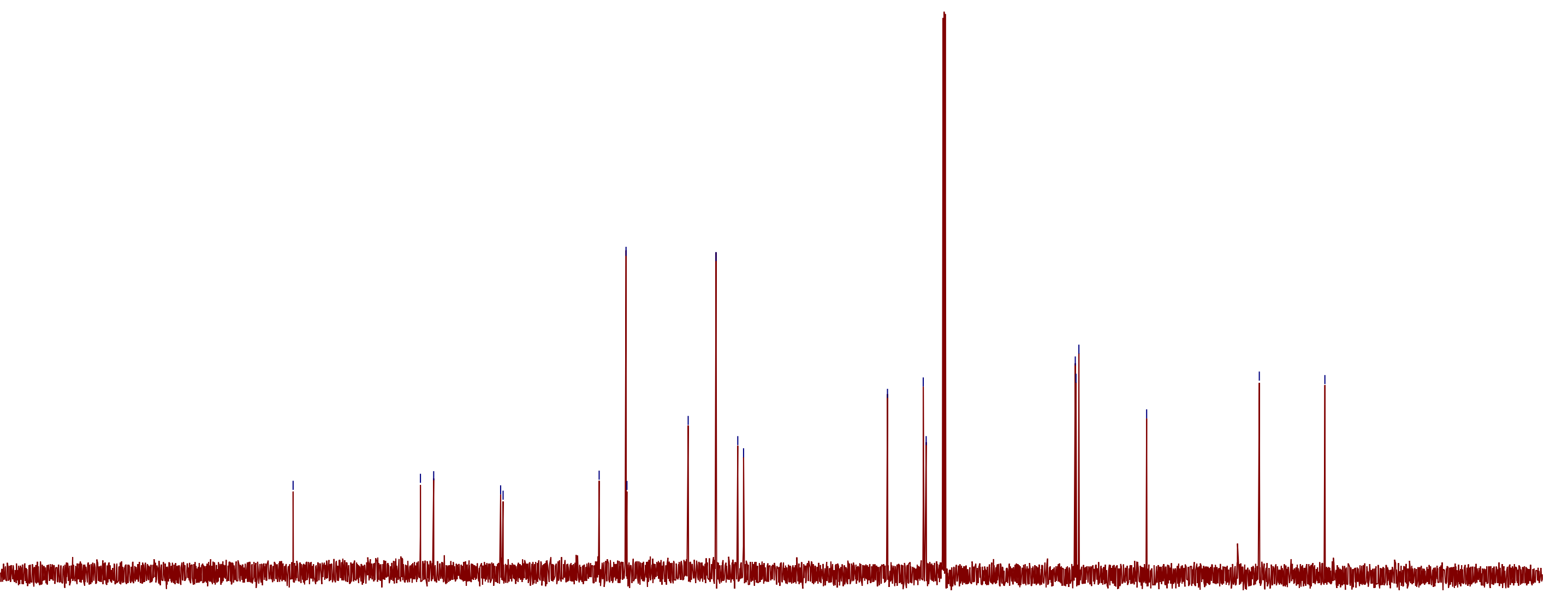


$\mathrm{MeO}$

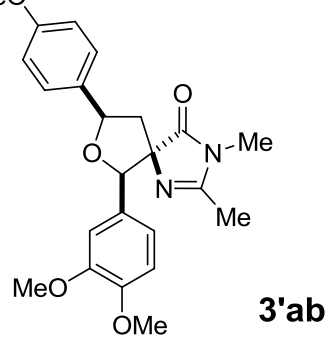

${ }^{1} \mathrm{H}$ NMR (700 MHz), $\mathrm{CDCl}_{3}$
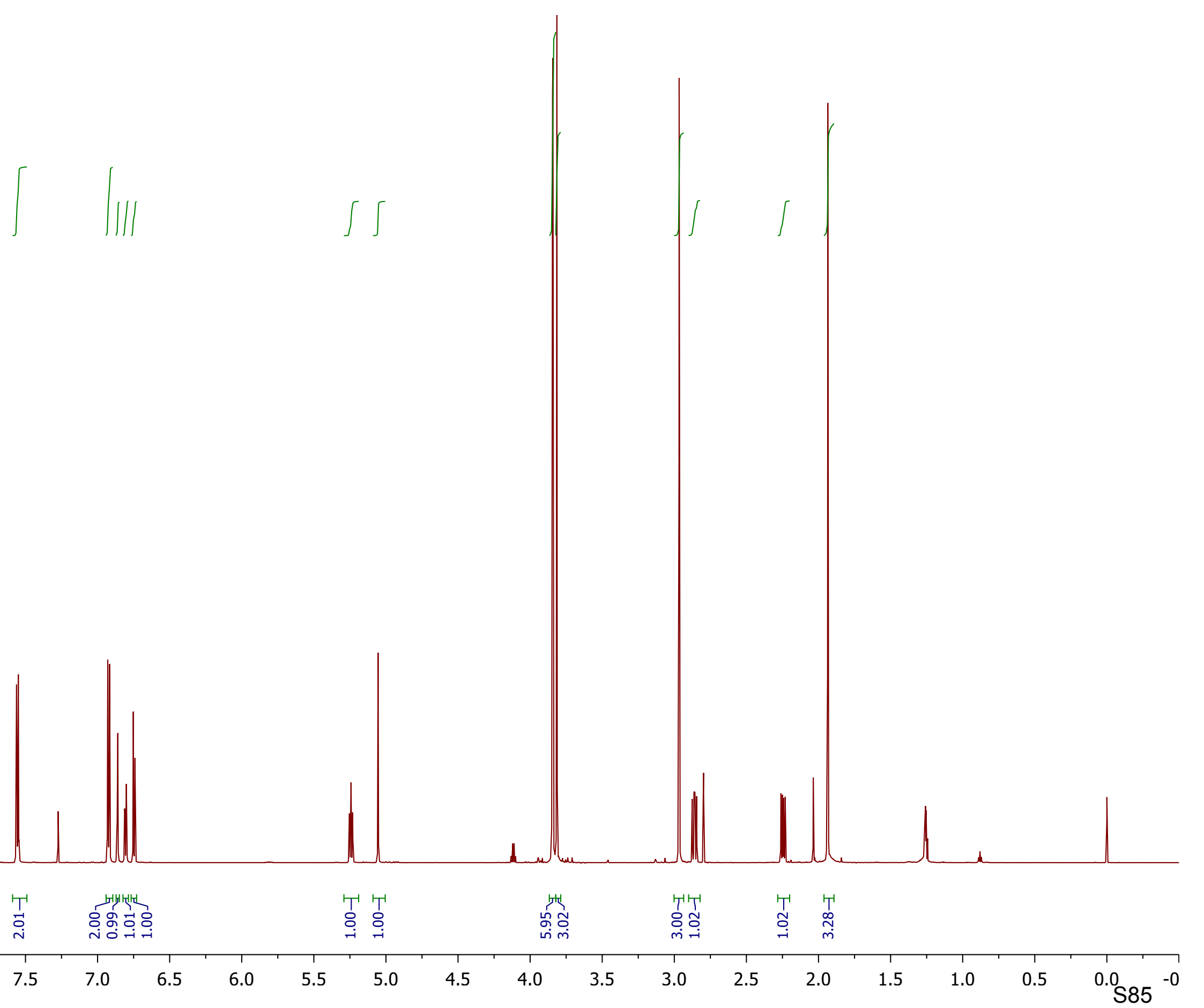


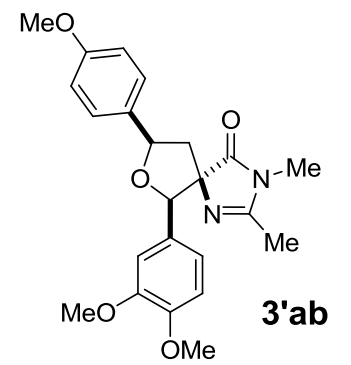

${ }^{13} \mathrm{C} \mathrm{NMR}(75 \mathrm{MHz}), \mathrm{CDCl}_{3}$

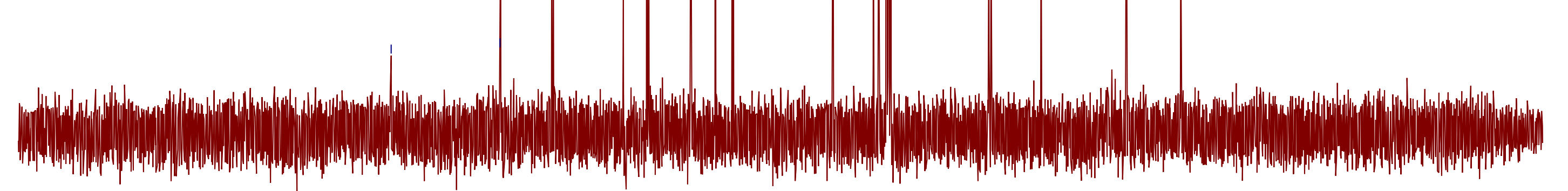

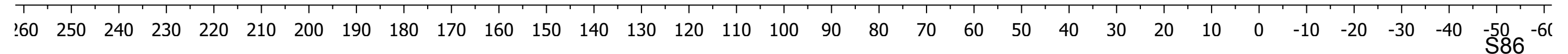




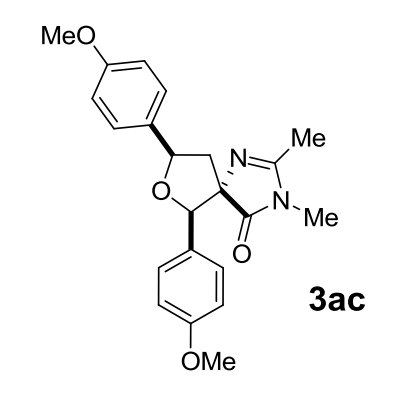

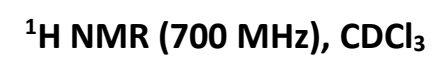
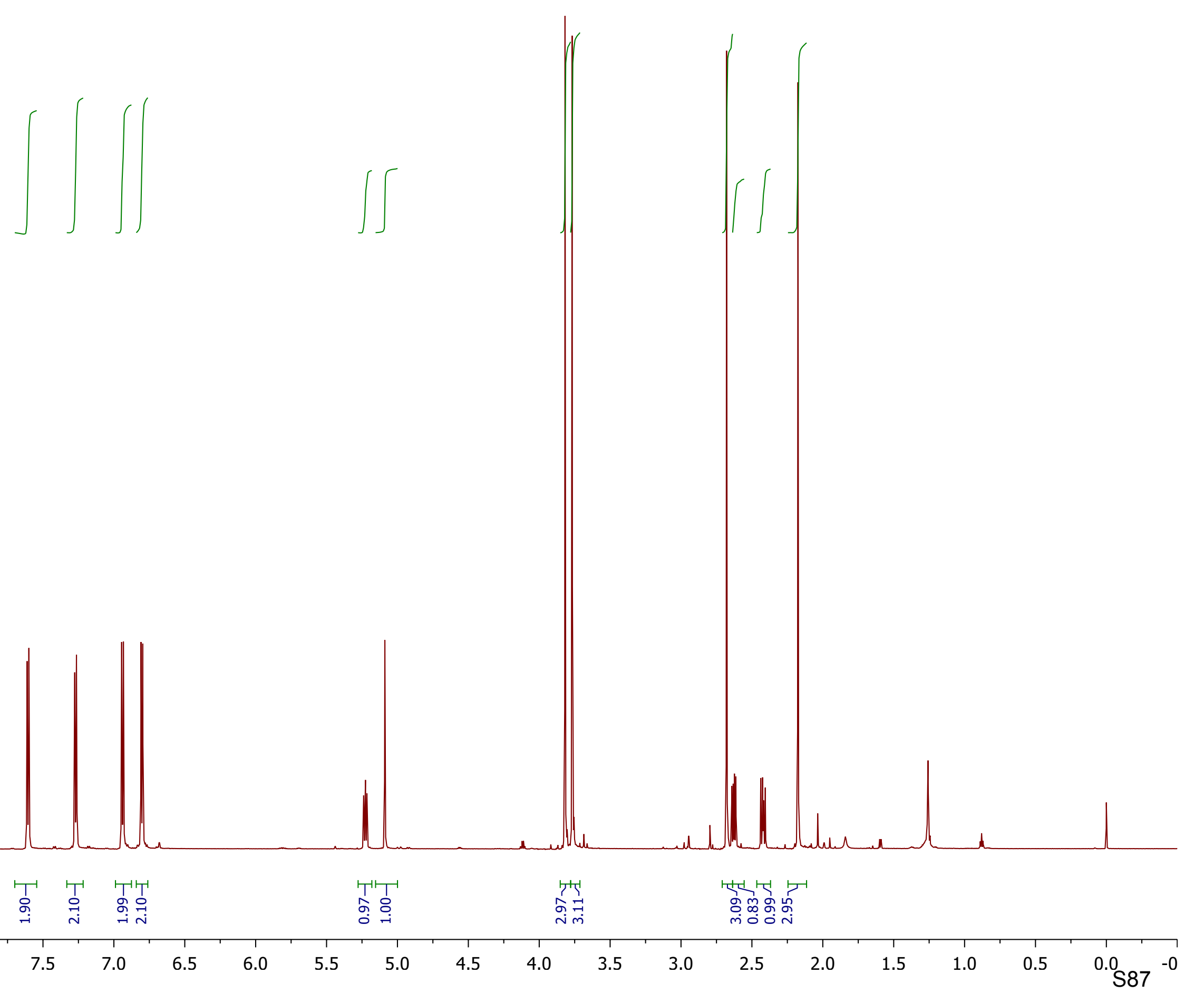


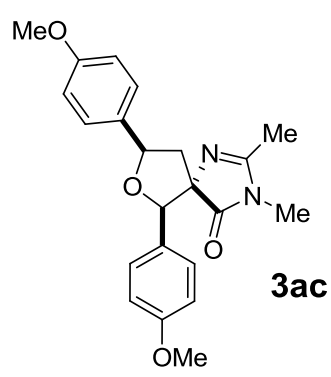

${ }^{13} \mathrm{C} \mathrm{NMR} \mathrm{(175} \mathrm{MHz),} \mathrm{CDCl}_{3}$

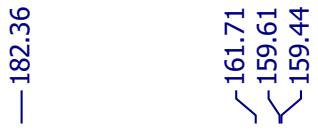

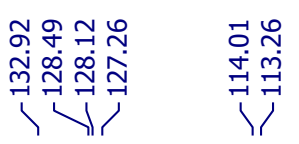

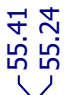

$\stackrel{\vec{b}}{\dot{f}}$
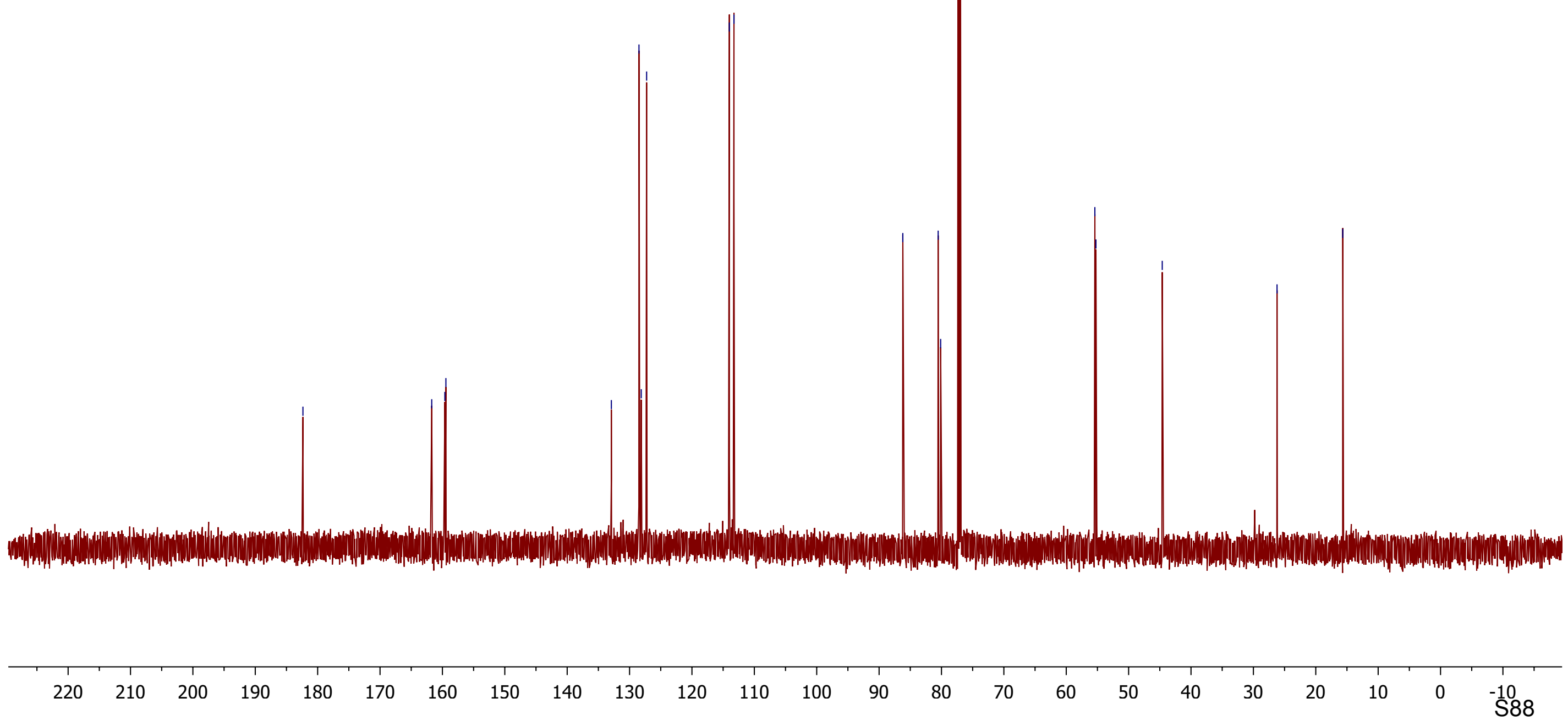


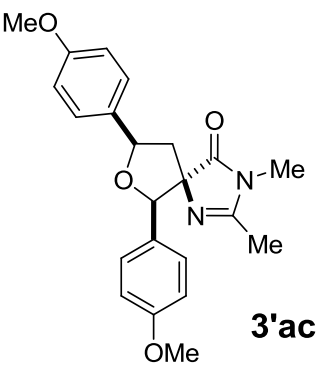

${ }^{1} \mathrm{H}$ NMR (700 MHz), $\mathrm{CDCl}_{3}$
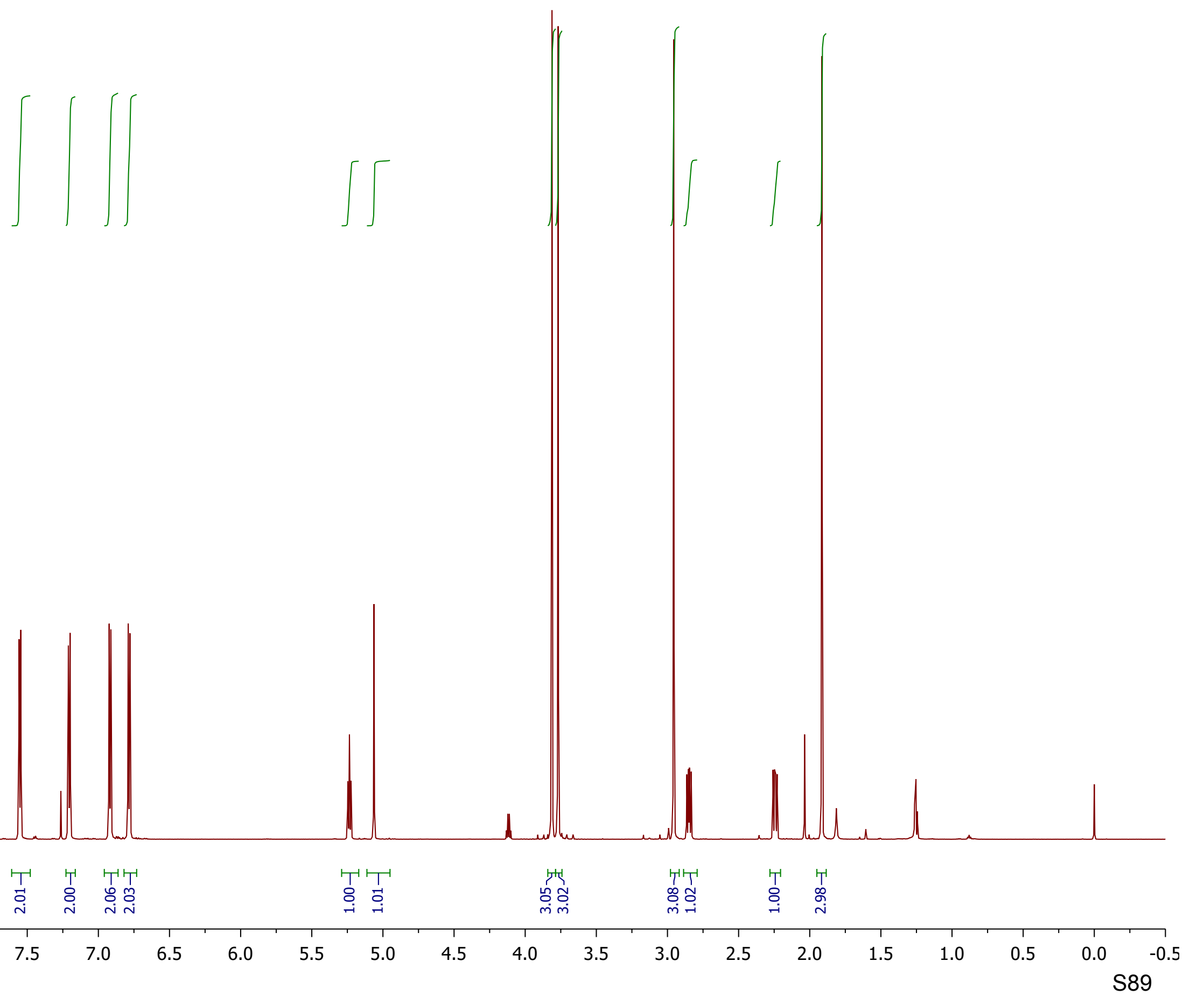
$\mathrm{MeO}$

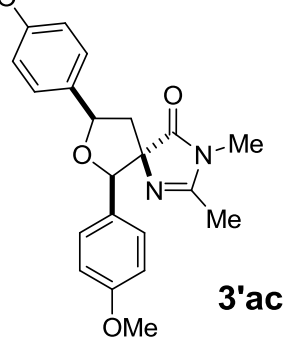

${ }^{13} \mathrm{C} \mathrm{NMR} \mathrm{(175} \mathrm{MHz),} \mathrm{CDCl}_{3}$

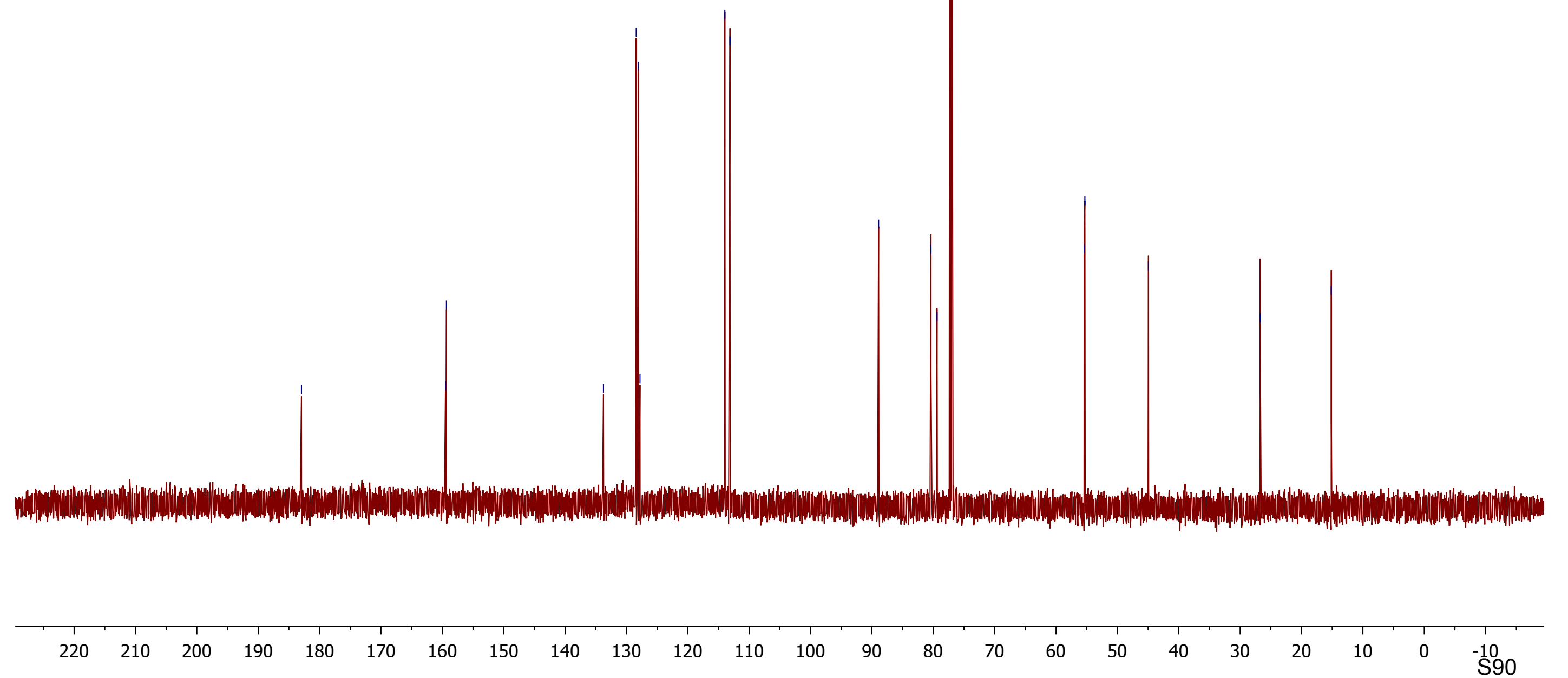


$\mathrm{MeO}$

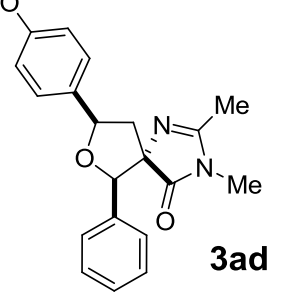

${ }^{1} \mathrm{H}$ NMR (700 MHz), $\mathrm{CDCl}_{3}$
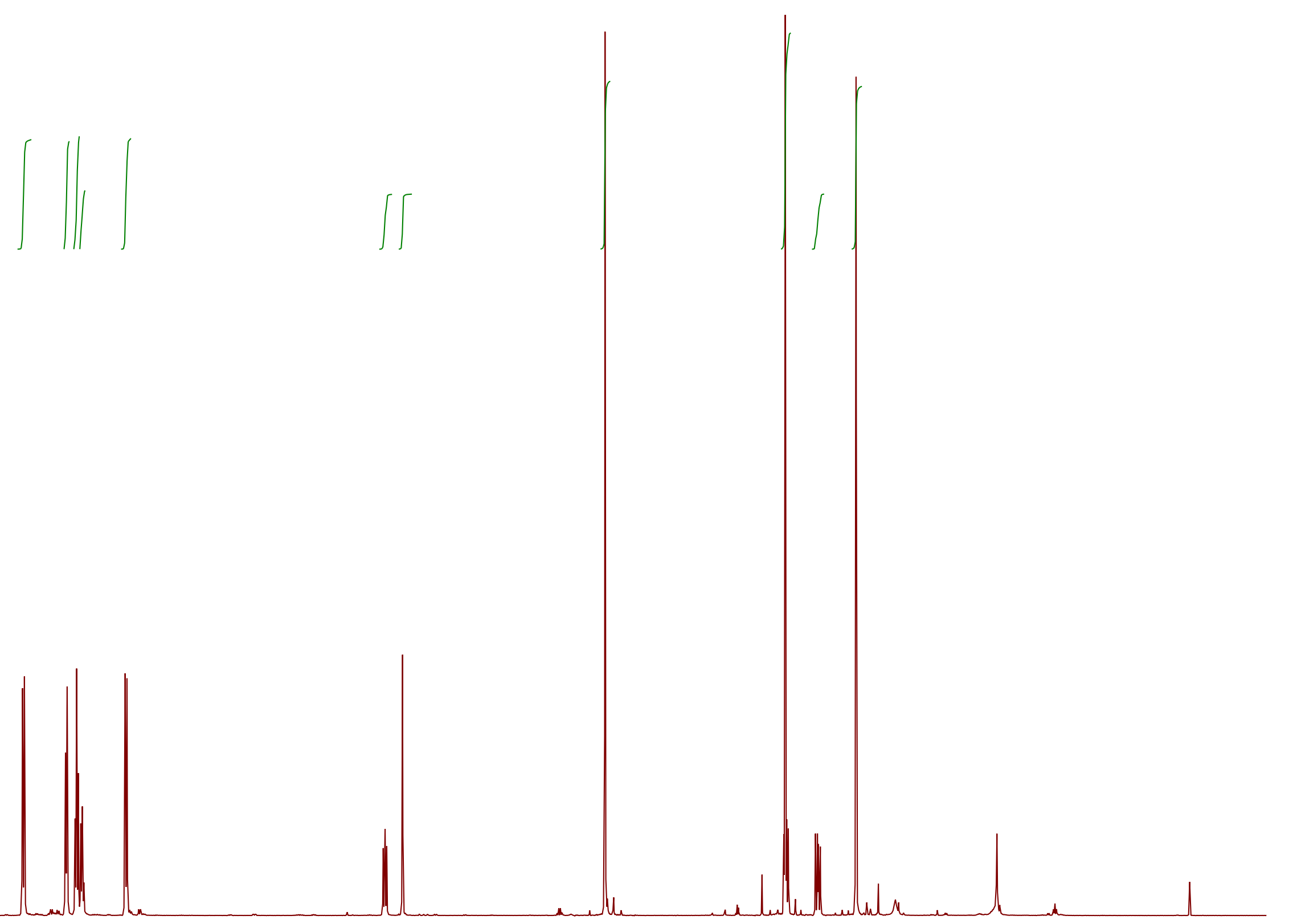

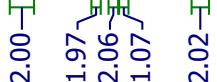

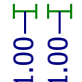

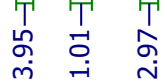




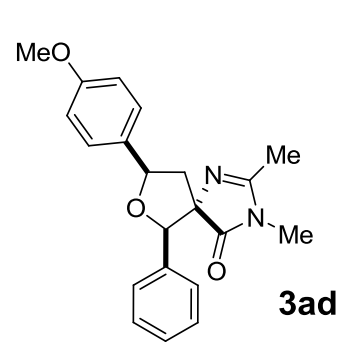

年

|

$\mid$

㿠

i.

${ }^{13} \mathrm{C}$ NMR (175 MHz), CDCl 3
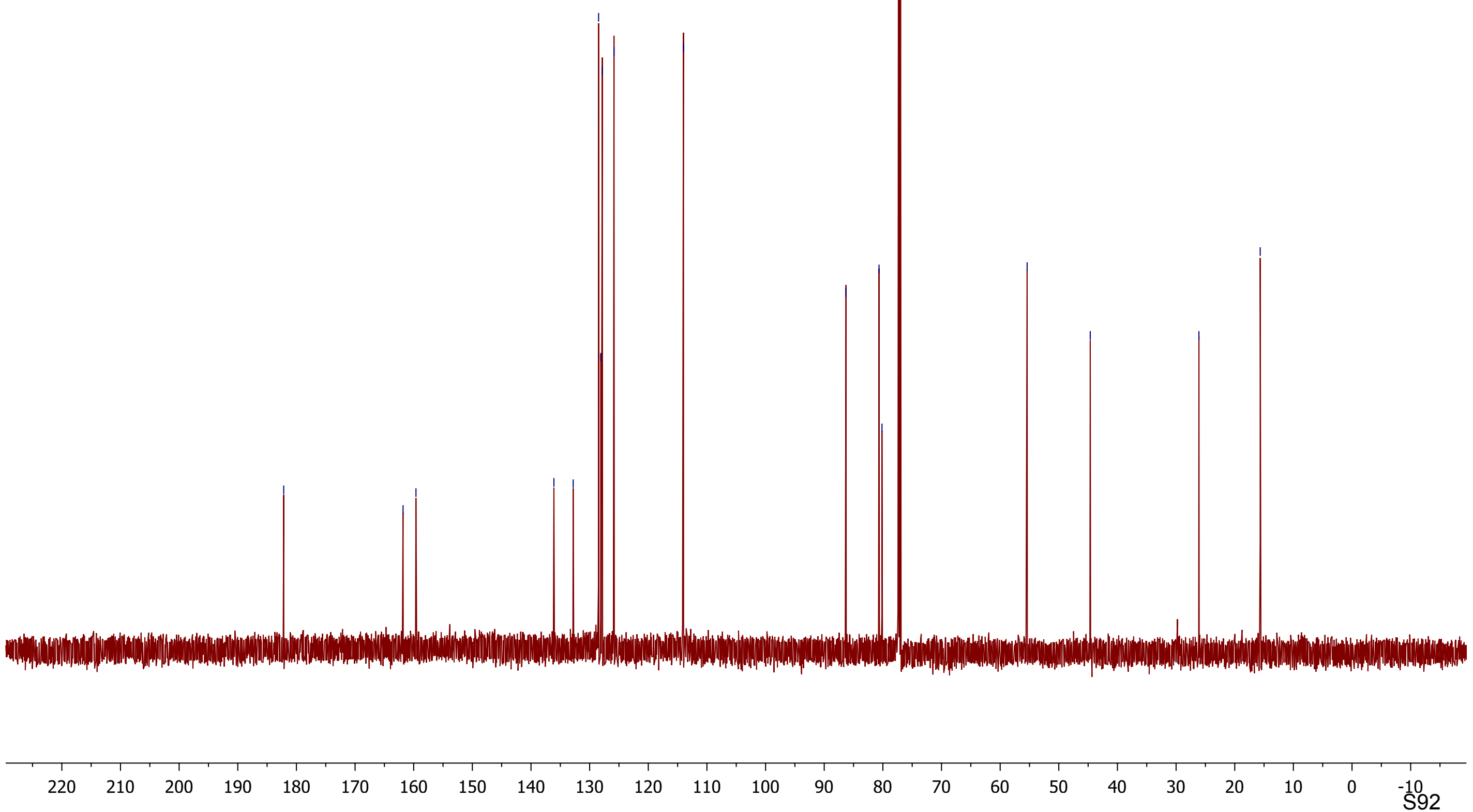
$\mathrm{MeO}$

(3)

${ }^{1} \mathrm{H}$ NMR (700 MHz), $\mathrm{CDCl}_{3}$
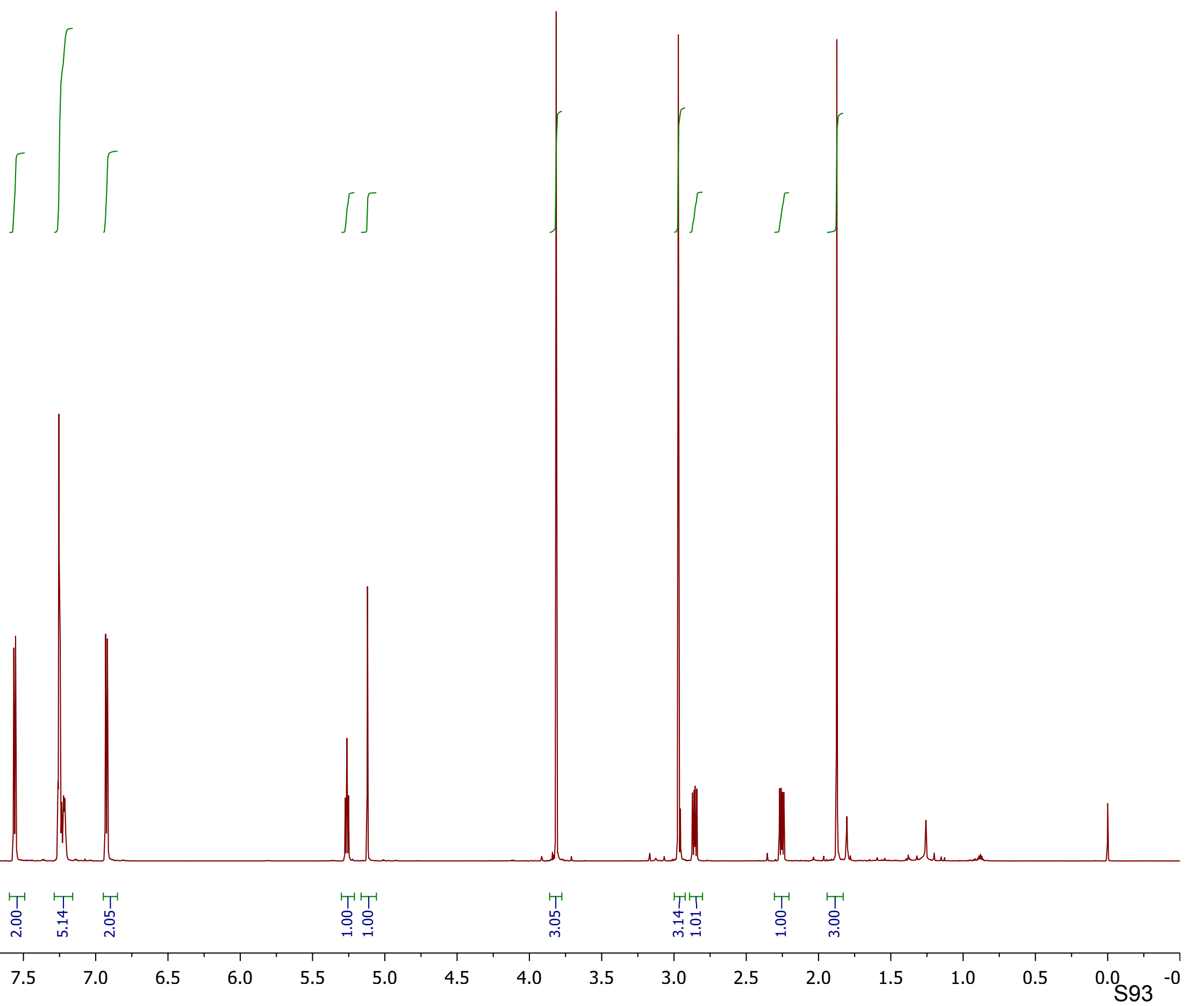


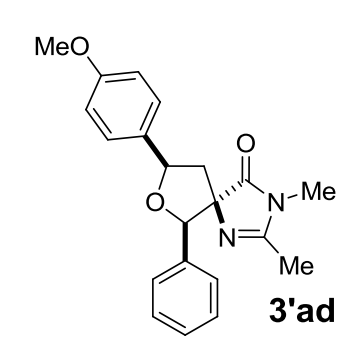

${ }^{13} \mathrm{C} \mathrm{NMR} \mathrm{(75} \mathrm{MHz),} \mathrm{CDCl}_{3}$
|

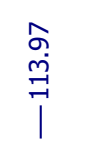

$\stackrel{\infty}{\stackrel{\infty}{i}}$

$\stackrel{\infty}{\stackrel{\infty}{+}}$
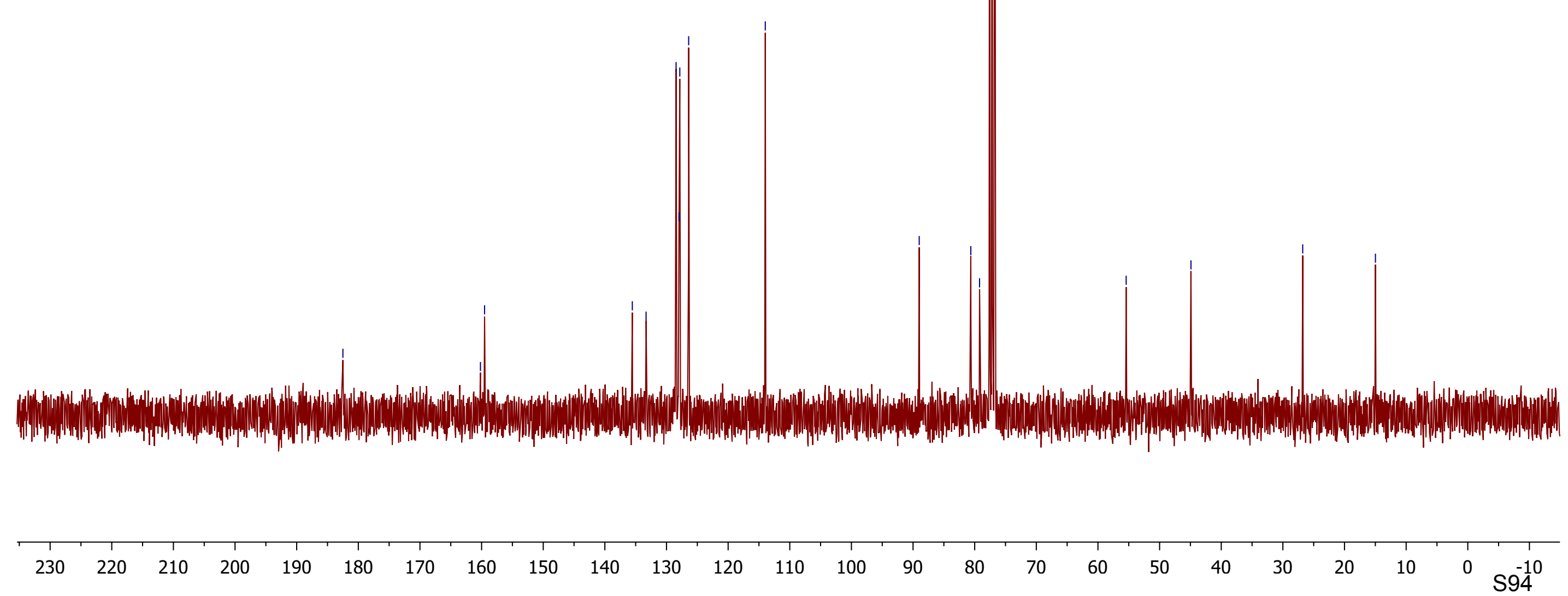
$\mathrm{MeO}$

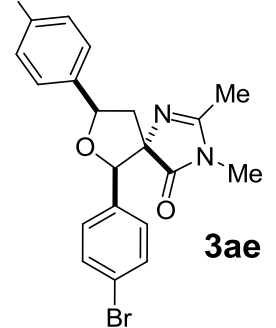

${ }^{1} \mathrm{H}$ NMR (700 MHz), $\mathrm{CDCl}_{3}$
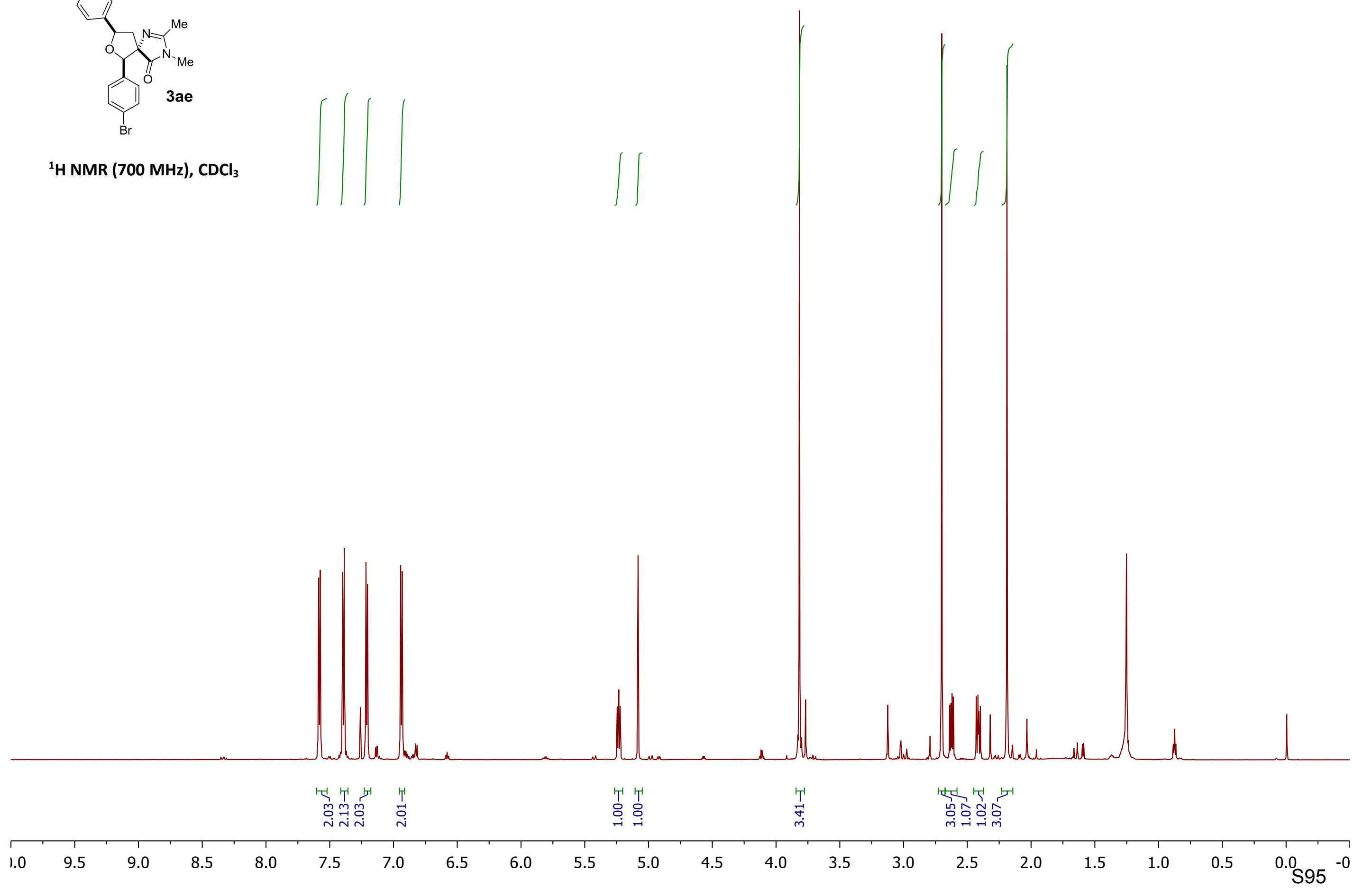
$\mathrm{MeO}$

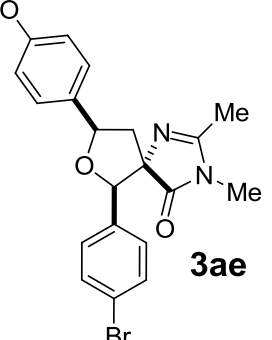

${ }^{13} \mathrm{C}$ NMR (175 MHz), $\mathrm{CDCl}_{3}$
ชัฒ

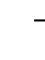



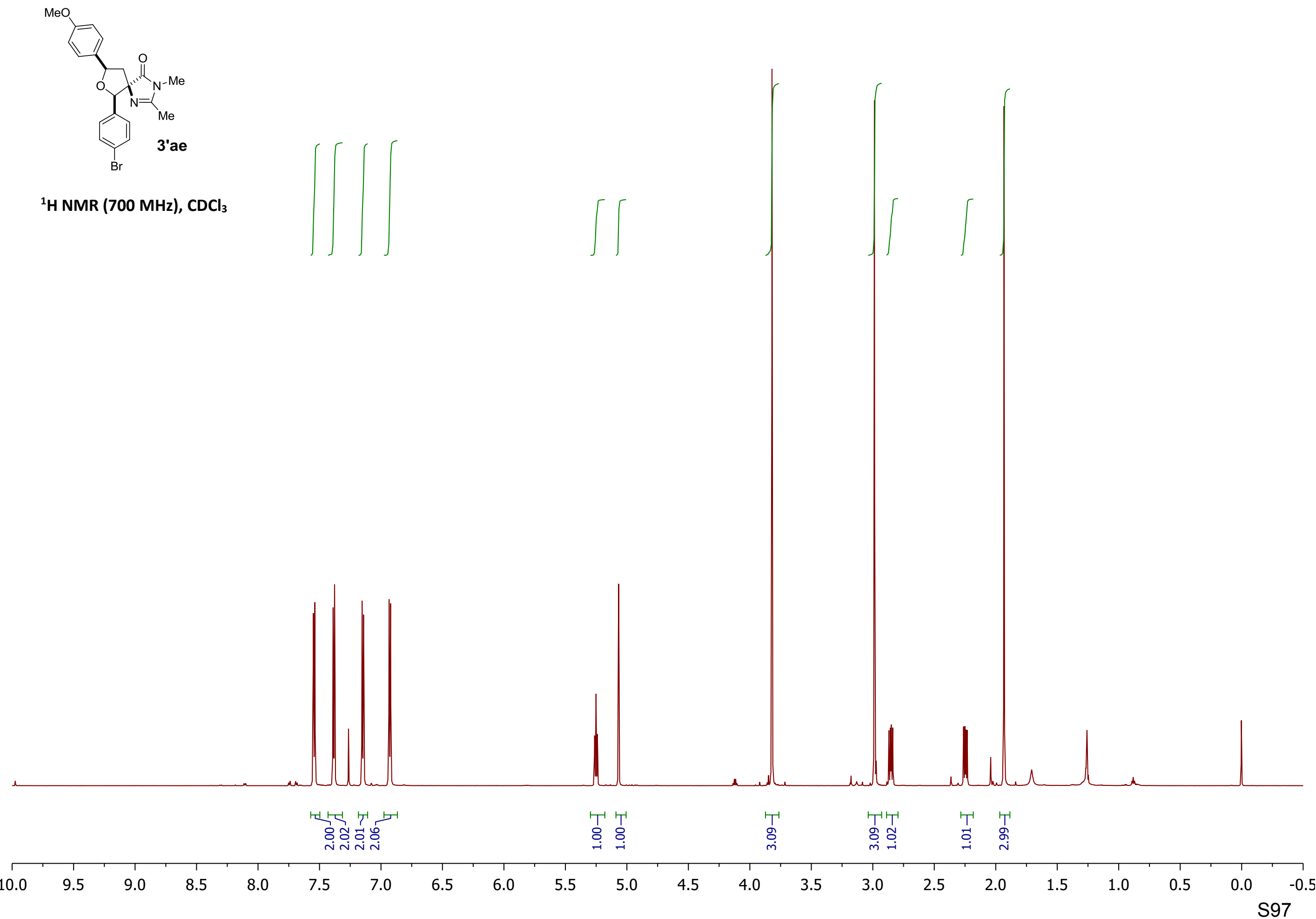

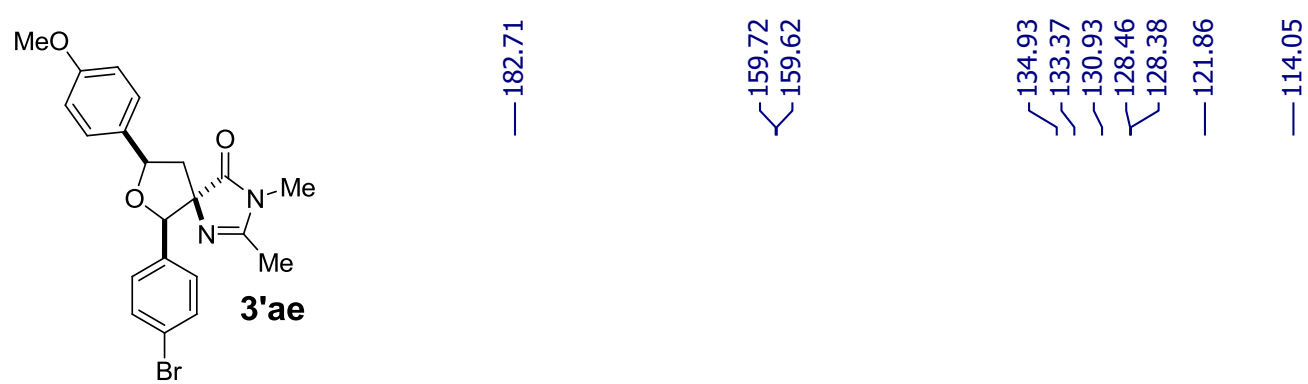

年

l一

${ }^{13} \mathrm{C}$ NMR (175 MHz), $\mathrm{CDCl}_{3}$
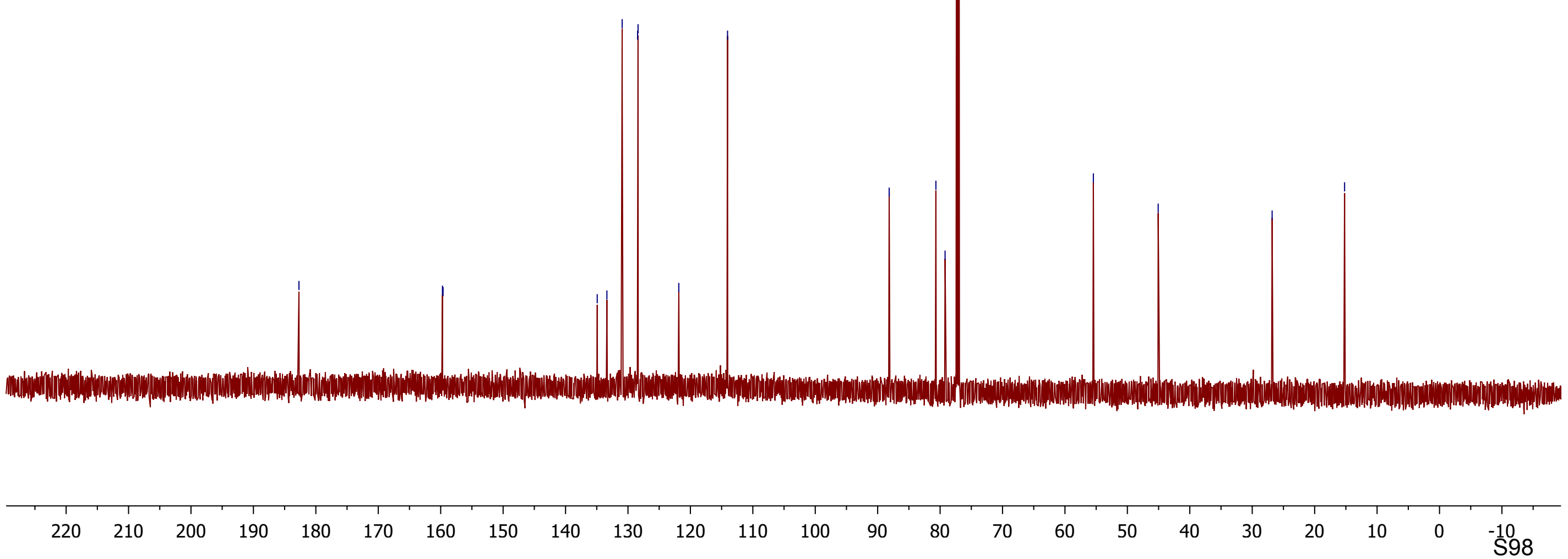


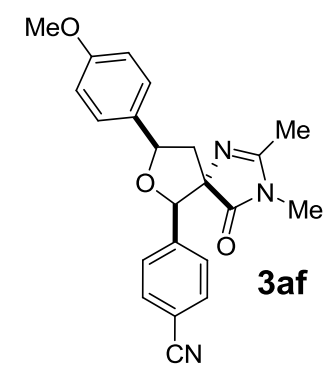

${ }^{1} \mathrm{H}$ NMR (600 MHz), $\mathrm{CDCl}_{3}$

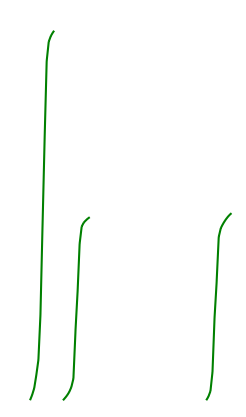

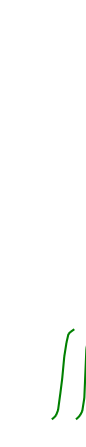

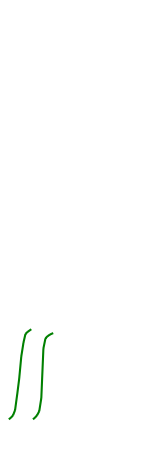

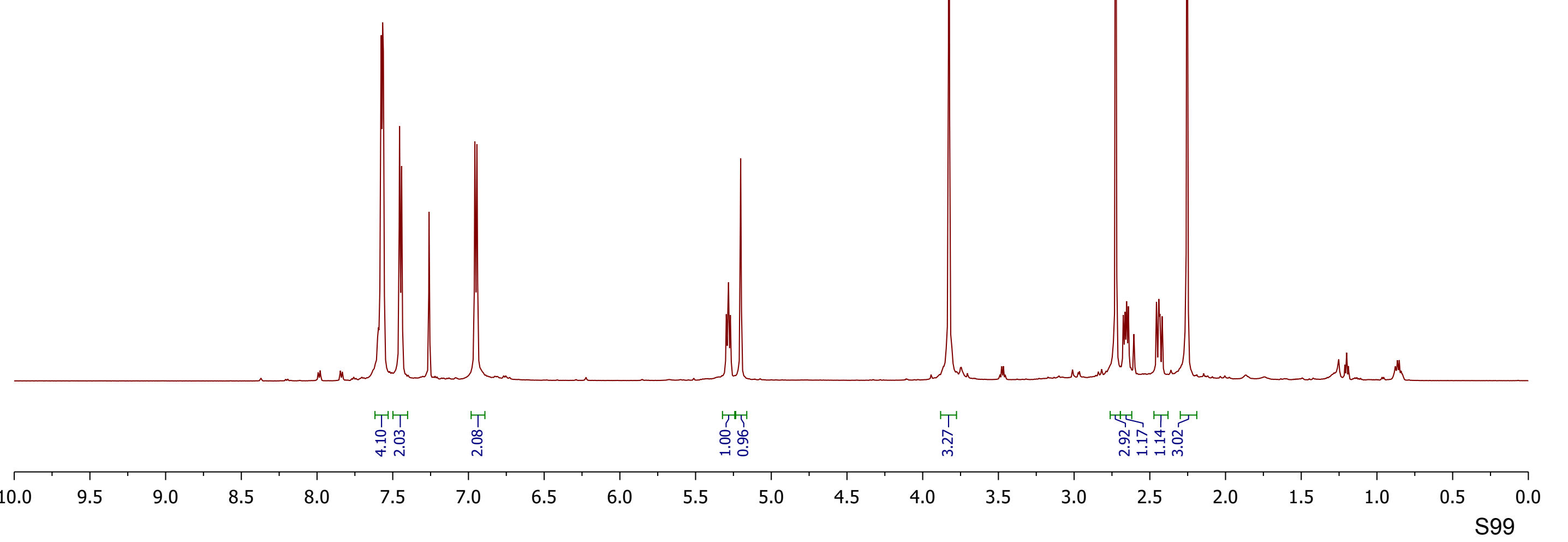




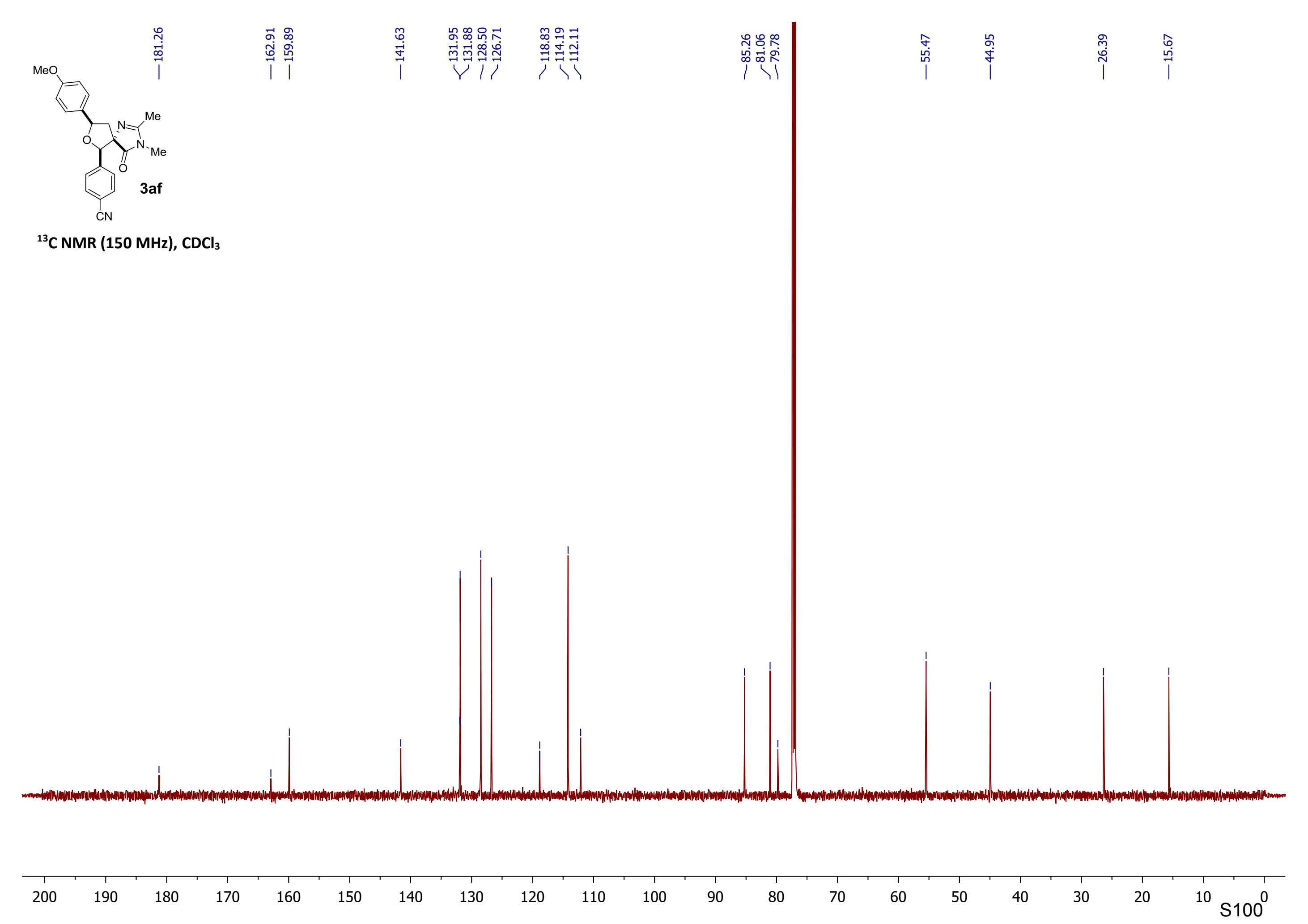




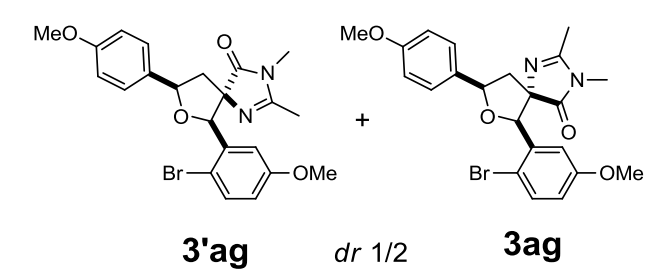

${ }^{1} \mathrm{H}$ NMR (700 MHz), $\mathrm{CDCl}_{3}$

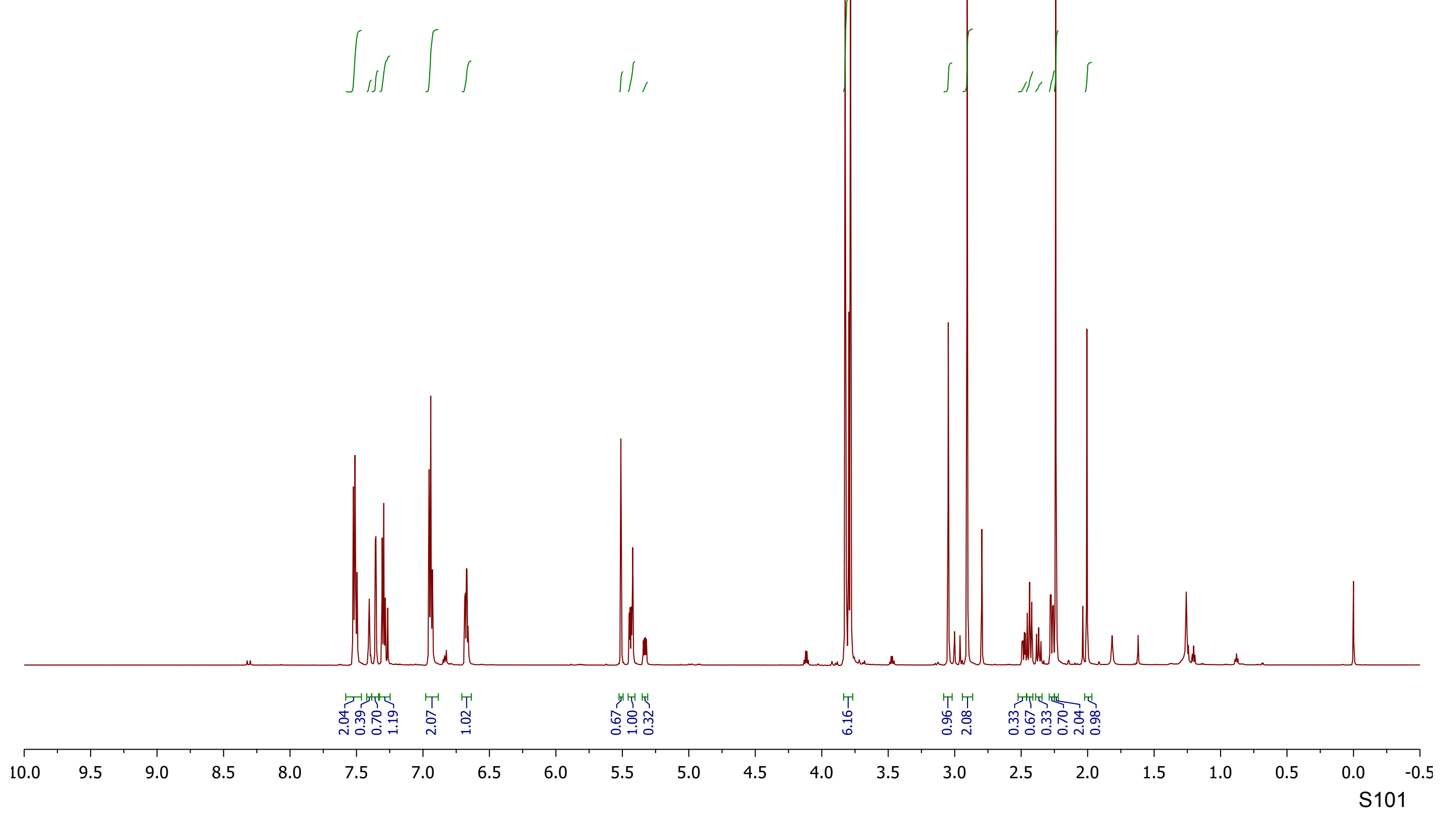


$\mathrm{MeO}$

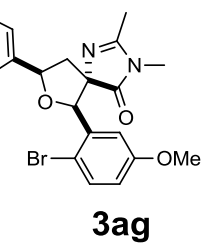

${ }^{13} \mathrm{C}$ NMR (175 MHz), $\mathrm{CDCl}_{3}$ 
HSQC (700 MHz), $\mathrm{CDCl}_{3}$

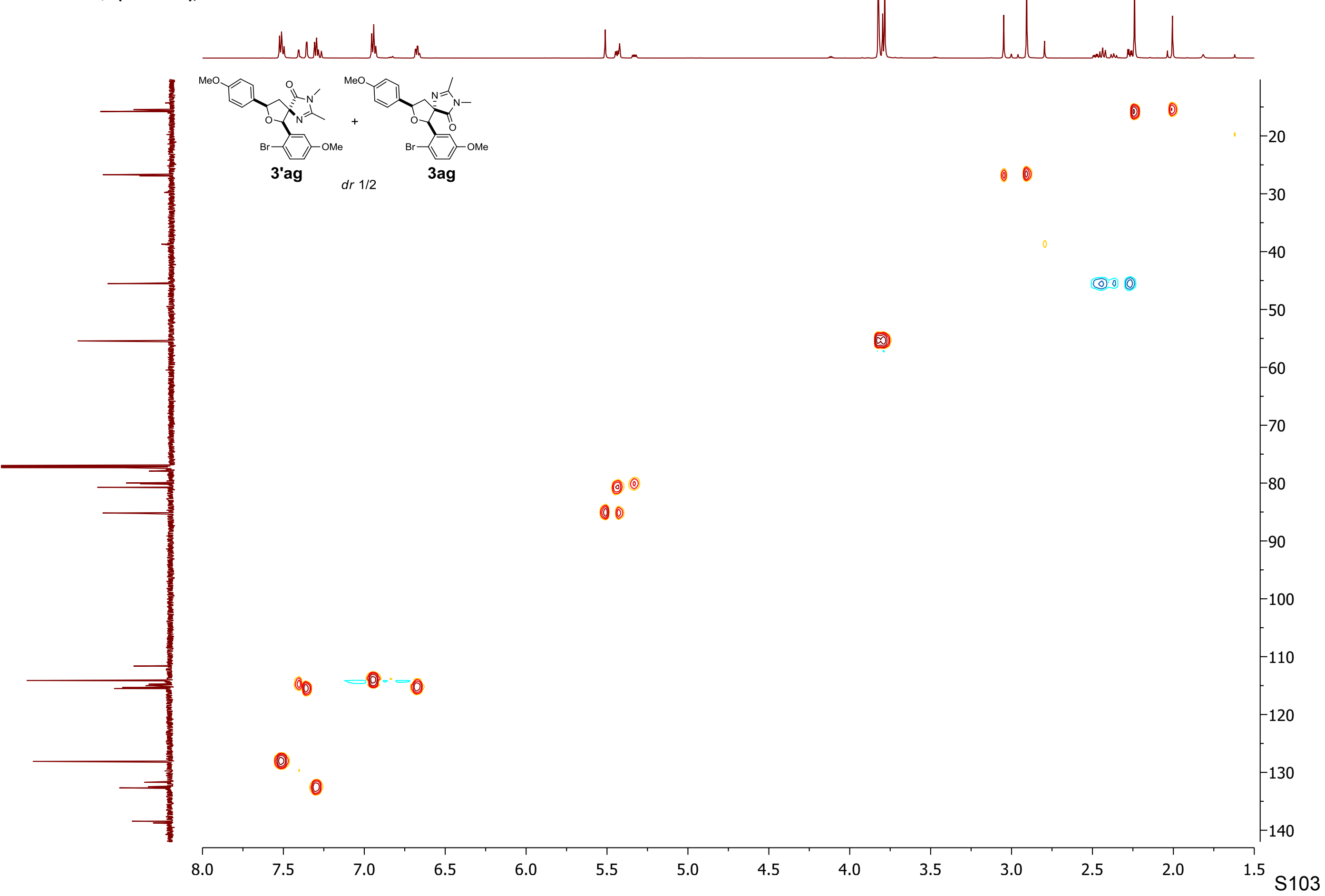




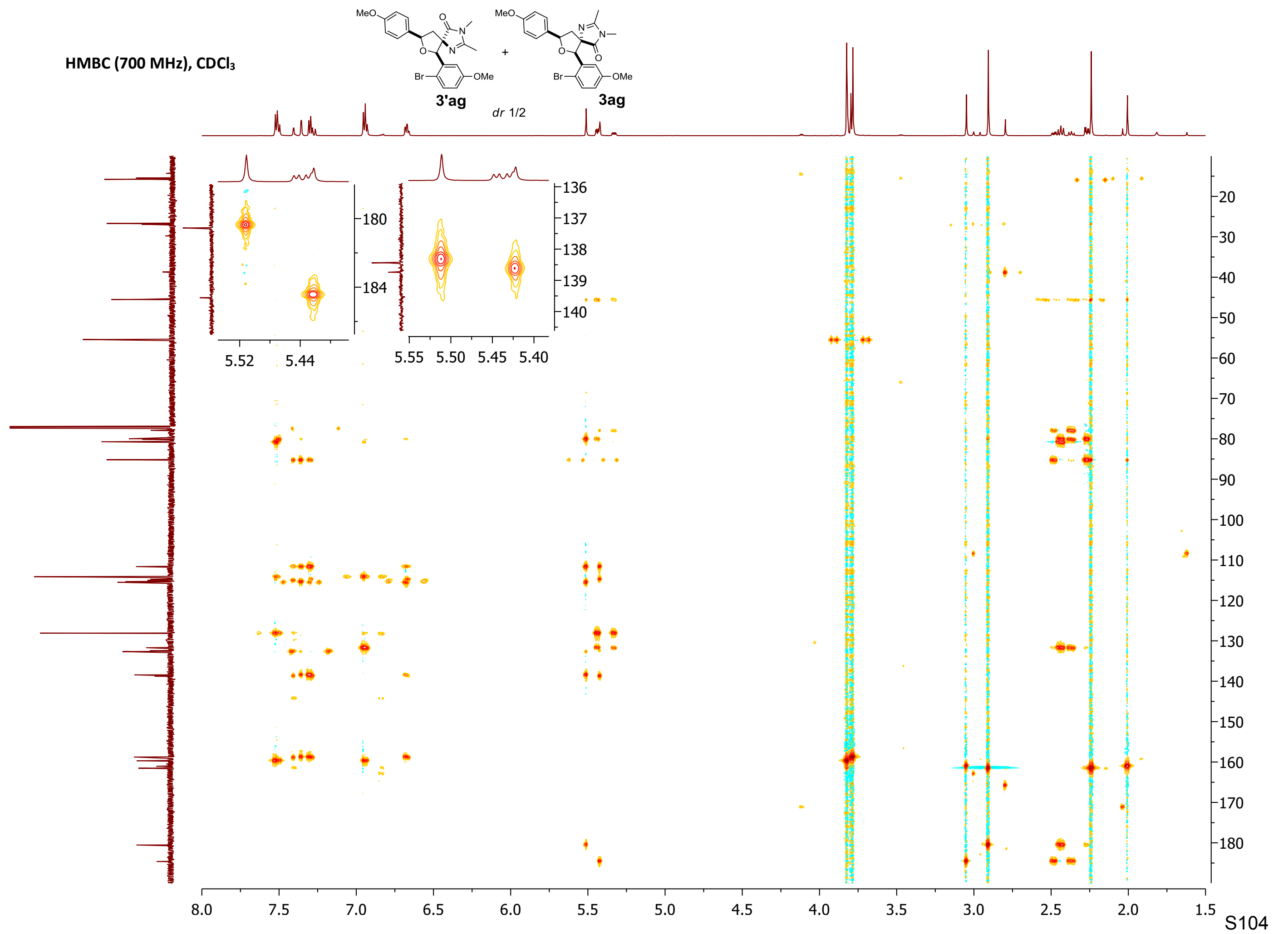




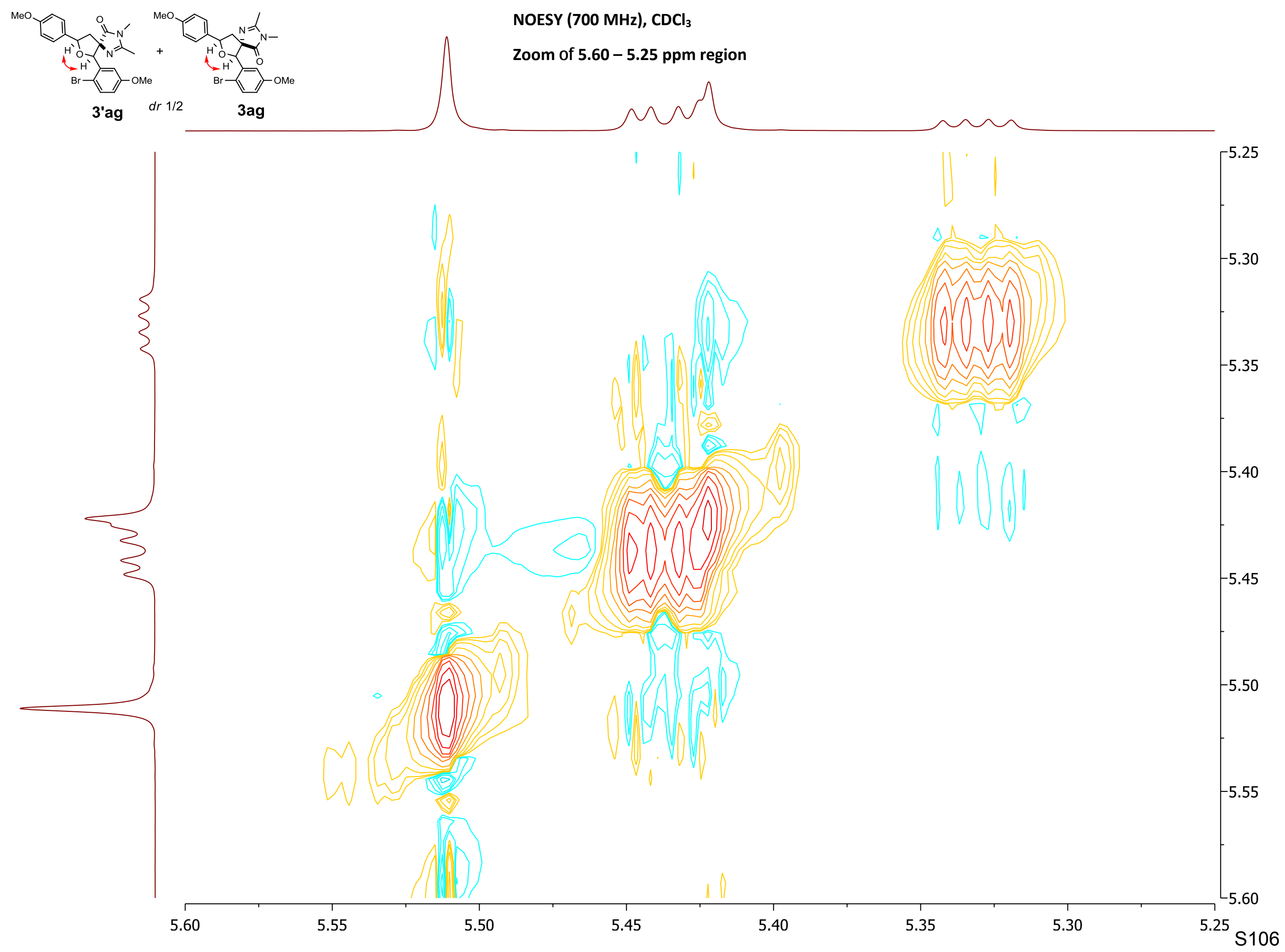




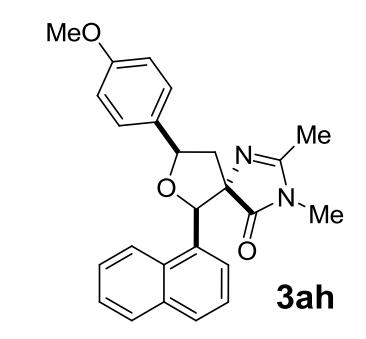

${ }^{1} \mathrm{H}$ NMR (700 MHz), $\mathrm{CDCl}_{3}$
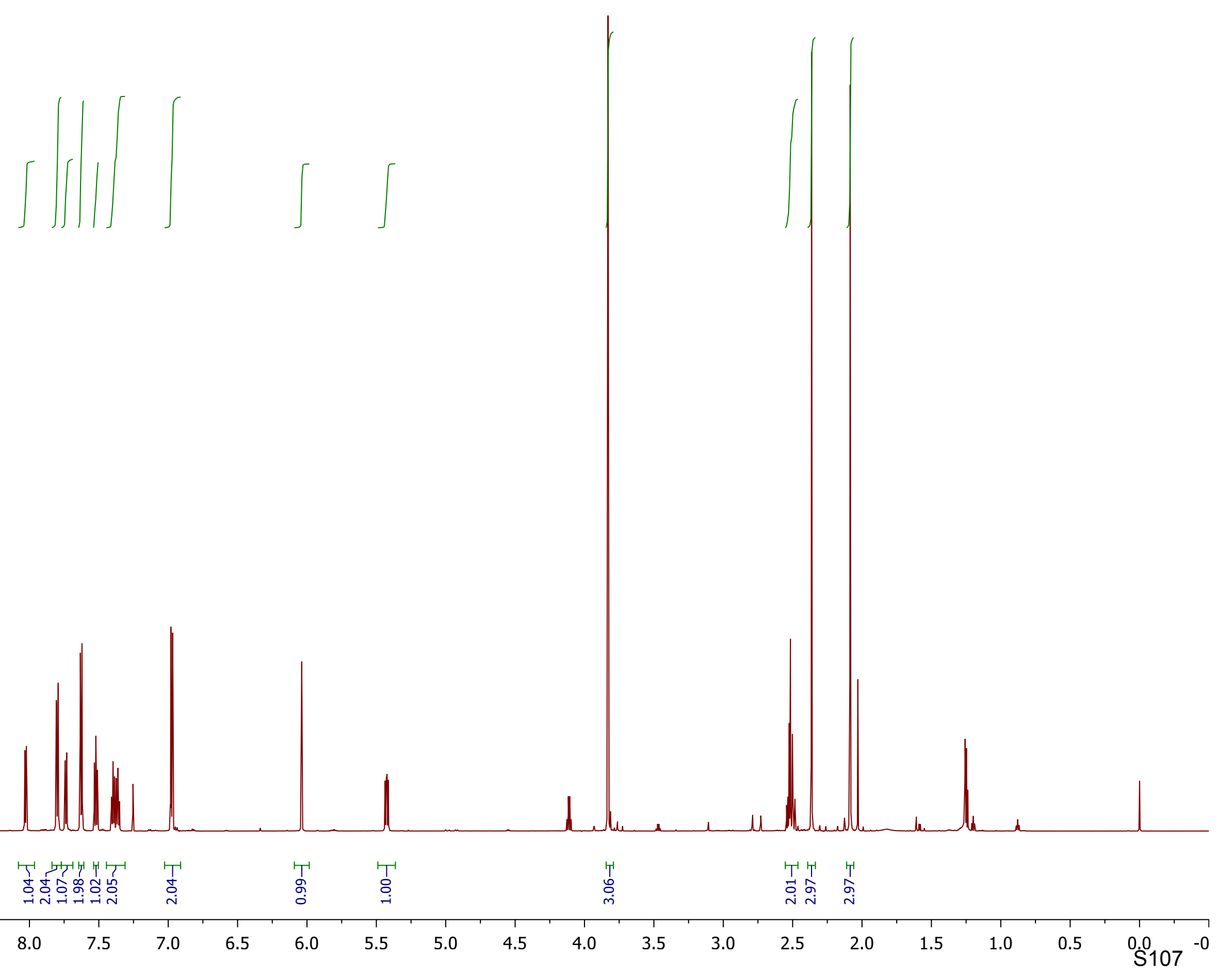

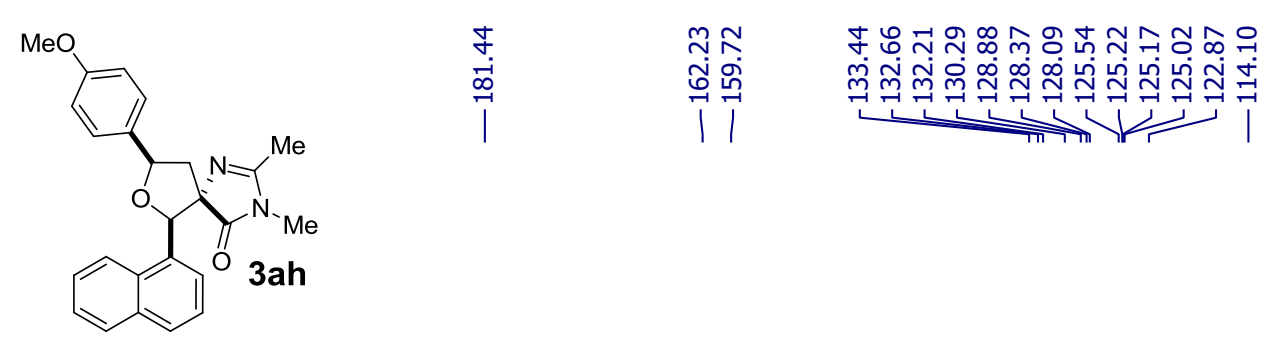

${ }^{13} \mathrm{C}$ NMR (175 MHz), $\mathrm{CDCl}_{3}$

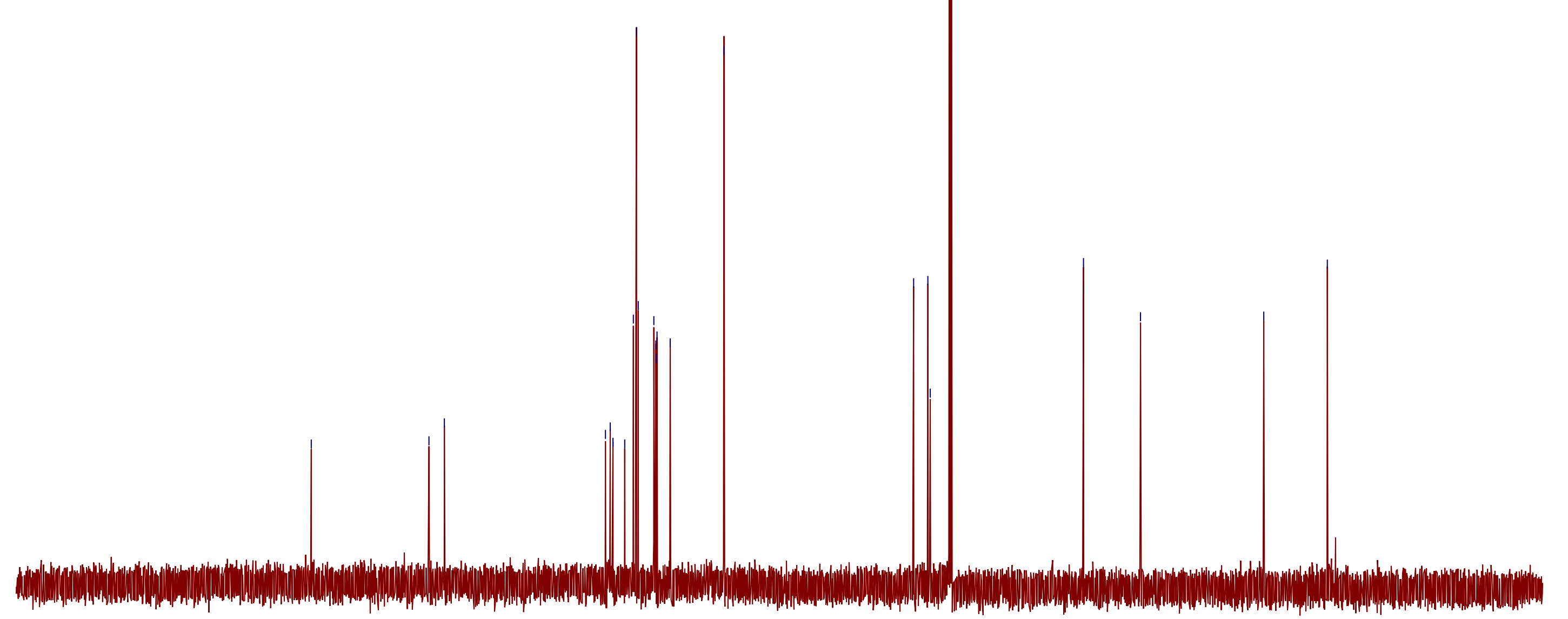




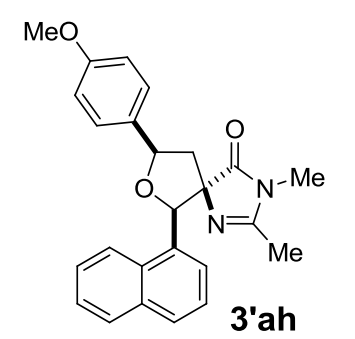

${ }^{1} \mathrm{H}$ NMR (700 MHz), $\mathrm{CDCl}_{3}$
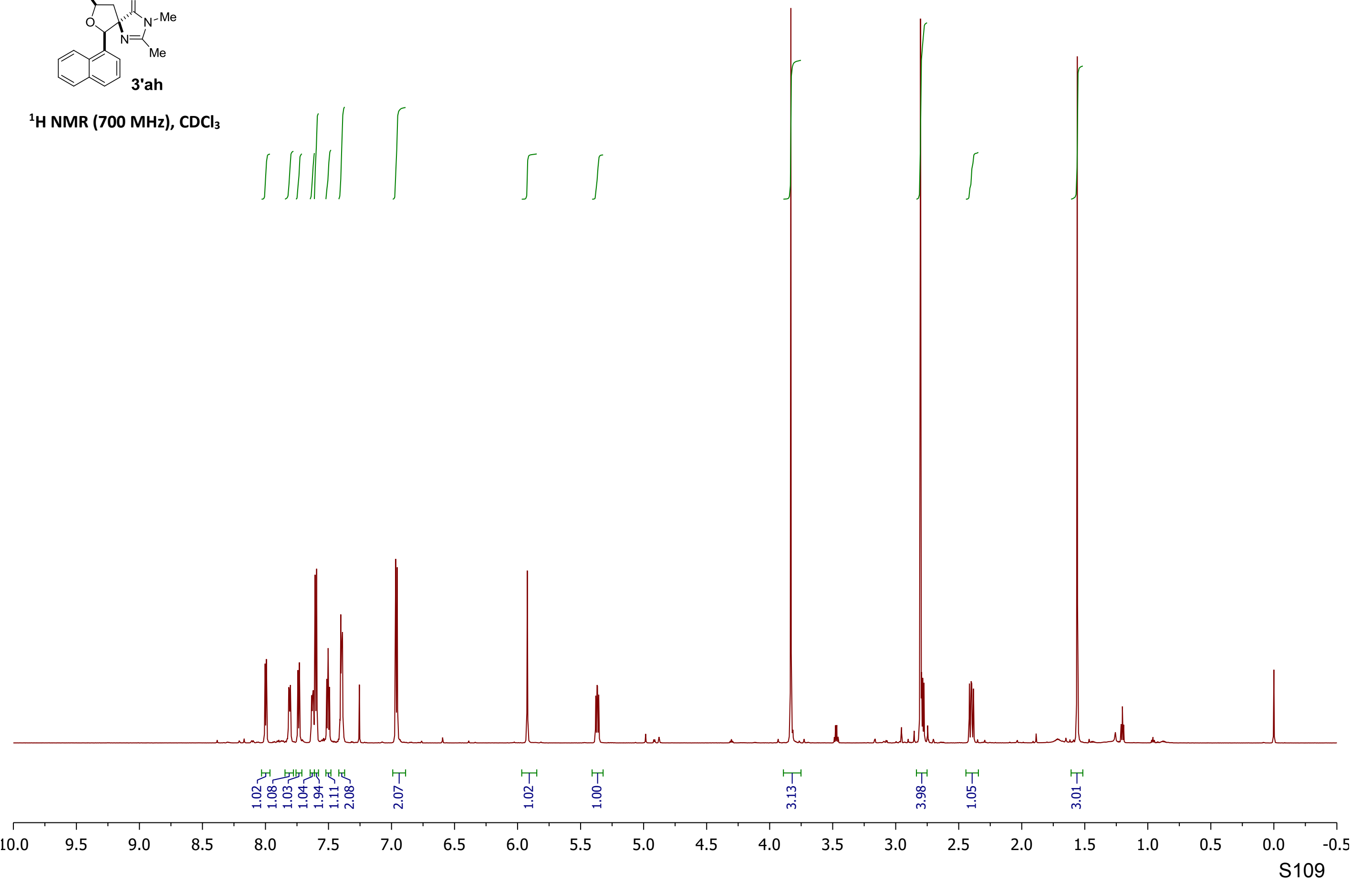


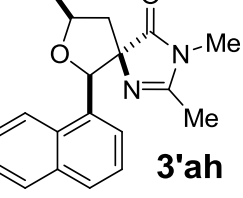

${ }^{13} \mathrm{C}$ NMR (175 MHz), $\mathrm{CDCl}_{3}$

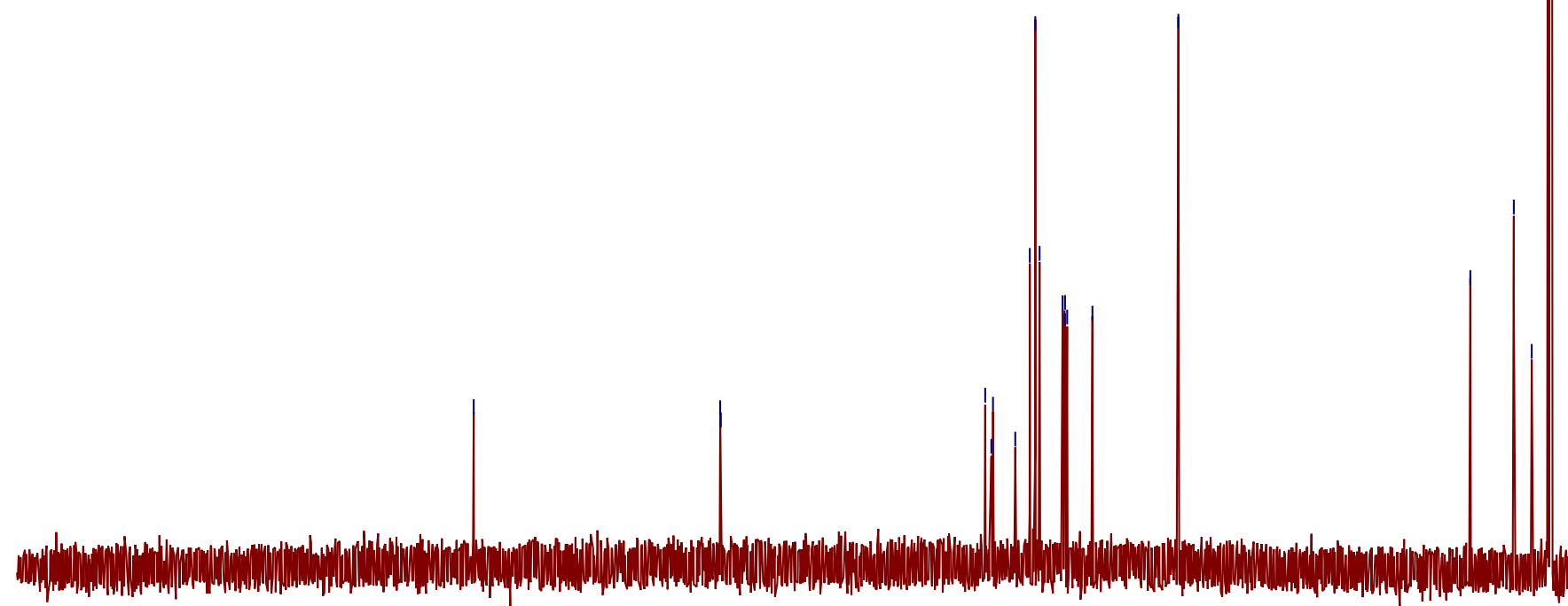

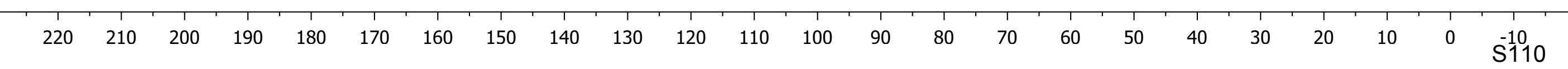


$\mathrm{MeO}$

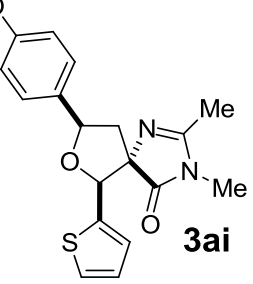

${ }^{1} \mathrm{H}_{\mathrm{NMR}}(700 \mathrm{MHz}), \mathrm{CDCl}_{3}$
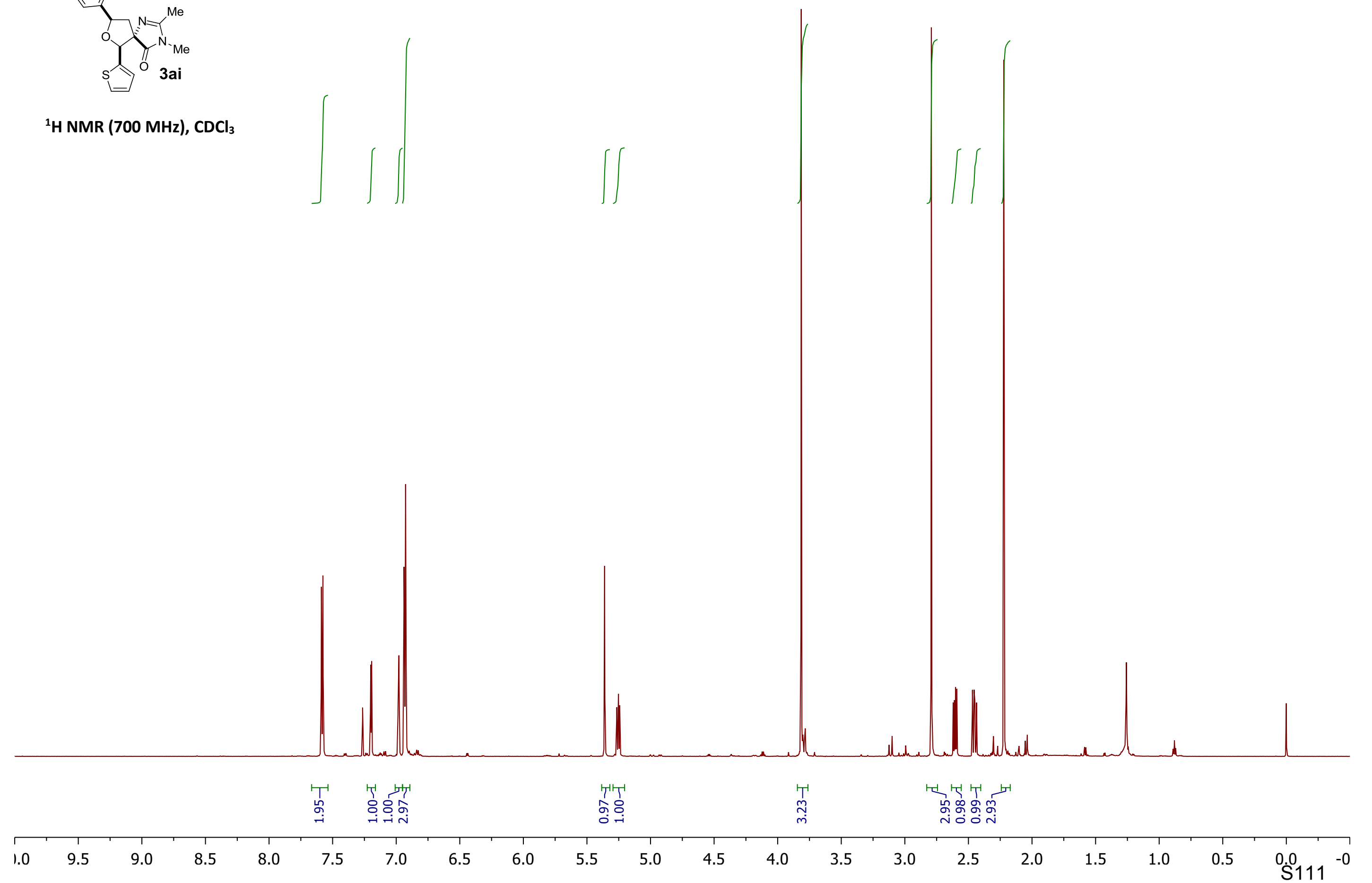

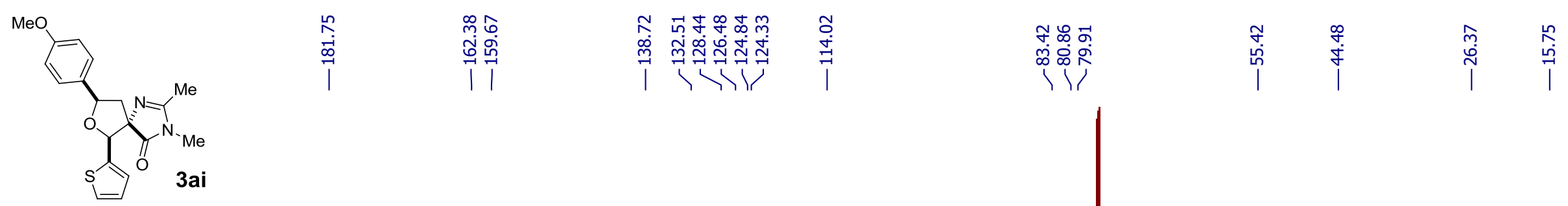

${ }^{13} \mathrm{C}$ NMR (175 MHz), $\mathrm{CDCl}_{3}$
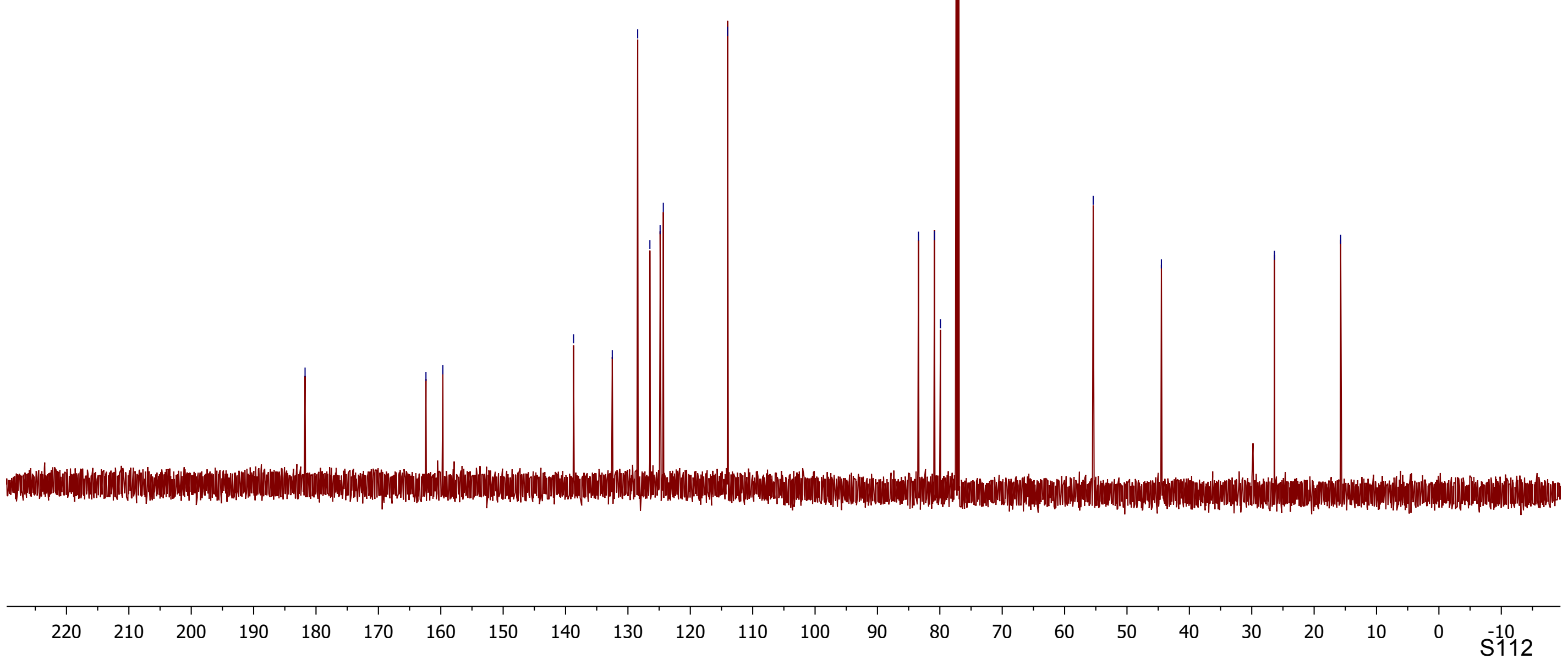


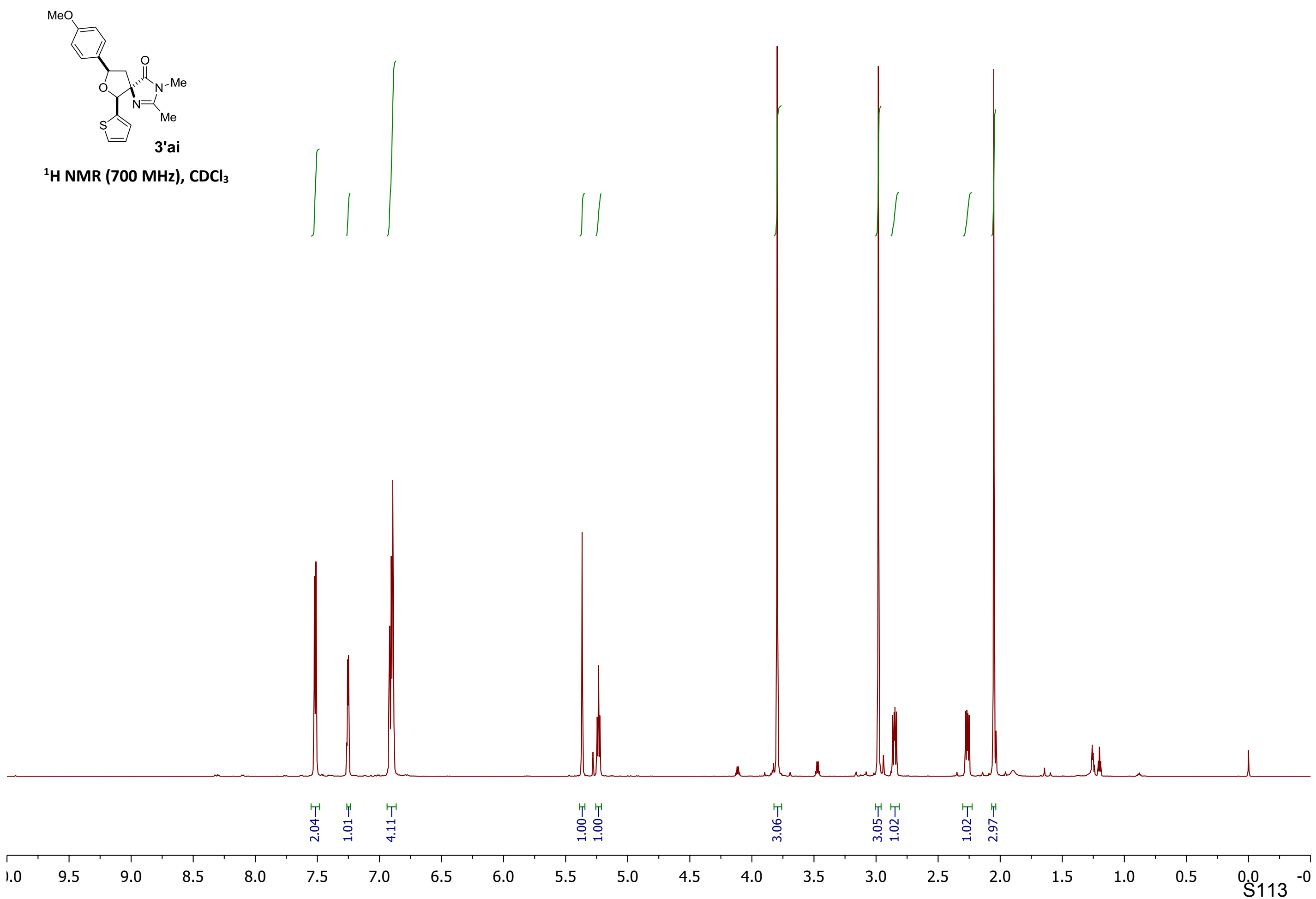




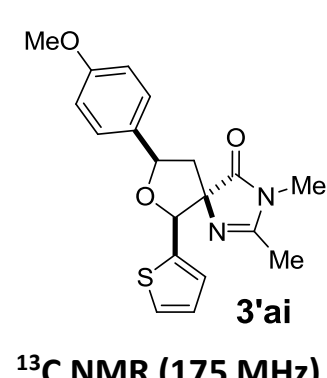

${ }^{13} \mathrm{C}$ NMR (175 MHz), $\mathrm{CDCl}_{3}$

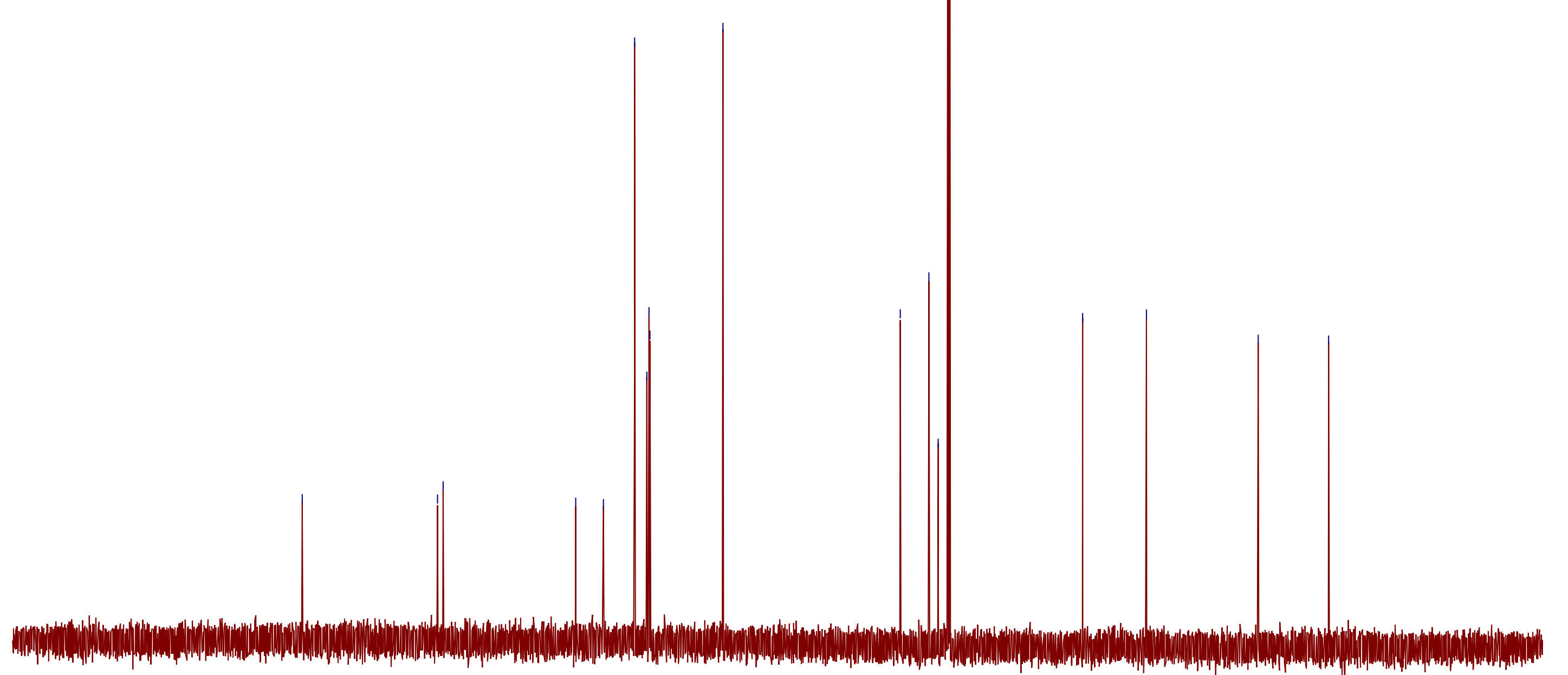

\begin{tabular}{lllllllllllllllllllllllll}
\hline 220 & 210 & 200 & 190 & 180 & 170 & 160 & 150 & 140 & 130 & 120 & 110 & 100 & 90 & 80 & 70 & 60 & 50 & 40 & 30 & 20 & 10 & 0 & -10 &
\end{tabular}




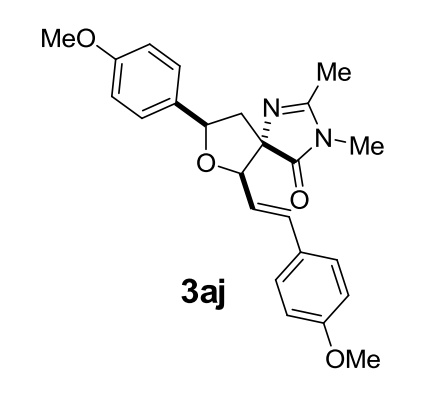

${ }^{1} \mathrm{H} \mathrm{NMR}(700 \mathrm{MHz}), \mathrm{CDCl}_{3}$

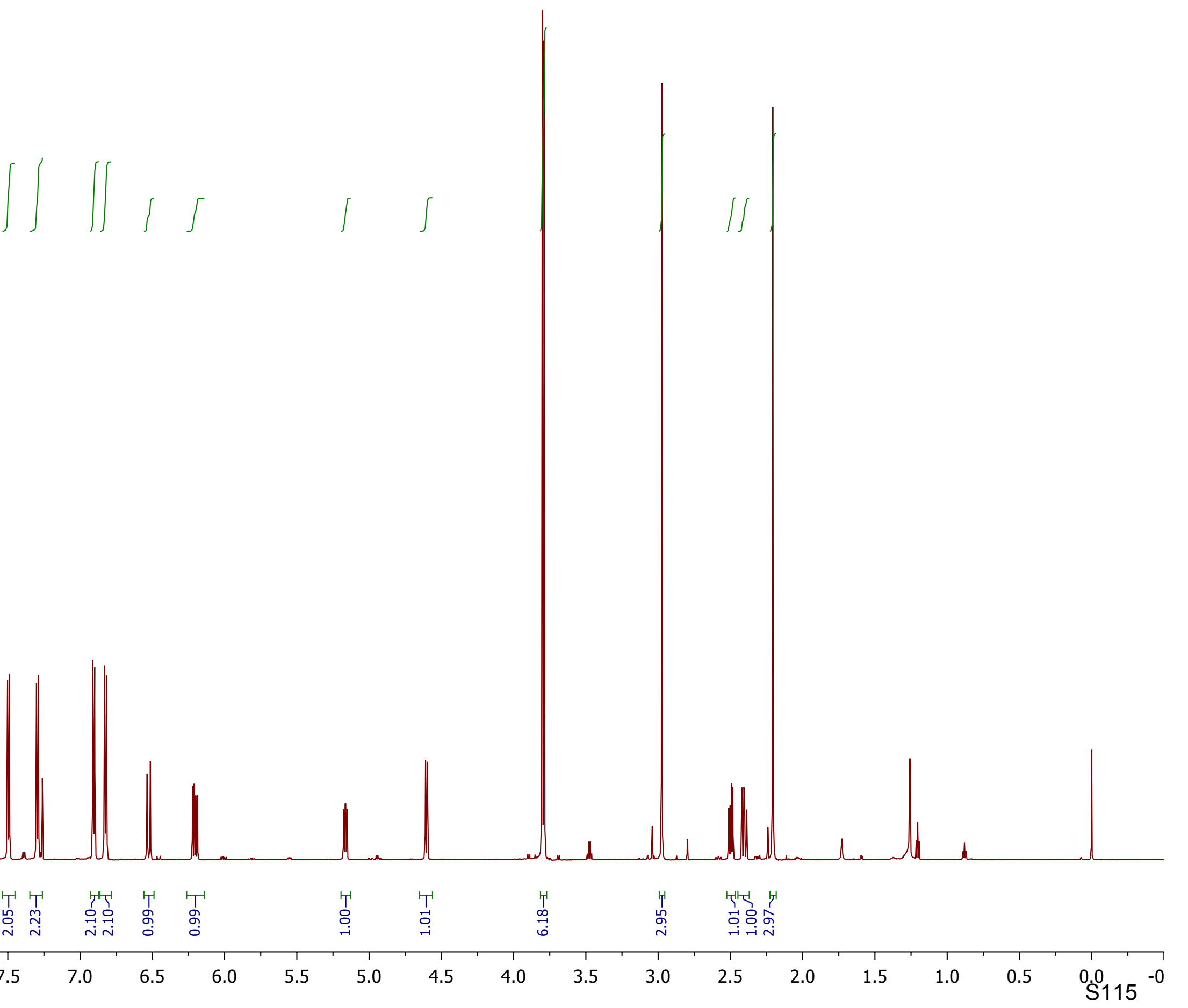




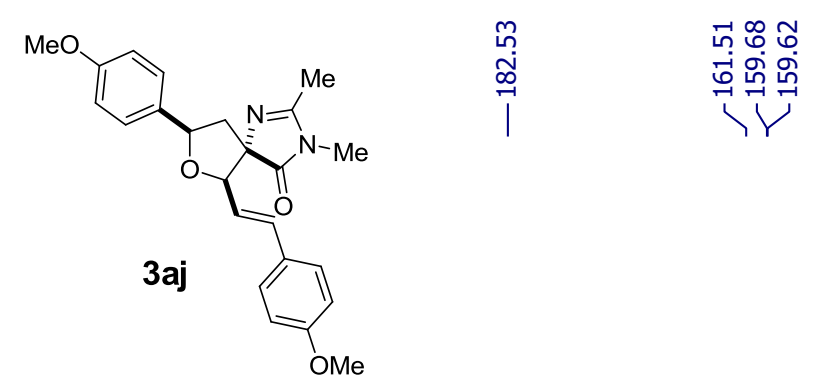

${ }^{13} \mathrm{C} \mathrm{NMR} \mathrm{(175} \mathrm{MHz),} \mathrm{CDCl}_{3}$

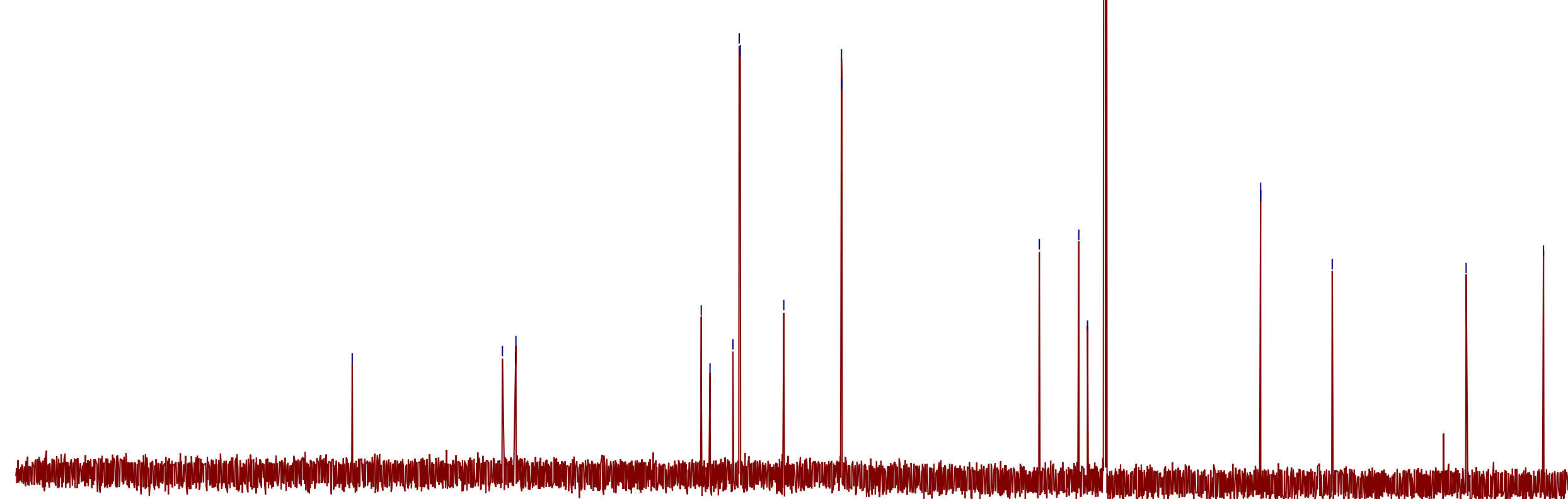

\begin{tabular}{llllllllllllllllllllllllllllll}
\hline 220 & 210 & 200 & 190 & 180 & 170 & 160 & 150 & 140 & 130 & 120 & 110 & 100 & 90 & 80 & 70 & 60 & 50 & 40 & 30 & 20 & 10 & 0 & -10 &
\end{tabular}




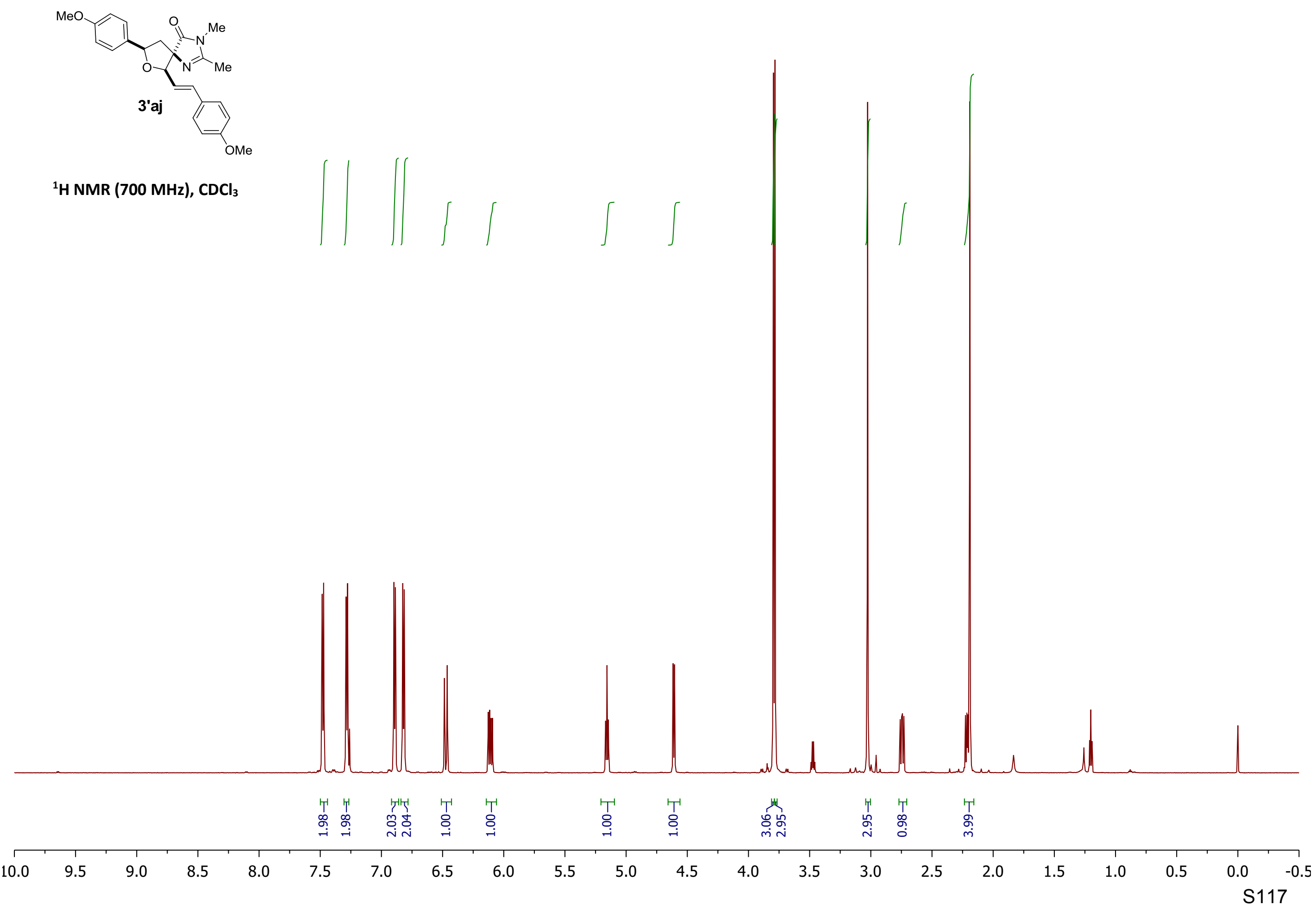




$$
\text { 等 }
$$


$\mathrm{MeO}$

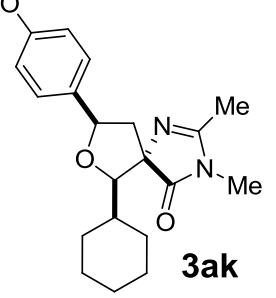

${ }^{1} \mathrm{H}$ NMR (700 MHz), $\mathrm{CDCl}_{3}$
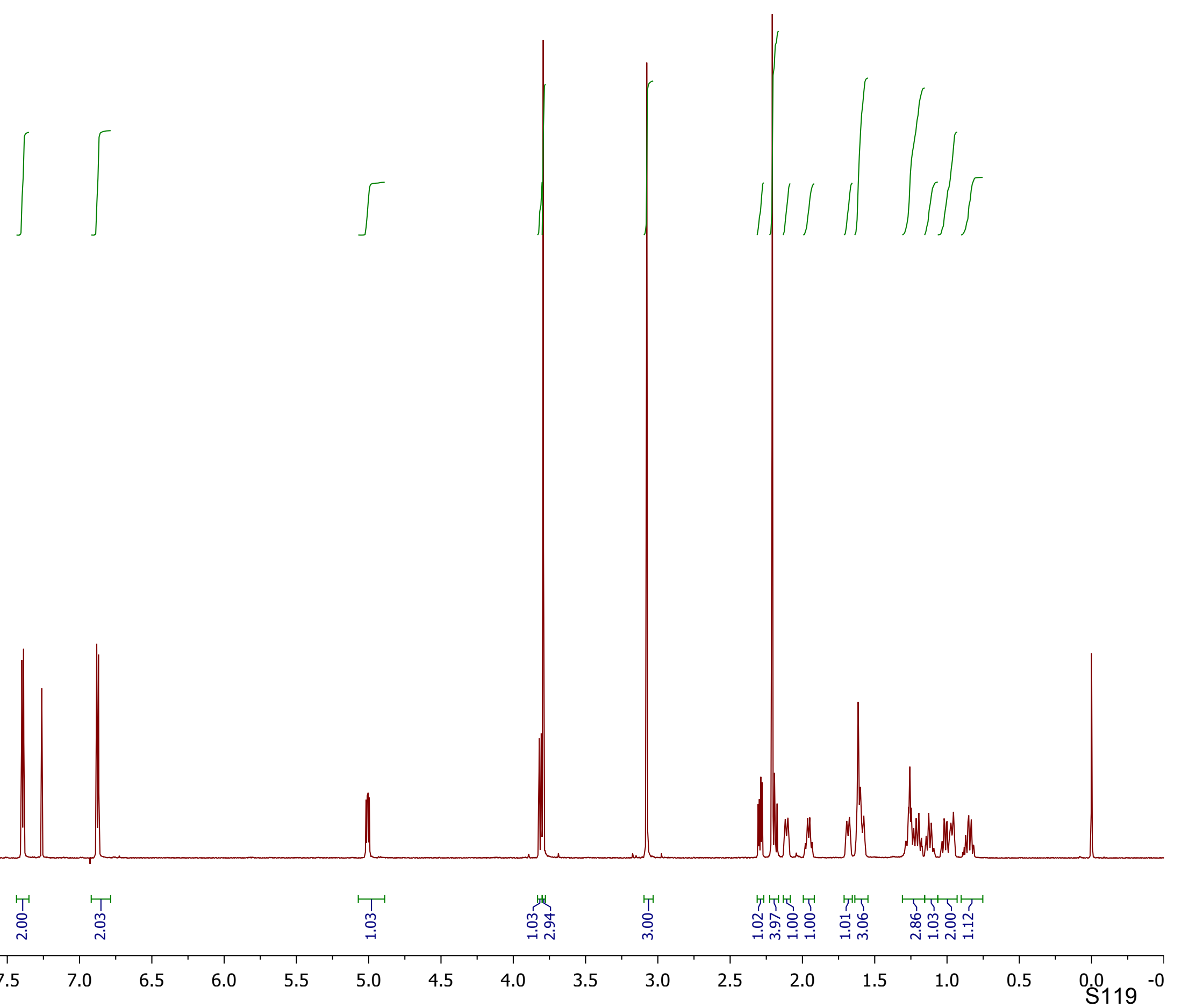

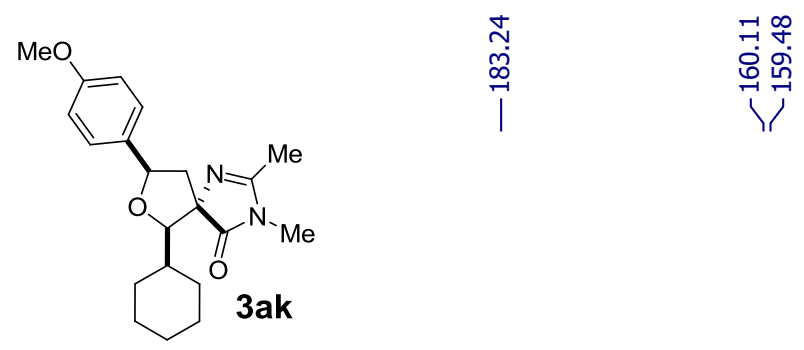

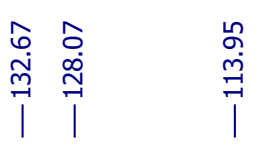

|

${ }^{13} \mathrm{C}$ NMR (175 MHz), $\mathrm{CDCl}_{3}$
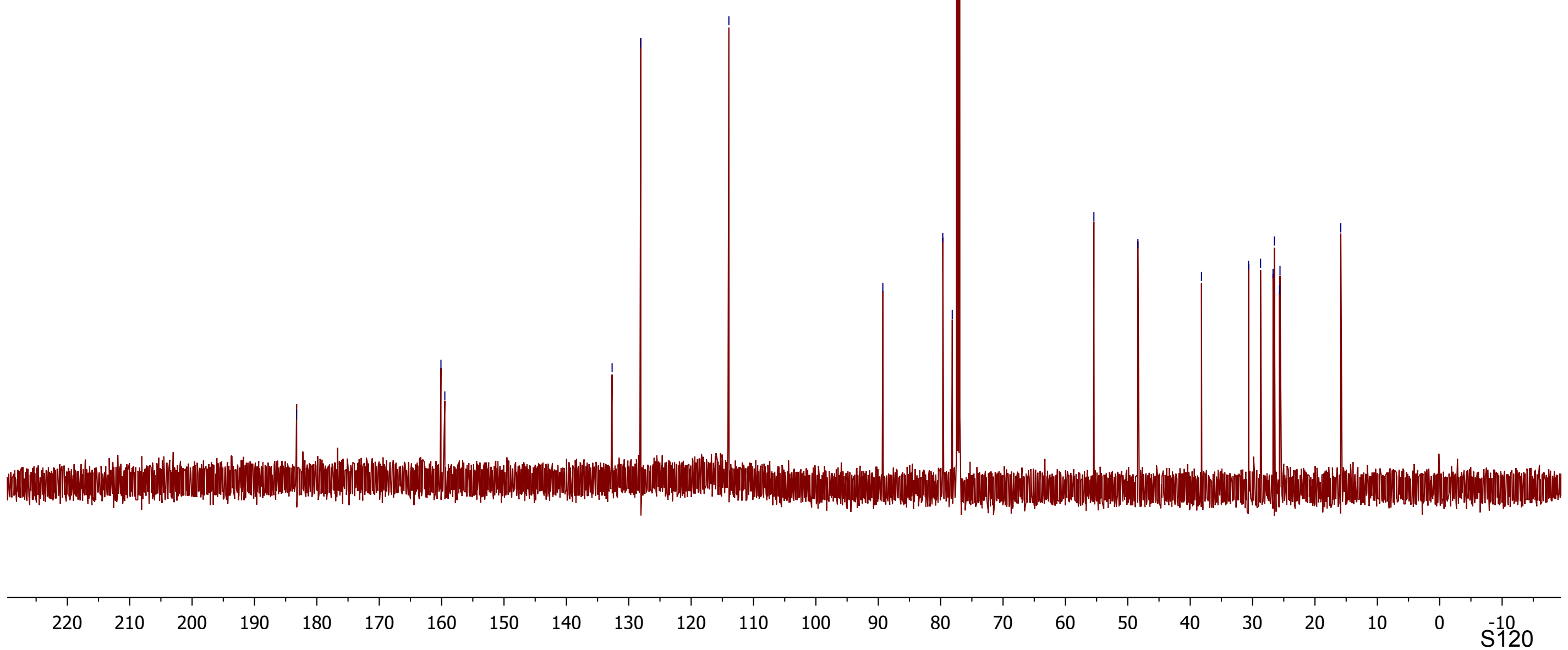
$\mathrm{MeO}$

$$
\overbrace{3 \text { ak }}^{2}
$$

${ }^{1} \mathrm{H}$ NMR (700 MHz), $\mathrm{CDCl}_{3}$

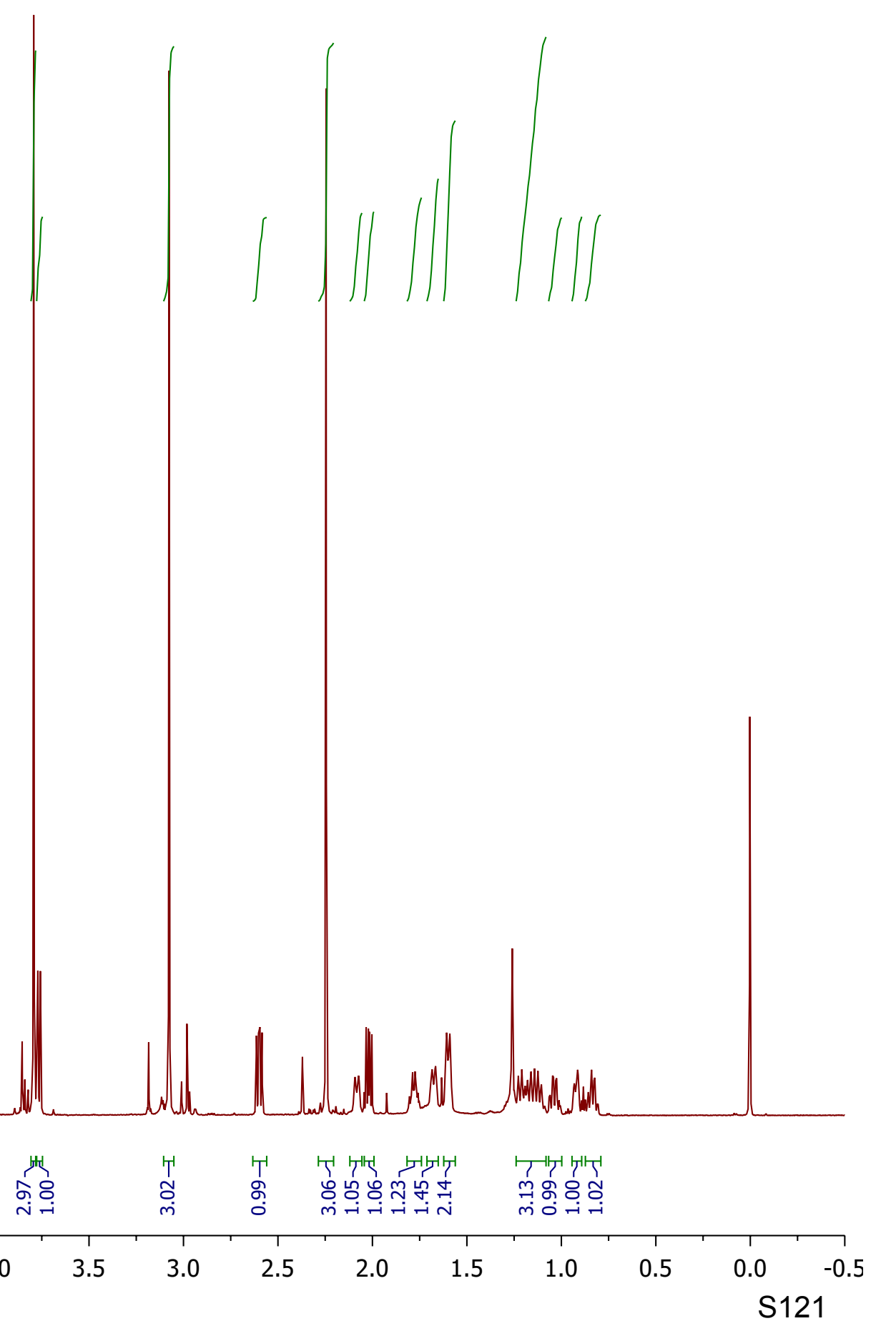




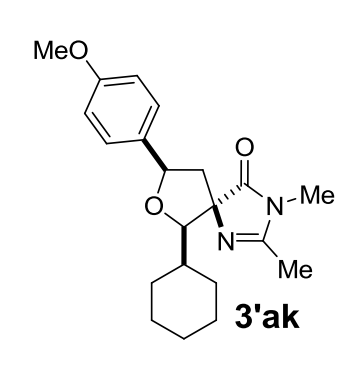

${ }^{13} \mathrm{C}$ NMR (175 MHz), $\mathrm{CDCl}_{3}$

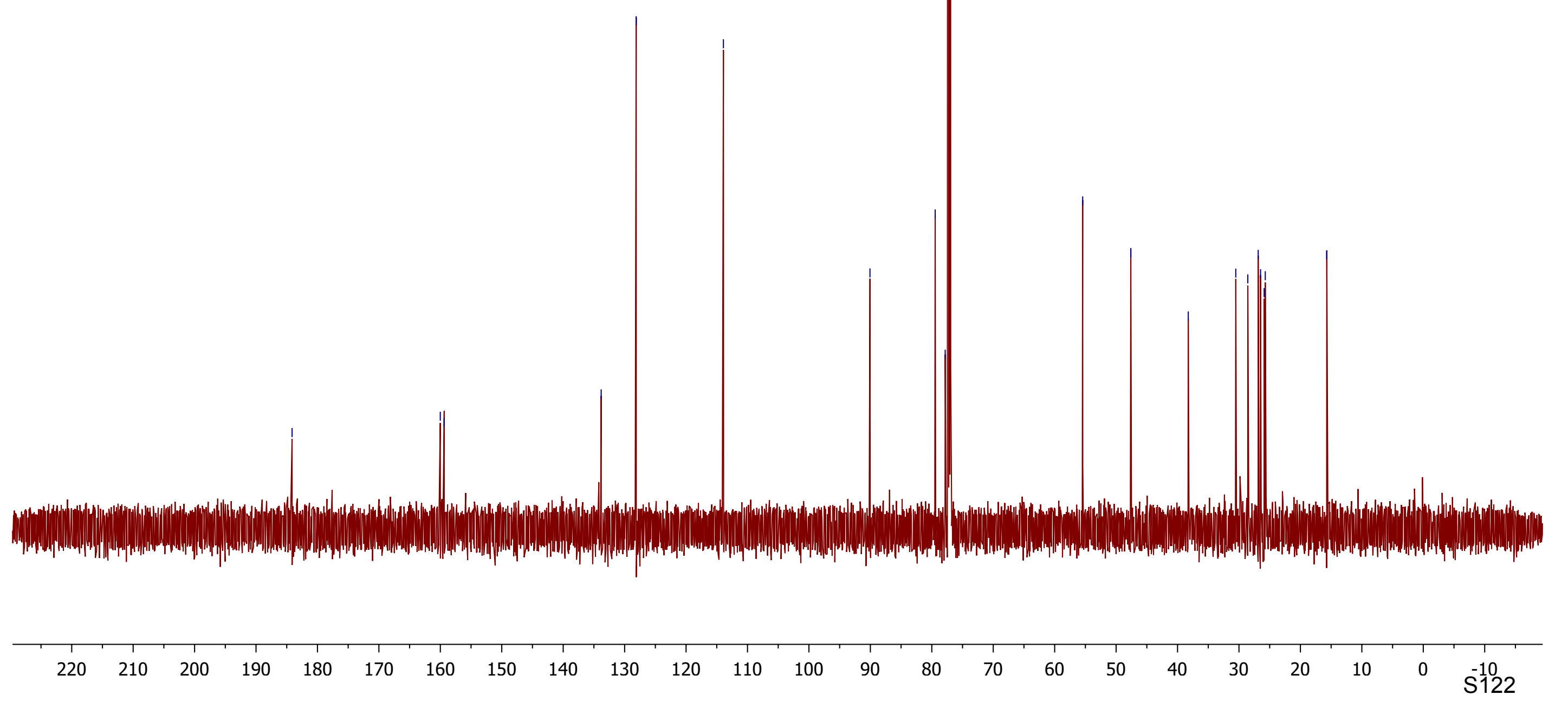




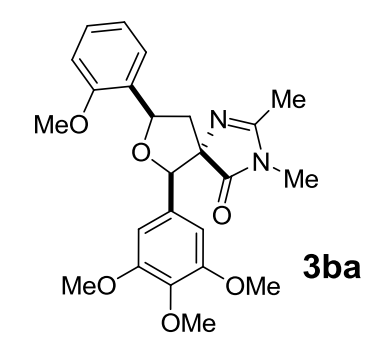

${ }^{1} \mathrm{H}$ NMR (700 MHz), $\mathrm{CDCl}_{3}$

,

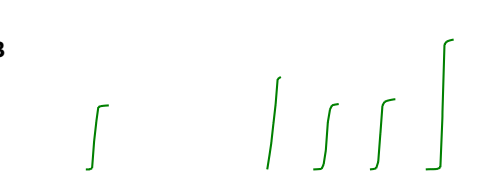

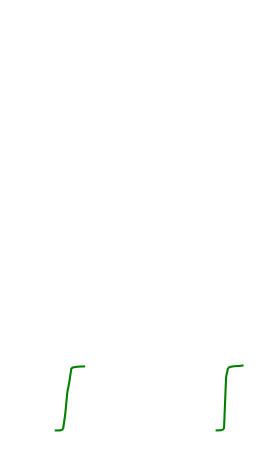
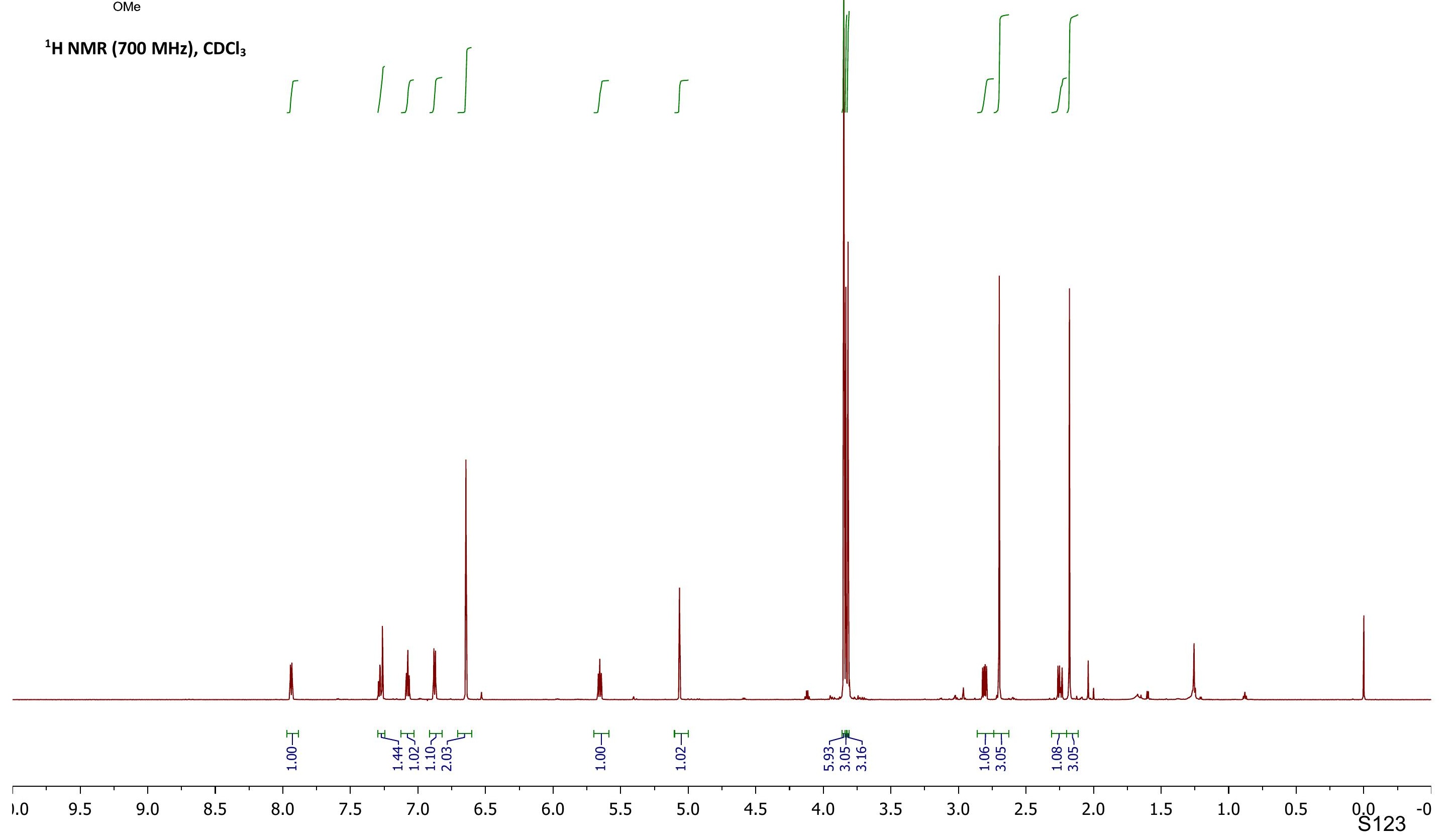



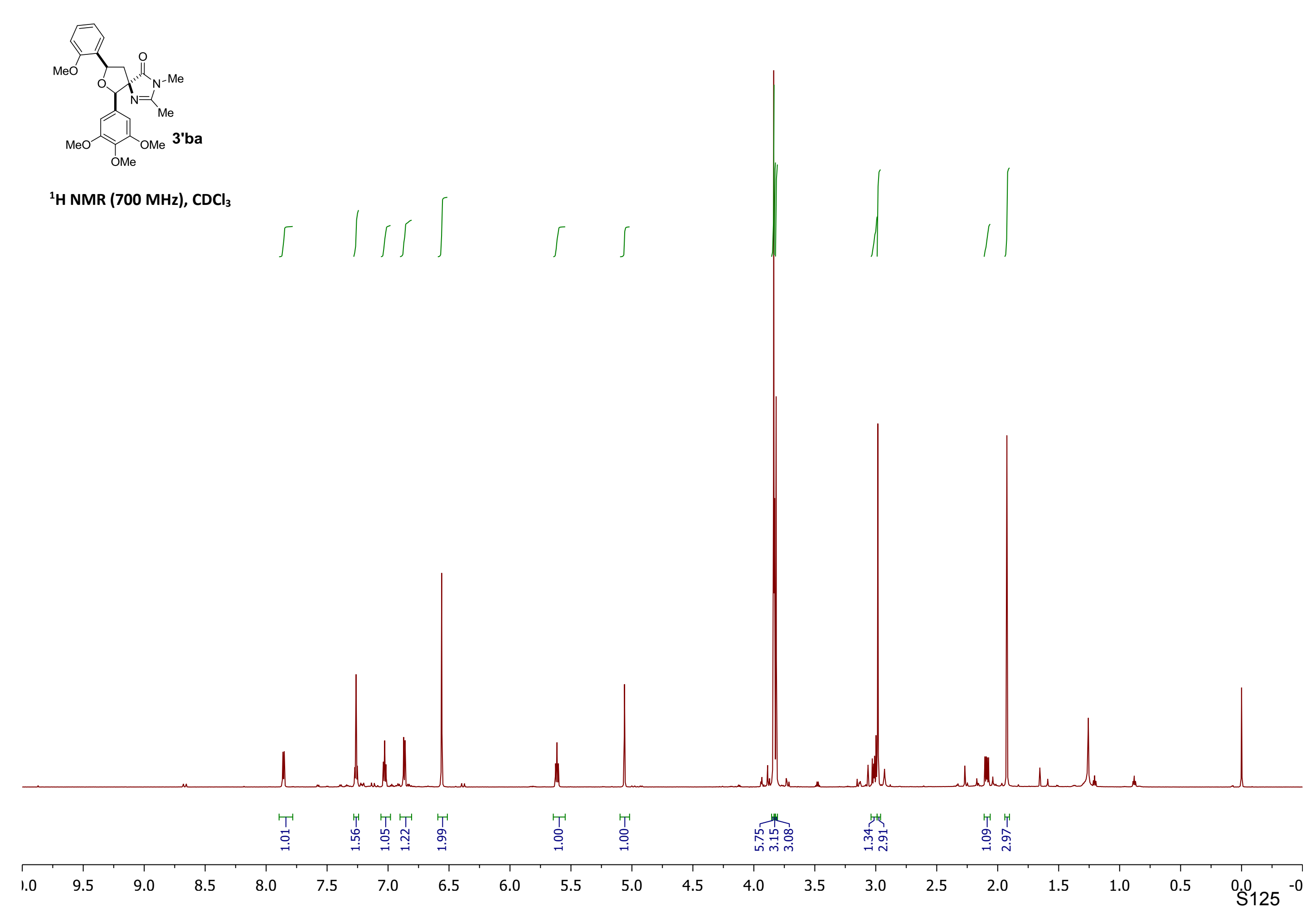




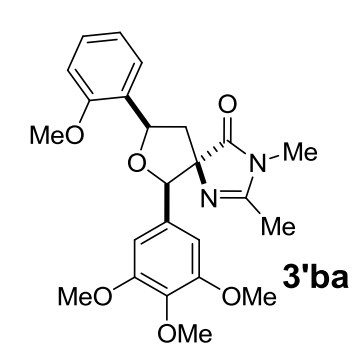

${ }^{13} \mathrm{C} \mathrm{NMR} \mathrm{(75} \mathrm{MHz),} \mathrm{CDCl}_{3}$

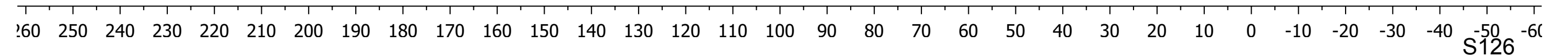




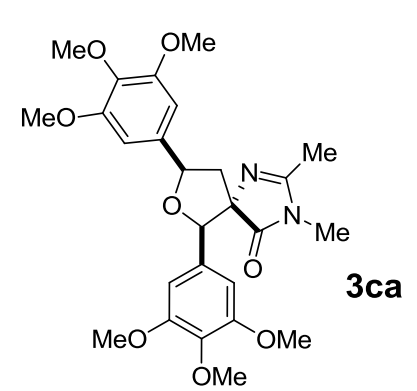

${ }^{1} \mathrm{H}$ NMR (700 MHz), $\mathrm{CDCl}_{3}$

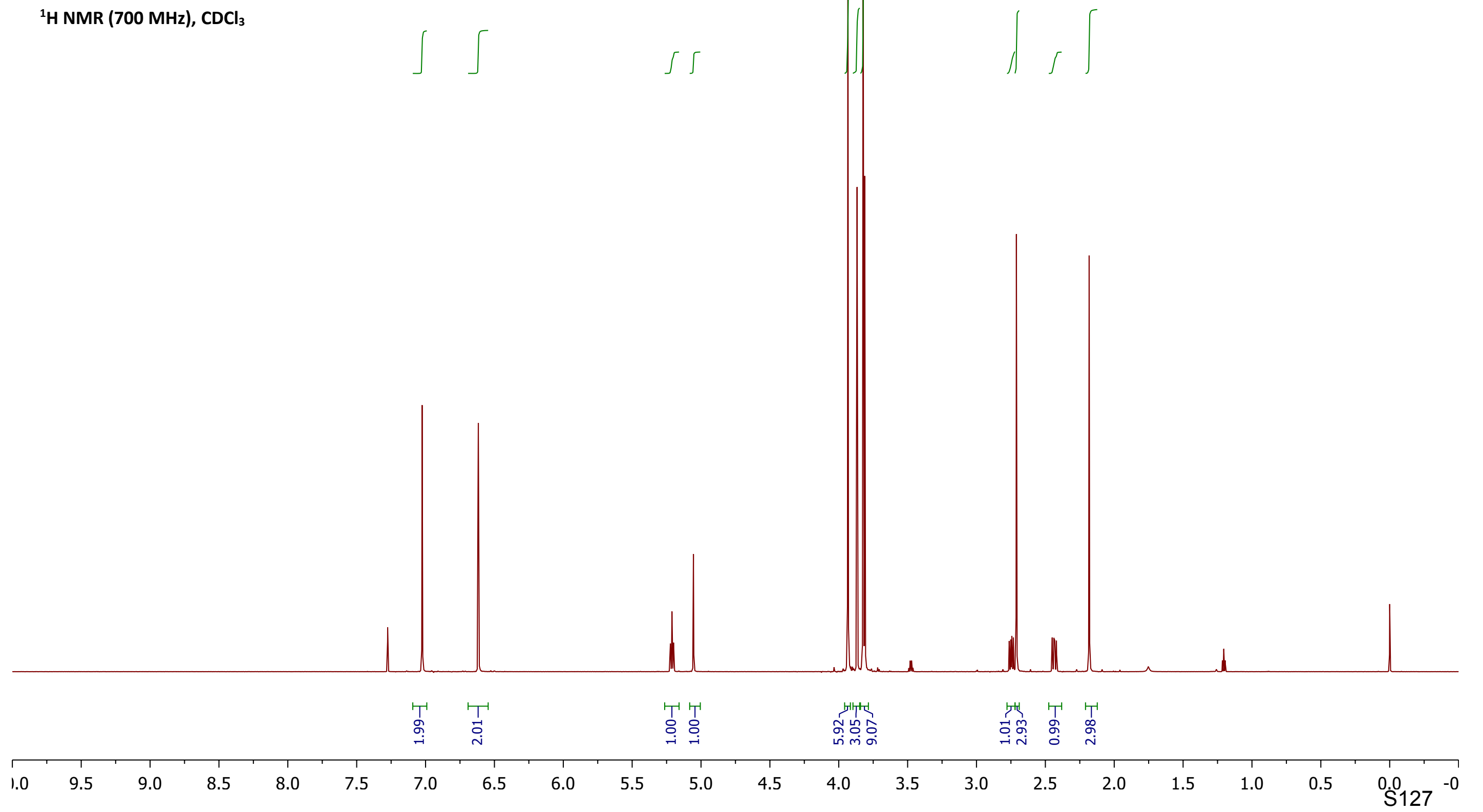




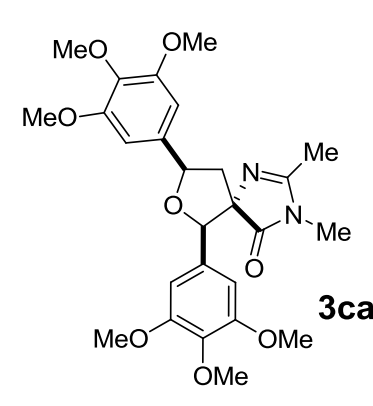

${ }^{13} \mathrm{C}$ NMR (175 MHz), $\mathrm{CDCl}_{3}$

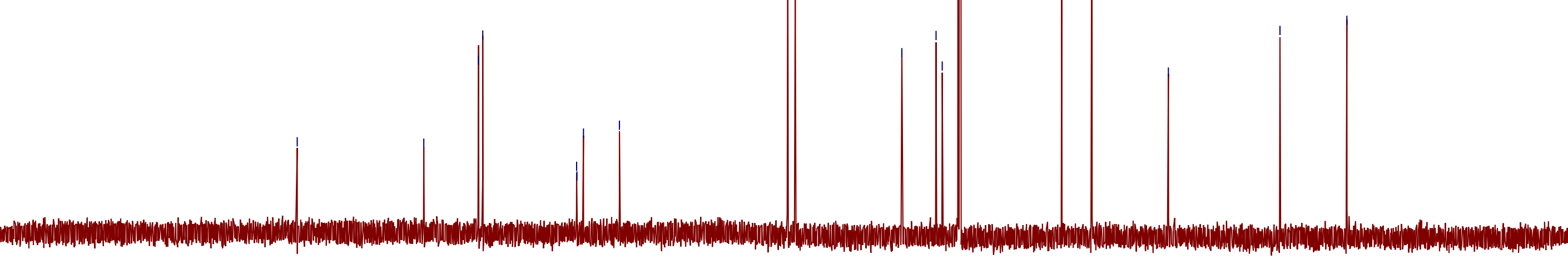




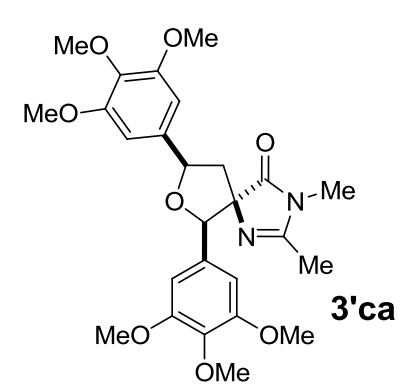

${ }^{1} \mathrm{H}$ NMR (700 MHz), $\mathrm{CDCl}_{3}$ 


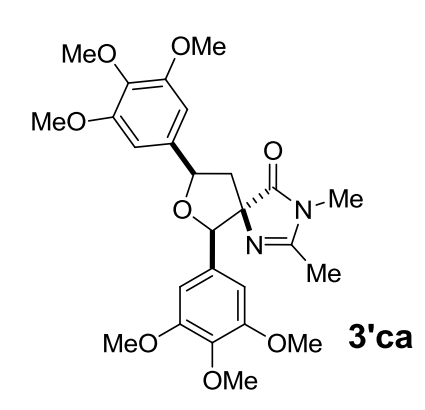

${ }^{13} \mathrm{C}$ NMR $(75 \mathrm{MHz}), \mathrm{CDCl}_{3}$

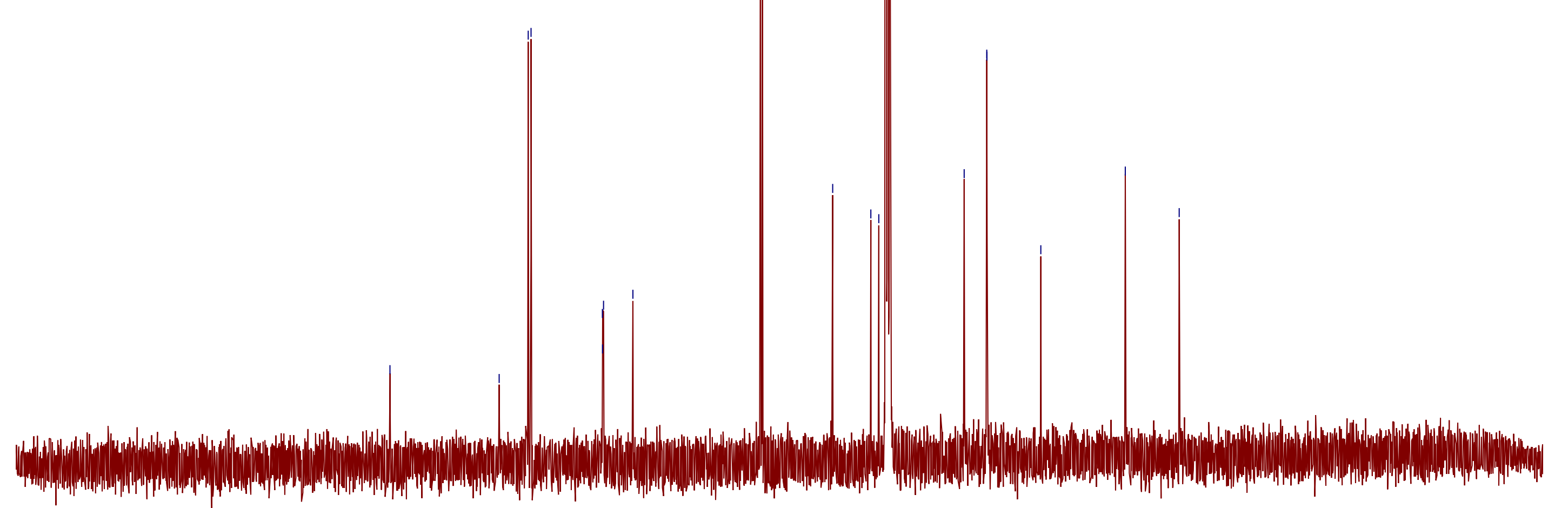

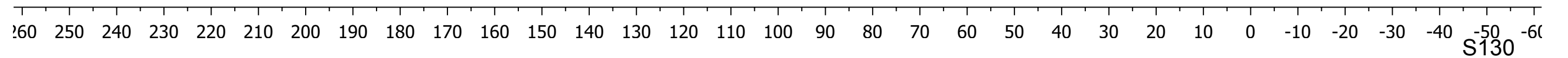




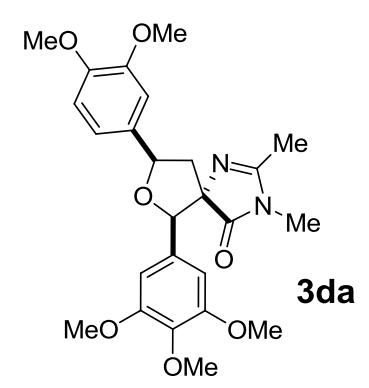

${ }^{1} \mathrm{H}$ NMR (700 MHz), $\mathrm{CDCl}_{3}$

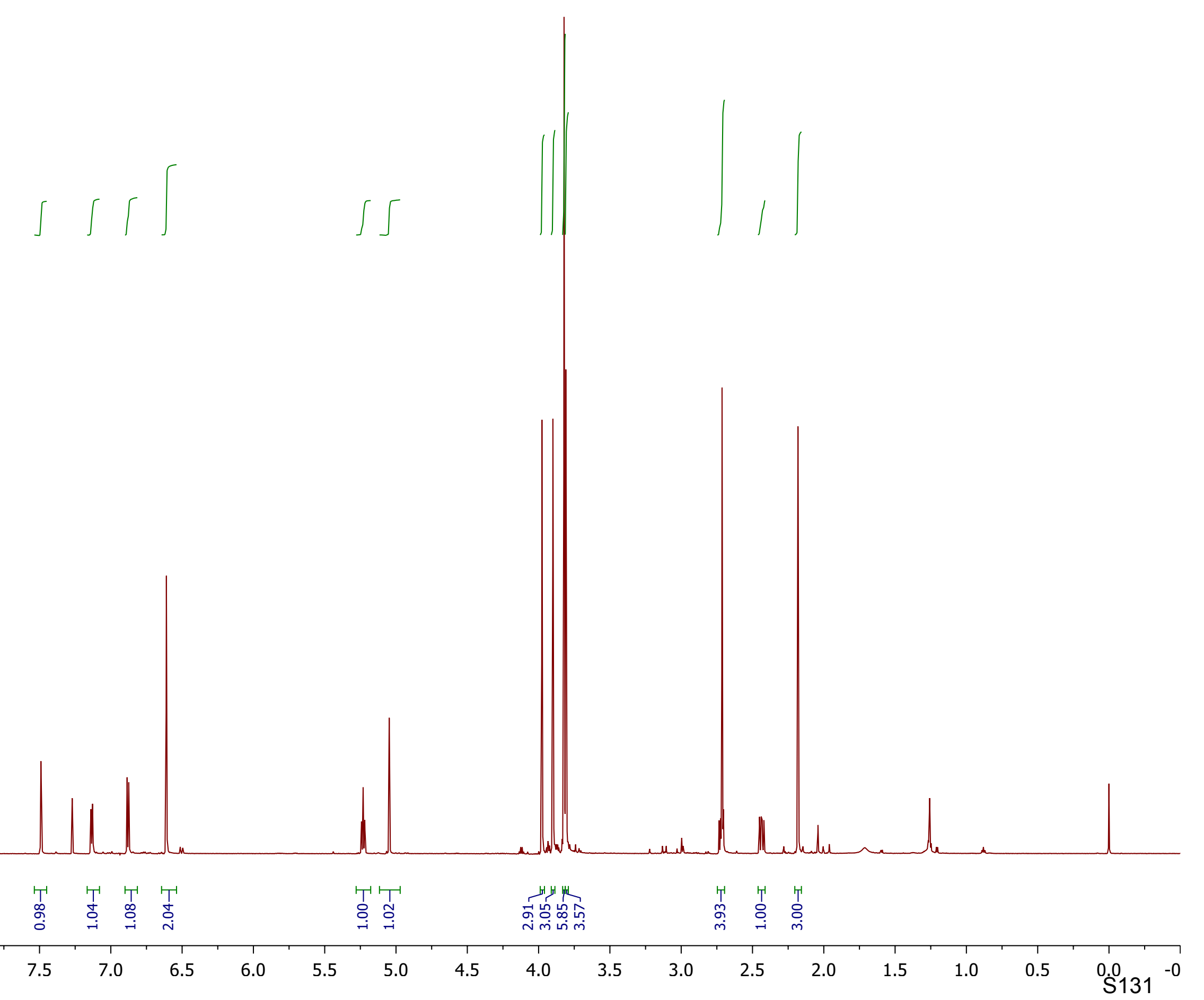




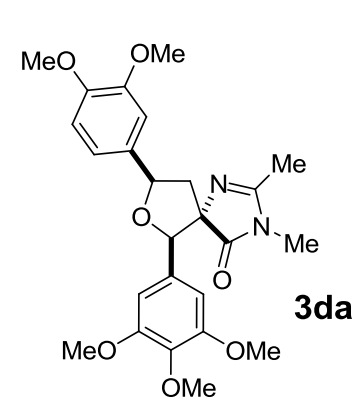

${ }^{13} \mathrm{C} \mathrm{NMR} \mathrm{(175} \mathrm{MHz),} \mathrm{CDCl}_{3}$

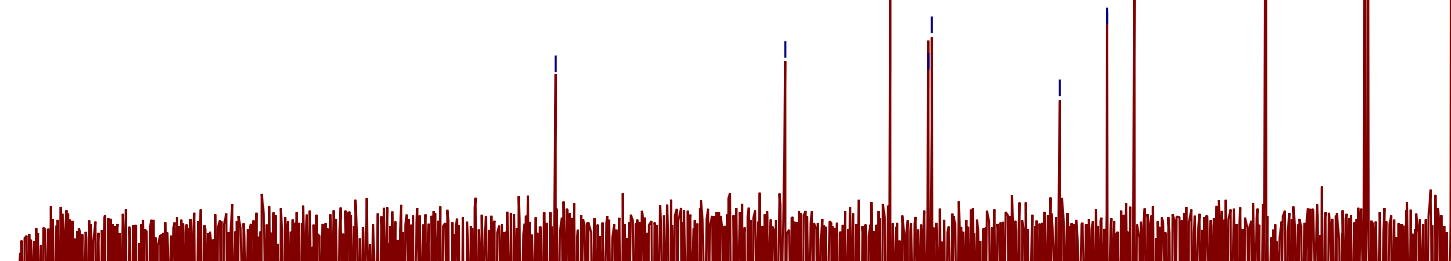




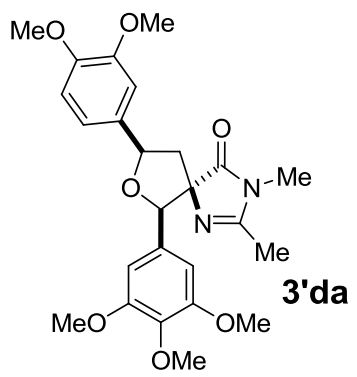

${ }^{1} \mathrm{H}$ NMR (700 MHz), $\mathrm{CDCl}_{3}$

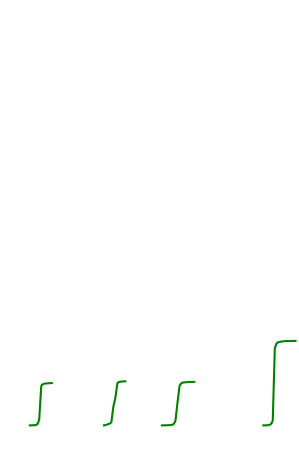

$$
\iiint \Gamma
$$
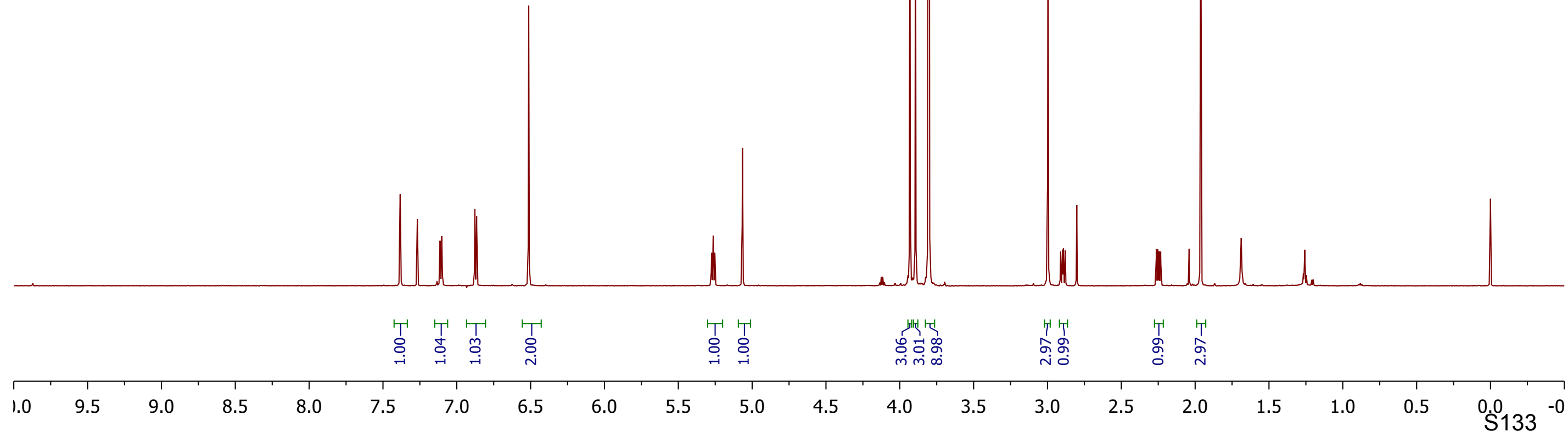


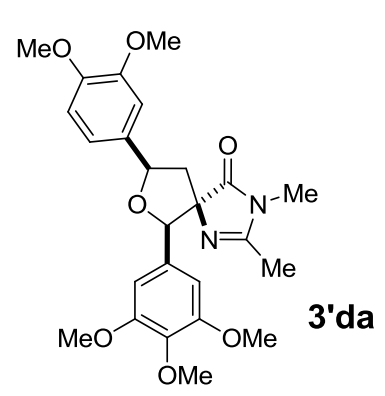

$\underset{\substack{\infty \\ \infty \\ \infty}}{\stackrel{\infty}{1}}$

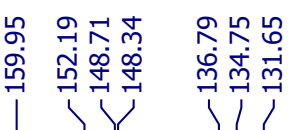

|

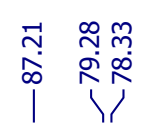

कำ

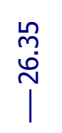

$\underset{\substack{\infty \\ \dot{1}}}{\stackrel{\infty}{\mid c}}$

${ }^{13} \mathrm{C}$ NMR (75 MHz), DMSO-d 6

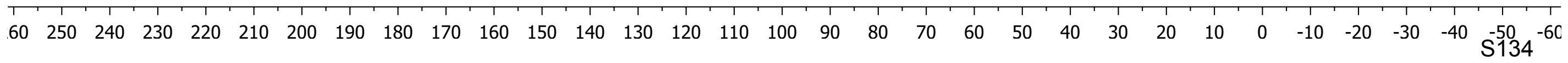




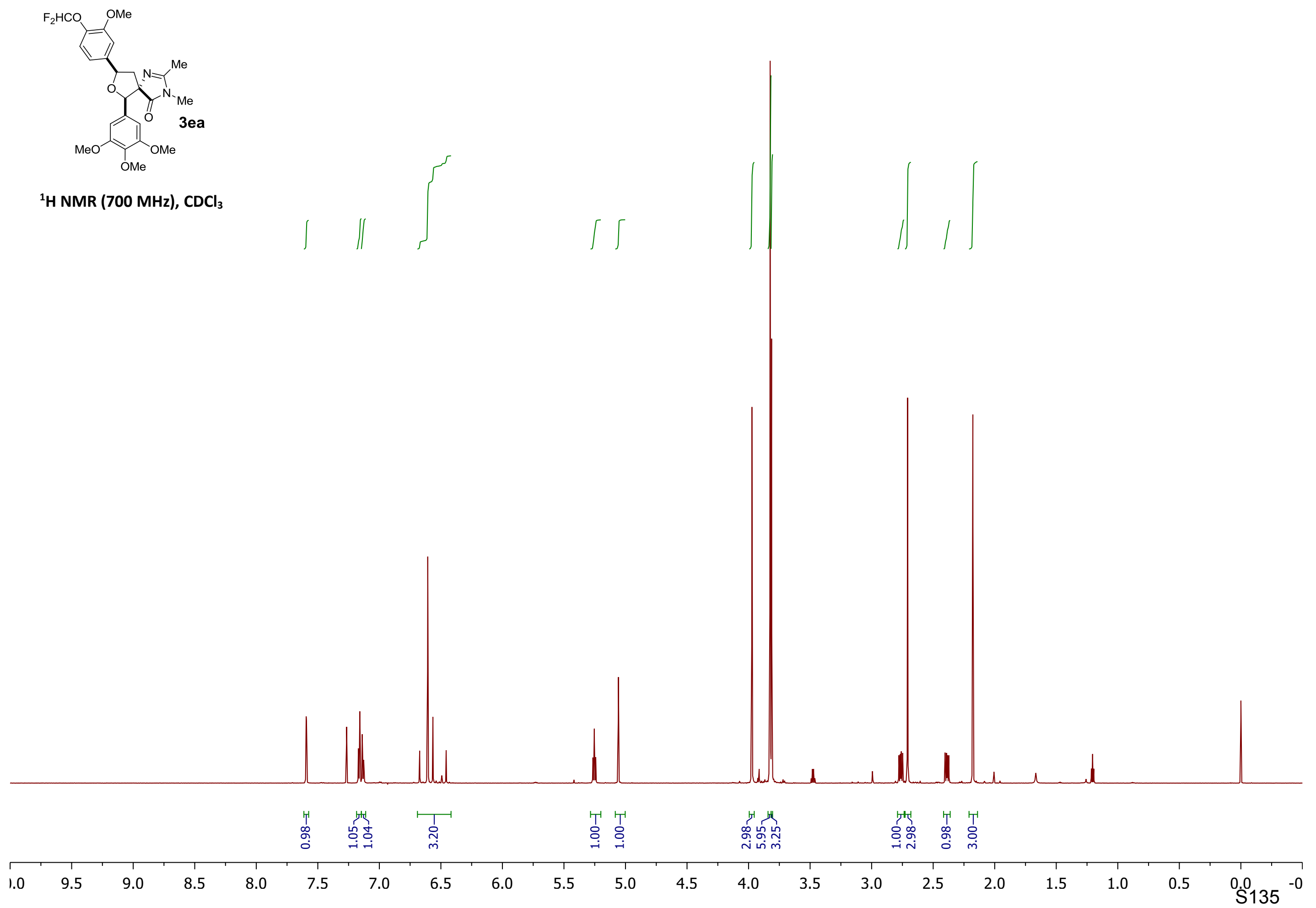




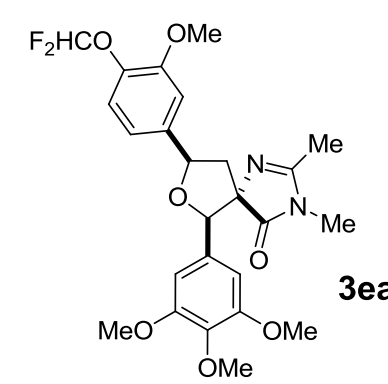

${ }^{13} \mathrm{C} \mathrm{NMR} \mathrm{(175} \mathrm{MHz),} \mathrm{CDCl}_{3}$

$\begin{array}{llllllllllllllllllllllllll}220 & 210 & 200 & 190 & 180 & 170 & 160 & 150 & 140 & 130 & 120 & 110 & 100 & 90 & 80 & 70 & 60 & 50 & 40 & 30 & 20 & 10 & 0 & -10 & 5136\end{array}$




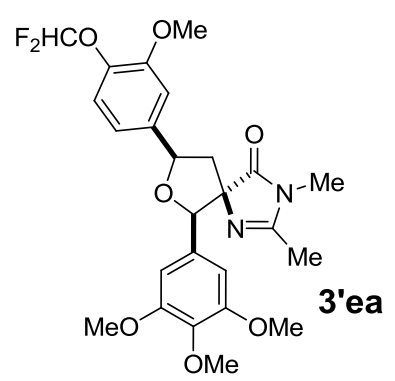

${ }^{1} \mathrm{H}$ NMR (700 MHz), $\mathrm{CDCl}_{3}$ 


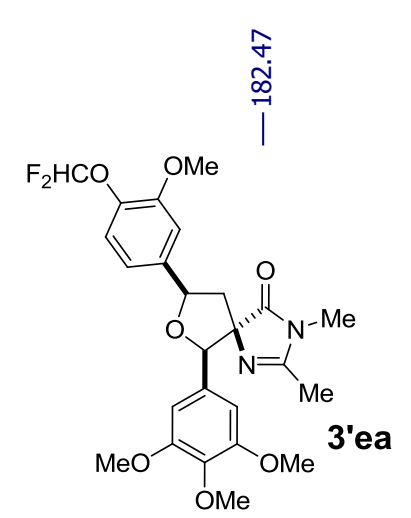

${ }^{13} \mathrm{C}$ NMR (175 MHz), $\mathrm{CDCl}_{3}$

\section{$\mathrm{EtOH}$}

(9 w\%)

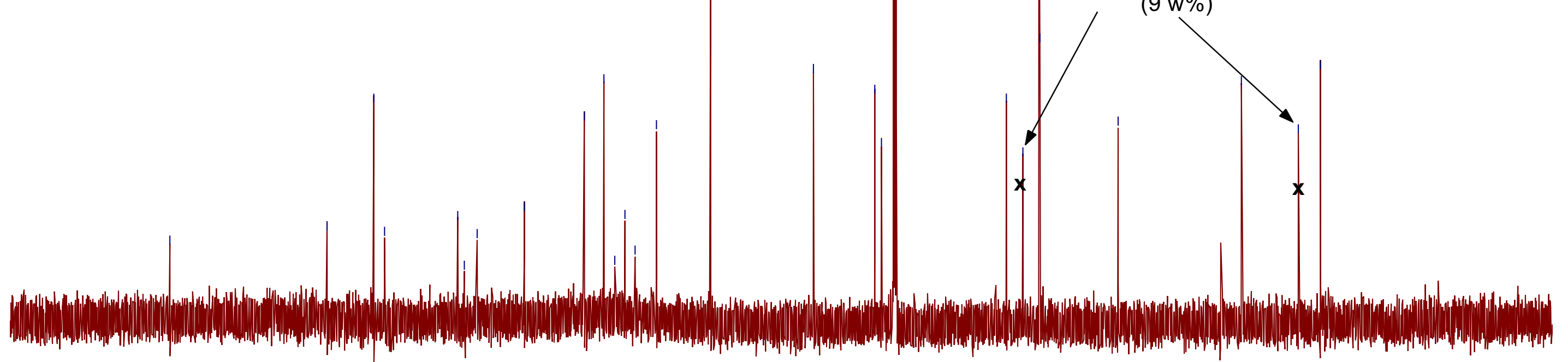




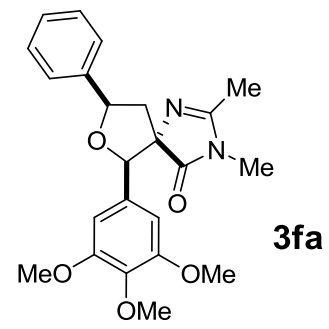

${ }^{1} \mathrm{H}$ NMR (700 MHz), $\mathrm{CDCl}_{3}$
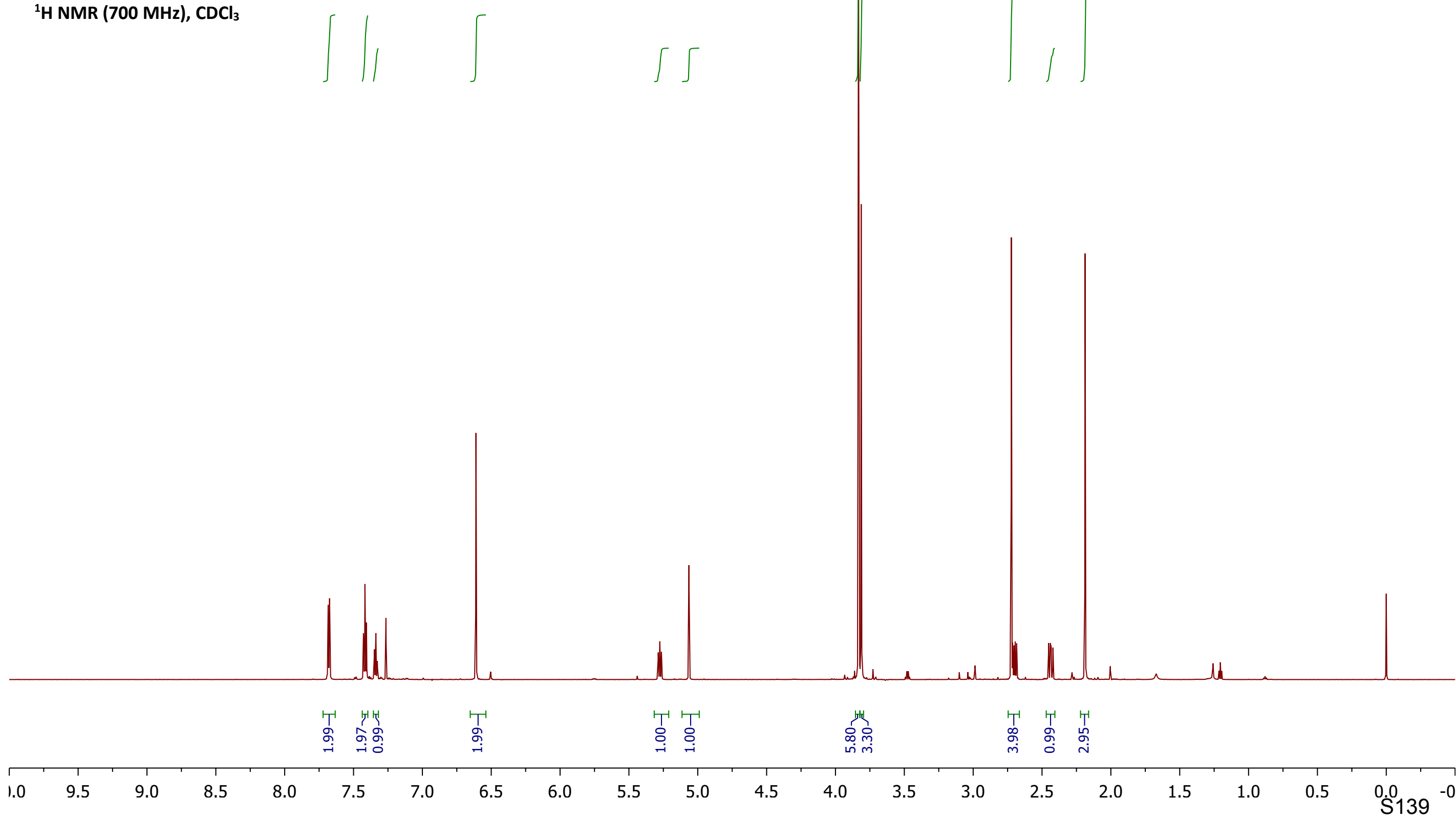


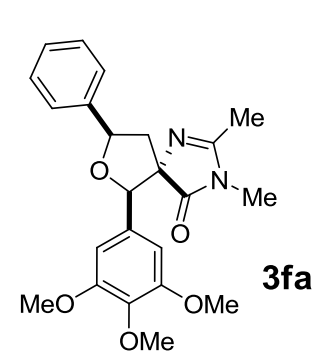

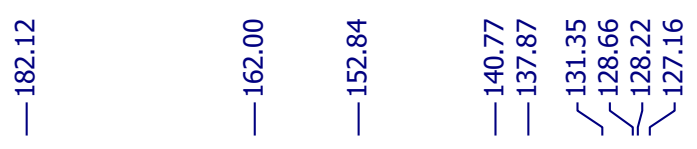

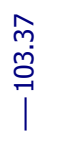

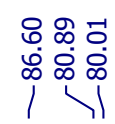

$\begin{array}{ll}\text { ลิ } & \text { 옹 } \\ 0 & 0 \\ 1 & 1\end{array}$

$\stackrel{f}{\mathfrak{J}}$

总

${ }^{13} \mathrm{C} \mathrm{NMR} \mathrm{(175} \mathrm{MHz),} \mathrm{CDCl}_{3}$
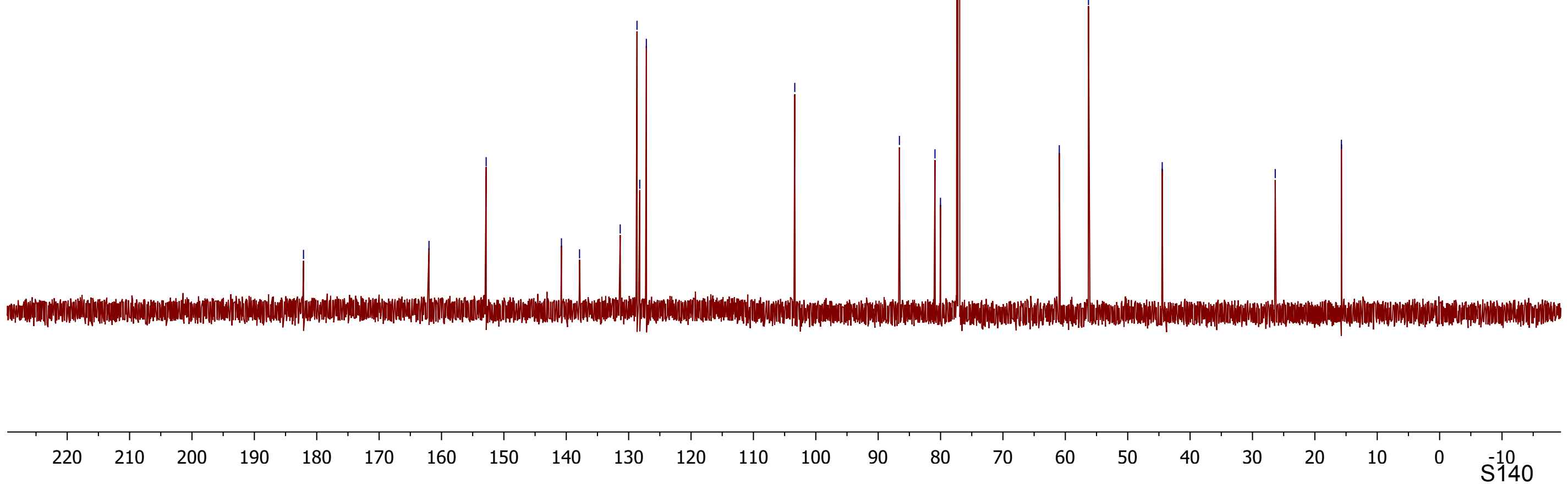


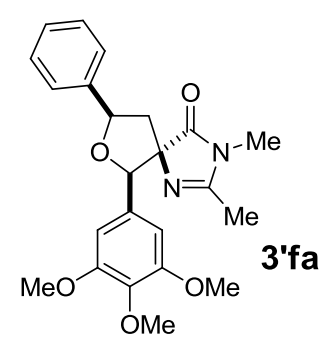

${ }^{1} \mathrm{H}$ NMR (700 MHz), $\mathrm{CDCl}_{3}$

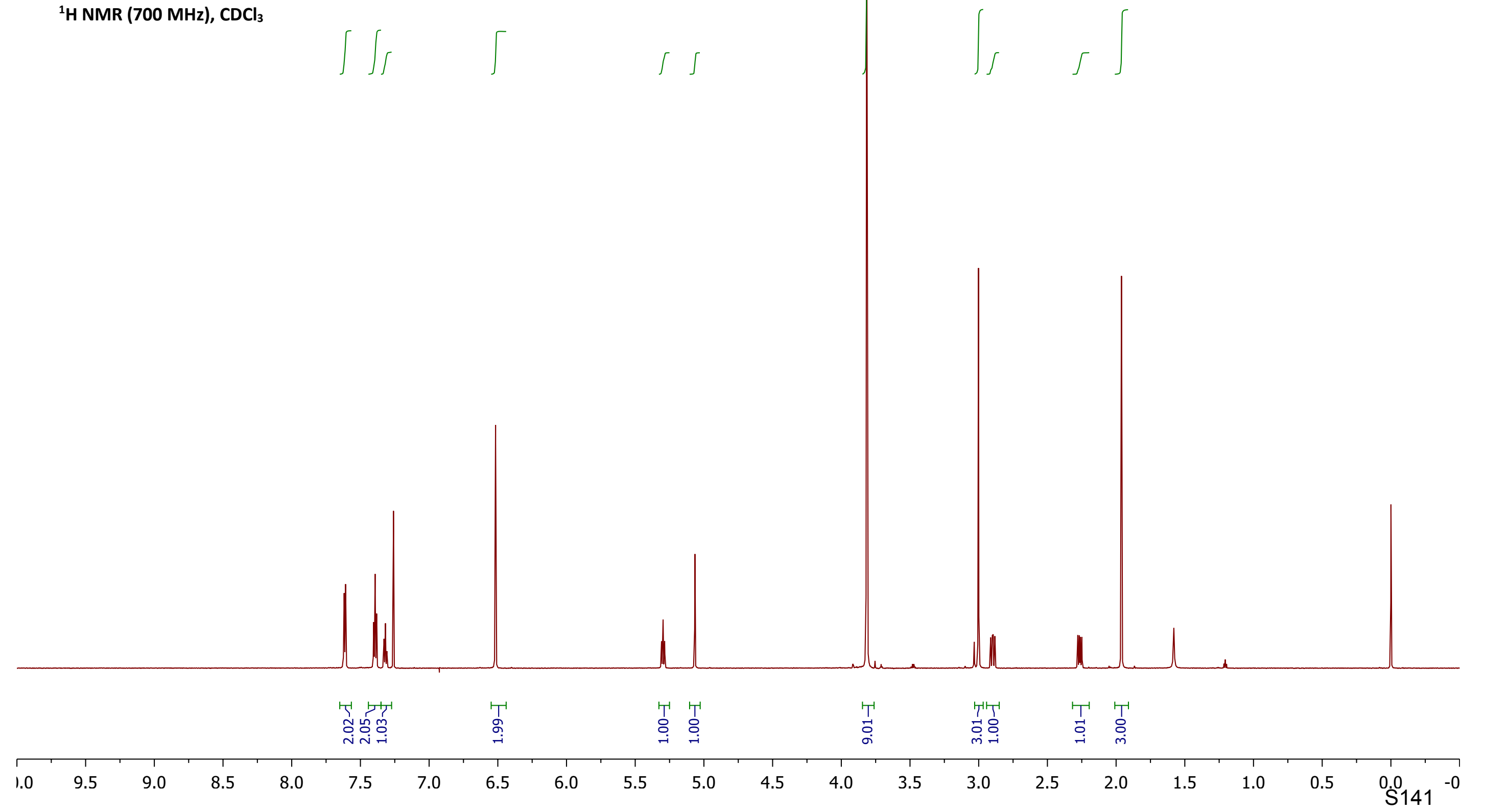





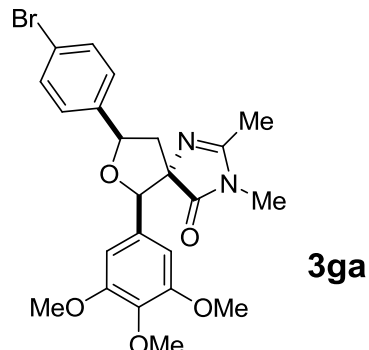

${ }^{1} \mathrm{H}$ NMR $(700 \mathrm{MHz}), \mathrm{CDCl}_{3}$

Contains $12 \%$ of impurity

(presumably diastereomer)

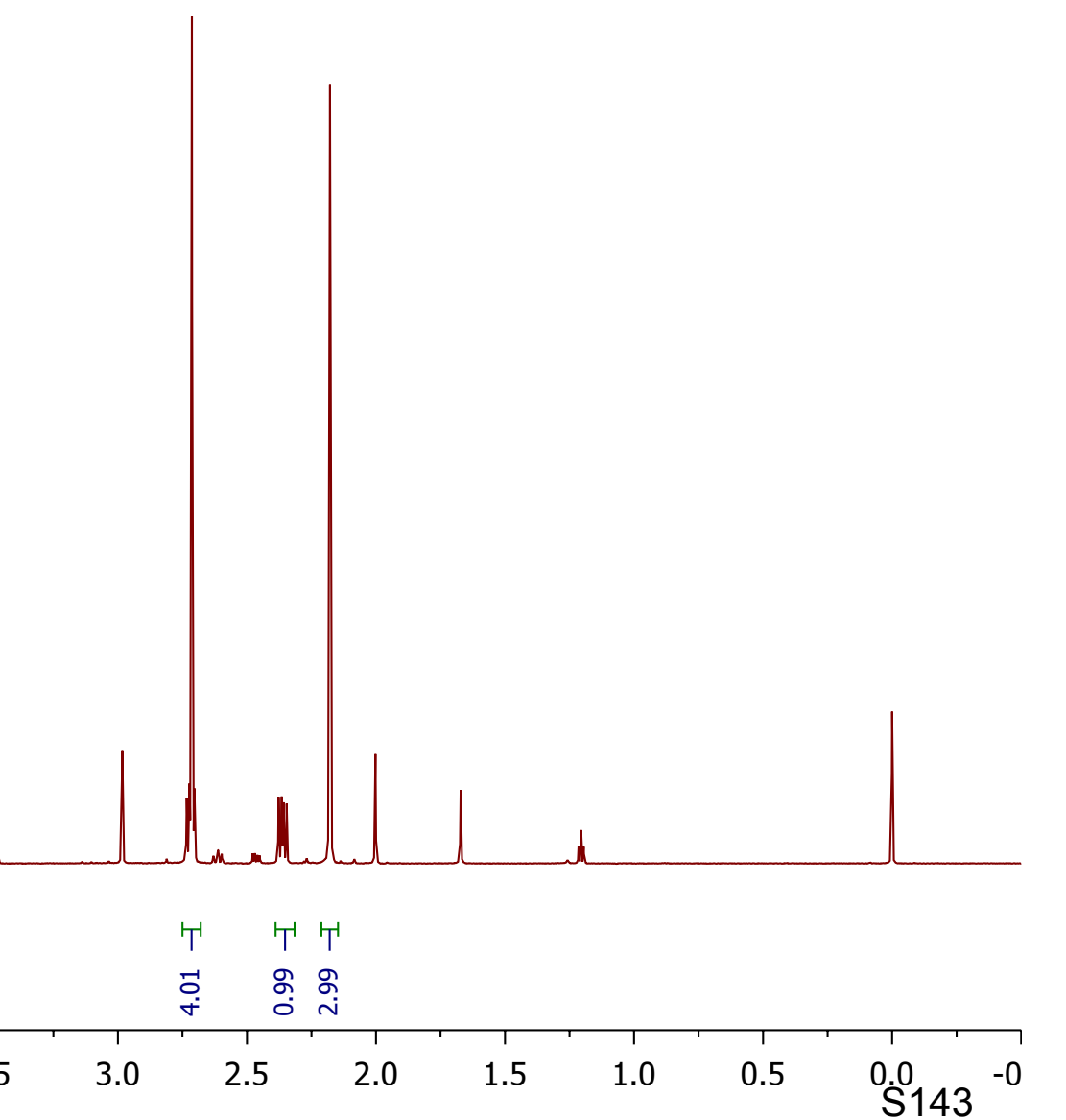




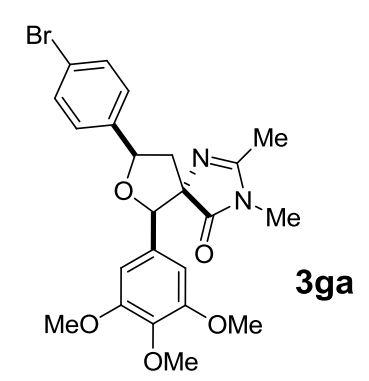

${ }^{13} \mathrm{C}$ NMR (175 MHz), $\mathrm{CDCl}_{3}$

Contains $12 \%$ of impurity

(presumably diastereomer) 


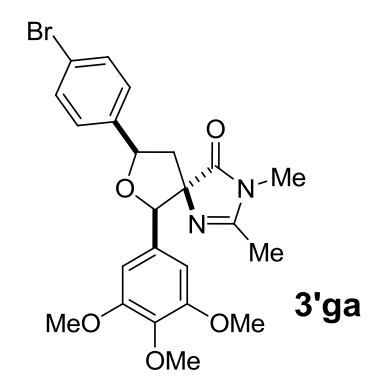

${ }^{1} \mathrm{H}$ NMR (700 MHz), $\mathrm{CDCl}_{3}$

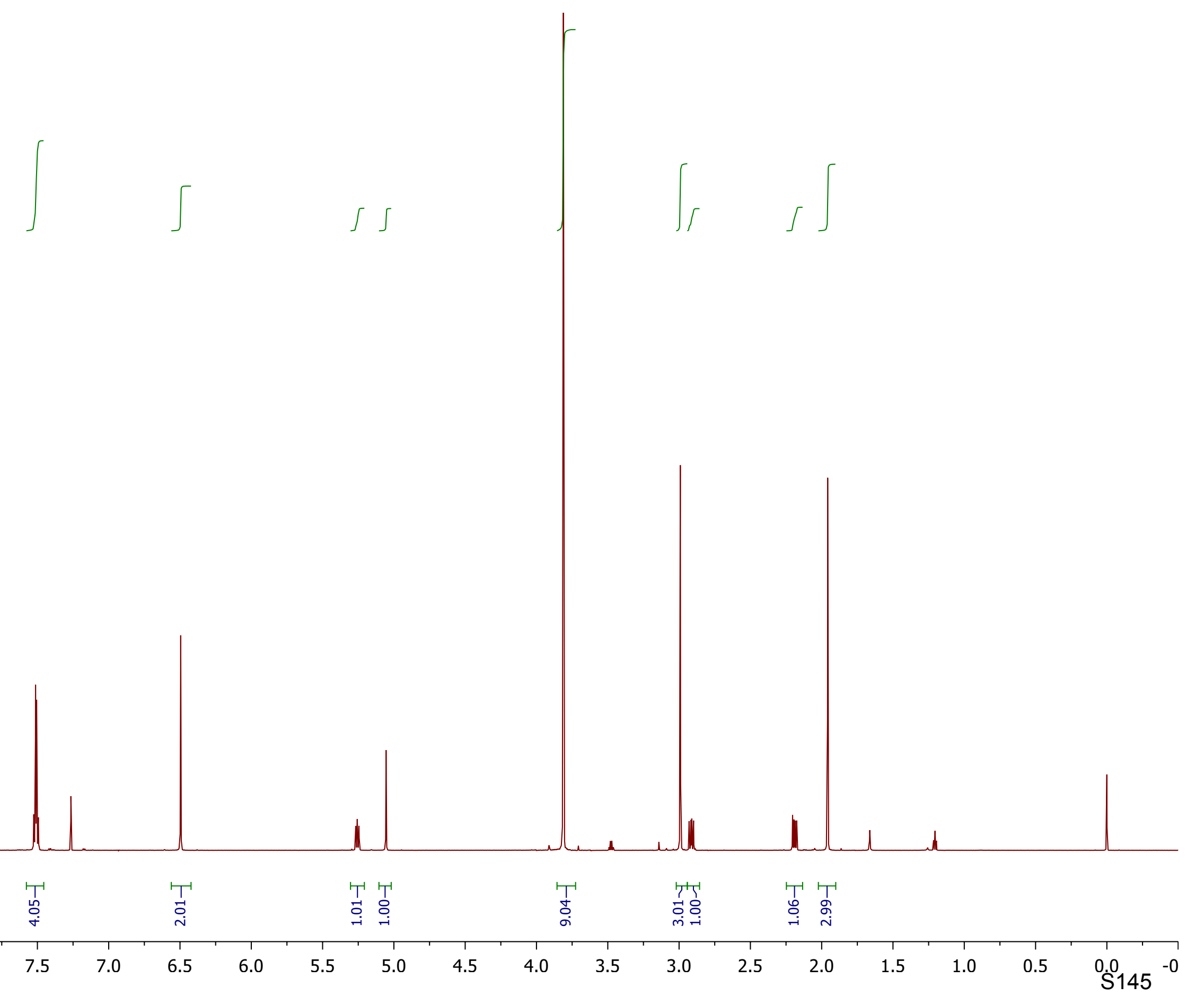




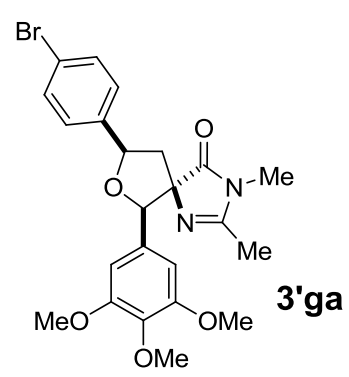

|

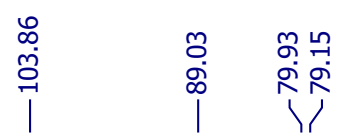

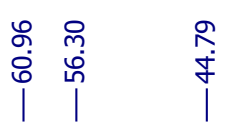

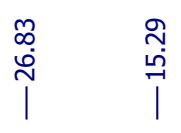

${ }^{13} \mathrm{C} \mathrm{NMR} \mathrm{(175} \mathrm{MHz),} \mathrm{CDCl}_{3}$
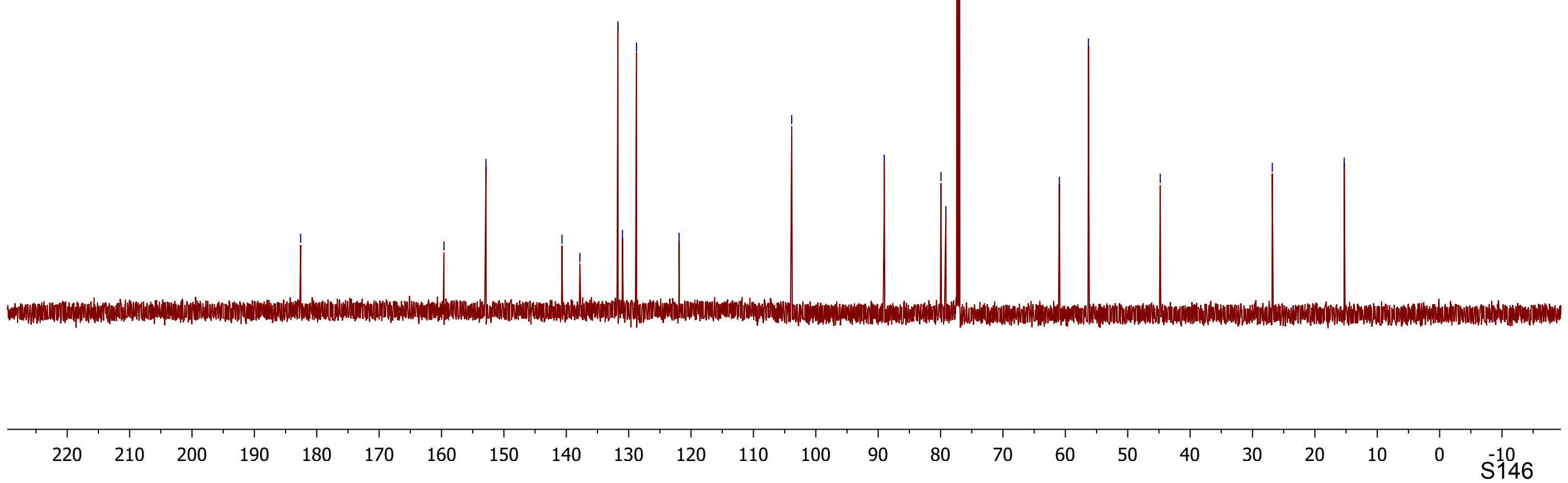


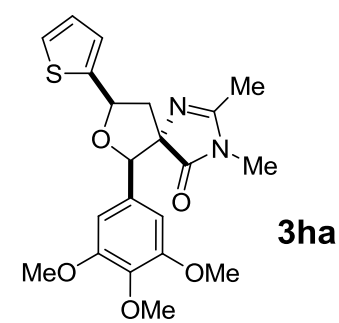

${ }^{1} \mathrm{H}$ NMR (700 MHz), $\mathrm{CDCl}_{3}$

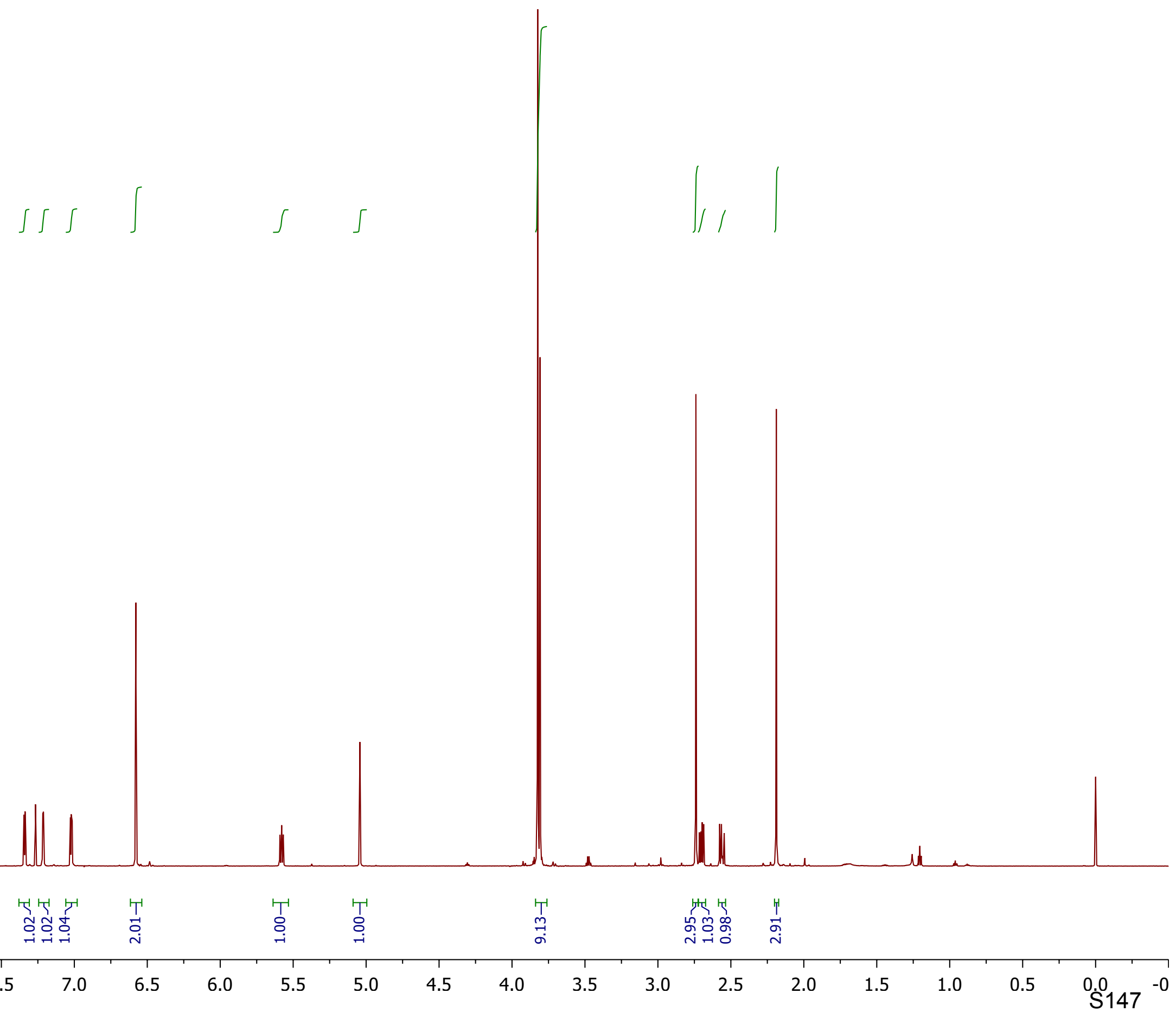




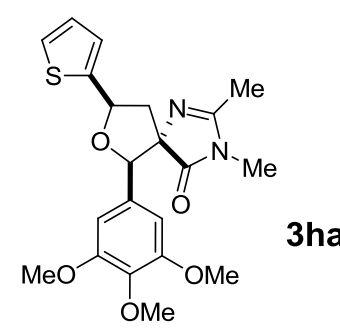

\section{${ }^{13} \mathrm{C}$ NMR (175 MHz), $\mathrm{CDCl}_{3}$}

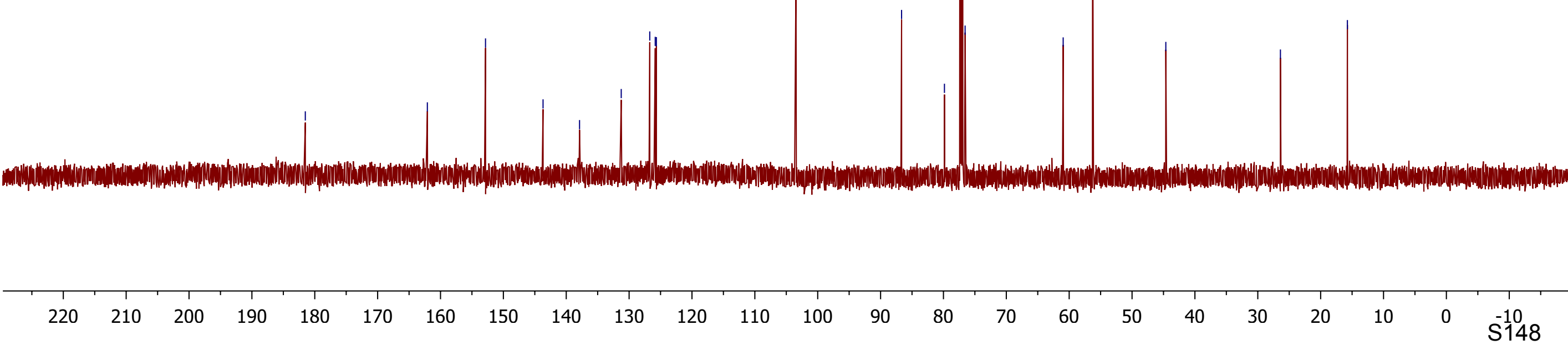




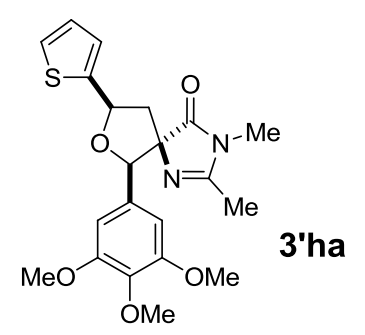

${ }^{1} \mathrm{H}$ NMR (700 MHz), $\mathrm{CDCl}_{3}$

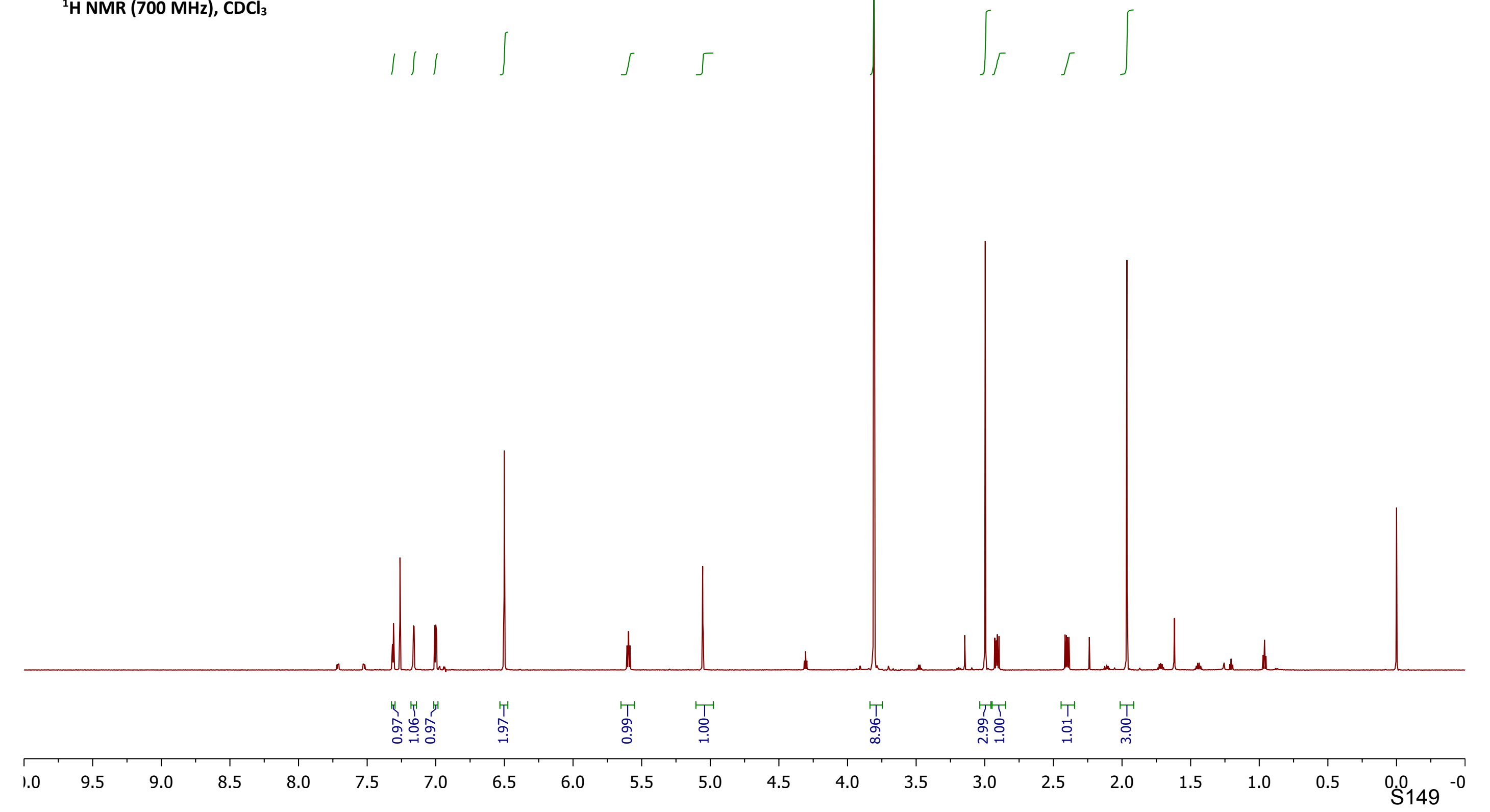




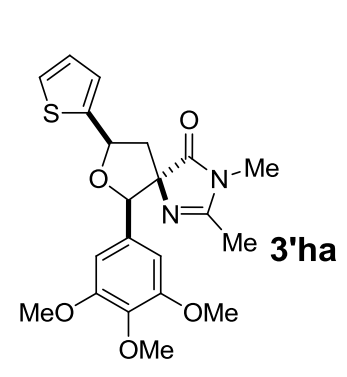

${ }^{13} \mathrm{C}$ NMR (75 MHz), $\mathrm{CDCl}_{3}$

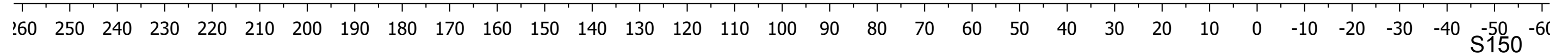





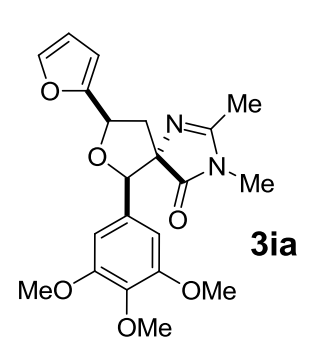

${ }^{13} \mathrm{C}$ NMR (175 MHz), $\mathrm{CDCl}_{3}$ i

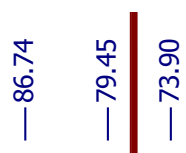

옹ํำ

$\stackrel{\text { fे }}{\text { f }}$

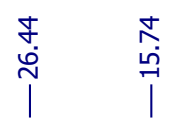

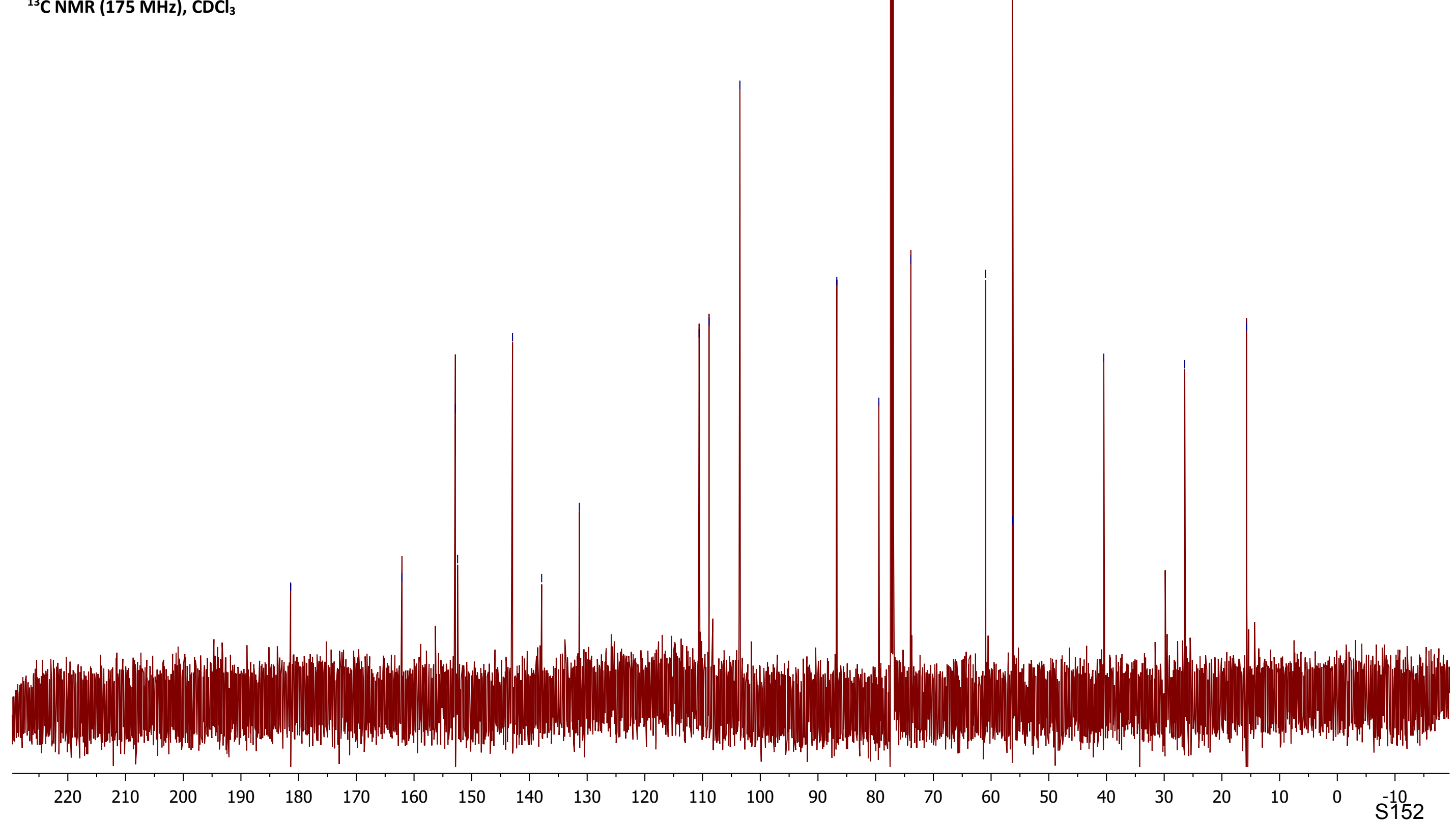




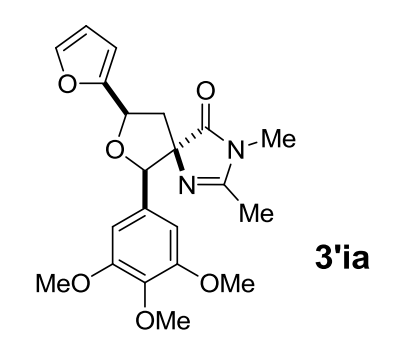

${ }^{1} \mathrm{H}$ NMR (700 MHz), $\mathrm{CDCl}_{3}$

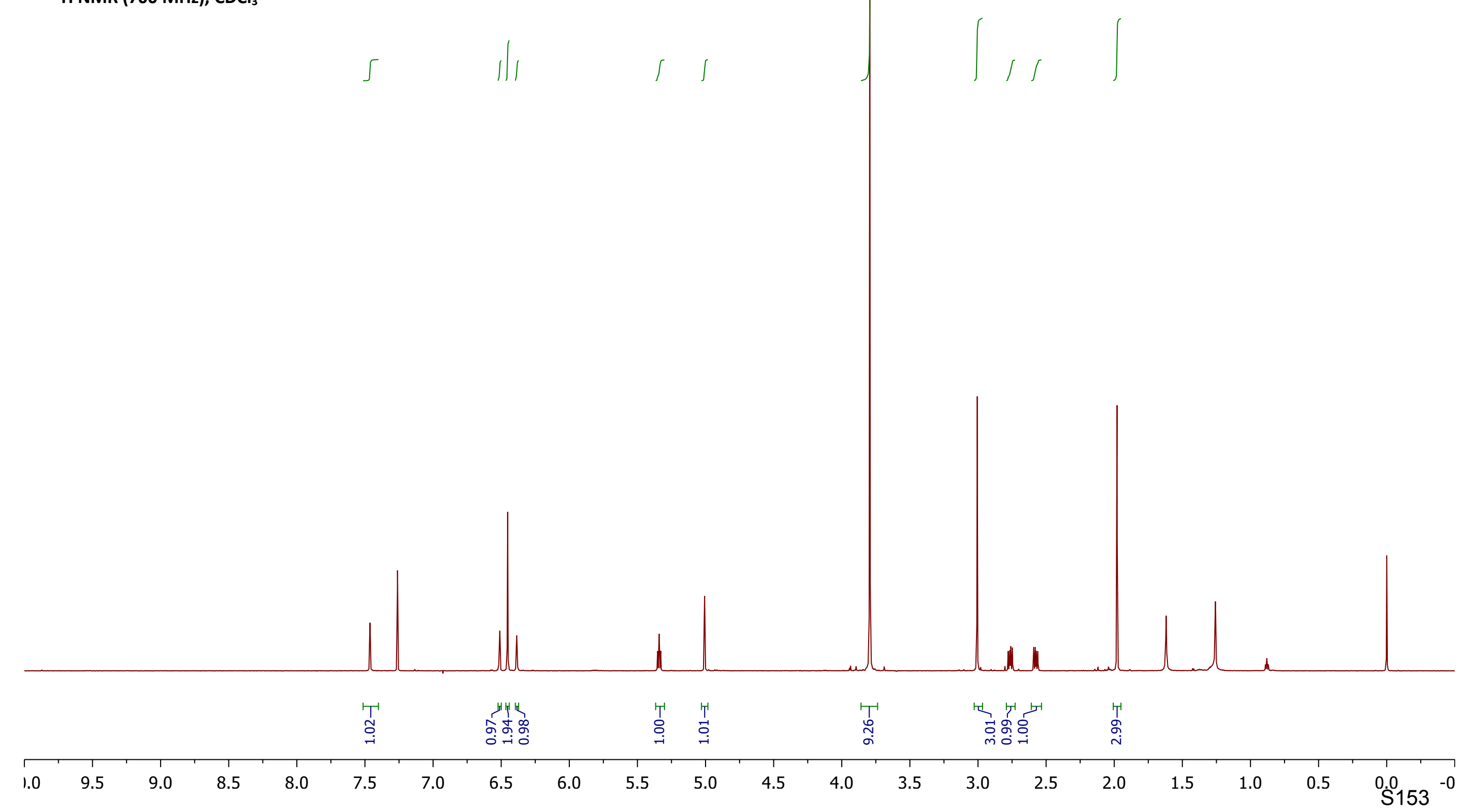




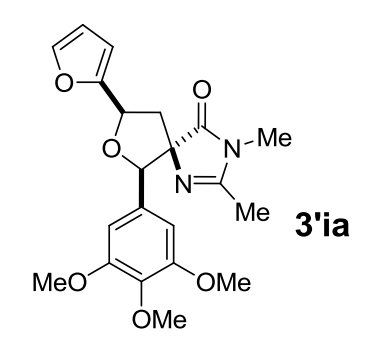

\section{${ }^{13} \mathrm{C} \mathrm{NMR}$ (75 MHz), $\mathrm{CDCl}_{3}$}




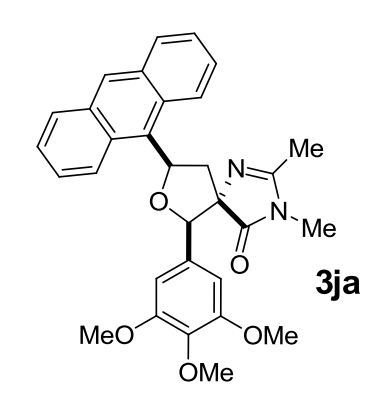

${ }^{1} \mathrm{H}$ NMR (700 MHz), $\mathrm{CDCl}_{3}$
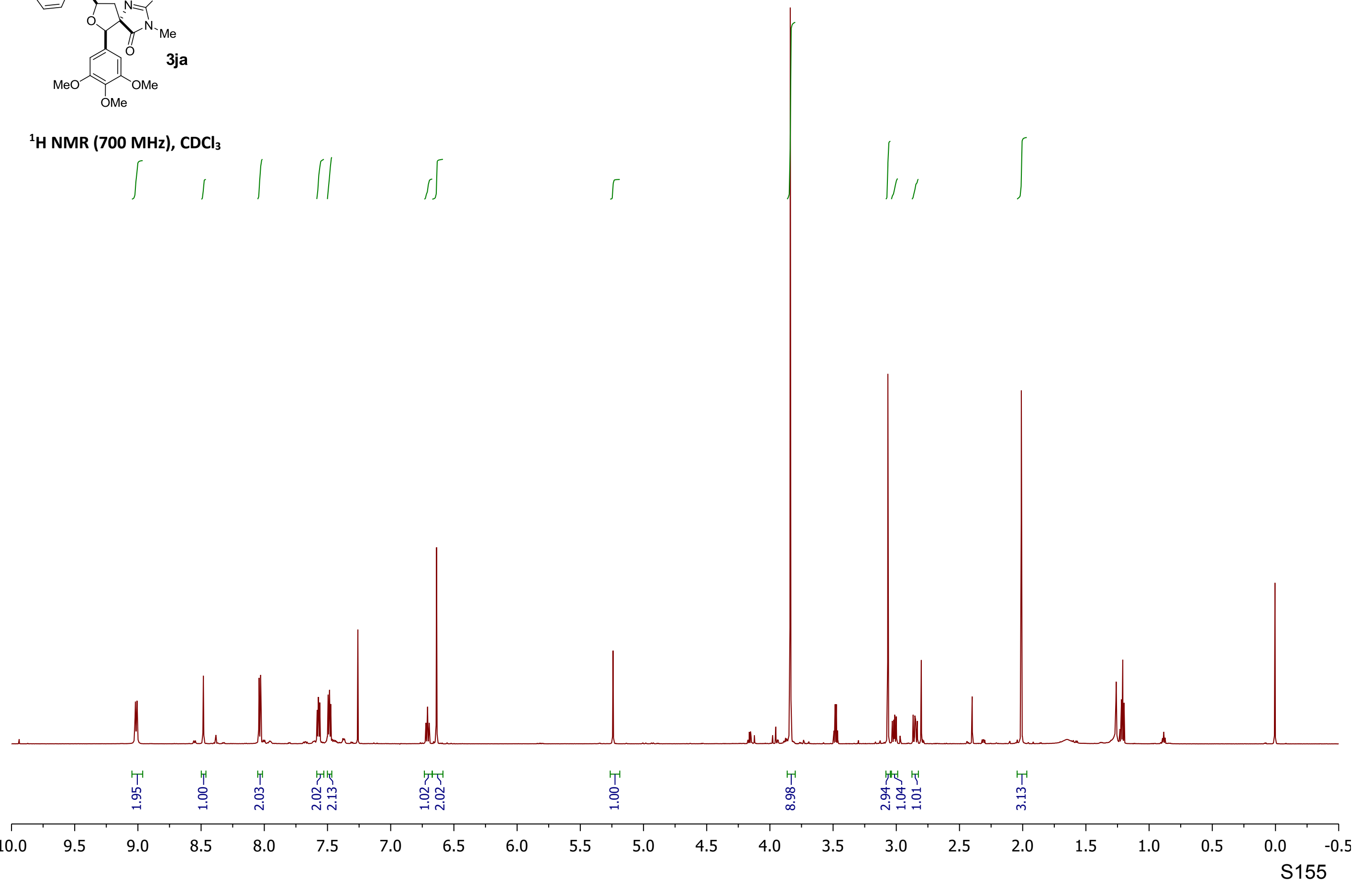


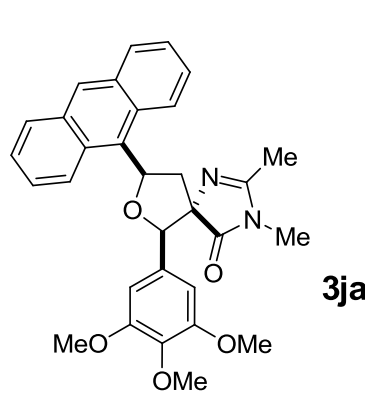

${ }^{13} \mathrm{C}$ NMR (175 MHz), $\mathrm{CDCl}_{3}$
|

|

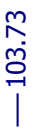

$\dot{0}$
$\stackrel{\infty}{\infty}$
1

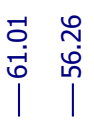

$\stackrel{\text { ஜூ }}{\text { ஜ் }}$

กู
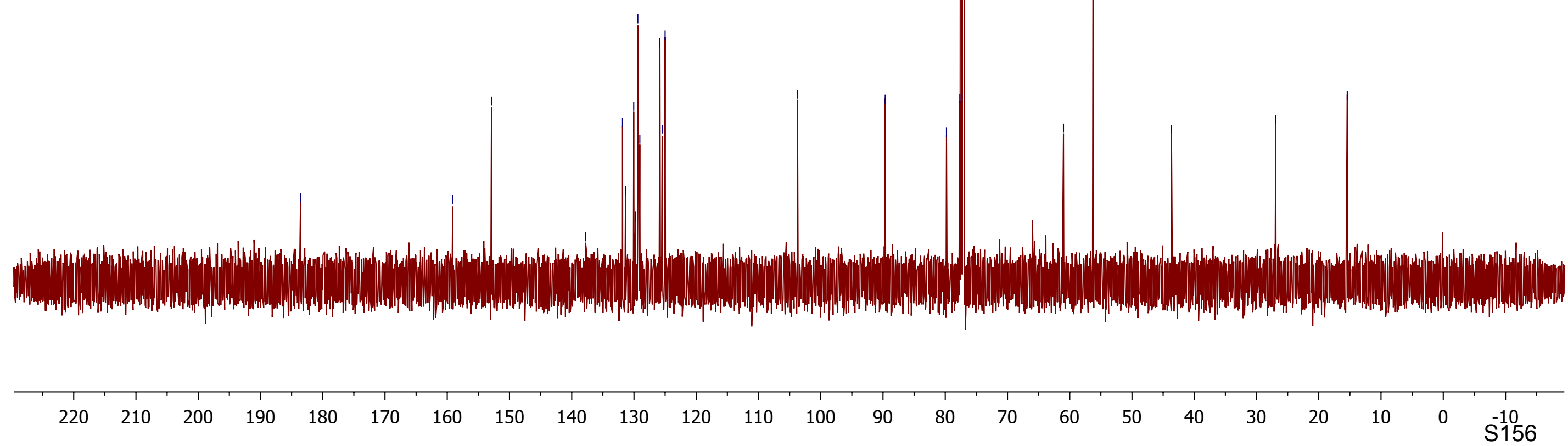
HSQC (700 MHz), $\mathrm{CDCl}_{3}$

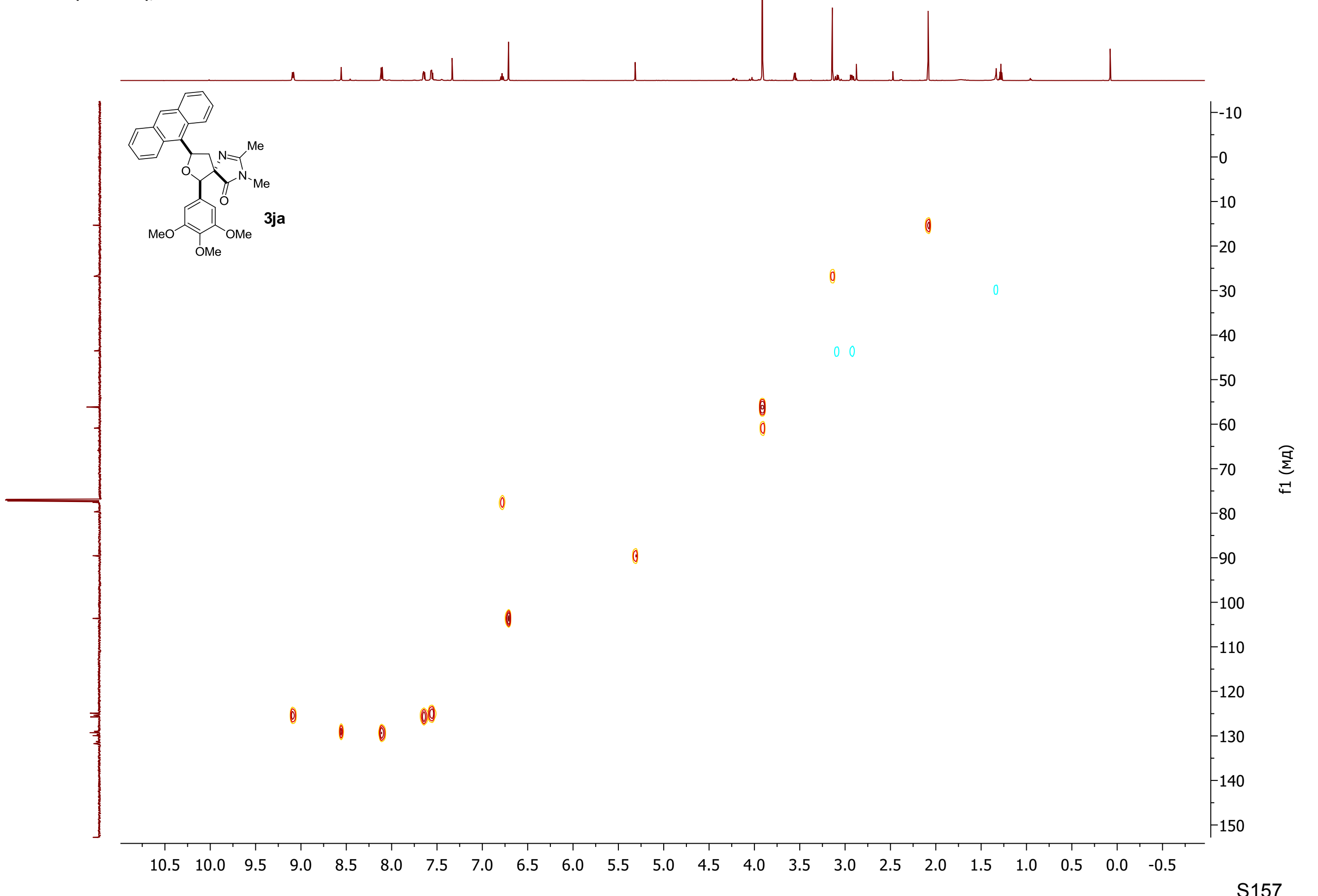


NOESY (700 MHZ), $\mathrm{CDCl}_{3}$

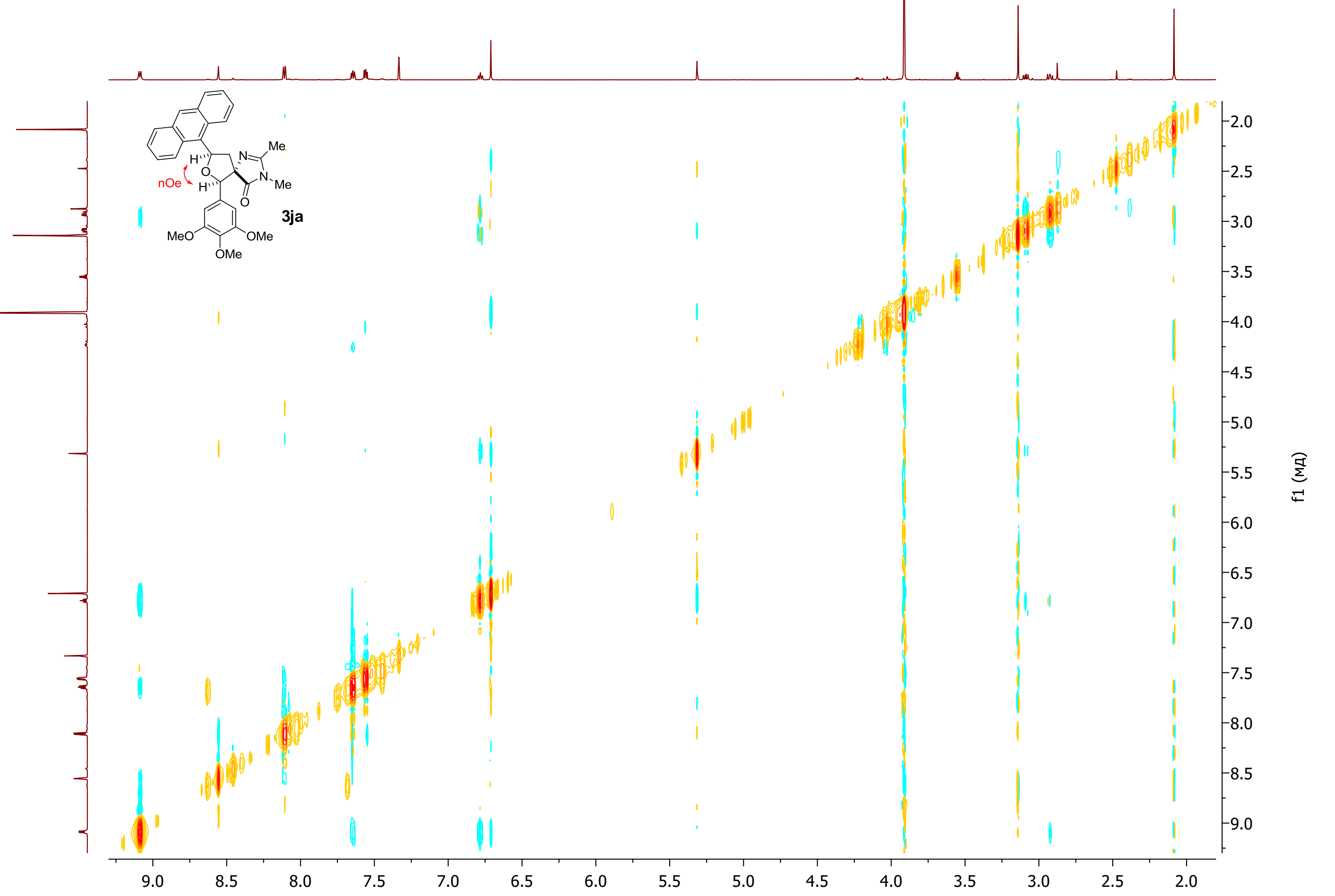




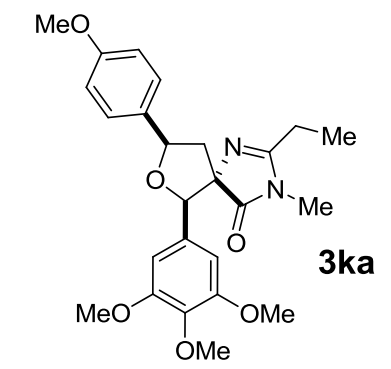

${ }^{1} \mathrm{H}$ NMR (700 MHz), $\mathrm{CDCl}_{3}$ 


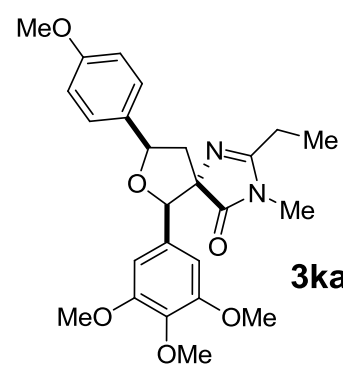

${ }^{13} \mathrm{C}$ NMR (175 MHz), $\mathrm{CDCl}_{3}$ 


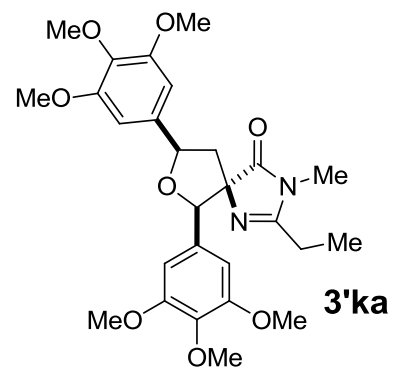

${ }^{1} \mathrm{H}$ NMR (700 MHz), $\mathrm{CDCl}_{3}$
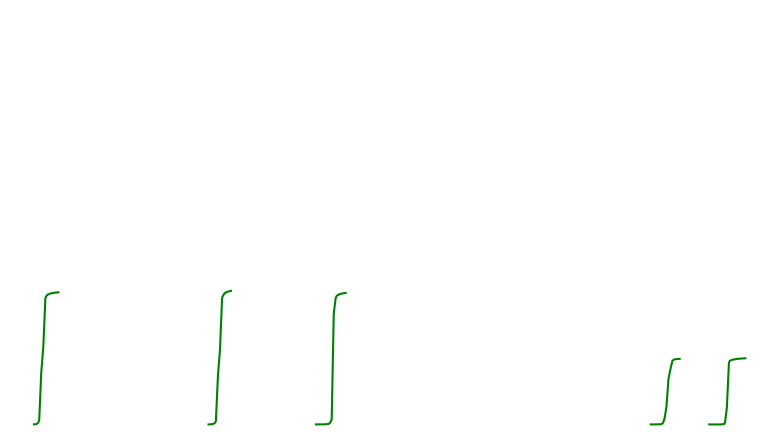

$\int$
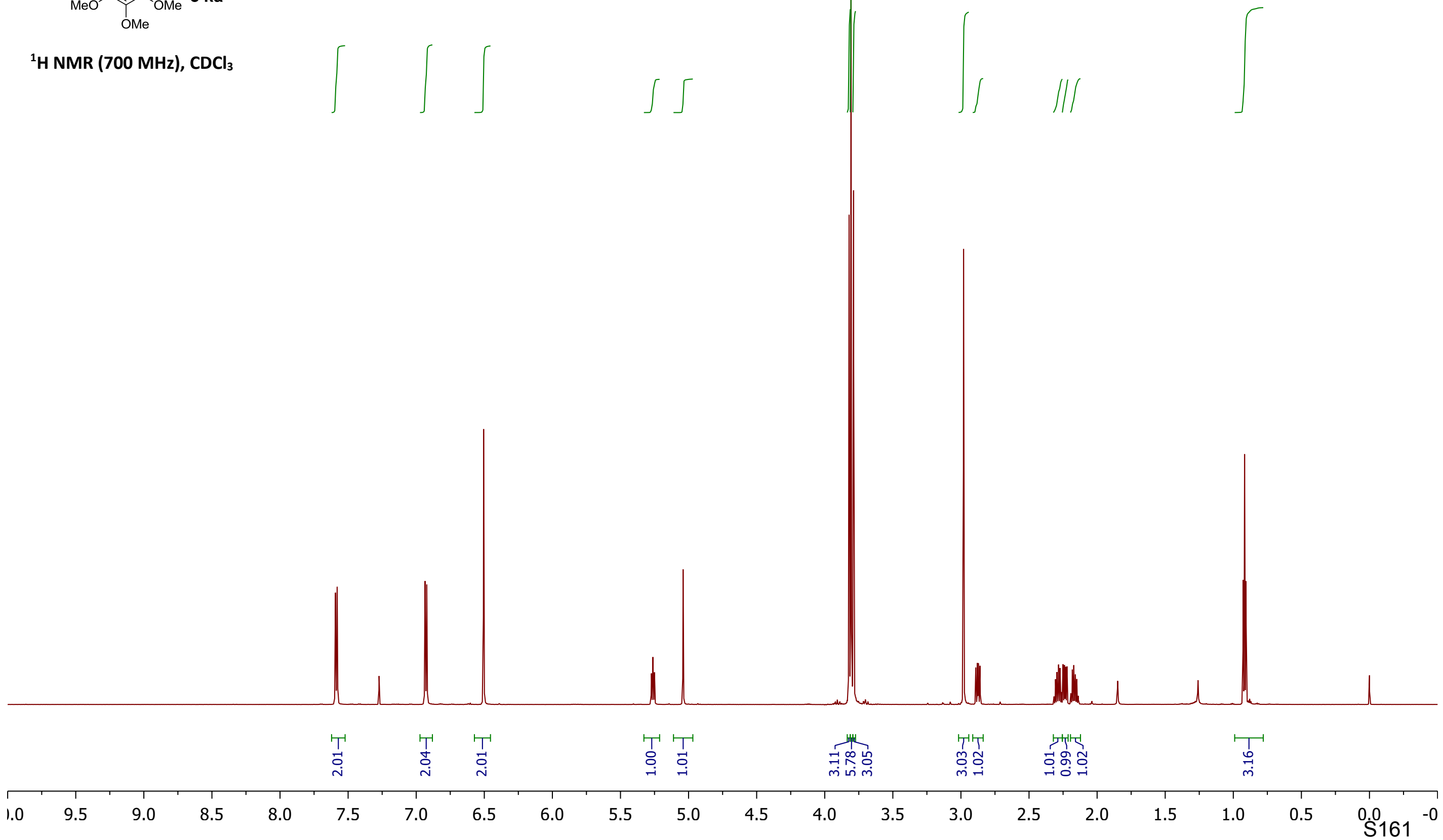


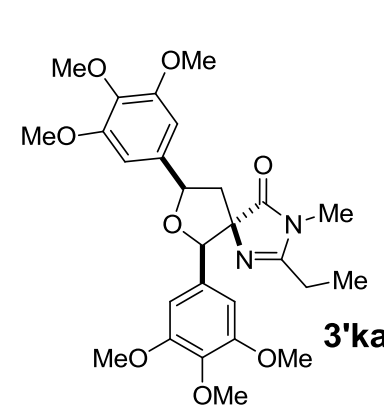

${ }^{13} \mathrm{C}$ NMR (75 MHz), $\mathrm{CDCl}_{3}$

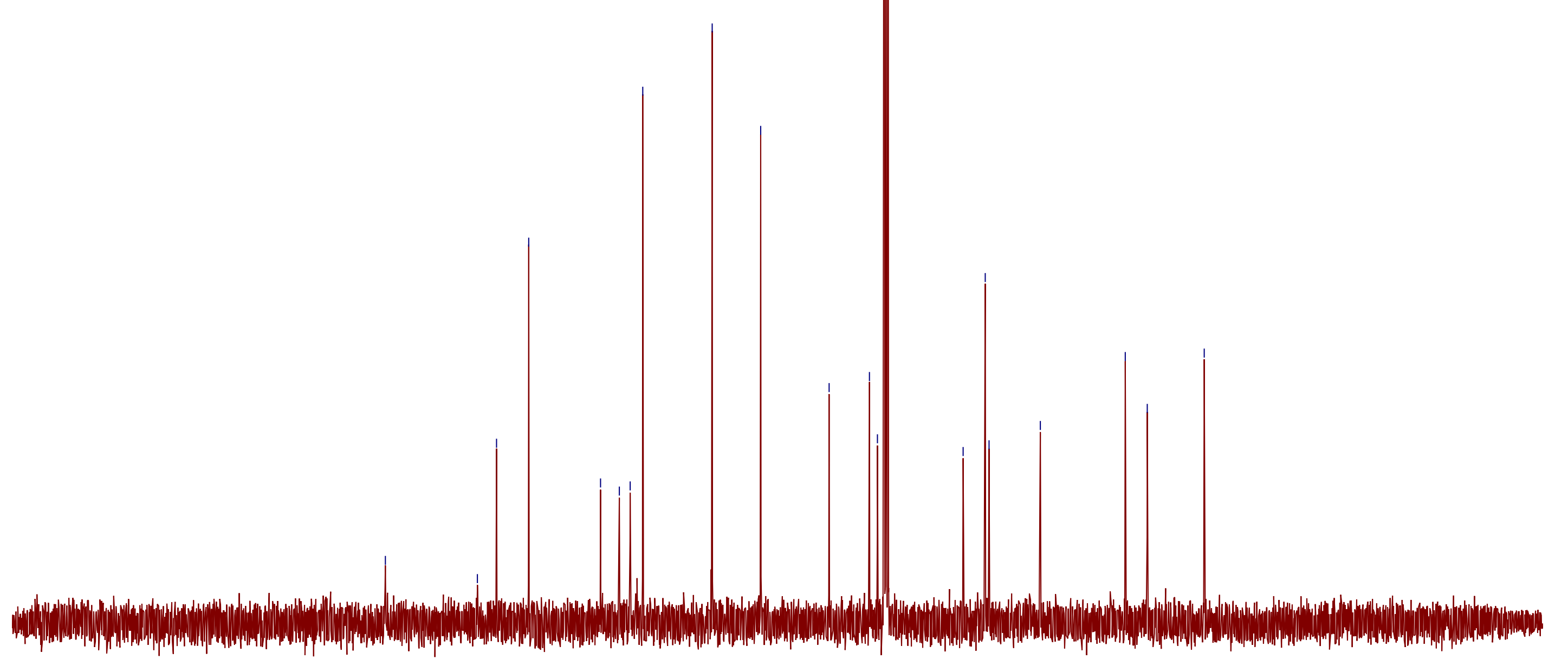

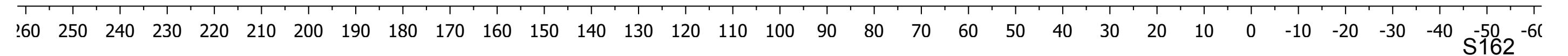



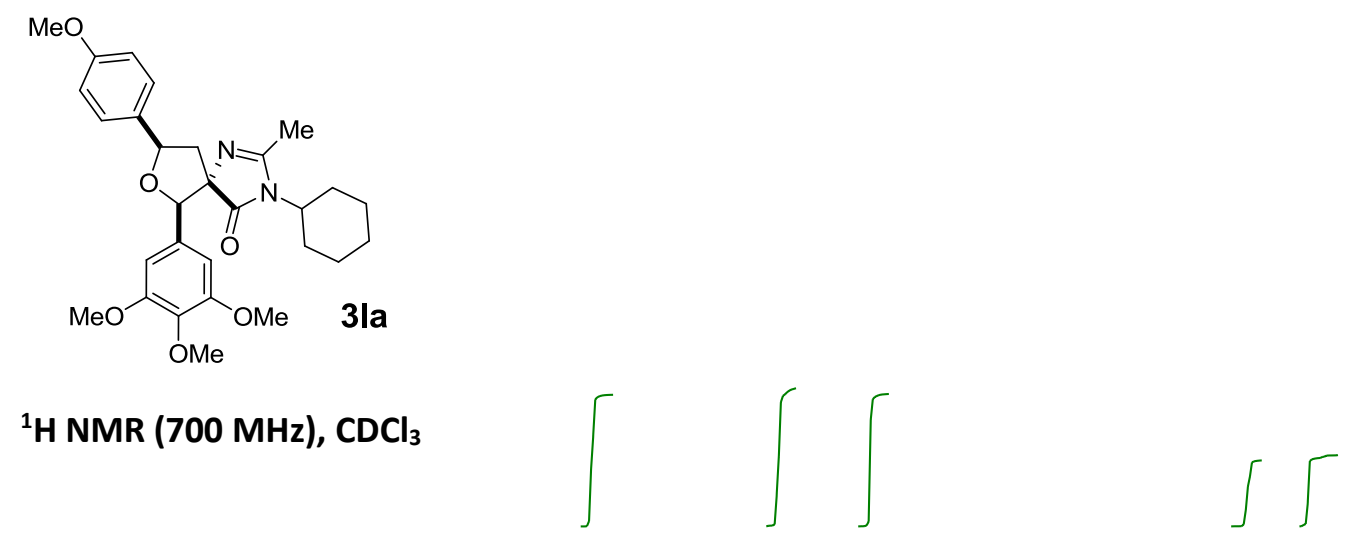

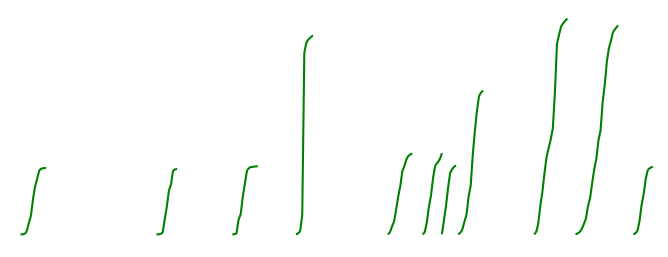

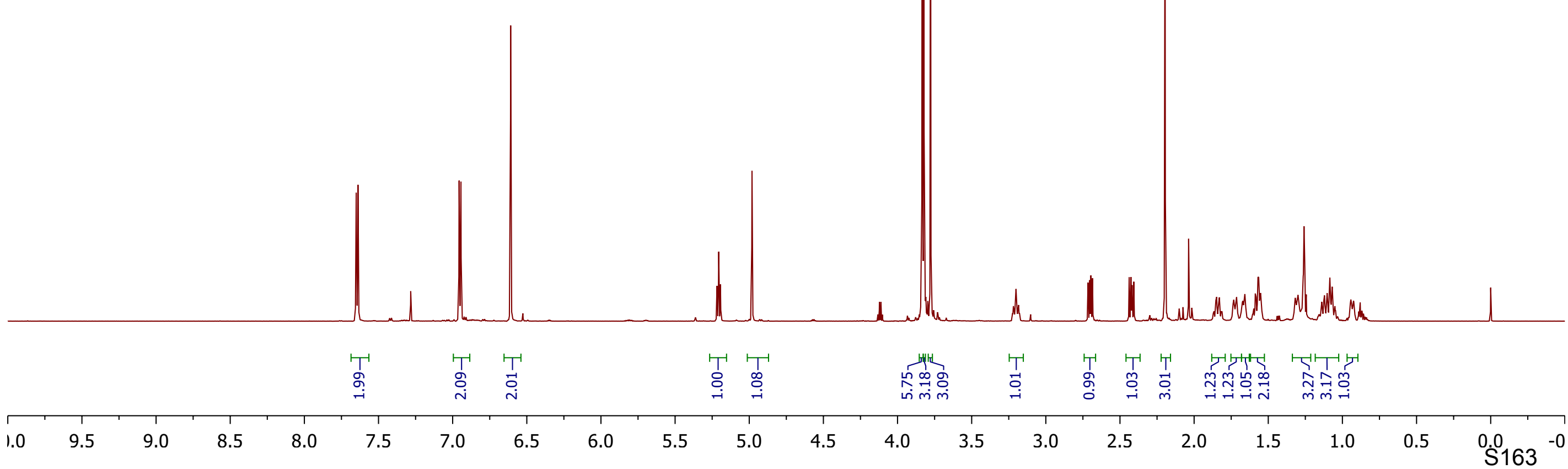




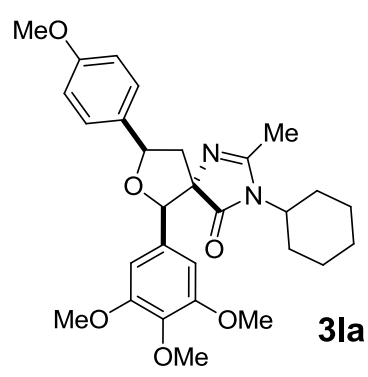

${ }^{13} \mathrm{C}$ NMR (175 MHz), $\mathrm{CDCl}_{3}$

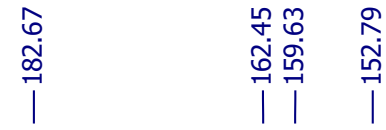

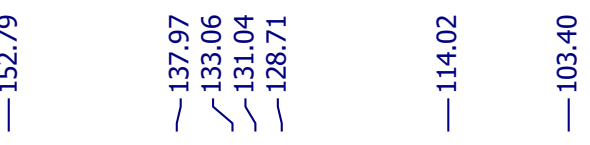

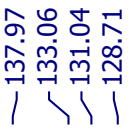

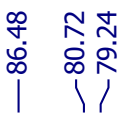

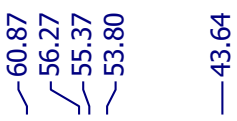

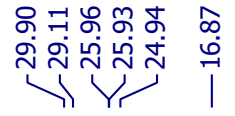
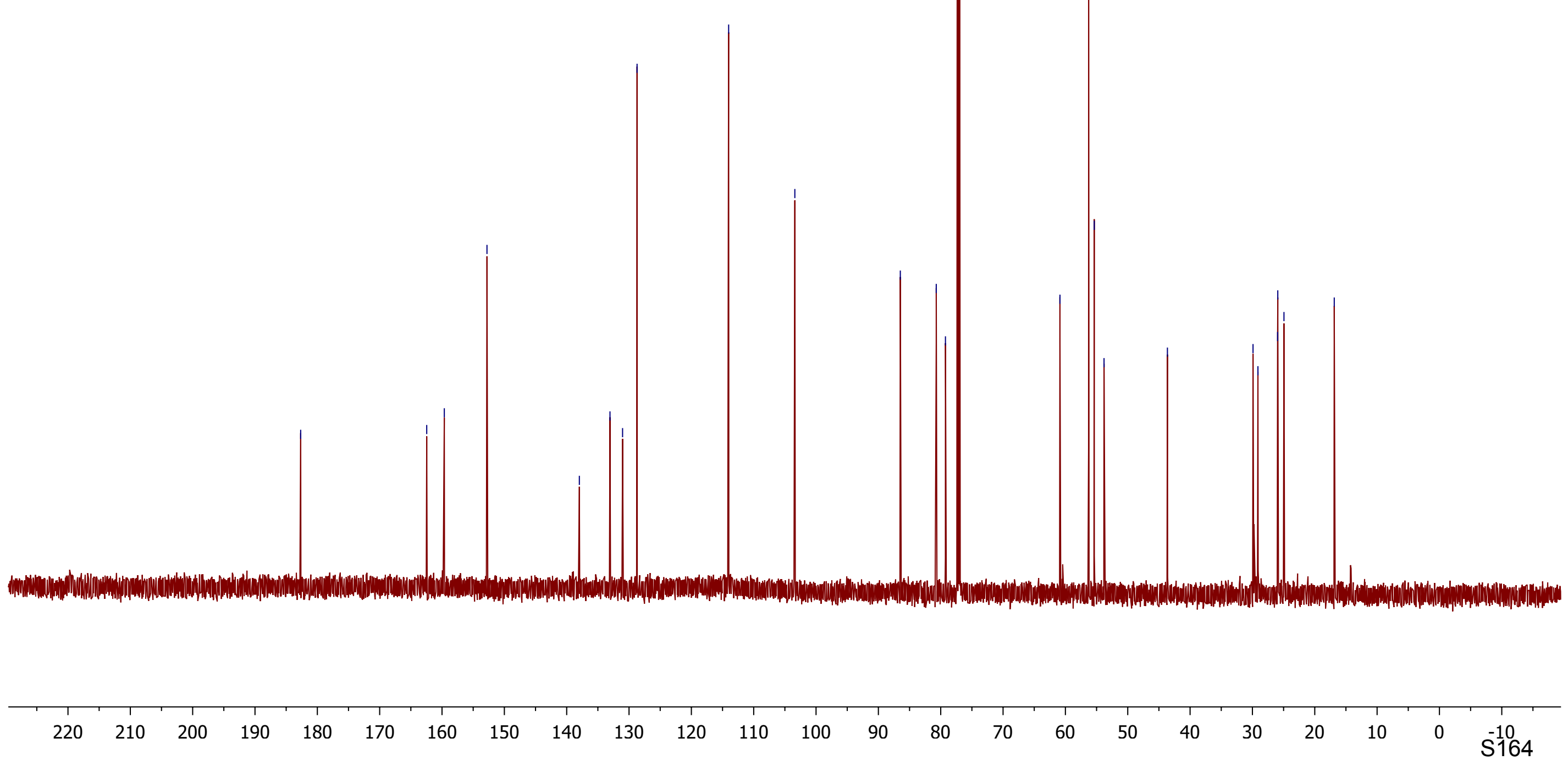


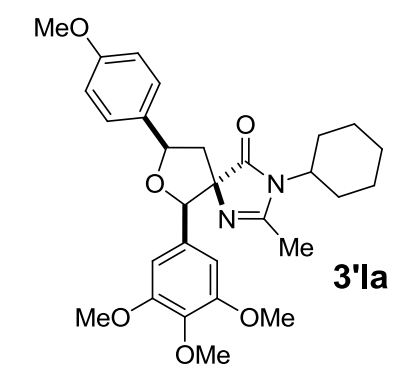

${ }^{1} \mathrm{H}$ NMR (700 MHz), $\mathrm{CDCl}_{3}$

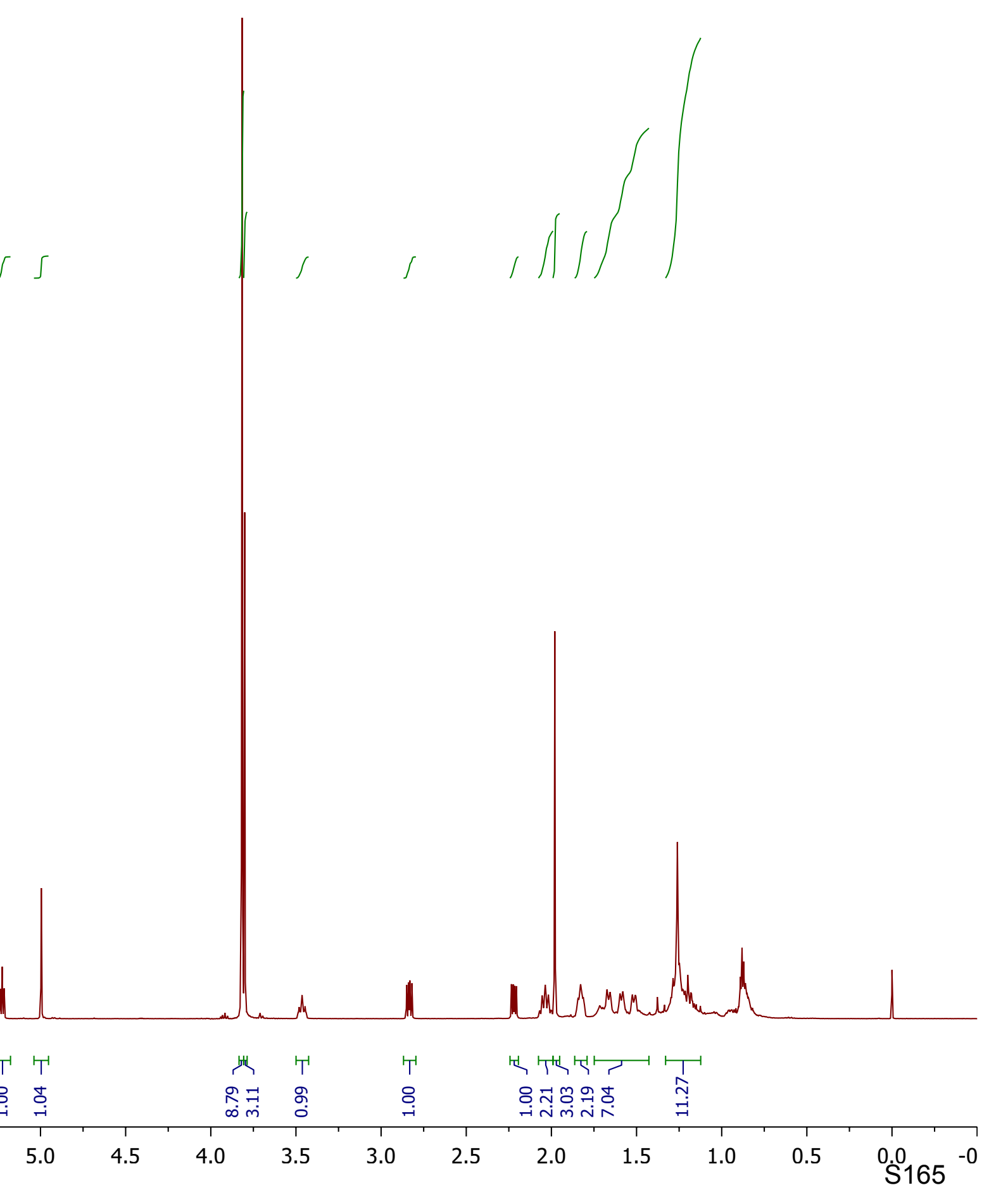




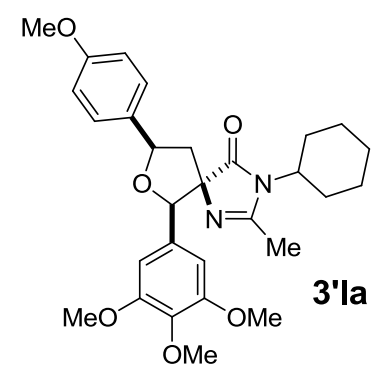

${ }^{13} \mathrm{C} \mathrm{NMR}$ (75 MHz), $\mathrm{CDCl}_{3}$

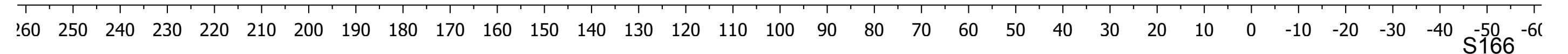




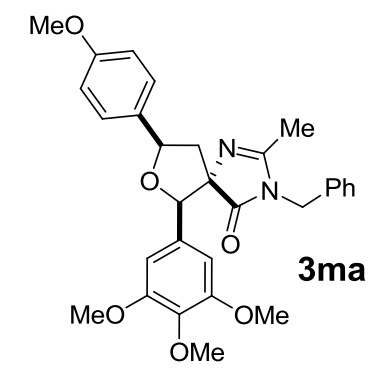

${ }^{1} \mathrm{H}$ NMR (700 MHz), $\mathrm{CDCl}_{3}$
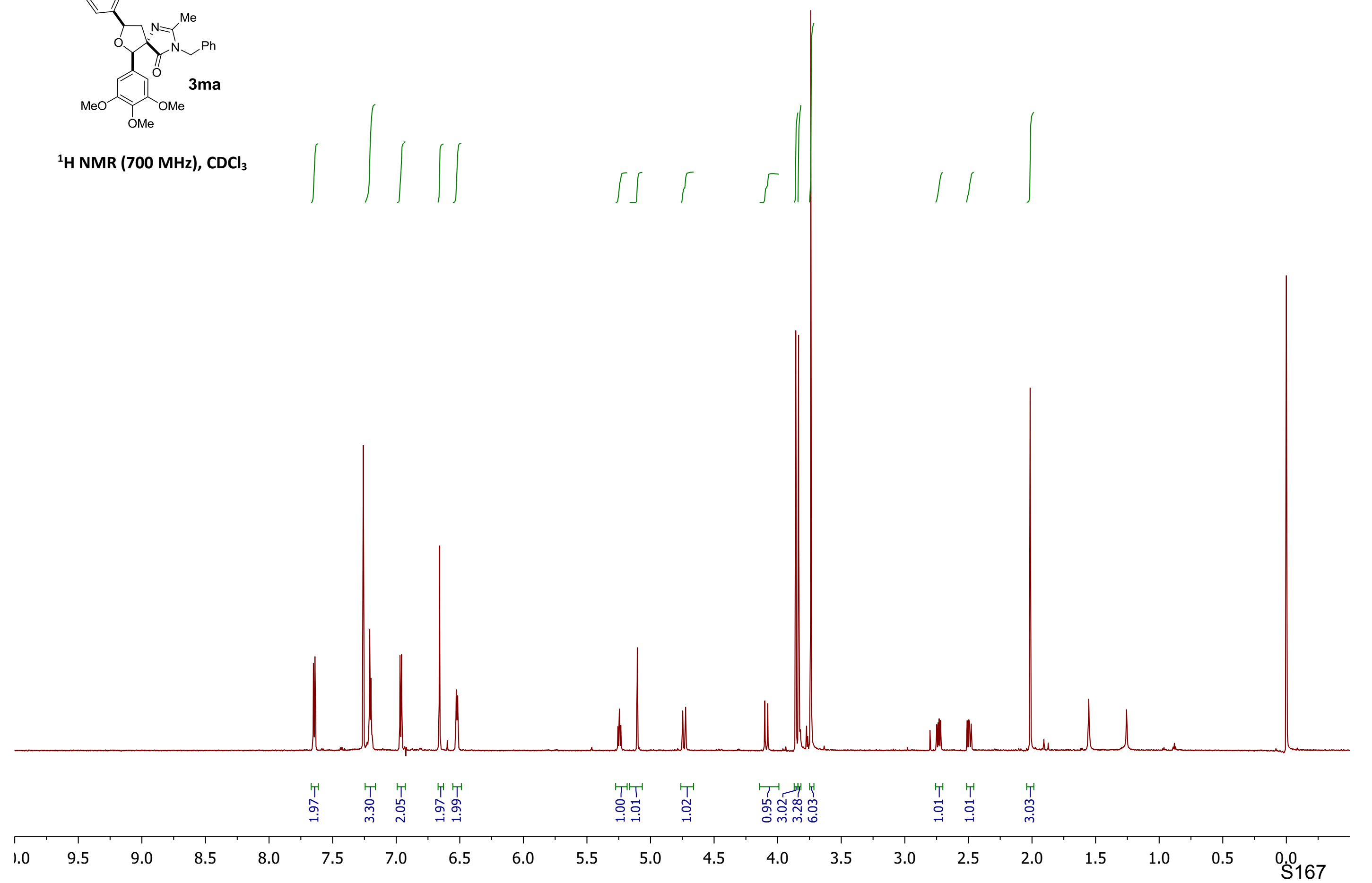
$\mathrm{MeO}$

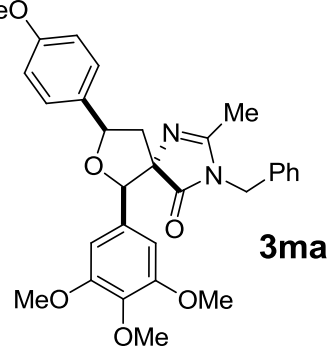

${ }^{13} \mathrm{C}$ NMR (175 MHz), $\mathrm{CDCl}_{3}$

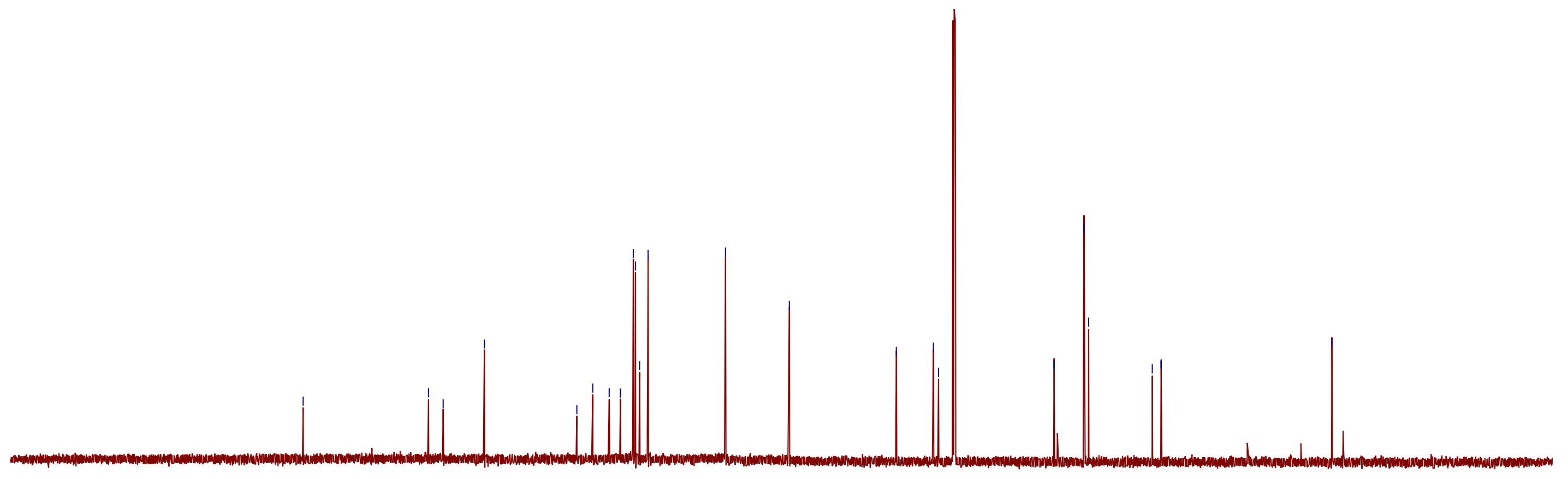




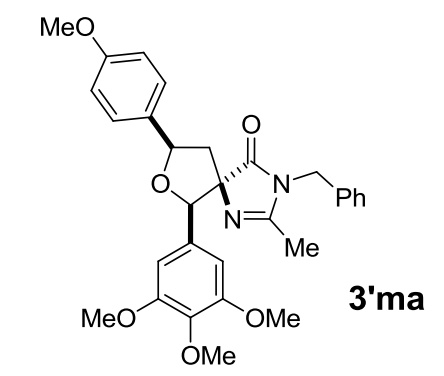

${ }^{1} \mathrm{H}$ NMR (700 MHz), $\mathrm{CDCl}_{3}$

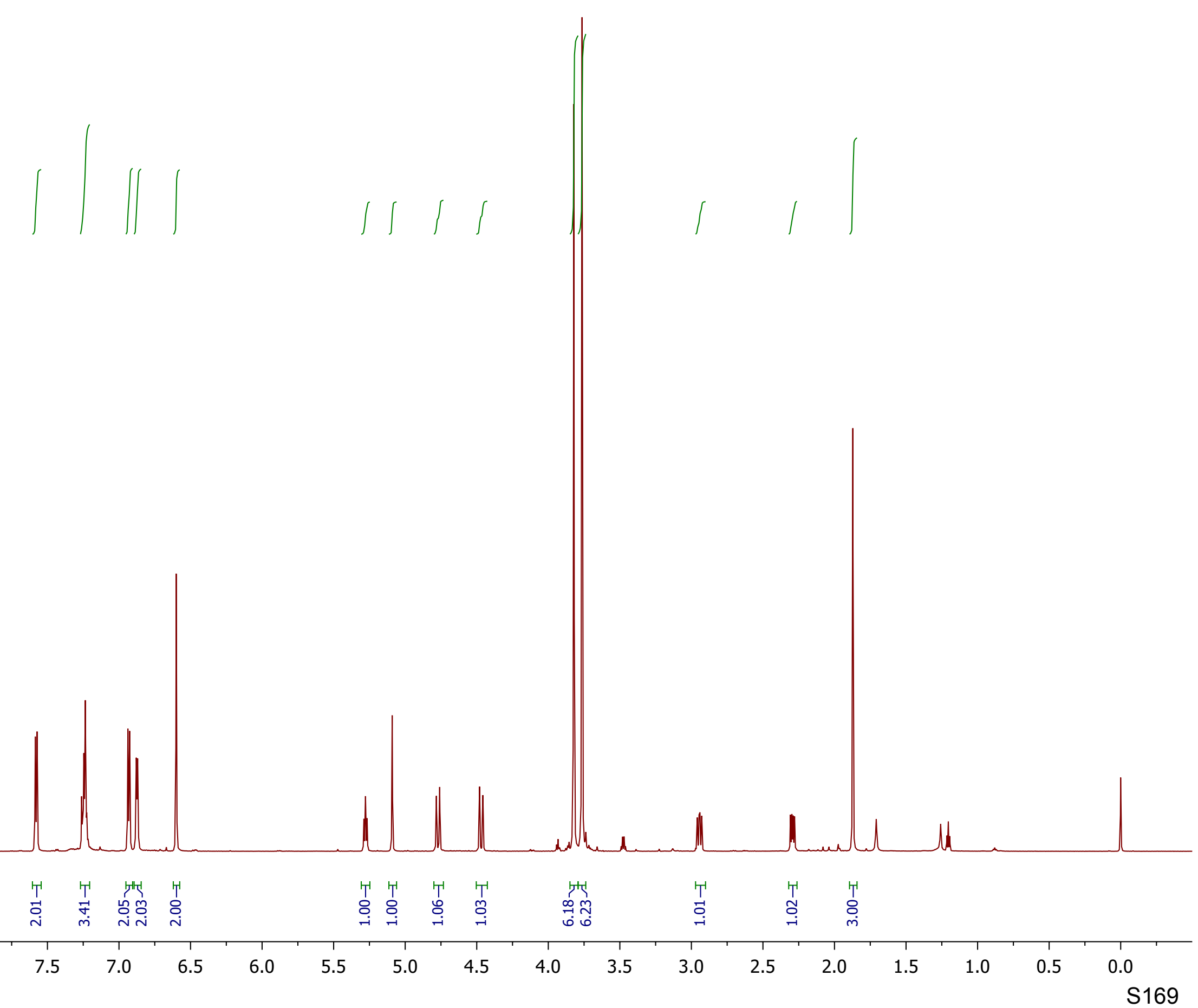




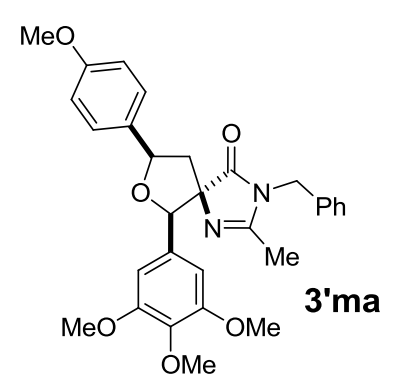

${ }^{13} \mathrm{C} \mathrm{NMR} \mathrm{(175} \mathrm{MHz),} \mathrm{CDCl}_{3}$

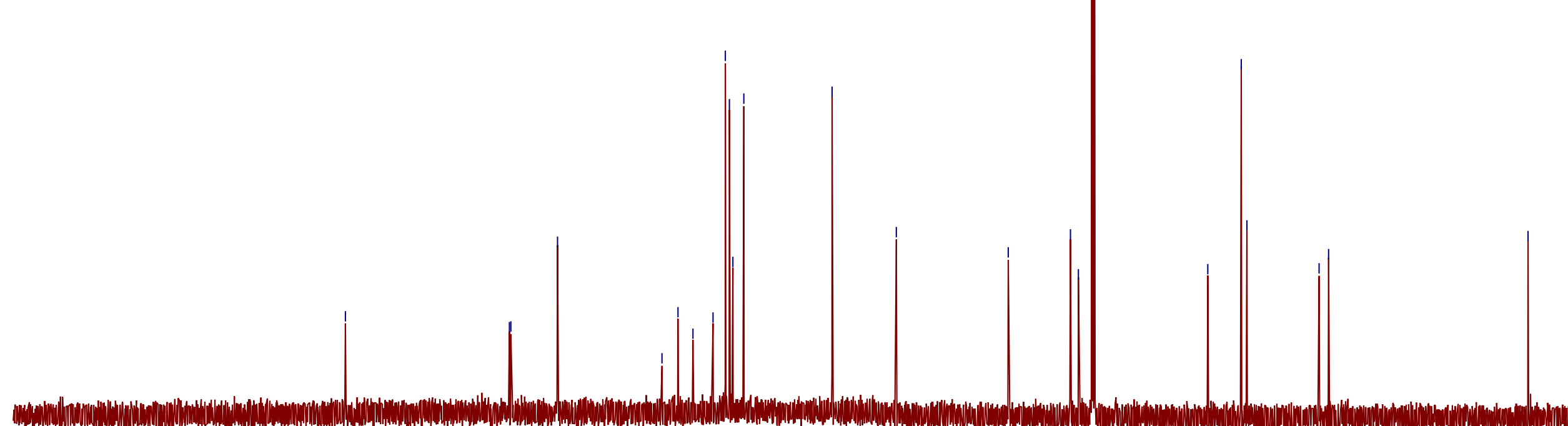




$$
\text { 3cl }
$$

${ }^{1} \mathrm{H}$ NMR (700 MHz), $\mathrm{CDCl}_{3}$

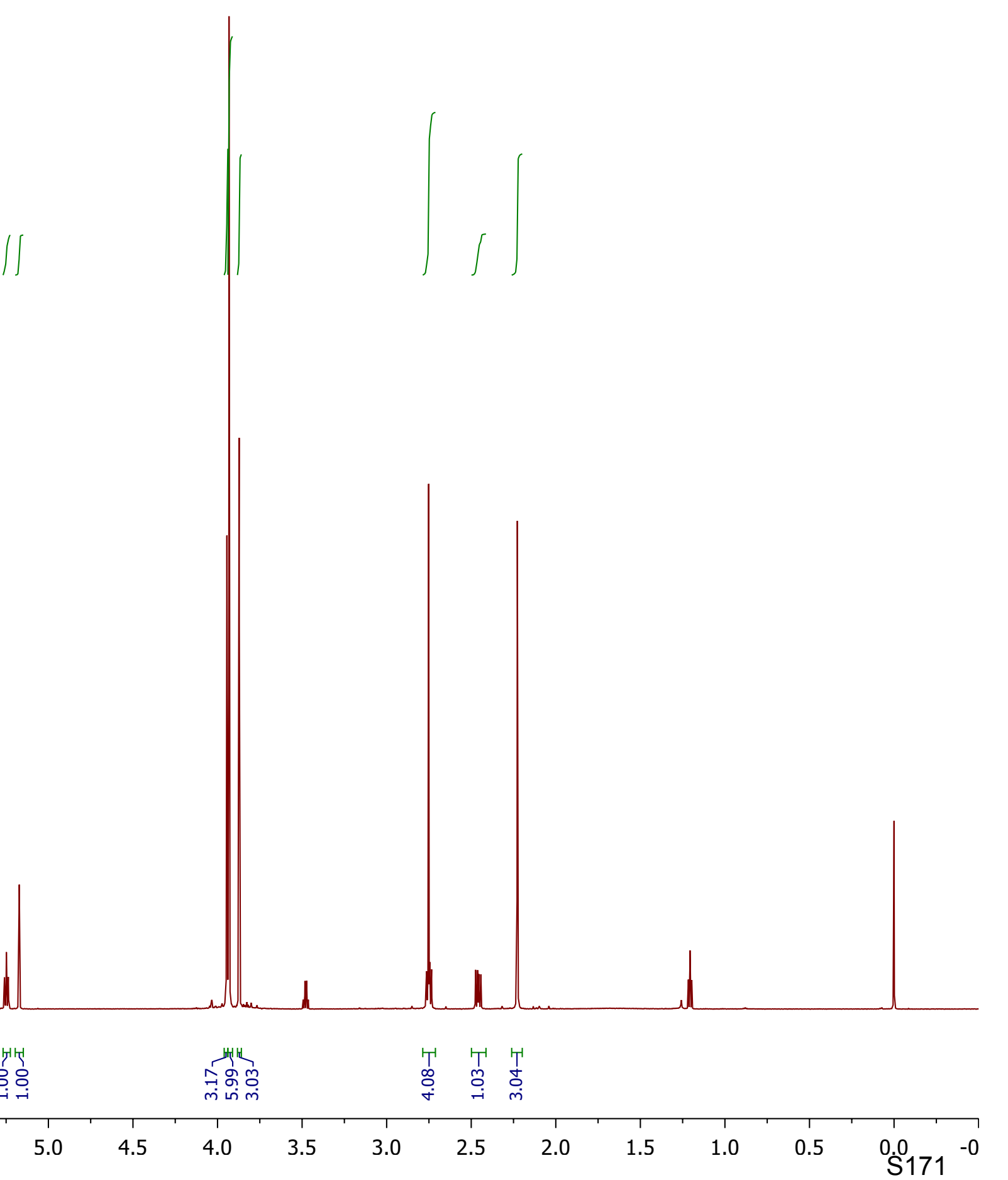





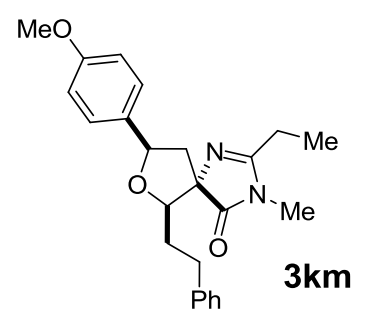

$$
{ }^{1} \mathrm{H} \text { NMR (700 MHz), } \mathrm{CDCl}_{3}
$$
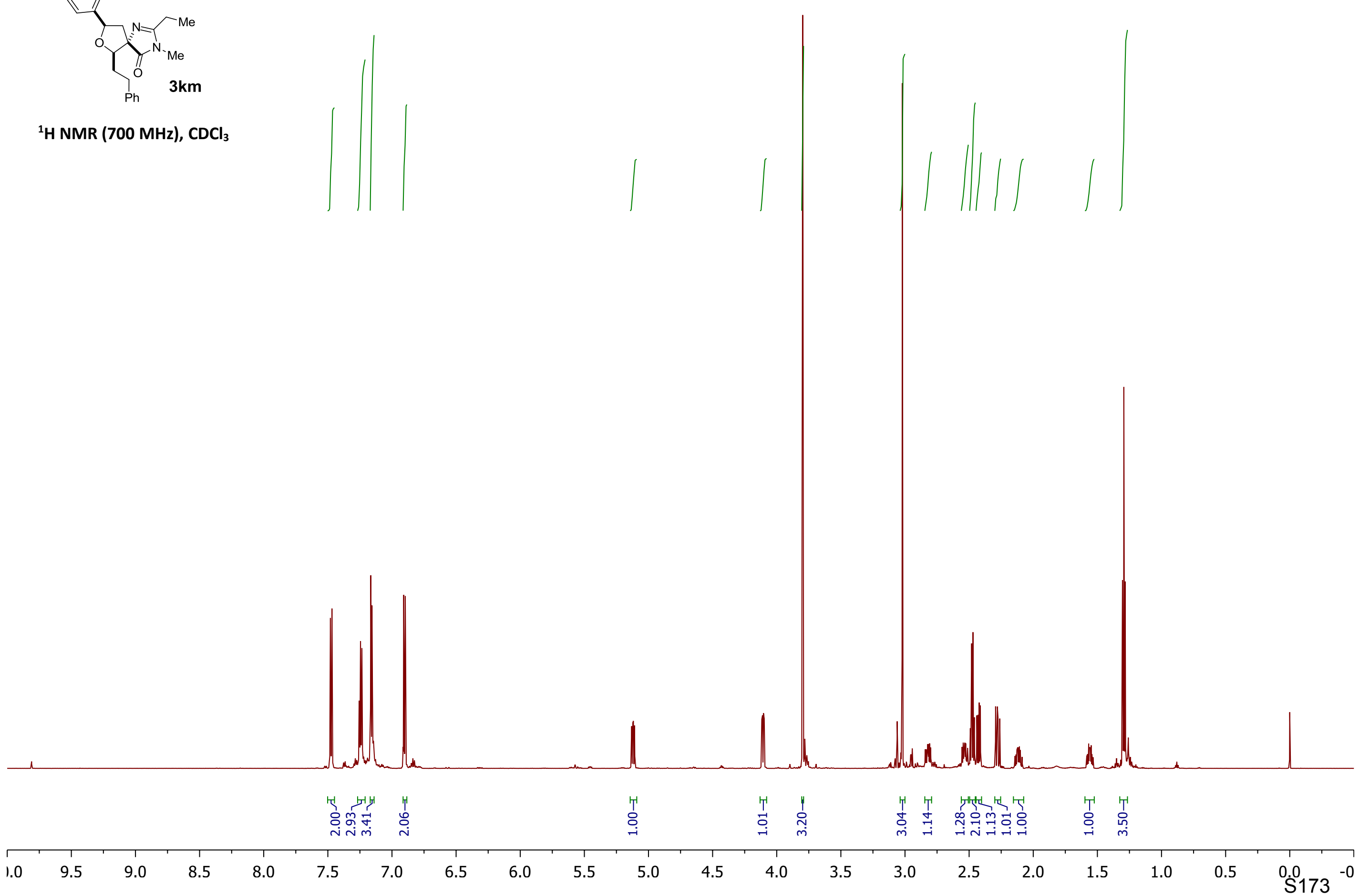


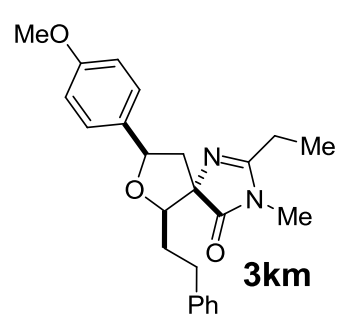

${ }^{13} \mathrm{C}$ NMR (175 MHz), $\mathrm{CDCl}_{3}$

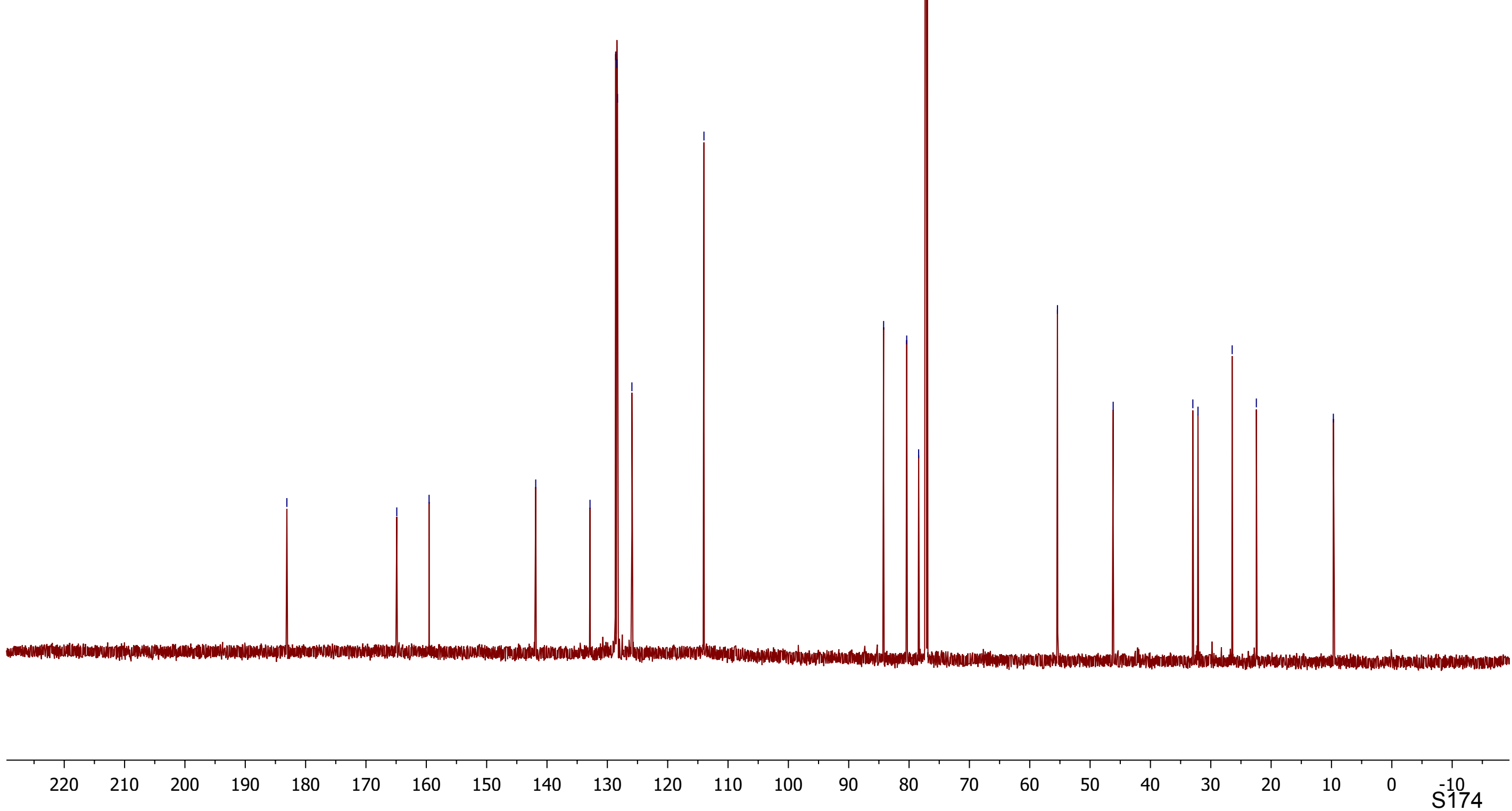




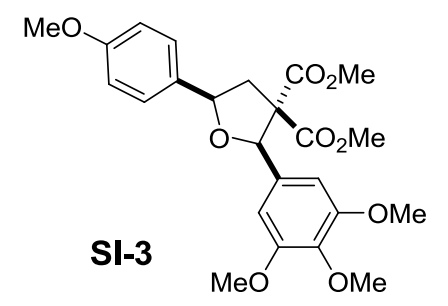

${ }^{1} \mathrm{H}$ NMR (700 MHz), $\mathrm{CDCl}_{3}$

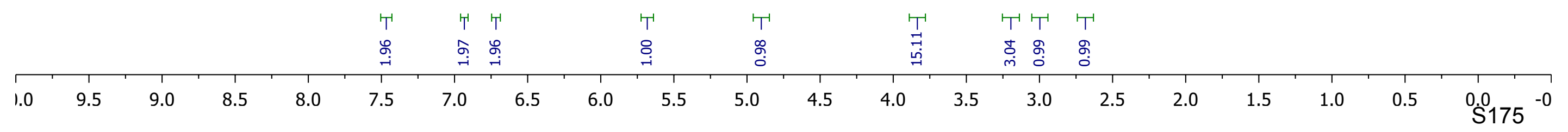




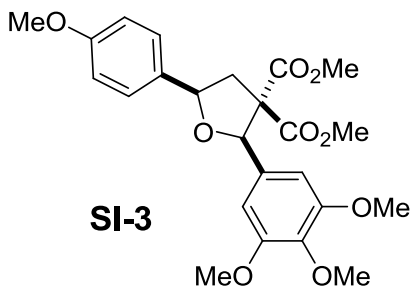

${ }^{13} \mathrm{C} \mathrm{NMR} \mathrm{(175} \mathrm{MHz),} \mathrm{CDCl}_{3}$
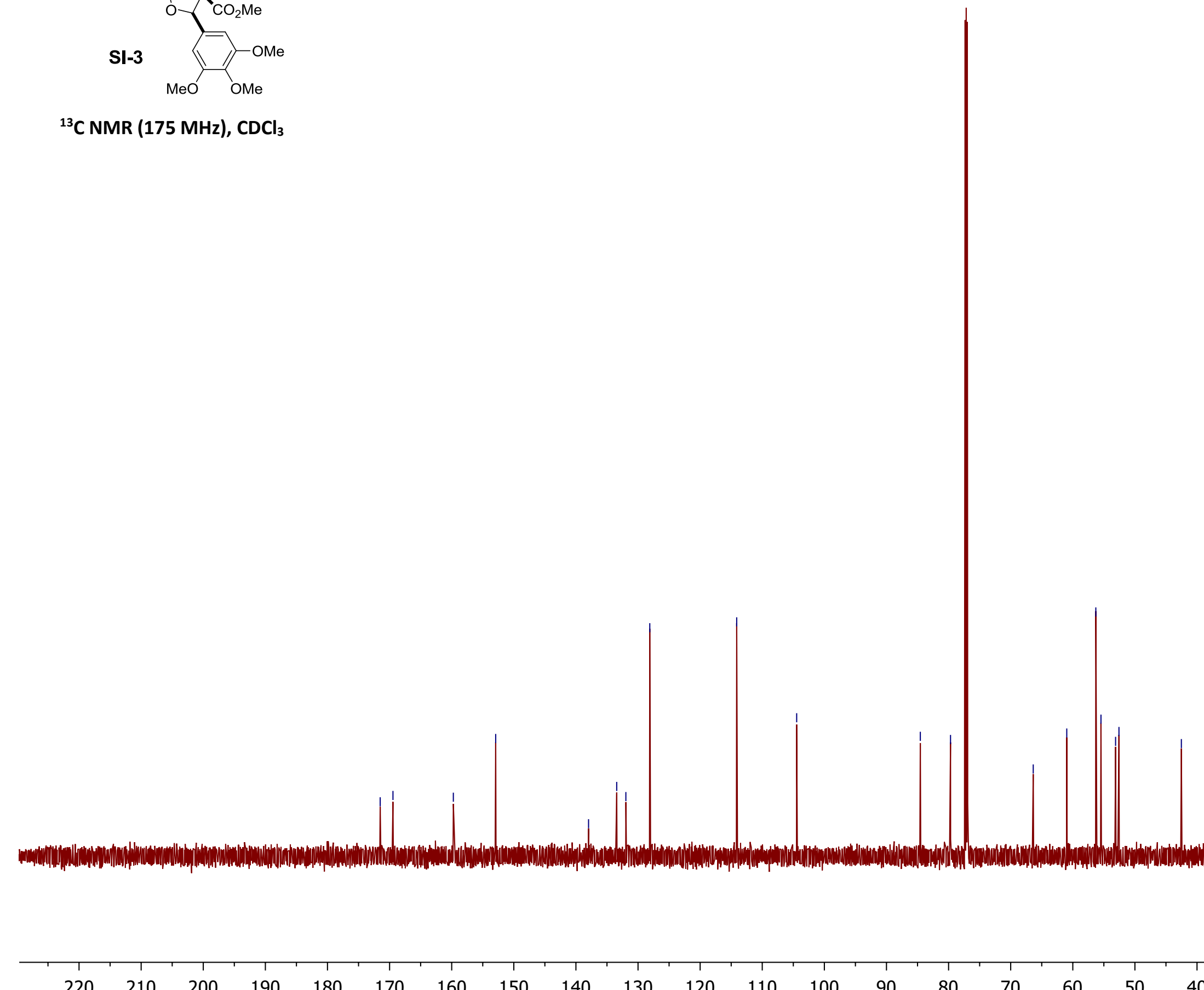


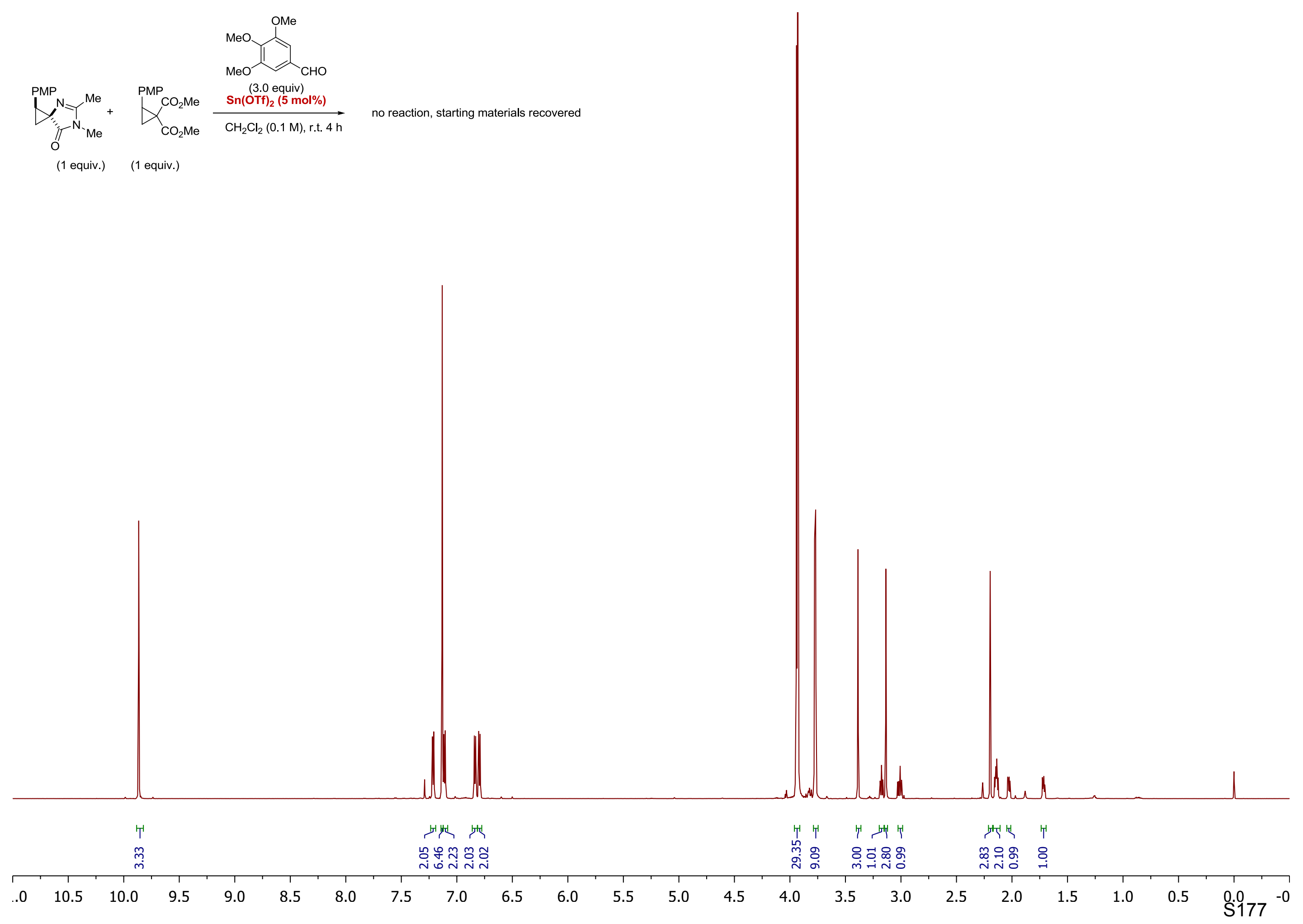




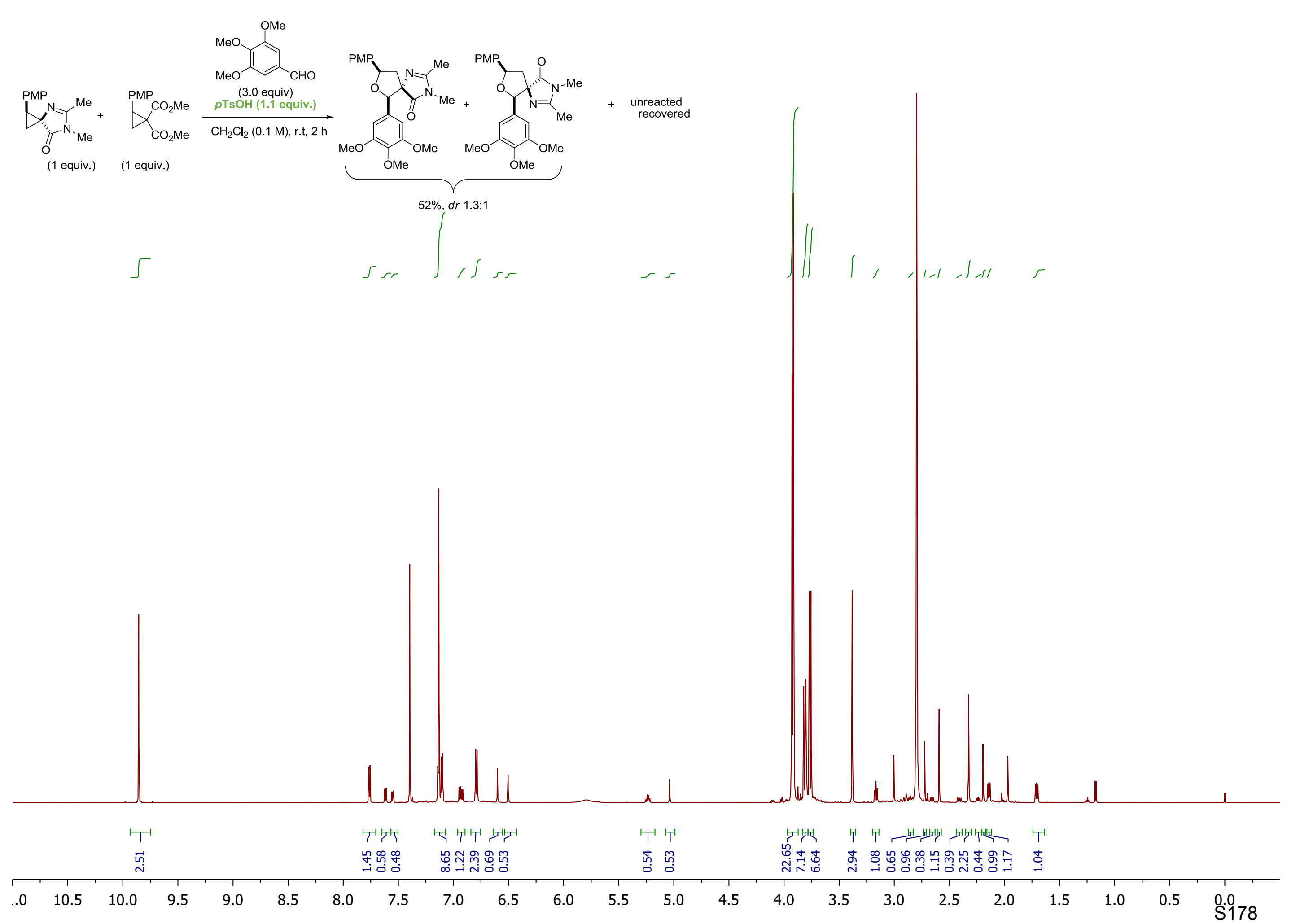

